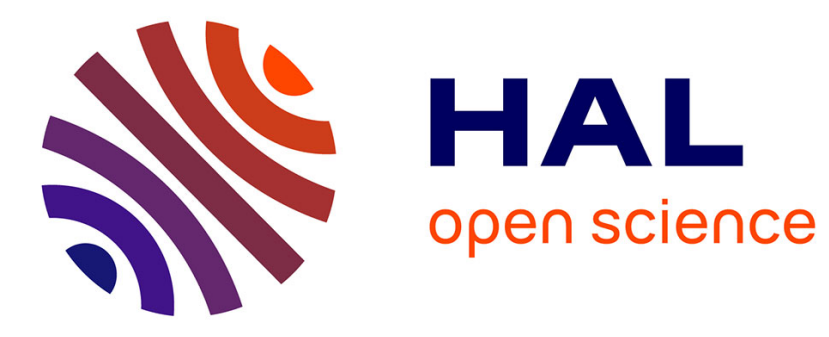

\title{
Un habitat azilien en Anjou : les Chaloignes à Mozé-sur-Louet (Maine-et-Loire)
}

Grégor Marchand, Rémy Arthuis, Sylvie Philibert, Farid Sellami, Sandra

Sicard, Philippe Forré, Sylvain Lanoë, Jean-François Nauleau, Laurent

Quesnel, Guirec Querré

\section{To cite this version:}

Grégor Marchand, Rémy Arthuis, Sylvie Philibert, Farid Sellami, Sandra Sicard, et al.. Un habitat azilien en Anjou: les Chaloignes à Mozé-sur-Louet (Maine-et-Loire). Gallia Préhistoire - Préhistoire de la France dans son contexte européen, 2009, 51, pp.1-111. 10.3406/galip.2009.2475 . hal-02022735

\section{HAL Id: hal-02022735 \\ https://hal-univ-tlse2.archives-ouvertes.fr/hal-02022735}

Submitted on 19 Dec 2019

HAL is a multi-disciplinary open access archive for the deposit and dissemination of scientific research documents, whether they are published or not. The documents may come from teaching and research institutions in France or abroad, or from public or private research centers.
L'archive ouverte pluridisciplinaire HAL, est destinée au dépôt et à la diffusion de documents scientifiques de niveau recherche, publiés ou non, émanant des établissements d'enseignement et de recherche français ou étrangers, des laboratoires publics ou privés.

\section{(1) (1) $\$$}

Distributed under a Creative Commons Attribution - NonCommercial - NoDerivatives 44.0 


\title{
UN HABITAT AZILIEN EN ANJOU: LES CHALOIGNES À MOZÉ-SUR-LOUET (MAINE-ET-LOIRE)
}

\author{
Grégor MARCHAND*, Rémy ARTHUIS**, Sylvie PHILIBERT***, \\ Farid SELLAMI**, Sandra SICARD** \\ Avec la collaboration de Philippe FORRÉ**, Sylvain LANÖ̈*, \\ Jean-François NAULEAU**, Laurent QUESNEL* et Guirec QUERRÉ*
}

Travaux dédiés à la mémoire du $\mathrm{D}^{\mathrm{r}}$ Michel Gruet

\begin{abstract}
Mots-clés. Loire, Maine-et-Loire, Azilien, Paléolithique final, habitat préhistorique, technologie lithique, galet gravé, fouille préventive. Résumé. La fouille du site des Chaloignes (Mozé-sur-Louet, Maine-et-Loire) a été réalisée en 1999, sur une surface de $9200 \mathrm{~m}^{2}$. Elle a permis pour la première fois de décrire un habitat complexe du Paléolithique final sur le Massif armoricain, tout en nous donnant une image claire des choix techniques de l'Azilien. L'environnement immédiat est fortement marqué par la Loire, avec $10 \mathrm{~km}$ de rives accessibles dans un rayon de $5 \mathrm{~km}$.

Trois thalwegs de faible importance convergent dans cette «cuvette», fermée par un étroit émissaire lié à un filon de quartz. Cette disposition naturelle a favorisé la conservation des niveaux tardiglaciaires, parfois sous un niveau d'habitat de La Tène moyenne (partie septentrionale du site), parfois sous la seule terre végétale (partie méridionale du site). Des colluvions scellent ces habitats dans tous les vallons. Les analyses géomorphologiques et micromorphologiques mettent en évidence une première phase d'érosion sous climat périglaciaire, suivie d'une phase complexe de dépôts de limons, coiffée au sommet par les vestiges aziliens. La mise en place d'un sol à ce moment désigne une amélioration climatique, que l'on corrèle à l'interstade Alleröd. Ultérieurement, ces dépôts enregistrent par endroit une reprise du froid (Dryas récent) et des troncatures sédimentaires.

Les pièces lithiques pouvant être qualifiées d'aziliennes se rencontrent au sein de dix locus homogènes, de deux zones homogènes et de deux zones à forte composante azilienne mais à intrusions postérieures. Il y a également un locus daté du Néolithique final. Les unités spatiales couvrent de $25 \mathrm{~m}^{2}$ à $70 \mathrm{~m}^{2}$. Aucun aménagement, aucun foyer, aucune zone rubéfiée, aucun effet de paroi n'ont été perceptibles. La conservation des matières organiques est compromise par l'acidité des sols du Massif armoricain. L'acquisition du silex sur les sources les plus proches a été la solution la plus couramment adoptée par les tailleurs des Chaloignes. Il s'agit pour l'essentiel de galets de terrasses de la Loire, dont les plus proches se trouvent à moins de $2 \mathrm{~km}$ de distance. Mais on observe dix matières à cortex non-roulé qui proviennent des bassins sédimentaires, en proportions variables sur les locus. L'ocre a été glanée sur les formations de grès armoricain, à une dizaine de kilomètres au nord du site, peut-être sous l'actuelle ville d'Angers (Maine-et-Loire) ou plus au nord sur la bordure du Massif armoricain. Les objectifs du débitage sont doubles: d'une part, de petits supports laminaires rectilignes à destination des armatures, d'autre part des éclats courts dont l'épaisseur est constante, pour les grattoirs. Le débitage, majoritairement bipolaire, est conduit à l'aide d'un percuteur de pierre dure, avec une faible préparation des plans de frappe. Monopointes asymétriques à dos courbe (dites pointes
\end{abstract}

\footnotetext{
* UMR 6566 du CNRS, Laboratoire d'Anthropologie, bâtiment 24-25, Université de Rennes-I, CS74205, F-35042 Rennes Cedex. Courriels : gregor. marchand@univ-rennes1.fr; laurent.quesnel@univ-rennes1.fr; guirec.querre@univ-rennes1.fr.

** Inrap Grand-Ouest, 37, rue Bignon, CS 67737, F-35577 Cesson-Sevigné Cedex. Courriels: remy.arthuis@inrap.fr; farid.sellami@inrap.fr; sandra.sicard@inrap.fr; philippe.forre@inrap.fr; jean-francois.nauleau@inrap.fr.

*** UMR 6130 du CNRS, CÉPAM, 250, rue Albert-Einstein, Sophia Antipolis, F-06560 Valbonne. Courriel: sylviep@ cepam.cnrs.fr.
} 
aziliennes), très rares lamelles à dos, burins sur troncature ou sur cassure, grattoirs unguiformes ou sur bout de lame, sont les principaux outils aménagés de l'Azilien des Chaloignes. Cette industrie offre suffisamment d'arguments pour sintégrer dans une phase récente de l'Azilien, soit la phase à monopointes, à la fin de l'interstade Alleröd (autour de $10800 \mathrm{BP}$, soit de 11000 avant notre ère).

L'ensemble des types d'outils est présent sur tous les locus; une analyse fine des proportions de pièces permet néanmoins de nuancer cette observation pour nous entraîner vers des notions de segmentation des activités dans l'espace. Une analyse fonctionnelle a permis de montrer que, hormis les activités cynégétiques dont témoignent indirectement les armatures, les processus techniques identifiés se rapportent essentiellement au travail de la peau et du bois. Une large part des produits lithiques obtenus est abandonnée sur place, sans avoir servi.

Le locus 11 est le seul à avoir livré trois galets gravés de fines stries parallèles, en position stratigraphique claire, ce qui permet sans aucun doute de les associer à l'occupation azilienne.

Le fonctionnement des locus est probablement discontinu dans le temps. Abritée des vents et disposant d'une source, la «cuvette» des Chaloignes devait offrir quelques commodités à des groupes exploitant ce versant de la Loire, avec peut-être un couvert arboré plus développé que la steppe à Poacées décrite en Mayenne par les analyses palynologiques. Le territoire économique est marqué par le fleuve, à un vaste carrefour entre plusieurs rivières.

This work is dedicated to the memory of Dr Michel Gruet

Key-words. Loire, Maine-et-Loire, Azilian, Final Palaeolithic, prehistoric habitat, lithic technology, engraved pebble, preventive excavation.

Abstract. The site of Chaloignes (Mozé-sur-Louet, Maine-et-Loire) was excavated in 1999 over surface of $9200 \mathrm{~m}^{2}$. This work led to the first description of a complex habitat of the Final Palaeolithic on the Armorican Massif and clarified our image of Azilian technical choices. The immediate environment was strongly influenced by the Loire River, with $10 \mathrm{~km}$ of banks accessible within a range of $5 \mathrm{~km}$. Three small thalwegs converge in this "basin", which is closed by a narrow outlet associated with a quartz vein. This natural deposition favoured the preservation of Tardiglacial levels, sometimes under a Middle La Tène habitat (northern part of the site), and sometimes directly under the topsoil (southern part of the site). The habitats are sealed by colluviums in all the valleys. Geomorphological and micromorphological analyses show evidence for a first phase of erosion during a periglacial climatic period, followed by a complex phase of silt deposits with Azilian remains lying on top of them. The deposition of this level at this moment attests to improvement in climatic conditions that we correlate with the Alleröd interstadial. These deposits later show elements indicating a new period of cold conditions (Late Dryas) and sedimentary truncations.

The lithic artefacts that can be attributed to the Azilian were found within ten homogeneous loci, two homogeneous zones and two others with a strong Azilian component along with posterior intrusions. There is an additional locus dated to the Final Neolithic. The spatial units cover $25 \mathrm{~m}^{2}$ to $70 \mathrm{~m}^{2}$. There is construction, hearth, rubified zone or wall effect is perceptible. The preservation of organic materials is compromised by the acidity of the sediments of the Armorican Massif. The flint knappers at Chaloignes most often acquired their raw materials from the closest sources. These materials consist essentially of cobbles from the Loire terraces, the closest of which were located less than $2 \mathrm{~km}$ away. In variable proportions in the loci, we also identified ten materials with a non-rolled cortex that originate from the sedimentary basins. Ochre was gathered from the Armorican sandstone formations, located around $10 \mathrm{~km}$ north of the site, perhaps under the current city of Angers or more to the north on the edge of the Armorican Massif. The objectives of the lithic production sequence were double: to produce small, rectilinear blade blanks to be transformed into projectile weapon elements and short flakes with a consistent thickness to be transformed into scrapers. The debitage is mostly bipolar and realized with hard stone hammer and little striking platform preparation. The principal tools in this Azilian assemblage from Chaloignes are asymmetric monopoints with an arched back (Azilian Points), a few backed bladelets, burins on a truncation or break, thumbnail scrapers and end scrapers on blades. This industry presents a sufficient number of elements to attribute it to either to a late phase of the Azilian, or to the monopoint phase at the end of the Alleröd interstadial (around 10,800 BP, or 11,000 BC).

All of the tool types are represented at all of the loci; a detailed analysis of the proportions of types nonetheless indicates a possible spatial segmentation of activities. A functional analysis showed that in addition to the hunting activities directly demonstrated by the presence of weapon elements, the technical processes identified were mostly associated with skin and bone working. Many of the lithic objects were abandoned in place without having been used.

Locus 11 is the only one that yielded three cobbles engraved with fine, parallel lines. These objects were found in a clear stratigraphic position and can thus be associated with certitude to the Azilian occupation. The functioning of the loci was probably discontinuous in time. The "basin" of Chaloignes, which was sheltered from the wind and contained a spring, must have offered certain advantages to groups exploiting this side of the Loire Valley: the forest cover was perhaps more developed here than on the step with Poaceae described by palynological analyses in the Mayenne department. The economic territory was influenced by the Loire, which composed a vast intersection between several rivers.

Translation: Magen O'FARRELL 
In memoriam Dr Michel Gruet

Schlüsselwörter. Loire, Maine-et-Loire, Azilien, Endpaläolithikum, vorgeschichtlicher Lagerplatz, lithische Industrie, Kiesel mit Ritzzeichnungen, Notgrabung.

Zusammenfassung. 1999 wurde an der Fundstelle Les Chaloignes in Mozé-sur-Louet, (Departement Maine-et-Loire) eine Fläche von $9200 \mathrm{~m}^{2}$ ergraben. Zum ersten Mal bot sich hier die Möglichkeit einen komplexen Lagerplatz des Endpaläolithikums auf dem Armorikanischen Massiv zu beschreiben und dazu ein klares Bild von den Techniken des Azilien zu vermitteln. Die unmittelbare Umgebung mit $10 \mathrm{~km}$ zugänglichen Uferzonen der Loire in einem Umkreis von $5 \mathrm{~km}$ ist stark von dem Strom gezeichnet.

Drei kleine Talwege laufen hier in einer „Mulde“ zusammen, die von einem durch eine Quarzader stark eingeengten Abzugskanal versperrt ist. Diese natürliche Beschaffenheit hat die Erhaltung der spätglazialen Schichten begünstigt, die sich teils unter einem Siedlungsniveau der Mittellatènezeit (im nördlichen Teil der Fundstätte) teils direkt unter der Muttererde (im südlichen Teil der Fundstätte) befinden. Talfüllungen versiegeln die Lagerplätze in allen Talmulden. Die geo- und mikromorphologischen Analysen zeugen von einer ersten Erosionsphase in periglazialem Klima, gefolgt von einer komplexen Phase von Schlammablagerungen, in deren obersten Schichten die Siedlungspuren des Aziliens angetroffen wurden. Die Bildung eines Belegungshorizontes zu dieser Zeit deutet auf eine Klimaverbesserung im Zusammenhang mit dem Alleröd-Interstadial. In der Folgezeit, zeichnet sich in diesen Ablagerungen stellenweise ein erneuter Temperaturrückgang (Jüngere Dryas) ab, und die Sedimente weisen einen durch die Erosion hervorgerufenen Bruch auf. Die dem Azilien zugeordneten Steinartefakte fanden sich an zehn homogenen Stellen mit besonders hoher Fundkonzentration, an zwei homogenen Zonen, und an zwei weiteren Zonen, in denen das Azilien zwar vorherrschte, die jedoch jüngere Einschlüsse aufwiesen. Darüber hinaus wurde eine Fundstelle in das Endneolithikum datiert. Die Zonen umfassten 25 bis $70 \mathrm{~m}^{2}$. Es waren weder Spuren irgendeiner räumlichen Organisation, einer Feuerstelle, eines geröteten Bereichs, noch irgendein Wandeffekt erkennbar. Die Erhaltung der organischen Materialien wird durch den hohen Säuregehalt der Böden des Armorikanischen Massivs beeinträchtigt. Den Feuerstein besorgte man sich in den Chaloignes üblicherweise aus den nächstliegenden Lagerstätten. Es handelte sich im Wesentlichen um Kiesel der kaum 2 km entfernten Flussterrassen der Loire, doch daneben wurden in unterschiedlichen Proportionen zehn weitere Arten von Feuersteinknollen aus den sedimentären Becken beobachtet. Der Ocker wurde in den Schichten armorikanischen Sandsteins gesammelt, die nur etwa $10 \mathrm{~km}$ weiter nördlich, vielleicht unter der modernen Stadt Angers lagern oder noch weiter nördlich am Rand des Armorikanischen Massivs. Mit dem Abbau wurden zwei Ziele verfolgt: einerseits wurden kleine geradlinige Klingengrundformen für die Mikrolithen produziert, andererseits kurze Abschläge mit konstanter Dicke für die Kratzer. Der zumeist bipolare Abbau erfolgte mit einem Schlagstein bei einer groben Vorbereitung der Abschlagflächen. Asymmetrische Einzelspitzen (sog. Azilienspitzen), sehr seltene Rückenmesser, Stichel auf Endretusche oder Bruch, daumennagelförmige Kratzer oder Klingenkratzer sind die wichtigsten Artefakte des Azilien in den Chaloignes. Dieses Inventar bietet genug Argumente, um in eine jüngere Phase des Azilien, am Ende des AllerödInterstadials (um 10800 B.P. bzw. 11000 v. Chr.) eingeordnet werden zu können.

An allen Fundstellen war das gesamte Gerätespektrum vertreten. Um sich ein genaueres Bild von der räumlichen Aufteilung der Aktivitäten zu machen, konnten diese Beobachtung durch eine Feinanalyse der Proportionen der einzelnen Fundkategorien jedoch noch nuanciert werden. Eine funktionelle Analyse hat gezeigt, dass ausgenommen die Jagd, von der die Pfeilspitzen indirekt zeugen, die identifizierten technischen Prozesse im Wesentlichen mit der Verarbeitung von Fellen und Holz in Zusammenhang standen. Ein großer Teil der Steinartefakte wurde unbenutzt vor Ort an der Produktionsstelle liegengelassen.

Die Fundstelle Nr. 11 ist die einzige, die in eindeutiger stratigraphischer Position drei Kiesel mit feinen parallelen, eingeritzten Rillen geliefert hat, wodurch sie ohne jeden Zweifel dem Azilien zugeordnet werden kann.

Die einzelnen Plätze wurden wahrscheinlich diskontinuierlich besucht. Die windgeschützte und über eine Quelle verfügende „Mulde“ der Chaloignes bot den Gruppen, die diesen Abschnitt der Loire nutzten, wahrscheinlich gewisse Bequemlichkeiten; zudem dürfte die Bewaldung dichter gewesen sein als in der Steppe in Poacées, die in der benachbarten Mayenne durch Pollenanalysen nachgewiesen wurde. Das wirtschaftliche Territorium war von dem Strom an der Einmündung mehrerer Flussläufe bestimmt.

Übersetzung: Isa ODENHARDT-DONVEZ

\section{CARACTÈRES GÉNÉRAUX DE L'INTERVENTION}

La fouille d'un vaste site azilien de plein air prend une dimension particulière dans le contexte de la recherche préhistorique française actuelle. Si les travaux des années 1970-1980 dans le Bassin aquitain et le seuil du Poitou, puis des années 1990 dans le Bassin parisien, ont nettement fait progresser notre connaissance de la fin du Tardiglaciaire, le Massif armoricain était resté rigoureusement hors de ce renouvellement des données. Quelques caractères techniques des industries humaines ont certes été fixés en Pays-de-la-Loire par M. Allard (Allard, 1983; Allard, Gruet, 1976) et en Bretagne par J.-L. Monnier (Monnier, 


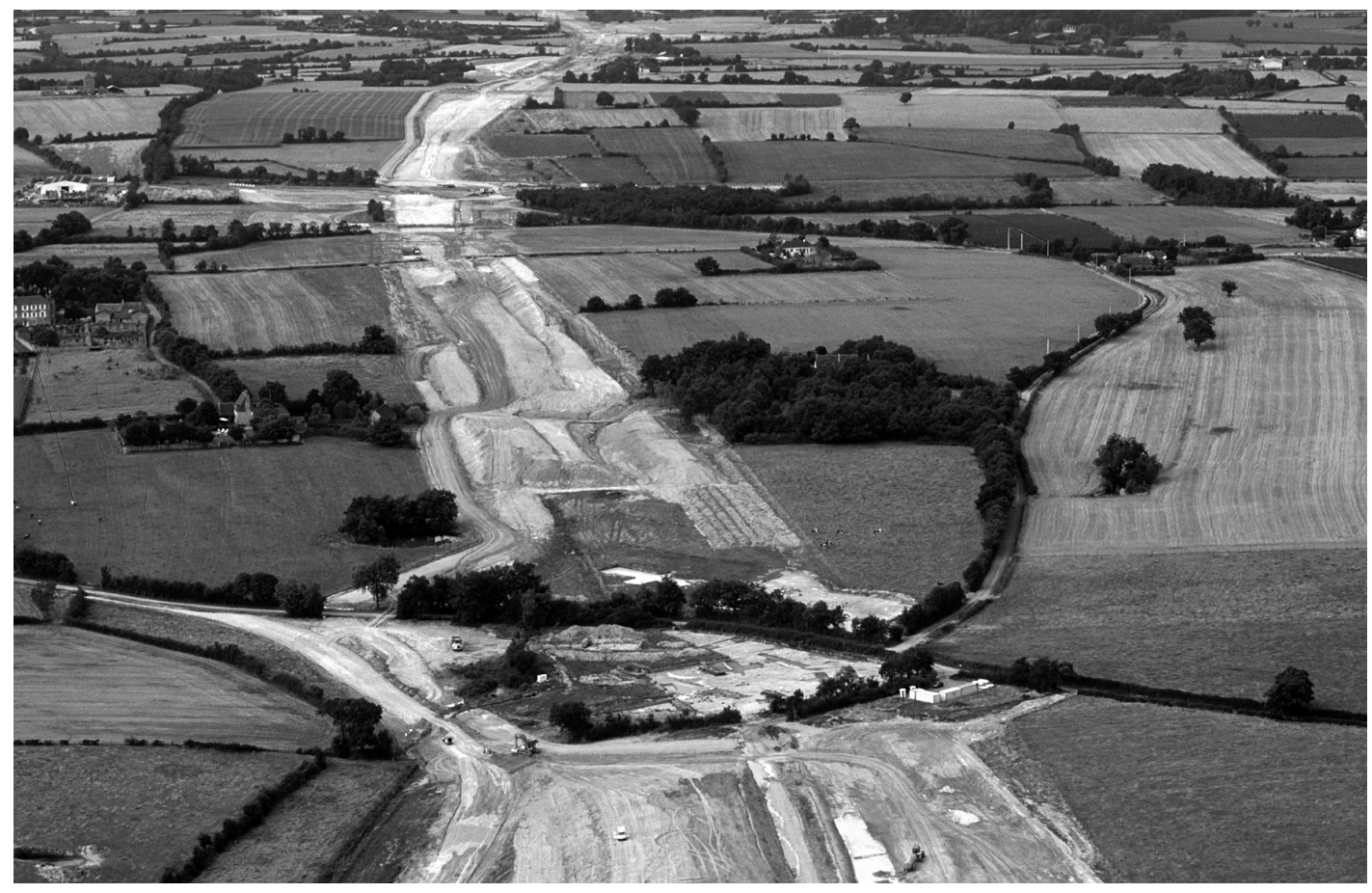

Fig. 1 - Vue aérienne du site des Chaloignes, sur le tracé de l’A87 (vue du nord, cliché: H. Paitier, Inrap).

1980), mais aucun site n'offrait suffisamment d'ampleur et d'homogénéité pour apporter des informations scientifiques comparables à celles des régions voisines. Les travaux de prospections de surface en Bretagne (Gouletquer et al., 1996), en Pays-de-la-Loire (Gouraud et al., 1998) ou en Poitou-Charentes (Foucher, San Juan, 1994), faisaient bien apparaître sporadiquement des pointes aziliennes. Elles venaient seulement signaler une occupation humaine, qui par ailleurs ne faisait pas de doute tant elle était manifeste dans les régions limitrophes. En parallèle, les diagrammes polliniques réalisés par L. Visset et D. Barbier permettaient de décrire pour la première fois à l'est du Massif armoricain des environnements contemporains de la fin de la glaciation (Barbier, 1999; Barbier, Visset, 2000). Les premières pointes aziliennes exhumées en août 1998 aux Chaloignes arrivaient à point nommé pour ouvrir un nouveau chapitre de la Préhistoire de l'Ouest.

L'un des enjeux des études sur les Chaloignes est de fournir un référentiel des caractères typologiques et technologiques de l'Azilien de l'Ouest, dès lors que les accumulations de silex de dimensions restreintes attestent de séjours "courts", mesurés bien sûr à l'aune de l'échelle de temps accessible sur le Massif armoricain. L'autre enjeu est de comprendre ce lieu dans le réseau de peuplement qui lui donne un sens. Après les habitats aziliens, les hommes du
Mésolithique fréquentent sporadiquement cette zone basse. Une occupation du Néolithique final dans l'interfluve sud-ouest est davantage perceptible par les pièces lithiques abandonnées. Plusieurs bâtiments et des structures afférentes (foyers, fosses, fossés) ont été érigés lors du second âge du Fer (La Tène moyenne; Marchand et al., 2000a; Levillayer, 2006). Un ensemble de fosses creusées au milieu du vallon ouest atteste de préoccupations peut-être symboliques, puisqu'un thorax humain côtoyait des ossements d'animaux, des tessons, des bracelets en lignite et des perles en verre. Le paysage est ensuite marqué par des aménagements agricoles usuels, visant à lutter contre l'engorgement des vallons. La cuvette des Chaloignes devient donc épisodiquement un nœud important dans une organisation géographique particulière et nous tenterons d'en comprendre les raisons pour la fin du Paléolithique.

Parce qu'elle se trouvait au beau milieu du tracé de l'autoroute A87 (Angers/La Roche-sur-Yon), la cuvette des Chaloignes, au nord-ouest de la commune de Mozé-surLouet, devait faire l'objet d'une intervention de sauvetage ${ }^{1}$ (fig. 1). Elle s'est déroulée d'avril à septembre 1999, sur

1. Position du site en coordonnées Lambert zone II étendue: $\mathrm{X}=381.200 ; \mathrm{Y}=2268.300$. Responsable de l'opération: $\mathrm{G}$. Marchand; responsables de secteur: S. Rimbaud et S. Sicard. 
une surface totale de $9200 \mathrm{~m}^{2}$. Dans la zone centrale de la cuvette (versant nord du vallon ouest), où un niveau protohistorique surmontait immédiatement le niveau préhistorique, le décapage a été particulièrement délicat. Le temps imparti à l'opération ne permettait pas de réaliser deux fouilles successives. Pour la première partie des opérations, l'ablation de la terre végétale et des limons récents s'est faite à l'aide d'une pelle mécanique. La fouille sur les locus aziliens a été manuelle, avec le recours au tamisage soit total, soit par échantillon.

G. M.

\section{LE CONTEXTE SÉDIMENTAIRE}

\section{CADRE GÉNÉRAL ET LOCALISATION DU SITE}

La route nationale 160, en venant de Beaulieu-surLayon (Maine-et-Loire), offre une vue d'ensemble sur les paysages de la vallée de la Loire, avec pour ligne d'horizon, le profil accidenté de la ville d'Angers (fig. 2 et 3). Dans cet espace, l'effet de pente et la vue dégagée attirent inexorablement vers le fleuve. C'est ici, sur la rive gauche de la Loire, proche de la rivière de l'Aubance, à l'abri des vents au sein de la petite vallée du ruisseau de la Fontaine de Mont, que furent découverts les vestiges d'une occupation humaine du Tardiglaciaire. Dans cette zone située à une centaine de kilomètres de l'Atlantique, la Loire, lien naturel et dynamique entre l'océan et le continent, a répercuté, via la rivière de l'Aubance, les effets sédimentaires de la transgression flandrienne jusque dans la basse vallée du ruisseau de la Fontaine de Mont (prélèvement inédit de Lionel Visset). Ainsi, au gré des fluctuations océaniques qui se mesurent à l'échelle des temps géologiques, la Loire et ses affluents ont parfois remblayé, mais plus efficacement entaillé la frange méridionale du Massif armoricain. Cette dynamique d'ensemble explique la forme étroite, profonde et en forte pente de la moyenne vallée du ruisseau de la Fontaine de Mont. En revanche, la haute vallée se présente sous forme d'un vaste amphithéâtre aux pentes douces, drainée par trois vallons faiblement ramifiés. C'est au sein de ce replat légèrement dépressionnaire que le site est conservé. Ces caractéristiques morpho-topographiques ont favorisé une stagnation temporaire d'une grande partie des eaux de surface collectées par les trois vallons faiblement encaissés dans des versants à pente douce. Nous avons distingué les vallons ouest, sud et est, mais aussi les interfluves correspondant nommés sud-ouest, sud-est et nord-est (fig. 4).
Les interfluves latéraux, sud-est et nord-est, sont relativement moins larges que l'interfluve frontal sud-ouest (fig. 5). En effet, les deux premiers se rejoignent sur le site par des flancs courts en pente faible et régulière alors que le second, frontal, se développe sur un flanc plus long. La rupture de sa pente est située bien en amont du site ce qui permet des déplacements sédimentaires longs et bien triés. Ces variations morpho-topographiques ont engendré des dynamiques sédimentaires spécifiques à chaque zone. Le croisement des différents vallons a fait de ce replat une zone temporairement mouillée permettant le développement d'une végétation de prairie humide. Cette zone forme la source du ruisseau de la Fontaine du Mont qui a permis l'évacuation des eaux et, en partie, des sédiments vers la vallée, régulant ainsi le degré d'humidité et le rythme de la sédimentation au sein du site. Ceci explique la présence de formations superficielles relativement peu épaisses notamment sur les pentes où le substrat remonte presque en surface.

Les vestiges archéologiques aziliens s'étendent autour des trois vallons et dans la cuvette. Ceux de l'âge du Fer sont essentiellement représentés par une zone d'habitat sur le versant nord. Des vestiges du Mésolithique et du Néolithique final, plus ténus, ont été découverts dans la cuvette et dans l'interfluve sud-ouest. De nombreux indices archéologiques indiquent que l'ensemble de l'espace a été occupé de manière sporadique durant la période protohistorique, antique, médiévale, moderne et contemporaine.

\section{CONTEXTE GÉOLOGIQUE}

D'après la carte géologique (Cavet et al., feuille d'Angers XV), le substrat géologique est constitué de formations d'âges différents: Paléozoïque, Secondaire, Tertiaire et Quaternaire. Dans les environs proches du site, ces formations sont essentiellement des schistes à grès, à calcaire et à quartz d'âge paléozoïque. Ces schistes, intégrant d'importants filons de quartz, affleurent en abondance dans les environs proches du site. L'altération assez avancée de la majorité de ces formations schisteuses a facilité leur désagrégation et donc leur transfert d'un endroit à l'autre selon la pente de la surface du sol. Ainsi leurs transferts latéraux par colluvionnement et par ruissellement ont significativement contribué à la formation des dépôts quaternaires. Leur accumulation sur le site des Chaloignes a été favorisée par trois facteurs morphologiques stationnels: la rupture de la pente au niveau du site, la régularité de la 


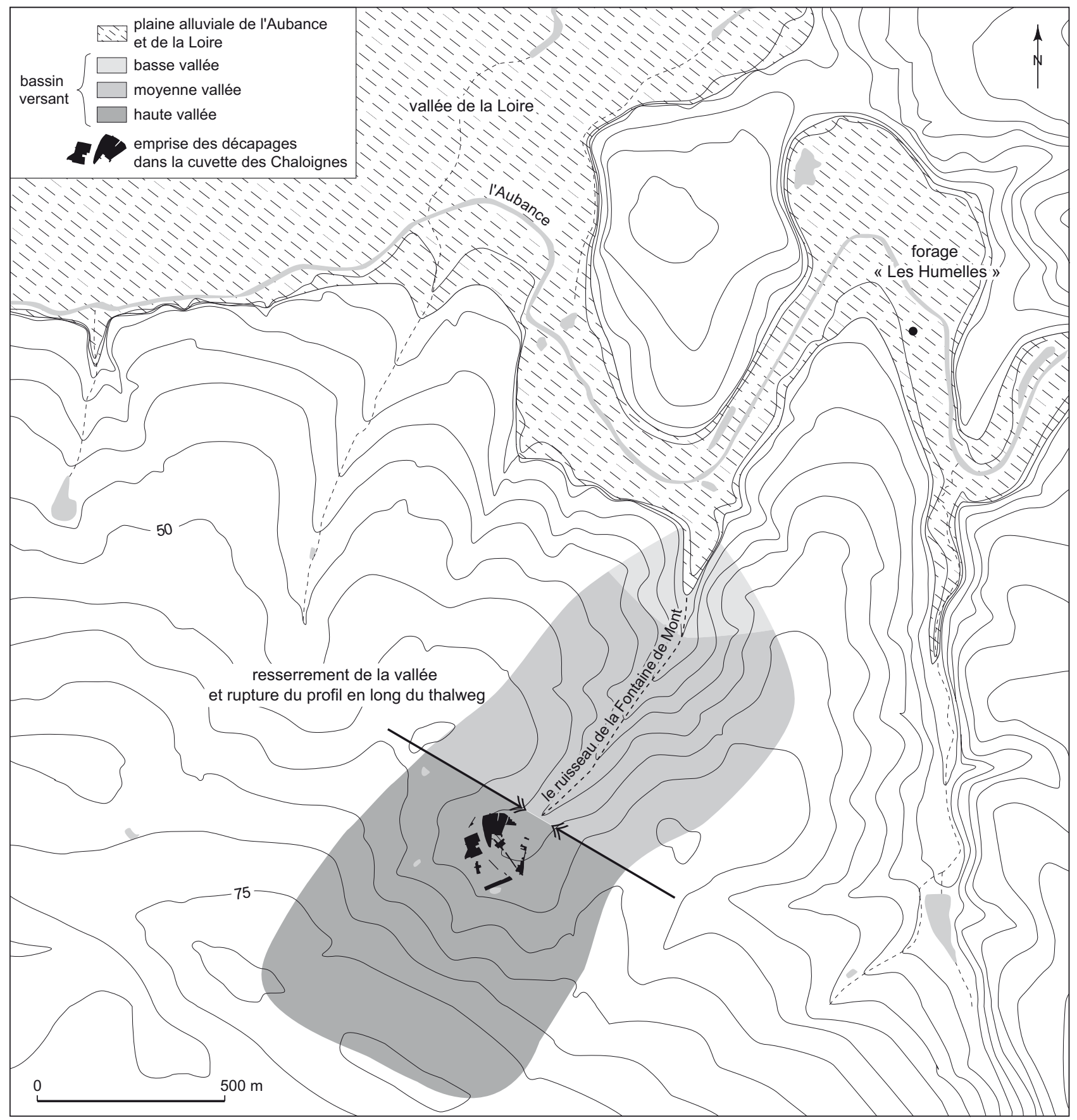

Fig. 2 - La vallée du ruisseau de la Fontaine de Mont et le site des Chaloignes; en noir, les surfaces décapées (dessins: J.-F. Nauleau, Inrap).

surface du sol et la canalisation des dynamiques hydriques dans les vallons. La combinaison de ces facteurs a permis une stabilisation temporaire de la surface du sol et une sédimentation rapide, indispensable à la conservation des sols et des niveaux archéologiques.
Nos observations de terrain ont montré la présence au fond de la cuvette de galets épars et de lentilles sableuses provenant vraisemblablement de l'érosion des blocs de poudingues observés en position secondaire, en amont du bassin versant. La présence de ces poudingues ne surprend 


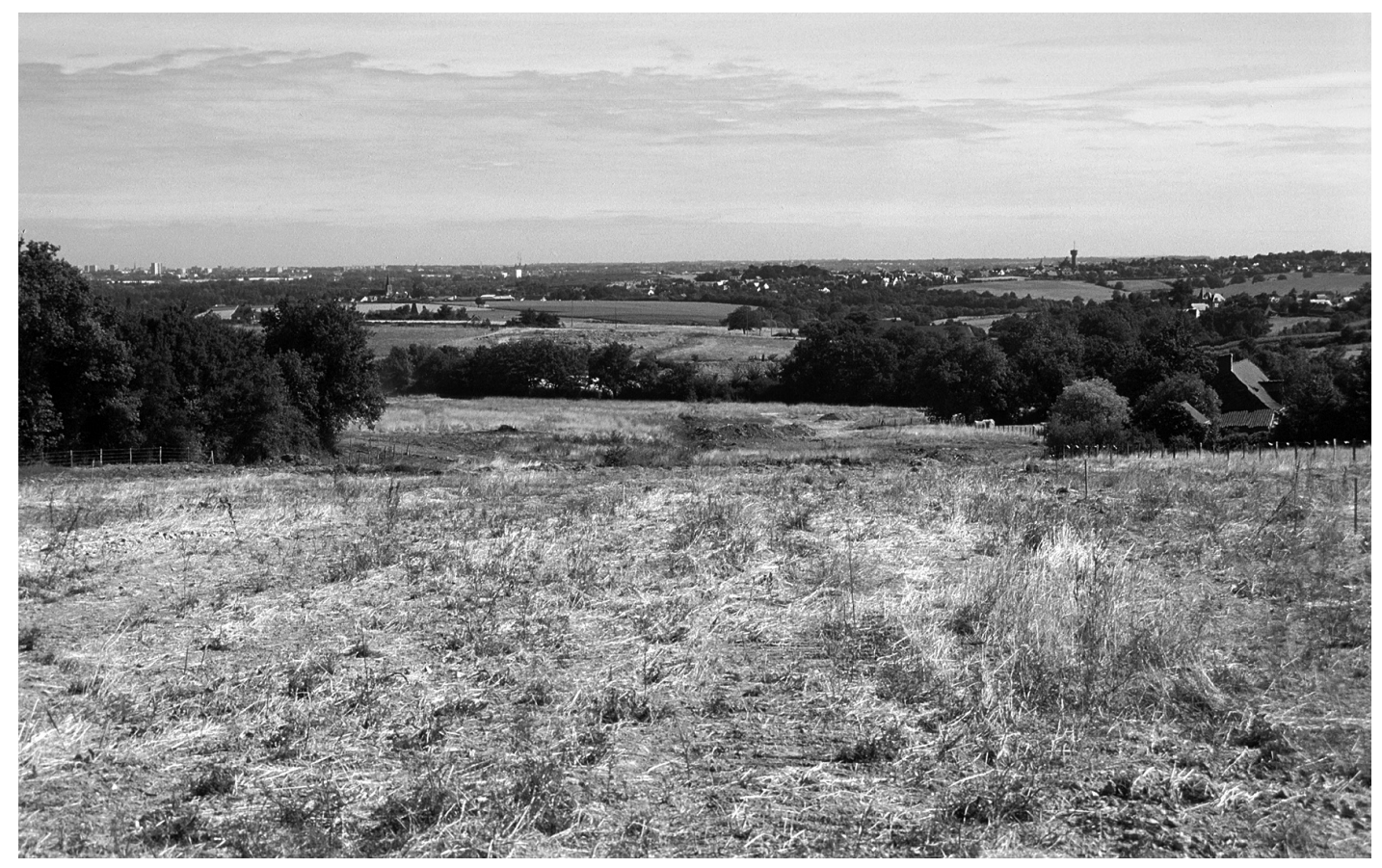

Fig. 3 - Vue générale de la cuvette des Chaloignes, dans l'axe du vallon sud. Au fond à gauche, la ville d’Angers (cliché: G. Marchand, CNRS UMR 6566).

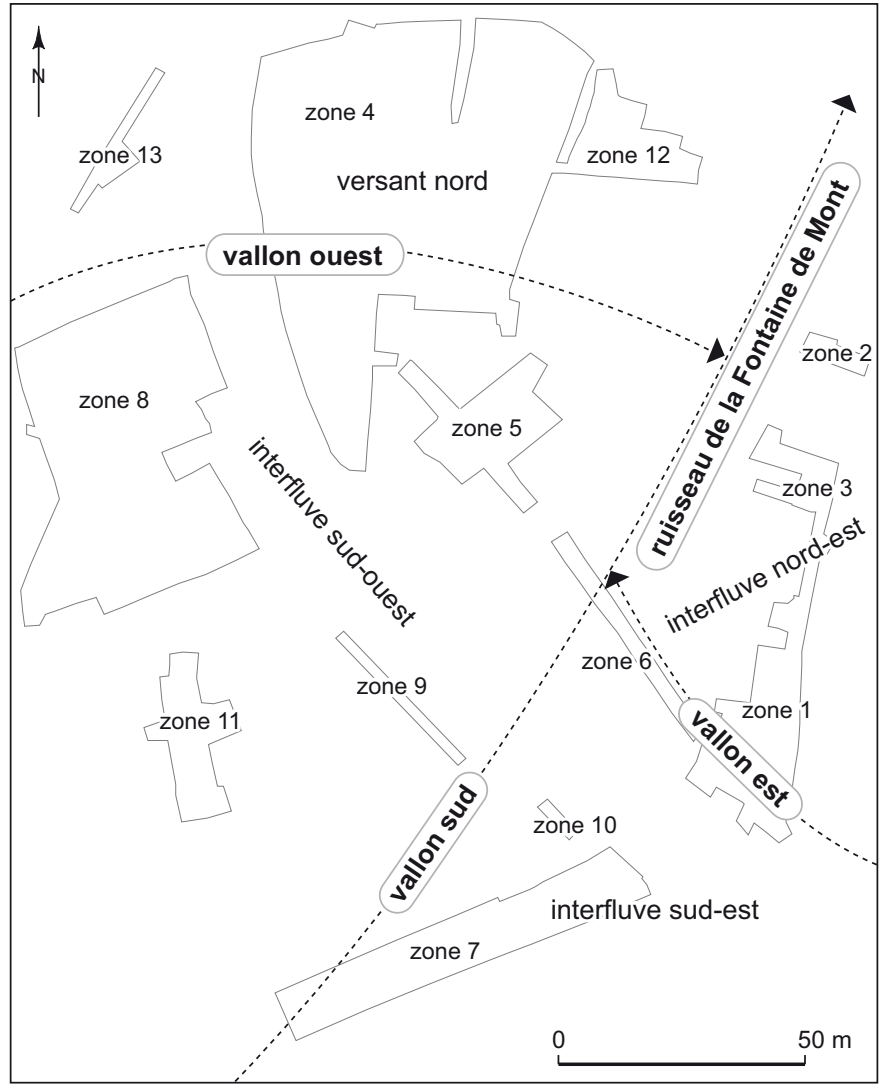

Fig. 4 - Nomenclature adoptée pour la description des unités topographiques et des zones de fouille de la cuvette des Chaloignes (dessin: G. Marchand, CNRS UMR 6566). 

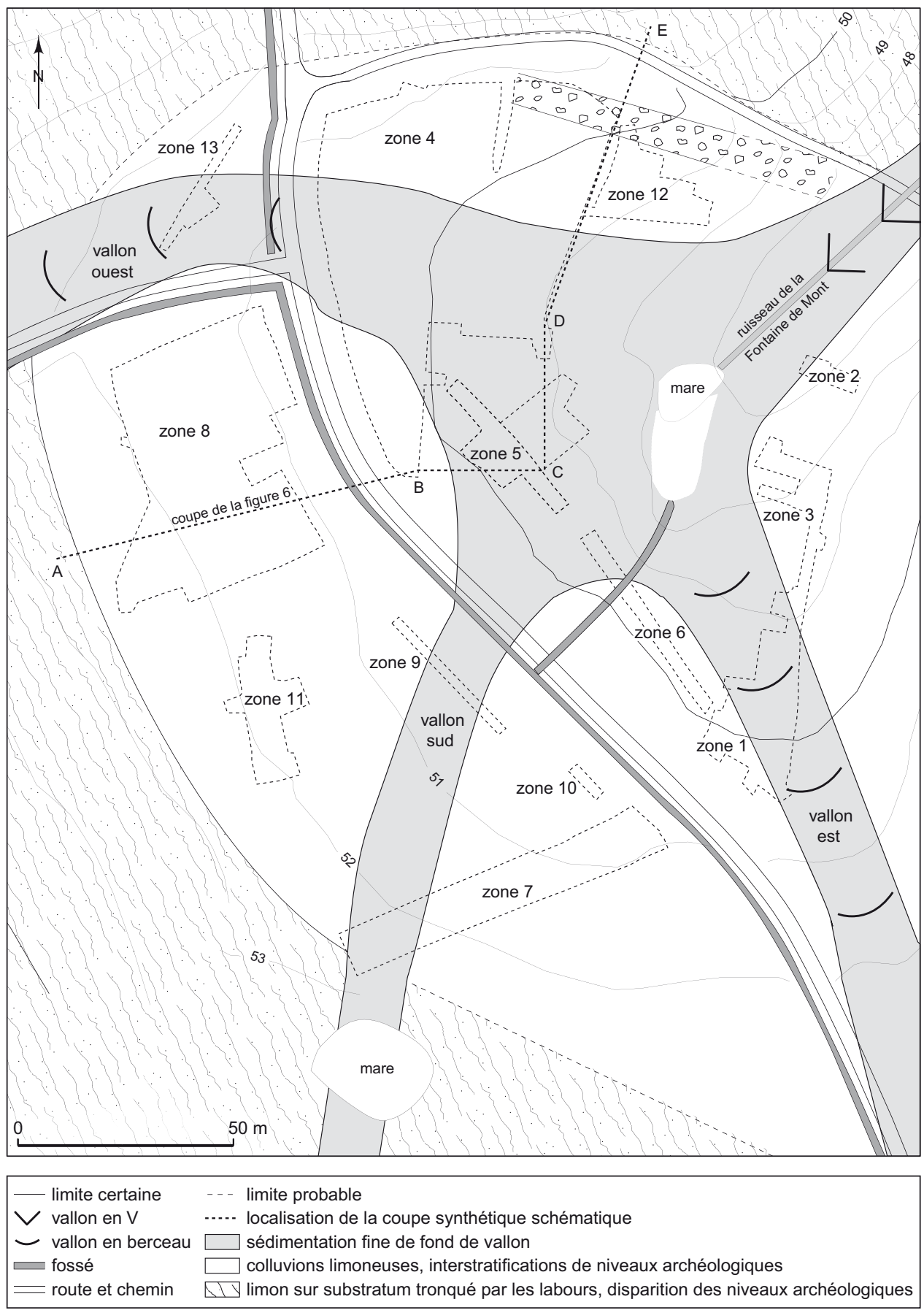

Fig. 5 - Représentation des sédiments de surface et position de la coupe synthétique de la figure 6 (conception: R. Arthuis, Inrap; dessins: J.-F. Nauleau, Inrap).

aucunement si l'on considère le nombre et la variété des formations alluviales répertoriées sur la carte géologique. Ils traduisent l'importance des érosions fluviatiles et marines dans ce secteur au cours des périodes pléistocènes et ante-quaternaires.
Parmi ces formations alluviales, citons, pour exemple, les terrasses anciennes de l'Aubance et de la Loire, les formations marines, comme celles du Crétacé, présentes au sud du Layon, ou encore les dépôts marins pliocènes fortement redistribués. 


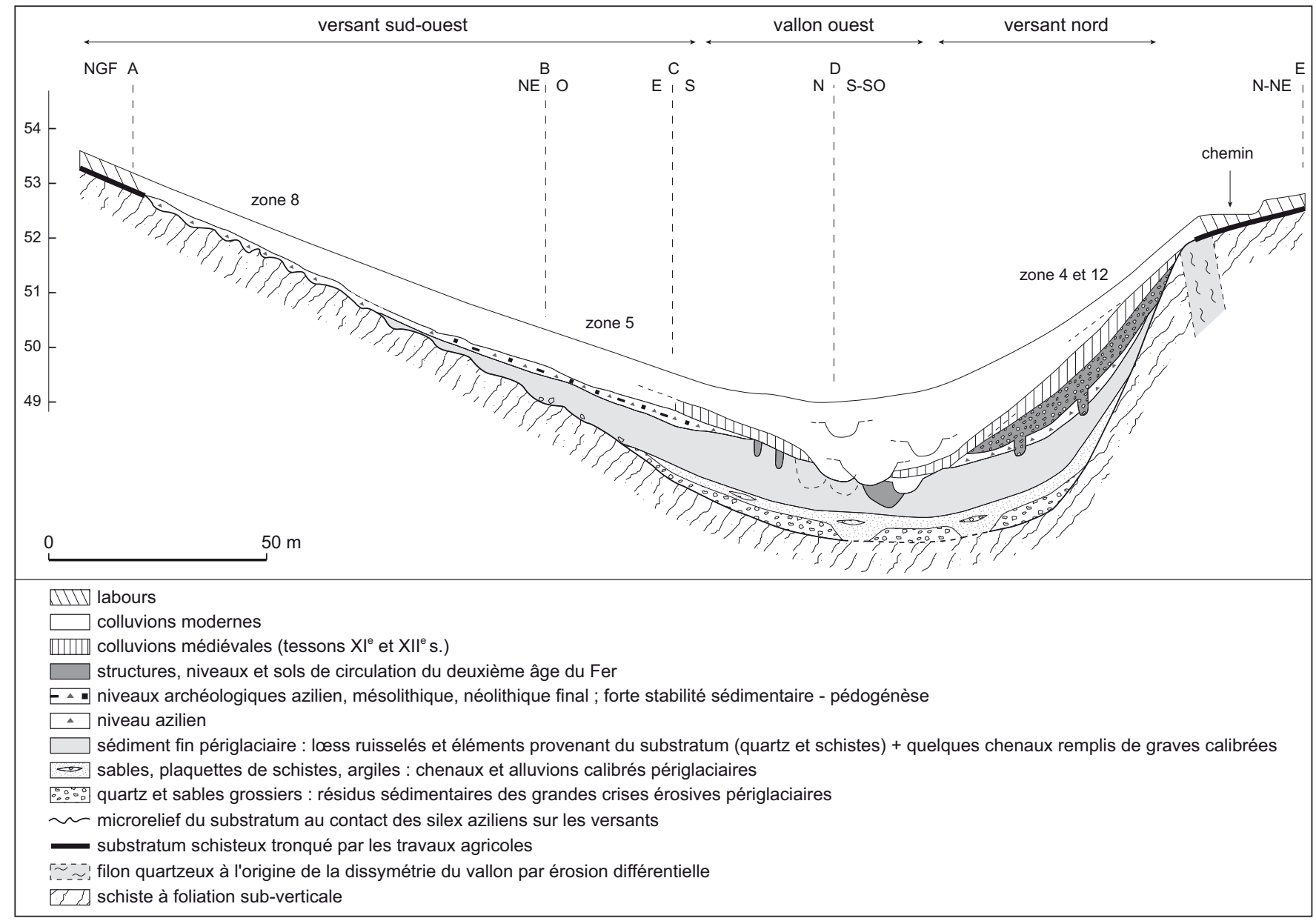

Fig. 6 - Coupe géologique synthétique (conception: R. Arthuis, Inrap; dessins: J.-F. Nauleau, Inrap).

\section{LA GÉOMÉTRIE DU SUBSTRAT ET SON IMPACT SUR LA RÉPARTITION DES DÉPÔTS SÉDIMENTAIRES}

L'activité des processus érosifs en amont du site a entraîné un transfert régulier des sédiments et un décapage progressif de la partie superficielle des schistes fragilisée par l'altération. Ces décapages ont été à l'origine de la mise au jour du substrat en amont du site, ce qui s'est traduit par une nette diminution des formations pédologiques limitées alors à celles du sol actuel (fig. 6). La canalisation de la dynamique hydrique au sein des vallons bien marqués sur les pentes a, en revanche, incisé ce substrat dont la surface est devenue alors très irrégulière. Cette incision apparaît importante dans la cuvette des Chaloignes: malgré la multiplication des sondages, le substrat n'a pas été atteint sous l'effet de la remontée rapide de la nappe. Il résulte de ces processus érosifs une ondulation du substrat géologique qui a nettement influencé la répartition spatiale de la couver- ture sédimentaire. En effet, si les versants ne sont recouverts que par une vingtaine de centimètres de sédiments, les bas de pente ont accumulé une bonne épaisseur de matériaux, notamment dans la zone dépressionnaire du site. Cette accumulation a permis le rétablissement du profil topographique, ce qui a nettement diminué l'importance de la dynamique hydrique et des transferts sédimentaires.

\section{CONTEXTE STRATIGRAPHIQUE ET ÉCHANTILLONNAGE}

La complexité géomorphologique a nettement influé sur la conservation et l'organisation des dépôts sédimentaires sur le site. Leur matérialisation a été discontinue, ce qui a requis la réalisation de nombreuses coupes stratigraphiques dans les différentes zones du site permettant l'exposition de la totalité des niveaux sédimentaires. La majorité de ces coupes a montré des matériaux globalement comparables, malgré les nuances de texture, de développement 

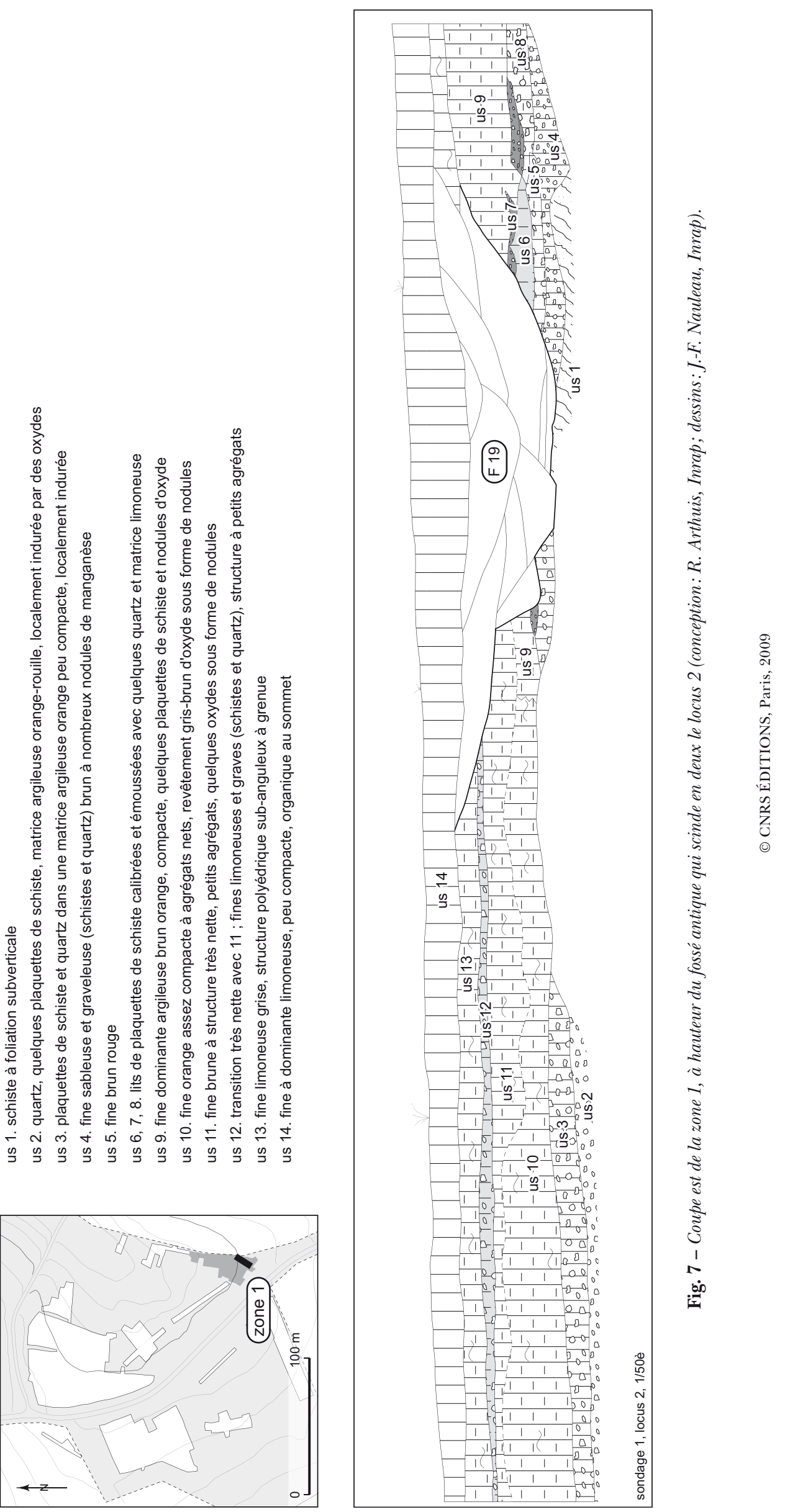


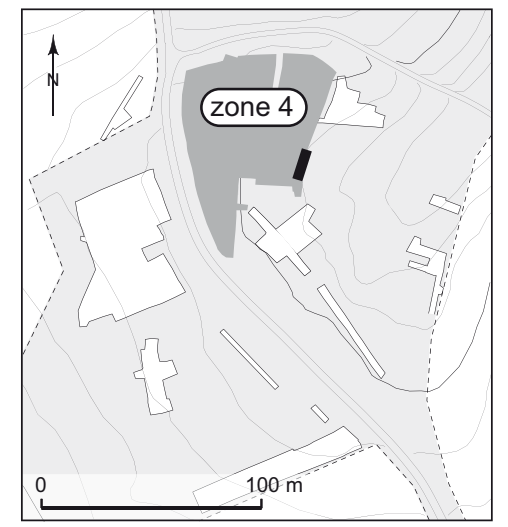

us $1,2,3$. limon argileux orange clair compact avec quelques quartz us 4,5 . limon argileux orange avec oxydes de manganèse et fer us 6 . limon, fragments de schiste roulés, quartz émoussés niveau azilien us 7. limon argileux orange niveau âge du Fer us 8 . fine graveleuse orange us 9,10 . limon, quelques plaquettes de schiste us 11,12 . limon brun

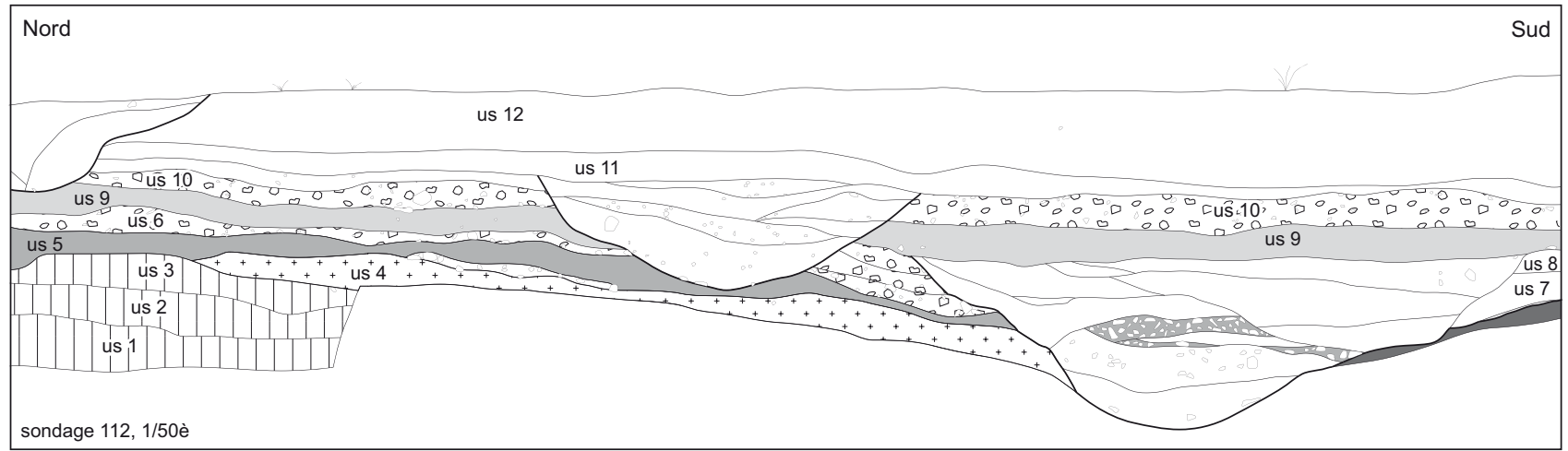

Fig. 8 - Détail de la coupe est de la zone 4 (conception: R. Arthuis, Inrap; dessins:J.-F. Nauleau, Inrap).

pédologique et d'organisation sédimentaire répondant à des fonctionnements de facteurs stationnels spécifiques à chaque zone. Nous présentons ici la description de trois coupes principales sélectionnées au sein des zones 1, 4 et 5. Ces zones représentent le milieu de dépôt des matériaux provenant des versants dominant le site. En effet, les accumulations sédimentaires y sont relativement importantes et complexes et les niveaux archéologiques y sont mieux représentés, notamment au sein de la zone 1 et 5 . Nous tenterons d'en décrire du bas vers le haut les formations sédimentaires qui seront la base de la discussion générale sur la dynamique des formations sédimentaires et leur évolution post-dépositionnelle.

Dans la zone 1, les formations sédimentaires sont latéralement continues en dehors des creusements anthropiques matérialisés par des fosses et des fossés de dimensions variables. Leur organisation verticale, leur texture et leur structure sédimentaire ont permis de distinguer 14 unités stratigraphiques (fig. 7). Au sein de la zone 4, les formations sédimentaires ne diffèrent pas sensiblement de celles de la zone 1 (fig. 8). Elles sont composées dans leur grande majorité de limons argileux. Les variations du degré de développement pédologique ont permis de différencier 12 unités stratigraphiques. La description de la zone 5 a permis de matérialiser trois niveaux sédimentaires principaux formés par des matériaux très similaires à ceux évoqués dans les zones 1 et 4 (fig. 9).

La ressemblance des matériaux parentaux au sein des différentes zones n'a pas diminué la complexité stratigraphique des coupes notamment lors du fonctionnement simultané des processus pédo-sédimentaires naturels et anthropiques. C'est le cas dans les zones 1 et 5 qui ont fait l'objet d'échantillonnage en vue d'une analyse micromorphologique afin de:

- définir la nature et l'origine des apports sédimentaires,

- corréler les formations sédimentaires entre ces deux zones 1 et 5 ,

- évaluer le degré de remaniement post-dépositionnel du niveau azilien et distinguer l'impact des processus naturels de celui des activités anthropiques dans l'évolution des sols.

Les prélèvements collectés au sein de cette zone 1 proviennent des couches 10, 11, 12 et 13, soit dans et autour des 


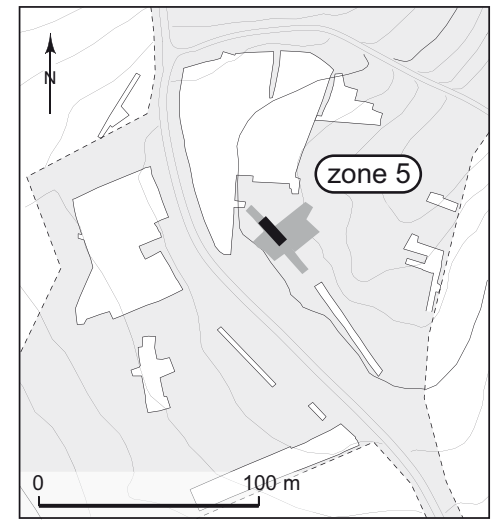

us 1, 2. limons argilo-sableux gris brun, bioturbés à structure friable (horizon supérieur du sol actuel)

us 3. limons argileux bruns à structure agrégée (rares graviers à répartition hétérogène)

us 4,5 . argiles limoneuses jaune orangé très homogènes, à taches ferrugineuses (structure compacte, ces argiles comportent des sables grossiers et de rares inclusions aléatoires de graviers). Niveau azilien au sommet

us 6 . limons sableux oranges us 7. schiste altéré

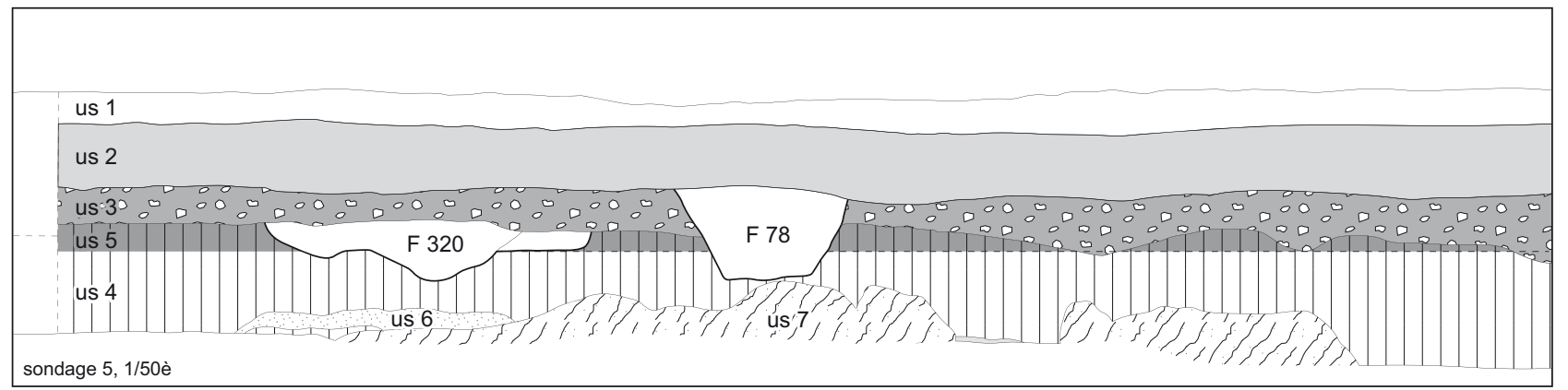

Fig. 9 - Détail de la coupe nord de la zone 5 (conception: R. Arthuis, Inrap; dessins: J.-F. Nauleau, Inrap).

assemblages archéologiques aziliens. En revanche, les prélèvements réalisés dans la zone 5 proviennent des niveaux 1 et 2 et de la partie inférieure du niveau 3.

\section{LE CONTEXTE PÉDOLOGIQUE}

Rappelons que l'évolution pédologique a été l'un des critères discriminant des différents niveaux sédimentaires décrits précédemment. Cette évolution est marquée par des traits différents selon les facteurs stationnels de chaque zone. Parmi ces traits, les imprégnations de fer, la compacité des sédiments et l'enrichissement en argiles sont concentrés dans les niveaux relativement profonds, notamment dans l'emballage sédimentaire du niveau azilien. Ces traits traduisent une stabilisation en place du sol relativement de longue durée (Avery, 1985; Bullock, 1985; Fedoroff, Courty, 1994; Sellami, 1999). En revanche, cette stabilité a été souvent interrompue par des phases de dégradation de courte durée, marquées par des niveaux de graviers. Ceci implique qu'une grande partie des sols a été partiellement tronquée. Seuls les horizons profonds des sols sont conservés grâce à leur développement pédologique générant une résistance à l'érosion (Ordaz, 1995; Sellami, 1999). En plus de ces remaniements entraînant des mou- vements latéraux de matériaux sédimentaires, les niveaux conservés ont été affectés par une dégradation pédologique en place. Celle-ci est marquée par une forte hydromorphie des matériaux se traduisant par une abondance des imprégnations ferrugineuses et par le blanchissement local de la masse du sol. Contrairement aux processus érosifs, pouvant entraîner le transfert latéral des assemblages archéologiques, les dégradations pédologiques affectent peu l'organisation des artefacts (Sellami et al., 2002). Leurs effets sont essentiellement concentrés sur la redistribution verticale des assemblages de silex et sur la décomposition des artefacts organiques.

\section{DYNAMIQUE DES SOLS ET ORGANISATION STRATIGRAPHIQUE}

Malgré la localisation du site au sein d'une seule et même unité géomorphologique matérialisée par une dépression, la position morpho-topographique varie sensiblement d'une zone à l'autre, voire au sein d'une même zone. Il en a résulté une accumulation et une évolution sédimentaire spécifiques, liées directement aux facteurs stationnels responsables des nuances stratigraphiques entre les différentes coupes. 


\section{LA ZONE 1}

La coupe de la zone 1 présente une alternance d'unités stratigraphiques d'épaisseurs variables. Leur continuité latérale peut traduire un fonctionnement globalement modéré des processus érosifs. En effet, l'absence de chenaux et de ravinements des surfaces des sols montre que la répartition des ruissellements de surface a été régulière, ce qui a limité leur canalisation, entraînant très souvent des creusements. Néanmoins, l'organisation morphologique et la composition des unités stratigraphiques sont différentes entre la base et le sommet de la coupe (fig. 7 et 10). À la base, les unités stratigraphiques 10, 11 et 12, composées par des matériaux essentiellement argileux, se distinguent par des surfaces ondulées. Elles traduisent des troncatures du sol induites par des transferts hydriques de faible énergie fonctionnant sur de longues durées (Wainwright, 1994). Ces troncatures sont le résultat de la répétition des transferts colluviaux exigeant une saturation temporaire du sol en eau. En revanche, la partie supérieure de la coupe, comprenant les unités 13 et 14, se distingue de la base par la nature des composants et par l'organisation globale des couches. Les matériaux sédimentaires sont nettement enrichis en matière organique. La limite entre les unités est presque rectiligne, ce qui s'explique par une accumulation rapide. L'érosion n'a pas affecté significativement les sols puisque les transferts hydriques, susceptibles d'entraîner des mouvements sédimentaires, ont été de faible énergie. L'analyse micromorphologique des unités 10 à 13 a montré les différents processus de leurs formations et surtout les conditions d'évolution qui ont permis, tantôt la stabilité, tantôt le remaniement des sols.

L'unité stratigraphique 10, homogène sur le terrain, se révèle très complexe. Elle est formée de limons argilosableux composés de quartz, de grès, de nodules de fer, de paillettes de schiste et de micas. Les sables sont bien altérés et souvent en voie de fragmentation. Cette composition traduit une dynamique faible de dépôt intégrant des inclusions aléatoires de sables indiquant des ruissellements de surface temporaires plus ou moins importants. À l'origine, ces derniers sont matérialisés par des faciès sableux relativement bien triés et bien matérialisés en surface du sol à l'instar des niveaux de galets (stone lines) reconnus dans différentes régions (Schwartz, 1990). Reconnus tous les deux comme des témoins de troncature des sols, les faciès sableux se caractérisent par une dynamique très faible qui n'affecte que la fraction fine du sédiment. Après leur dépôt, ces sables ont été réorganisés en place par les processus pédologiques, dont l'activité biologique au sens large.

Les variations minéralogiques s'expliquent par des sources d'alimentation sédimentaire différentes. Si les quartz, les feldspaths et les micas proviennent de l'altération du substrat et des apports éoliens soufflés de la vallée de la Loire, les nodules de fer et les paillettes de schiste, eux, sont dérivés de la dégradation en place de la roche mère schisteuse. L'ensemble est scellé par une abondante matrice argileuse répartie d'une manière hétérogène et incluant des organisations de sables. Les variations de structure de cette unité stratigraphique sont liées à l'évolution postdépositionnelle qui est également marquée par des traits pédo-sédimentaires. Ces variations témoignent de plusieurs phases de dépôts et de fonctionnement du sol. Les plus importantes sont matérialisées par des faciès spécifiques. En effet, malgré l'homogénéité globale de cette unité, trois faciès sédimentaires ont pu être distingués: les limons argileux homogènes, les limons argilo-sableux hétérogènes, et les limons argileux lavés (tabl. I). Les deux premiers alternent d'une manière régulière du bas vers le haut de l'unité, alors que le troisième est localisé au sommet. Les deux premiers se composent d'un mélange d'inclusions de paillettes de schiste, de sables grossiers et d'agrégats argileux témoignant d'une dynamique de dépôt relativement plus importante que celle des limons. Les agrégats argileux indiquent une reprise de sols pédologiquement bien développés alors que les revêtements argileux brunsrouges sont le témoin direct de la stabilisation de la surface du sol (Cremaschi, 1990; Curmi, 1987). En revanche, les revêtements massifs et mal triés traduisent une dégradation temporaire marquée par une saturation en eau du sol. La combinaison de ces deux types de traits reflète une évolution polyphasée du sol marquée par une alternance de phase stable et de phase de dégradation des conditions du milieu.

Le troisième faciès montre, par sa texture homogène, une dynamique régulière de dépôt à laquelle les apports éoliens ont largement contribué par l'apport des micas. L'absence de paillettes de schiste s'explique par l'enfouissement du substrat au moment de la formation de ce faciès alors que les organisations diffuses de limons lavés témoignent d'un drainage brutal du sol lié à la présence, dans ce dernier, d'un important volume d'eau entraînant une réorganisation en place des sédiments. Ce processus est identique à celui reconnu dans les milieux périglaciaires (Huijzer, 1993; Fedoroff, Courty, 1994; Sellami, 2002) : le gel du sol entraîne la formation de lentilles de glace qui, 


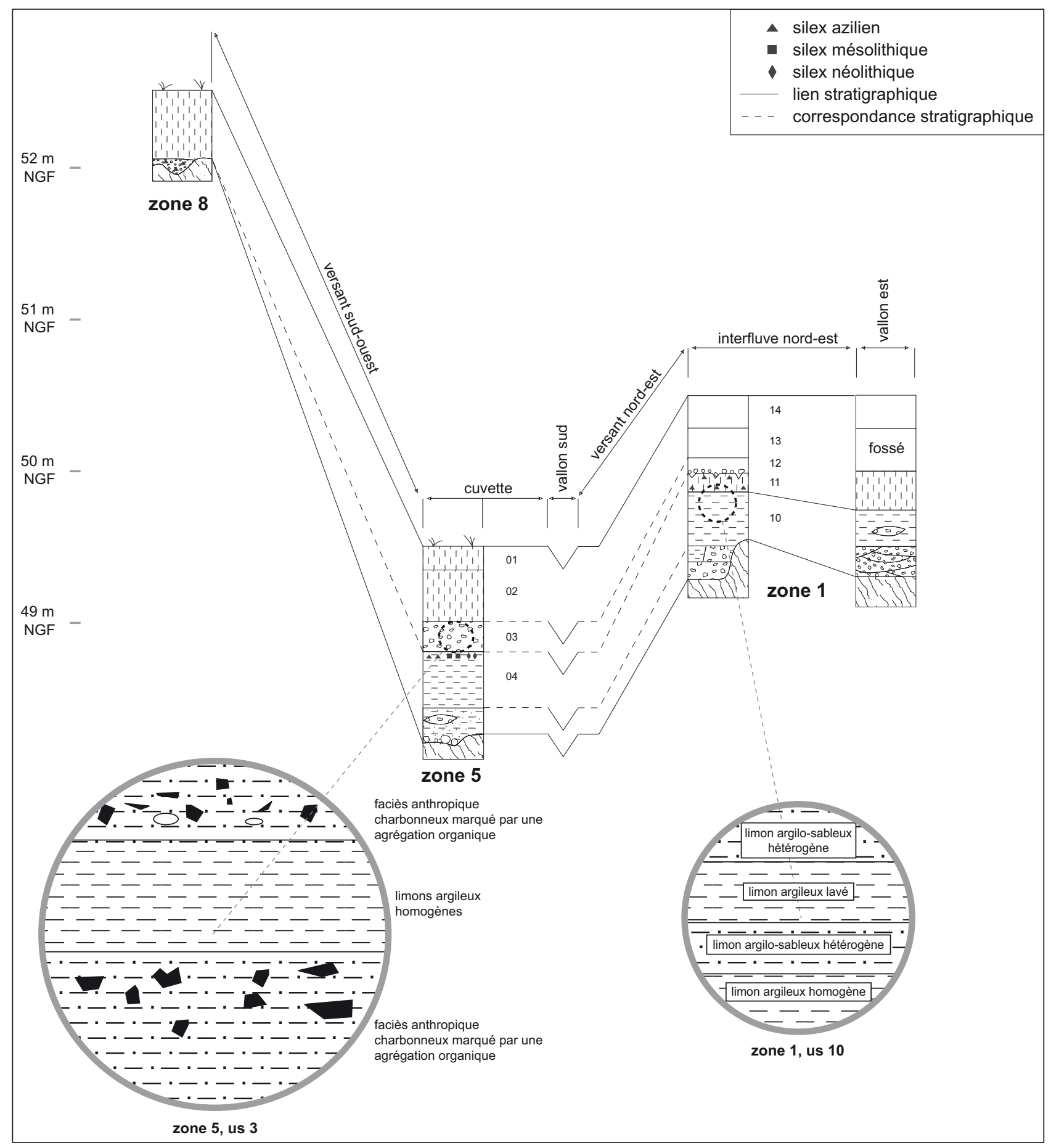

Fig. 10 - Liens et corrélations stratigraphiques des sédimentations de la cuvette des Chaloignes (zones 8, 5 et 1) et indications des observations micromorphologiques (conception: R. Arthuis, Inrap et F. Sellami, Inrap; dessins: J.-F. Nauleau, Inrap).

en fondant, libèrent brusquement un important volume d'eau dans le sol. Il en résulte une réorganisation interne des sédiments sans mouvement, particulièrement dans les endroits de faible pente. En revanche, dans les surfaces en pente, la saturation en eau du sol entraîne un mouvement colluvial dont la répétition peut provoquer des troncatures.

La prise en compte de l'ensemble de ces critères montre que l'unité 10 est combinée de plusieurs phases sédimen- 
Tabl. I - Caractérisation des faciès sédimentaires constituant la couche 10.

\begin{tabular}{|l|l|}
\hline \multicolumn{1}{|c|}{ Faciès sédimentaires } & \multicolumn{1}{c|}{ Caractérisation pédo-sédimentaire } \\
\hline Limons argileux homogènes & $\begin{array}{l}\text { - Texture bien triée } \\
\text { - Inclusions rares d'agrégats argileux } \\
\text { - Répartition homogène de la fraction fine et grossière } \\
\text { - Revêtements argileux dans la masse }\end{array}$ \\
\hline \multirow{2}{*}{ Limons argilo-sableux hétérogènes } & $\begin{array}{l}\text { - Texture non triée : mélange de sable, de nodules de fer et de paillettes de schistes et de micas } \\
\text { - Inclusions abondantes d'agrégats argileux } \\
\text { - Présence de fragments de revêtements argileux dans la masse }\end{array}$ \\
\hline Limons argileux lavés & - Ce faciès se distingue du premier par un lavage localisé des limons et par des organisations diffuses de limons lavés \\
\hline
\end{tabular}

taires. Les plus importantes sont celles inscrites dans les faciès superposés montrant une formation progressive de cette unité. Cette formation est marquée par une alternance d'apports sédimentaires de proximité, générés par de faibles colluvionnements, et d'apports un peu plus importants depuis les versants par ruissellement diffus. Les premières phases traduisent une relative stabilité de la surface du sol alors que les secondes reflètent une certaine dégradation des versants et l'affleurement du substrat. Ces différentes phases de sédimentation sont relativement longues, mais pas suffisamment pour permettre la distinction de pédogenèses spécifiques. Après leur dépôt, les sédiments ont été rapidement homogénéisés par deux processus majeurs: l'activité biologique reflétant un bon drainage du sol d'une part, l'hydromorphie traduisant une saturation temporaire du sol responsable du colmatage des sédiments et de leur ferruginisation présente sous forme nodulaire d'autre part.

L'unité stratigraphique 11, qui contient l'industrie lithique azilienne, est représentée par un seul faciès de type limono-argileux identique à celui reconnu dans l'unité précédente, indiquant ainsi que leur formation est liée à une même phase de sédimentation. Cette unité 11 se caractérise, néanmoins, par l'absence totale de paillettes de schiste et une abondance d'inclusions d'agrégats organiques souvent ferrugineux. Quelques fragments charbonneux (2 mm à $4 \mathrm{~mm}$ ) sont intégrés dans une masse limoneuse. Bien que ces inclusions soient concentrées au sommet, leur répartition est globalement aléatoire traduisant la complexité de la dynamique de dépôt. Elle est combinée d'apports éoliens et d'apports colluviaux générés par la thixotropie du sol. La thixotropie est définie comme la «propriété qu'ont certains corps très visqueux ou très humides de se liquéfier par agitation, puis de reprendre leur état initial par repos. Ainsi, certains terrains vaseux présentent cette propriété: on peut les fouler, puis ils deviennent fluides et on s'y enfonce; après repos, ils retrouvent leur rigidité initiale [...]» (Lozet, Mathieu, 1990). Ces colluvions de faible énergie expliquent l'absence des sables grossiers et de paillettes de schiste en relation avec la stabilité des versants.

La présence d'agrégats organiques ne peut être expliquée par les seuls processus naturels, car la matrice sédimentaire n'en présente aucune trace. Leur concentration mêlée à des fragments de charbons de bois au sein d'un seul faciès, d'environ $10 \mathrm{~cm}$ d'épaisseur, reflète une origine anthropique. Ces critères évoquent ceux décrits dans les phases actives de sols archéologiques (Gé et al., 1993; Sellami, 1999). La répartition aléatoire de ces agrégats est liée au fonctionnement des processus pédologiques de l'emballage sédimentaire tels que l'activité biologique et l'alternance saturation/dessèchement des sédiments. Ces deux processus ont entraîné un remaniement interne du sol. L'abondance de l'activité biologique traduit un bon drainage du sol témoin de conditions environnementales modérées, alors que la saturation du sol résulte d'une forte humidité du milieu. L'engorgement du sol qui en découle explique la ferrugénisation des sédiments et des agrégats organiques qui sont, de ce fait, bien consolidés.

Cette évolution pédologique a été à l'origine de la compaction du sol qui explique la structure massive de cette unité. Le comblement des vides cavitaires est le résultat des transferts sédimentaires et pédologiques internes dans le sol. Les organisations lenticulaires de limons sont les premiers traits de ces transferts avant la compaction totale du sol. Les argiles limoneuses sont réorganisées par la suite: leur faible organisation montre un dépôt rapide. Cette succession des traits reflète une évolution progressive de cette unité stratigraphique qui, d'après la structuration des traits argileux, n'a pas été remaniée depuis sa formation. Néanmoins, sa partie supérieure a été érodée, marquant une limite nette avec l'unité sous-jacente. Cette limite est caractérisée par une concentration de sables lavés témoignant de cette troncature qui a décapé les horizons supérieurs des sols. L'absence d'alignements de graviers et/ ou de galets signifie que cette troncature, générée par la 
répétition des ruissellements, a été progressive. La répartition aléatoire des sables, initialement sous forme d'un faciès sableux bien matérialisé, est le résultat de l'évolution pédologique post-dépositionnelle.

L'unité stratigraphique 12 est homogène. Elle est composée d'argiles limoneuses incluant des sables grossiers et des fragments organiques. La composition minéralogique est identique à celle des unités sous-jacentes, ce qui montre la même provenance sédimentaire. Néanmoins, l'absence de paillettes de schiste indique que le substrat schisteux a été enfoui au moment de la formation de cette unité. Sa distinction est fondée sur la couleur brune, l'abondance des argiles et surtout l'aspect organique des sédiments. La matière organique est présente sous deux formes. La première, humique, est intégrée dans les sédiments alors que la seconde est présente sous la forme de plages lenticulaires et d'agrégats épars dans la masse du sol. La présence de cette matière organique témoigne de la dégradation des restes végétaux en surface, puis de la migration du jus organique dans le sol entraînant la brunification du sédiment (Fedoroff, Goldberg, 1982). En revanche, sa présence sous forme d'organisations diffuses et d'agrégats est liée à l'apport de matériaux organiques, faisant souvent suite à l'anthropisation de la surface du sol. L'intégration de ces agrégats dans la masse sédimentaire résulte du remaniement en place du sol par des activités culturales marquées par les triages limono-argileux. Leur formation est liée au remaniement mécanique des sédiments provoquant l'effondrement de la structure sédimentaire et la désagrégation du sol (Sellami, 1999). Il en résulte un transfert vertical des particules à la suite de percolations rapides d'eau. Cette réorganisation du sol, purement anthropique, a été à l'origine de l'homogénéisation de cette unité à laquelle l'activité biologique a également contribué. La structure massive montre une relative stabilité de la surface du sol alors que l'absence de revêtements argileux indique une pédogenèse faible que justifie le rajeunissement du sol par des apports sédimentaires continus.

\section{LA ZONE 5}

La coupe de la zone 5 (fig. 9), située au centre de la dépression, montre une superposition d'unités stratigraphiques latéralement continues, indiquant que les processus érosifs n'ont pas été importants. En effet, les transferts hydriques ont été répartis sur toute la surface, limitant ainsi leur canalisation et donc leur capacité d'érosion. Seuls les fosses et les fossés d'origine anthropique ont entraîné des discontinuités des unités stratigraphiques sans pour autant perturber totalement la lecture stratigraphique. Les transferts hydriques n'ont pas été assez importants pour provoquer la formation de chenaux. En revanche, leur dynamique, bien que de faible énergie, a été suffisante pour décaper partiellement et progressivement les sols, provoquant une absence des horizons supérieurs.

L'analyse micromorphologique a porté essentiellement sur l'emballage du niveau azilien incluant son support et sa couverture sédimentaire. Les faciès sédimentaires reconnus au sein de cette coupe évoquent ceux décrits dans la zone 1 (fig. 10). Nous présentons ici les variations sédimentaires et pédologiques spécifiques à la zone 5, en tentant d'expliquer les processus qui en sont à l'origine.

L'unité stratigraphique 4 est représentée par des limons argilo-sableux hétérogènes identiques à ceux du faciès déjà décrit au sommet de l'unité stratigraphique 10 dans la zone 1. Cette ressemblance, tant sédimentaire que pédologique, représente un critère de corrélation pertinent. Il apparaît comme très fiable, dans la mesure où ces deux unités représentent le support naturel de l'occupation azilienne, les assemblages lithiques se situant à chaque fois à leur sommet. Malgré leur similitude globale, l'unité stratigraphique 4 de la zone 5 se distingue de l'unité stratigraphique 10 de la zone 1 par une diminution sensible des paillettes de schiste en faveur des sables grossiers et des graviers anguleux et par des plages sableuses bien individualisées et partiellement lavées. Cette diminution est liée à la localisation de cette unité sur un replat plus large. Ceci a favorisé un fonctionnement long de la dynamique de dépôt, montrant que nous sommes au sein d'une zone relativement bien éloignée du versant en pente sus-jacent (interfluve sud-ouest). En revanche, la présence de sables et de graviers témoigne de ruissellements relativement importants dont la répétition est susceptible de tronquer le sol. Bien qu'aléatoirement redistribués dans le sol, ces sables graveleux forment initialement un niveau bien individualisé. Leur origine est associée aux processus périglaciaires inscrits au sein de l'unité stratigraphique 10 dans la zone 1. Cette corrélation est fondée sur le fait que les processus liés au gel-dégel du sol peuvent effectivement provoquer ce type d'érosion dont l'impact sur la surface du sol est souvent fonction des facteurs stationnels. Sur les endroits topographiquement plats, la saturation en eau du sol, générée par les fontes du gel, entraîne des triages texturaux presque sans mouvements des sédiments. Ce type de triage, caractérisant dans la zone 1 les limons argileux de l'unité stratigraphique 10, indique que nous sommes en présence d'une 
période périglaciaire. En revanche, dans les endroits en pente, ces mêmes conditions ont favorisé des ruissellements diffus à l'origine de transferts sédimentaires et de troncatures des sols telles que celles matérialisées par des organisations sableuses dans la zone 5. Ces organisations ont été redistribuées par le brassage biologique des sédiments qui explique leur répartition aléatoire dans la masse.

L'unité stratigraphique 3 est représentée par des limons argileux homogènes, pédologiquement bien développés. Leurs caractéristiques pédo-sédimentaires rappellent avec précision celles de l'unité 12 reconnue dans la zone 1. Cette ressemblance montre la continuité d'une seule et même unité sédimentaire depuis la zone 5 jusqu'à la zone 1 , alors que les variations pédo-sédimentaires sont générées par le fonctionnement de facteurs stationnels distincts entre ces deux zones. La topographie et la géomorphologie s'affichent comme des facteurs principaux de variation du milieu sédimentaire. L'épaisseur relativement importante de cette unité 3 (environ $20 \mathrm{~cm}$ ) a été favorisée par la régularité de la surface du sol au sein de la zone 5. La présence d'organisations et d'agrégats organiques au sein de la matrice limoneuse homogène s'explique par des activités anthropiques relativement intenses puisque ces organisations sont réparties en deux micro-faciès. L'un, à la base, est très riche en éléments organiques et en microfragments de silex anguleux; l'autre, au sommet, intègre, en plus, des fragments charbonneux. La matérialisation de ces deux micro-faciès traduit deux occupations différentes séparées par un faciès limoneux totalement dépourvu de matière organique marquant une limite diffuse et progressive. Cette limite montre que les transferts sédimentaires et les processus pédologiques ont fonctionné simultanément, permettant une homogénéisation régulière des sédiments.

L'unité stratigraphique 2 ne présente aucune différence permettant de la distinguer de l'unité 13 matérialisée au sein de la zone 1. Rappelons néanmoins que ces deux unités stratigraphiques, formées dans deux zones différentes, sont largement anthropisées. Elles sont développées sur une épaisseur importante de sol, ce qui montre la stabilité de la surface du sol et la diminution des transferts sédimentaires sur la totalité du site.

\section{L'ÉVOLUTION DES SOLS ET LEUR ANTHROPISATION}

Malgré la variation des conditions morpho-topographiques stationnelles spécifiques à chacune des zones, la dynamique globale du site apparaît homogène, ce qui est à l'origine de la conservation des faciès sédimentaires identiques d'une zone à l'autre (fig. 10). Sur la base des résultats détaillés des micro-faciès présentés ci-dessus, nous tenterons une reconstitution de la dynamique des sols et sa répercussion sur la répartition latérale des unités stratigraphiques et des niveaux archéologiques.

\section{ÉVOLUTION LATÉRALE ET CORRÉLATION DES FORMATIONS SÉDIMENTAIRES}

La variation des facteurs stationnels à l'échelle du site a généré des nuances pédo-sédimentaires des matériaux et une conservation discontinue des unités stratigraphiques. Ainsi, bien que les conditions d'évolution du milieu naturel soient temporairement les mêmes, les faciès sédimentaires qui en résultent sont parfois totalement différents d'une zone à l'autre selon les structures géomorphologiques locales. Par exemple, la dégradation périglaciaire, dont les traits sont bien conservés dans la zone 1, est totalement absente dans la zone 5 où elle est plutôt inscrite par une érosion (fig. 11). Cette variation latérale des unités stratigraphiques, pourtant formées sous les mêmes conditions du milieu, reste l'une des caractéristiques de l'évolution des sites de plein air (Sellami, 1999). La répercussion de cette évolution sur la conservation dépasse largement celle des sols naturels pour se généraliser sur la préservation des niveaux et des assemblages archéologiques. La conservation des structures anthropiques ne peut donc être extrapolée, dans ce cas, sur la totalité d'une surface d'occupation, à moins qu'elle ne soit validée sur les différents endroits du site. Malgré la variation des critères sédimentaires, tels que l'épaisseur, l'organisation et les nuances texturales distinguant les faciès, la majorité des unités stratigraphiques sont en parfaite corrélation entre la zone 1 et la zone 5 (fig. 10). Cette variation résulte essentiellement des facteurs stationnels, tel que la pente, agissant directement sur les processus sédimentaires et pédologiques. Ces facteurs sont en grande partie responsables de plusieurs troncatures qui ont notamment décapé les horizons supérieurs des sols. Rappelons que l'une des plus importantes est celle marquant la transition abrupte entre les unités stratigraphiques 11 et 12 au sein de la zone 1. Cette troncature a été néanmoins très localisée, puisqu'elle n'a pas affecté la totalité du site. Sa présence n’a pas été matérialisée au sein de la zone 5 où la surface du sol est régulière. 


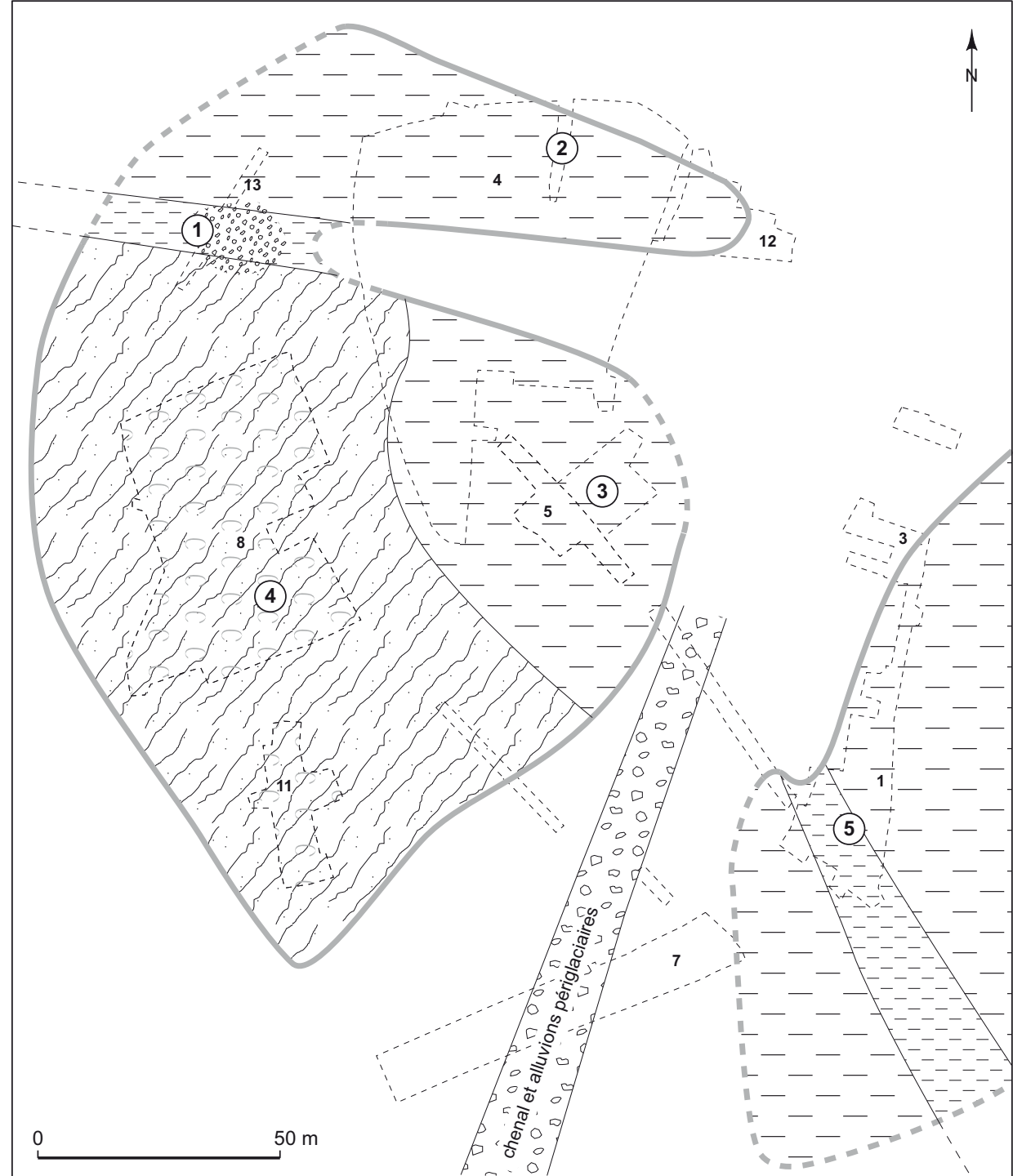

schiste nu ou recouvert d'un sol peu épais. Microtopographie « accidentée », avec notamment de petites cuvettes dépôts de versants (fine essentiellement)

dépôts fins de fond de vallon

dépôt en masse de blocs de quartz

- limite correspondant à la troncature des niveaux aziliens

- - limite supposée correspondant à la troncature des niveaux aziliens
(1)

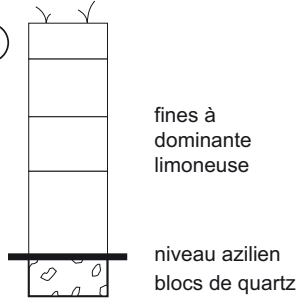

(2)

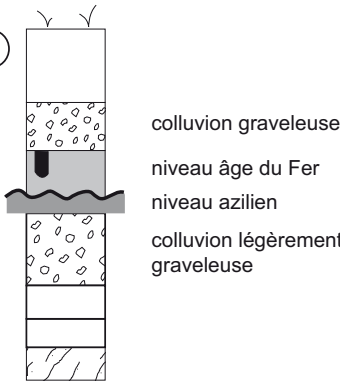

(3)

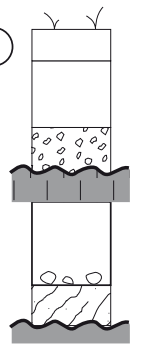

niveau " cultivé " (tessons $\mathrm{XI}-\mathrm{XIl}{ }^{\mathrm{e}} \mathrm{s}$.)

niveau azilien,

mésolithique,

néolithique final

dépôts périglaciaires

(4)

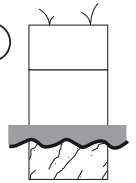

travaux agricoles

colluvion

niveau azilien

(5)

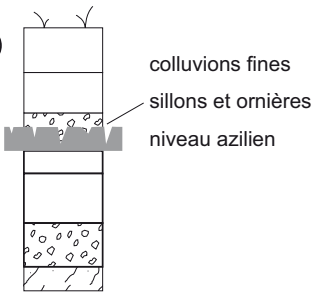

Fig. 11 - Représentation de la couverture sédimentaire résiduelle azilienne (conception: R. Arthuis, Inrap; dessins:J.-F. Nauleau, Inrap).

\section{NATURE DES DYNAMIQUES ET DES CONDITIONS DE FONCTIONNEMENT DU SITE}

Malgré la conservation attestée des sols et des niveaux archéologiques au sein de ce site, son évolution globale, inscrite dans les différentes unités stratigraphiques, a été hétérogène et cyclique. La discontinuité de l'enregistrement stratigraphique n'est qu'un résultat de cette hétérogénéité, en relation directe avec la nature des conditions du milieu. Cette évolution, relativement complexe, a été inscrite selon la nature morpho-topographique, sédimentaire et pédologique du milieu. L'identification et la hiérarchisation des traits pédo-sédimentaires et leur confrontation avec les données de terrain ont permis de distinguer, au sein des 
dépôts, différentes phases d'évolution que nous tenterons de présenter de la plus ancienne à la plus récente.

\section{Une phase d'érosion du maximum glaciaire}

Cette phase se traduit par d'importantes conséquences sur le bilan sédimentaire du site. La totalité des sols pléistocènes a été érodée, entraînant une remise à zéro de la mémoire sédimentaire (fig. 12). Il ne subsiste pratiquement plus rien des couvertures sédimentaires antérieures à celles attribuables au Tardiglaciaire par la présence du niveau azilien. Cette érosion a entraîné le dépôt d'un niveau hétérogène composé de galets, de blocs quartzeux et de sables au contact du substrat, exclusivement au fond de la cuvette (fig. 6). Ce dépôt témoigne de l'ampleur de cette érosion durant laquelle la cuvette a canalisé la dynamique hydrique. La dynamique hydrique, issue de la fonte des glaces et des neiges, fonctionnant sur les versants n'a permis aucun dépôt durant cette phase. Cette érosion attestée sur presque la totalité de la surface du site suppose une mise à nu des versants dominants le site, ce qui peut expliquer le décapage des sols anciens. En tout état de cause, les processus de ruissellement à l'origine de cette érosion semblent fonctionner sur une longue période, car les sols ont été totalement érodés. En effet, dans le cas d'une érosion rapide, la dynamique hydrique, souvent canalisée, n'affecte que localement les sols, ce qui entraîne une préservation discontinue.

\section{Une phase intermittente de stabilisation et de recharge sédimentaire}

La mise en place de la couverture sédimentaire fine commence immédiatement après le dernier maximum glaciaire et se termine avant la fin du Tardiglaciaire comme l'atteste la présence des silex aziliens dans la sédimentation sommitale tardiglaciaire. De fait, le niveau azilien, constitué de silex organisés sur une quinzaine de centimètres d'épaisseur, a servi de repère chronostratigraphique permettant d'évaluer sur le terrain les variations de la recharge sédimentaire d'un endroit à l'autre. Au-delà des apports latéraux, combinés de colluvions et d'alluvions, la dynamique éolienne a significativement contribué à la formation des sols (fig. 12). Malgré l'abondance de ces apports provenant d'horizons situés au-delà des limites du bassin versant, leur redistribution locale par les dynamiques alluviales et colluviales précitées n'a pas permis la formation de sol éolien typique. Cette redistribution explique également l'intégration de quelques éléments grossiers au sein des limons. Néanmoins, cette réorganisation n'implique pas l'évacuation totale de ces matériaux qui sont piégés, faute de dynamique suffisante, par les couverts herbacés. Ces derniers couvrent en effet rapidement les sols en bénéficiant de leur richesse minéralogique. La répétition de ces piégeages est en grande partie responsable de la recharge sédimentaire avant et après l'occupation azilienne. Ce processus a été alors responsable de la formation du support naturel du niveau d'occupation azilien et de l'emballage sédimentaire des assemblages archéologiques. Durant cette phase, le profil topographique revient progressivement à l'équilibre limitant ainsi significativement les transferts sédimentaires. Il en résulte un comblement des ravinements creusés lors de la première phase. Cet équilibre du profil a entraîné une diminution progressive des dépôts au sein même du site devenant alors une zone de transit des matériaux. Ce phénomène explique en partie la faible épaisseur de la stratigraphie.

\section{Une phase de fonctionnement pédologique}

Le fonctionnement des processus pédologiques a déjà débuté d'une manière fluctuante au cours des épisodes de stabilisation de la phase précédente. Néanmoins, cette pédogenèse coïncide avec la diminution des apports sédimentaires. Il faut cependant préciser que ces derniers n'ont pas été totalement arrêtés. Leur fonctionnement a d'ailleurs été à l'origine de phases sédimentaires faibles mais continues, à l'origine du rajeunissement régulier du sol. Ce rajeunissement est également dû à la pente faible de la surface du sol permettant le fonctionnement simultané des processus sédimentaires d'une part, pédologiques d'autre part. Cette combinaison explique l'absence de formation de sol caractéristique. Seule la matérialisation de traits pédologiques tels que l'agrégation et les revêtements argileux traduit ce fonctionnement, marquant néanmoins des épisodes de stabilisation temporaire des conditions du milieu. C'est durant l'un de ces épisodes que l'occupation azilienne a eu lieu.

\section{Une phase de dégradation du sol sous des conditions périglaciaires}

Rappelons que l'équilibre du profil topographique survenu durant la deuxième phase a nettement limité les dépôts sédimentaires expliquant la faible épaisseur des sols. De ce fait, cette dégradation périglaciaire, affectant principalement les couches sus-jacentes du niveau azilien, a 

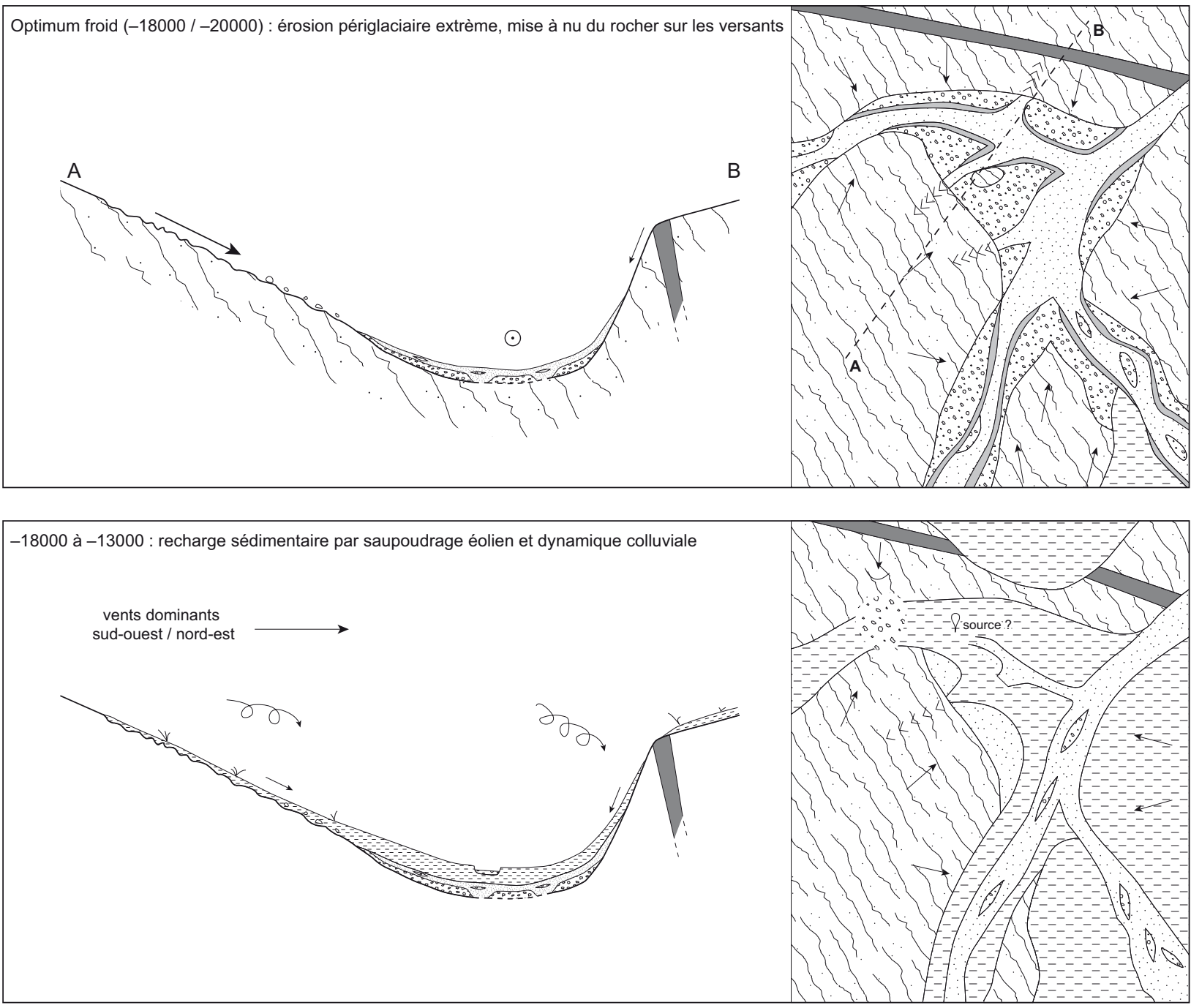

Occupation azilienne : paysage minéral hérité du Würm ; couverture sédimentaire discontinue et d'épaisseur très variable ; stabilité sédimentaire, pédogénèse, sol bien drainé
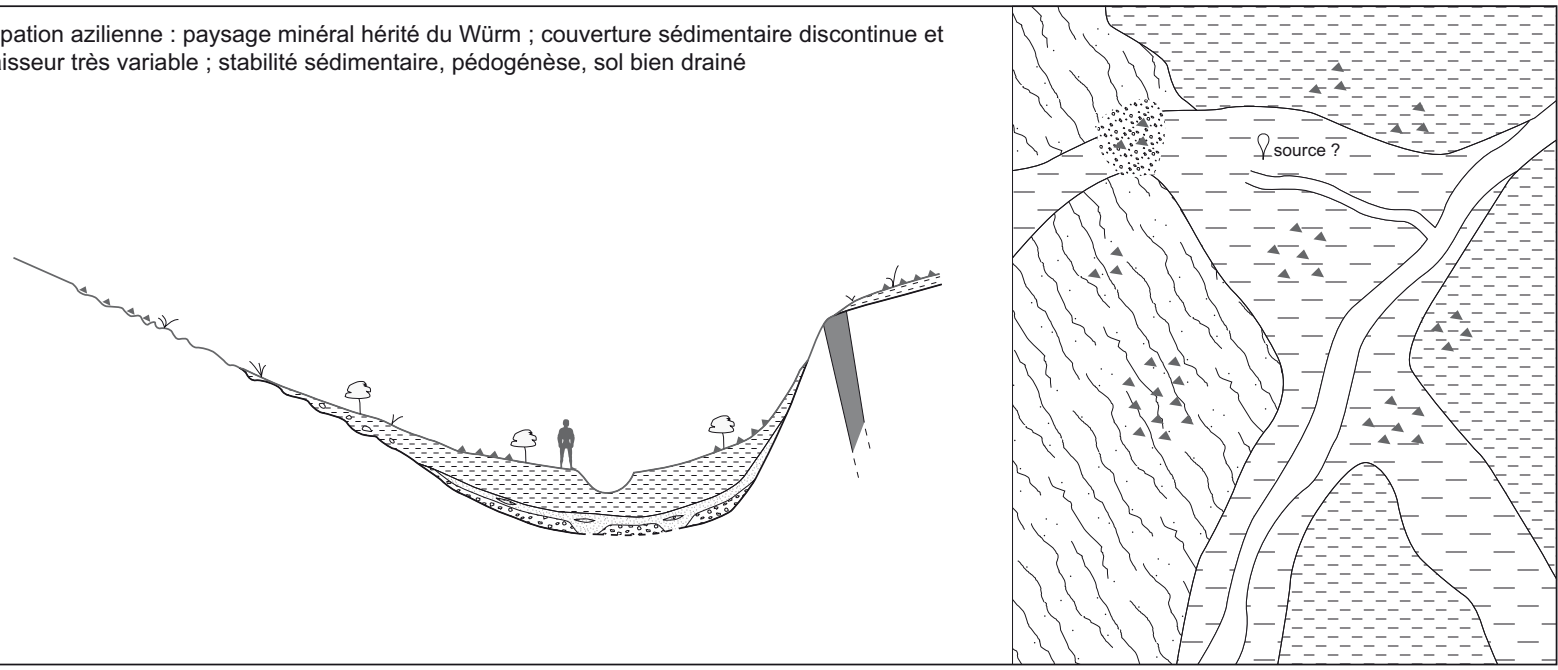

Fig. 12 - Évolution géomorphologique de la cuvette des Chaloignes en trois instantanés couvrant le Pléniglaciaire et le Tardiglaciaire (conception: R. Arthuis, Inrap; dessins: J.-F. Nauleau, Inrap). 
légèrement atteint l'emballage sédimentaire des assemblages aziliens pourtant nettement plus anciens. L'inscription de cette dégradation dans le sol traduit le développement d'un gel relativement profond pouvant être corrélé avec l'épisode du Dryas récent. En effet, cette dégradation, largement identifiée dans différentes régions du globe par l'étude des fluctuations du niveau des lacs dans le Jura (Magny, 1995a et 1995b), des sols et des paléosols dans le Nord de la France (Van Vliet-Lanoë, 1987 et 1992), des stratigraphies archéologiques dans le sud-ouest de la France (Sellami 1999), des mollusques continentaux en Bourgogne (Puissegur et al., 1994), des pollens sur le site de la Grande Pile (Woillard, Mook, 1982), des courants océaniques dans l'Atlantique Nord (Bond et al., 1993; Smith et al., 1997) et des fluctuations des glaces au Groenland (Bond et al., 1993; Chappellaz et al., 1993), représente l'épisode le plus froid qui a significativement affecté les sols. Au cours de cet épisode, ceux-ci ont subi des réorganisations internes responsables de la formation des triages texturaux. Elles peuvent également être à l'origine des mouvements verticaux d'éléments relativement grossiers de la taille des graviers et des galets. Ces mouvements peuvent naturellement affecter les vestiges archéologiques de même taille. Malgré tout, l'organisation du sol n'a pas été fondamentalement perturbée, bien qu'il faille admettre quelques transferts liés notamment aux processus pédologiques. À la fin de cet épisode, le sol a été appauvri à cause de transferts sédimentaires; ils furent liés à la fonte des glaces et des neiges déposées lors de la dégradation périglaciaire. Il en a résulté une abondance des ruissellements qui ont entraîné une troncature partielle des sols, notamment les horizons superficiels suffisamment fragilisés par le gel. Cette troncature marque une transition nette vers les sols sus-jacents. Elle est responsable de la formation des niveaux graveleux à galets souvent discontinus et parfois redistribués dans la masse du sol.

\section{Une phase d'apports et de remaniements colluviaux}

Cette phase, matérialisée au sommet de la stratigraphie, est marquée par l'abondance des apports limoneux au détriment des sables notamment grossiers (fig. 13). Ce déséquilibre textural traduit la dominance de la dynamique colluviale responsable de l'homogénéisation du sol et des inclusions de graviers et de galets au sein de la masse du sol. Ces inclusions individuelles résultent de processus de remaniement, notamment ceux mécaniques liés aux activités culturales. Ces dernières ont entraîné une répartition homogène des remaniements alors que les mouvements sédimentaires sous l'effet de la saturation du sol sont concentrés dans les endroits en pentes. Ces transferts sont responsables de l'épaisseur du sol superficiel dans les zones favorables à la sédimentation. L'importance de l'influence anthropique est en grande partie responsable de la forte couverture sédimentaire holocène qui a largement contribué à la préservation du niveau azilien.

\section{FONCTIONNEMENT DYNAMIQUE DU SITE ET CONDITIONS DE FOSSILISATION}

L'absence d'une grande partie de la stratigraphie pléistocène n'a pas permis d'appréhender l'évolution des sols antérieurs à celui du Tardiglaciaire. Il est donc difficile de discuter du fonctionnement naturel de la cuvette à la période du maximum glaciaire (vers $19000 \mathrm{BP}$ ) qui, rappelons-le, a connu une vidange totale des formations sédimentaires du site. La prise en compte de cette vidange a été fondée sur la mise à nu du substrat et sur les traces d'érosion importantes, à défaut de la caractérisation des sols dont la présence reste à vérifier en dehors de la zone du site. Il est possible que le site soit successivement nettoyé par les dynamiques hydriques plus ou moins longues générées au cours des périodes glaciaires. Dans ce cas, la cuvette des Chaloignes a probablement fonctionné activement comme un bassin versant qui a longtemps alimenté le ruisseau de la Fontaine de Mont. Un tel fonctionnement pouvait significativement éroder les sols ou encore empêcher leur formation dans le cas de la présence d'une dynamique hydrique permanente. Ce phénomène pourrait être responsable de l'absence de traces de sols correspondant aux épisodes pléniglaciaires. En revanche, après la période du maximum glaciaire, les processus sédimentaires d'une part, érosifs d'autre part se sont orientés vers un équilibre progressif permettant une stabilisation temporaire des sols. Sur la base des rythmes sédimentaires, deux phases d'évolution du site peuvent être distinguées: une phase de fonctionnement purement naturel et une phase de fonctionnement sous l'influence du facteur anthropique (agriculture).

La première phase a été marquée par une stabilité relative et temporaire du sol qui est caractérisé par une pédogenèse peu développée. Ce faible développement a été le résultat d'un fonctionnement simultané des processus sédimentaires et pédologiques. Les apports sédimentaires, pourtant de faibles distances, ont généré un rajeunissement régulier du sol qui n'a pas pu encaisser continuellement les effets de l'évolution pédologique. En effet, les sédiments provenant des versants sont en grande partie arrachés direc- 

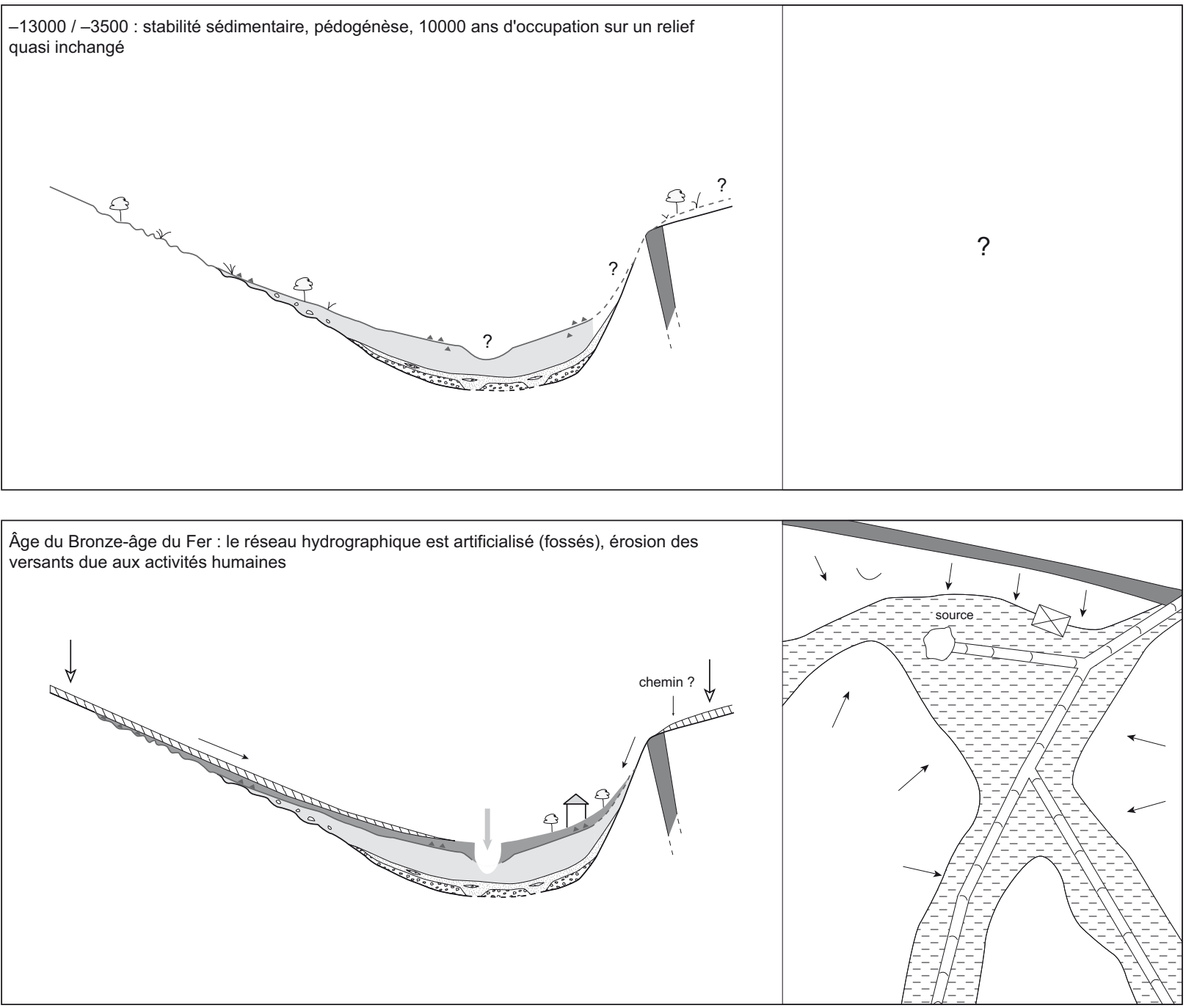

État actuel : mauvais drainage, prairie de milieu humide, présence d'une mare. Vallons est et ouest désactivés à la suite du creusement des fossés bordiers de la route ; vallon sud comblé

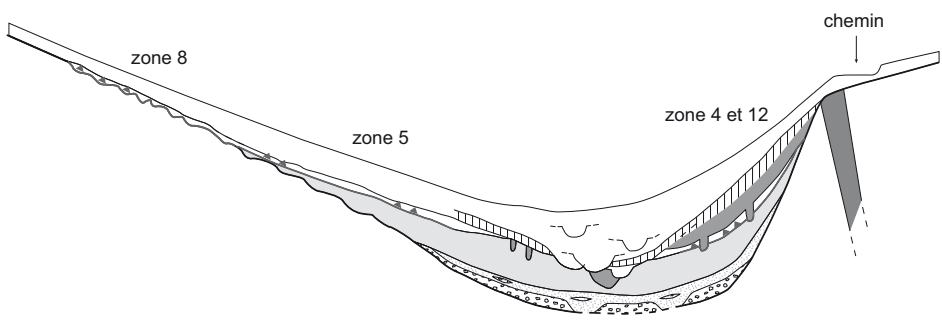

Fig. 13 - Évolution géomorphologique de la cuvette des Chaloignes après les occupations aziliennes, en trois instantanés couvrant l'Holocène (conception: R. Arthuis, Inrap; dessins: J.-F. Nauleau, Inrap). 
tement du substrat, ce qui leur confère un aspect peu pédogénéisé. Cette dynamique montre que nous sommes au sein d'un milieu sédimentaire où la pédogenèse a été discontinue sous l'effet des apports sédimentaires latéraux. Durant les épisodes de stabilisation de la surface du sol, le fonctionnement des processus pédologiques a été important, ce qui a permis d'ailleurs d'identifier la nature globale des conditions du milieu. Si cette alternance des processus sédimentaires et pédologiques semble être responsable du renouvellement sédimentaire au sein du site, elle a également influé sur la fossilisation des assemblages archéologiques. En effet, l'accumulation sédimentaire, plus abondante que les transferts depuis le site, a permis l'enfouissement plus ou moins rapide des assemblages anthropiques. La progression de ces apports essentiellement limoneux et de faible énergie n'a pas généré de perturbations significatives au sein de la cuvette des Chaloignes. En revanche, les pièces lithiques préhistoriques initialement abandonnées sur les versants peuvent être transférées avec les sédiments et arriver progressivement jusqu'à un endroit favorable au dépôt sédimentaire. Dans ce cas, elles sont intégrées individuellement dans la masse du sol, ce qui risque de les amalgamer aux éléments des locus en place, en laissant croire à une évolution stratigraphique de ces derniers (Sellami, 1999). Ces critères montrent que nous sommes en présence d'un niveau d'occupation dont l'emballage sédimentaire peut comporter des artefacts en position secondaire, sans relation directe avec ce niveau d'occupation bien qu'ils soient de la même période chronologique. La discussion ultérieure nous montrera que les vestiges aziliens sont datés par la chrono-typologie de l'Azilien récent, soit la seconde moitié de l'interstade Alleröd (XII ${ }^{\mathrm{e}}$ millénaire avant notre ère) si on a recours à des analogies typologiques. Autour de ces pièces, la mise en place d'un sol témoigne bien d'une amélioration climatique, qui intervient après des accumulations sédimentaires sous conditions périglaciaires. Logiquement, la troncature postérieure aux occupations aziliennes pourrait être corrélée au Dryas récent et au retour de conditions climatiques froides. Avec les difficultés inhérentes aux contextes sédimentaires et climatiques régionaux, cette succession «climat périglaciaire - amélioration climatique - péjoration climatique» évoque à n'en pas douter le Tardiglaciaire, avec son épisode terminal le Dryas récent. Il n'est évidemment pas possible de savoir si cette amélioration climatique intègre ou non l'interstade Bölling.

La seconde phase - entièrement datée de l'Holocène se distingue par l'importance des apports sédimentaires. Le fonctionnement dynamique ne diffère pas sensiblement de celui évoqué précédemment. Néanmoins, les accumulations sédimentaires apparaissent relativement hétérogènes : les inclusions de graviers et de galets y sont abondantes, ce qui traduit l'importance des dépôts. Les différents faciès reconnus en leur sein se distinguent par des traits pédosédimentaires traduisant un remaniement mécanique du sol résultant des activités culturales. Ces bouleversements répétés fragilisent la structure du sol, et entraînent des mouvements sédimentaires relativement importants selon la pente du sol, particulièrement lors de sa saturation en eau. La répétition de ce processus explique la dilatation de la séquence stratigraphique holocène correspondant globalement à cette phase. Malgré l'importance de ces mouvements sédimentaires, leur impact reste très modéré sur la conservation du sol et de ce qu'il comporte en termes d'assemblages archéologiques. En effet, les facteurs stationnels caractérisant le site sont plus favorables à la sédimentation qu'à l'érosion et le profil topographique du site est bien équilibré à l'Holocène.

Malgré la distinction de ces deux phases, les principaux mécanismes déclenchant les mouvements sédimentaires sont semblables, même s'il faut retenir l'influence sérieuse du facteur anthropique au cours de la seconde phase. Les transferts sédimentaires sont générés par des processus colluviaux plus ou moins importants selon le profil topographique et l'état hydrique des sols. Pendant la première phase décrite plus haut, une partie des apports a transité par le site pour être évacuée par le ruisseau de la Fontaine de Mont. En revanche, durant la seconde phase, la dynamique hydrique a sensiblement diminué, ce qui a favorisé l'accumulation des sédiments et l'arrêt presque total des transferts vers la vallée. Ces phases ont affecté différemment la couverture sédimentaire. Leurs dynamiques sédimentaires ont varié latéralement en fonction de leur énergie et de la morphologie de la surface du sol. Elles se sont ainsi répercutées sur le fonctionnement du ruisseau de la Fontaine de Mont dont l'évolution est en étroite relation avec la dynamique sédimentaire du site. En effet, l'activité de ce ruisseau est essentiellement contrôlée par la pluviométrie.

Deux types de dynamiques majeures peuvent être distingués en relation avec les conditions climatiques et environnementales.

- Une dynamique érosive peut être associée à des phases de dégradation climatique. L'importance du ruissellement qui en résulte peut influencer sur la régularité de la surface du sol et sur le fonctionnement du ruisseau de la Fontaine de Mont qui canalise les eaux de ruissellement. Il peut ainsi 
atteindre son activité maximale, ce qui entraîne le transfert des sédiments depuis le site vers la vallée. Les sols formés sur les versants dominant le site sont, de ce fait, totalement érodés, entraînant la mise en surface du substrat schisteux. Bien que temporaire, cette mise en surface a contribué significativement à l'importante altération des schistes que l'on peut observer actuellement.

- Une dynamique d'accumulation sédimentaire se produit lors des phases de stabilisation climatique durant lesquelles la surface du sol est stable. Elle permet la régénération du sol. Le site fonctionne ainsi comme un milieu stable, où l'accumulation sédimentaire, provenant des versants, est importante mais calme et progressive. Une telle dynamique est à l'origine des formations limono-argileuses homogènes, représentant la majeure partie des sédiments sur le site. Leur hydromorphie reflète l'engorgement temporaire des sols.

Considérant la variation des facteurs stationnels d'un endroit à l'autre du site, les phases précitées ne peuvent pas forcément être conservées sur toute la surface du site. L'évolution différentielle de la surface du sol a entraîné une répartition hétérogène des faciès en fonction des structures géomorphologiques locales. Ainsi, la phase de dégradation périglaciaire est marquée dans la zone 1 par un sol gelé bien conservé, alors qu'elle est inscrite dans la zone 5 par une troncature du sol. De la même manière, la phase d'érosion bien marquée sur la zone 1 au-dessus de l'occupation azilienne n'est nullement visible sur la zone 5. Cette variabilité latérale des sols reste ainsi une caractéristique des sites de plein air. Elle est également valable pour les niveaux d'occupations.

\section{CONGLUSION}

La conservation du site des Chaloignes s'explique parfaitement par la nature des conditions géomorphologiques et topographiques qui ont contrôlé la dynamique sédimentaire. Le dépôt des vestiges aziliens s'est fait lors d'une phase d'amélioration climatique, qui succédait à des dépôts complexes sous climat froid. Les interstades de Bölling et d'Alleröd correspondraient évidemment à cette partie supérieure de la stratigraphie; l'attribution à l'Azilien récent du matériel lithique ne laisse que la solution de l'interstade d'Alleröd (11800-10800 BP/12000 à 11000 avant notre ère). Malgré la dégradation temporaire des conditions du milieu au Dryas récent, le site n'a pas été totalement érodé. Quelques modifications sont cependant enregistrées au-dessus des locus aziliens.
Outre la morphologie de la cuvette qui favorise la sédimentation, les dégradations du milieu ont été moins importantes durant le Tardiglaciaire et l'Holocène que durant le Pléniglaciaire. D’un point de vue archéologique, le degré d'anthropisation des niveaux archéologiques a été important, ce qui explique la conservation de la matrice anthropique. Néanmoins, la conservation de ces niveaux peut se révéler variable d'un endroit à l'autre du site. Il est, de ce fait, possible que les assemblages du niveau azilien, qui sont très bien préservés dans la zone 5 par exemple, soient réorganisés dans d'autres zones. Cette réorganisation peut d'ailleurs davantage affecter l'emballage des assemblages archéologiques que les objets eux-mêmes.

F. S. et R. A.

\section{LES INDUSTRIES LITHIQUES AZILIENNES : MATÉRIAUX ET TECHNIQUES}

\section{PRÉSENTATION DES DONNÉES}

Qu'ils soient posés sur des limons ou sur le schiste altéré, les locus aziliens des bords de la cuvette (zones 1, 3, 8 et 11) sont à la fois peu étendus en surface et peu épais (entre $0,10 \mathrm{~m}$ et $0,15 \mathrm{~m}$ ) (fig. 14). Dans la cuvette, les silex de la zone 5 se repartissent sur une épaisseur plus forte (environ $0,20 \mathrm{~m}$ ), mais il s'agit justement de zones d'interprétations ambiguës. L'étude de l'industrie lithique bénéficie donc de conditions optimales de présentation si on les compare aux contextes des sites préhistoriques de plein air sur le Massif armoricain, avec ici une multiplication des collections homogènes (tabl. II). L'acidité des sols a entraîné une dissolution rapide des ossements d'animaux et de tout autre vestige d'origine organique, la totalité de l'information doit donc être extraite d'une lecture technique et spatiale des pierres taillées. Il n'existe malheureusement pas de traces d'aménagements domestiques de l'espace pour aider à comprendre l'organisation générale du site. Une structure azilienne qui s'apparenterait à une structure de combustion, a été repérée. Elle se situe à l'est du site, dans le locus 4 de la zone 3 , en limite de la surface azilienne homogène. Il s'agit d'un ensemble de quartz brûlés. Aucune trace charbonneuse n'était associée à ces pierres, qui ne sont pas structurées; elles semblent démantelées, posées à même le sol, sans creusement ni superposition. Elles correspondent peut-être au rejet d'une structure de combustion voisine, qui a pu être totalement lessivée. Notons que sur le site Federmesser de Rekem en Belgique, les quartz sont interprétés comme des pierres de cuisson («cooking stones»; 


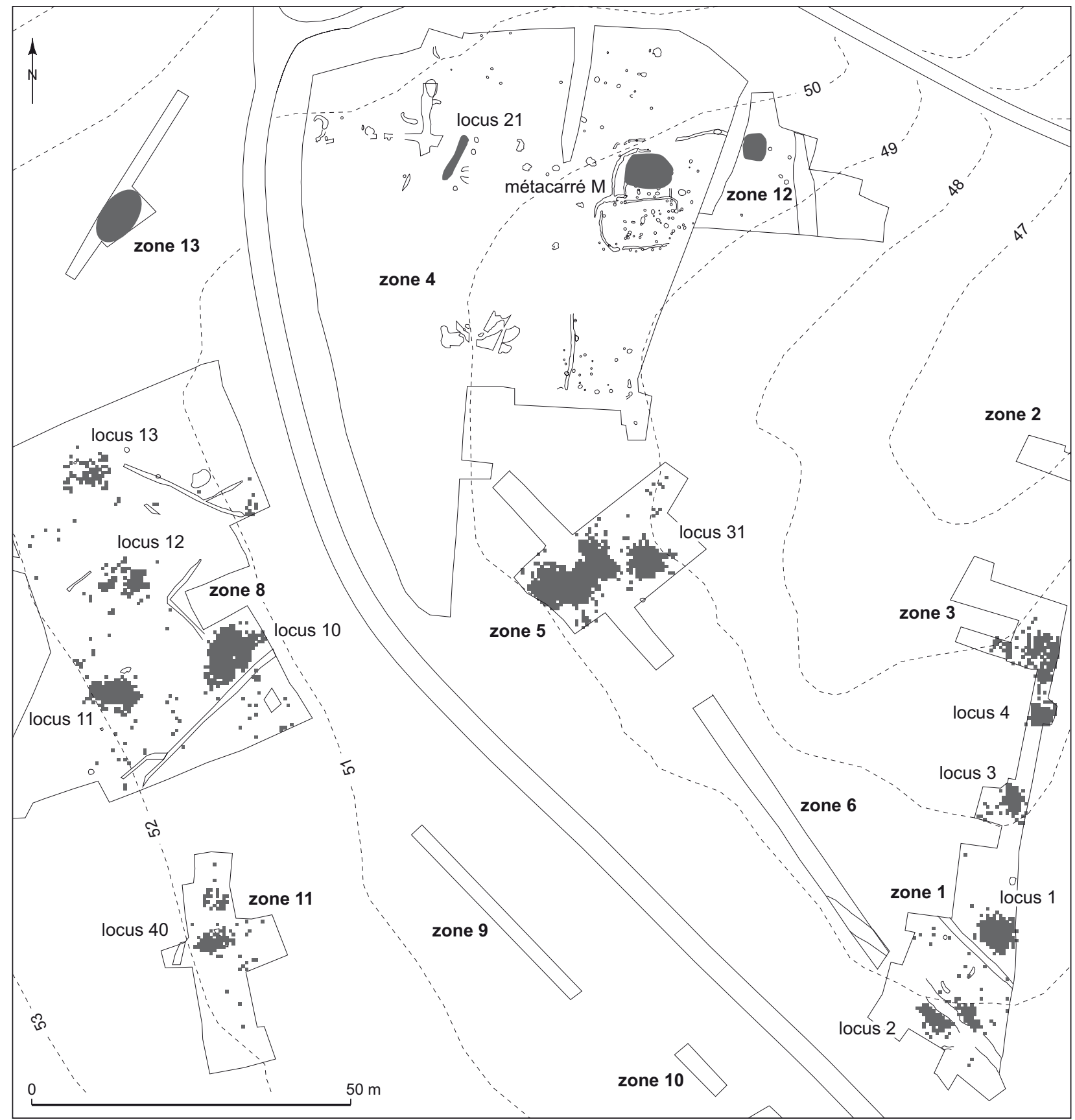

Fig. 14 - Plan général des concentrations de silex (par quart de mètre carré, à l'exception des zones 4 et 12 où sont signalées les concentrations principales). Les principales structures de l'âge du Fer sont également marquées (dessins: S. Sicard, Inrap et J.-F. Nauleau, Inrap).

De Bie, Caspar, 2000). Le matériel situé près de ces pierres n'est pas particulièrement brûlé. Au contraire, le locus 4 fait partie de ceux dont seulement $25 \%$ du matériel est altéré par le feu. Ce petit ensemble de pierres corrobore encore l'interprétation du locus 3 comme aire de rejet, sans aménagement notable. Un autre petit foyer, bien conservé celui-là, gisait plus au nord en zone 3, hors d'un locus. Il s'agit justement d'un secteur altéré par des troncatures sédimentaires et un charbon prélevé entre les pierres a donné une date du Néolithique moyen: $5225 \pm 60 \mathrm{BP}$, soit entre 4222 et 3945 avant notre ère (Lyon-1073-OXA). Cette absence de structures de combustion empierrées est courante en 
Tabl. II - Inventaire des zones ayant livré du silex.

\begin{tabular}{|c|c|c|c|c|c|c|c|c|c|}
\hline Zone & Locus & Divers & Bouleversement & Fouille & Homogénéité & Tamisage & N (relevé) & $\mathbf{N}$ (tamis) & Datation \\
\hline \multirow{2}{*}{1} & 1 & - & deux ornières anciennes & totale & oui & total & 819 & 2226 & Azilien \\
\hline & 2 & - & fossé romain & totale & oui & aucun & 364 & 1504 & Azilien \\
\hline \multirow{3}{*}{3} & 3 & - & aucun & totale & oui & aucun & 168 & 49 & Azilien \\
\hline & 4 & - & - & partielle & oui & aucun & 281 & 146 & Azilien \\
\hline & - & hors & troncature sédimentaire & partielle & non & aucun & 291 & - & Azilien \\
\hline \multirow{2}{*}{4} & - & 20 & - & partielle & non & aucun & 527 & - & divers \\
\hline & 21 & - & tranchée de prospection & totale & oui & aucun & 157 & - & Azilien \\
\hline \multirow{2}{*}{5} & - & - & tranchée d'évaluation & partielle & $?$ & aucun & 2258 & - & Azilien \\
\hline & 31 & - & aucun & partielle & $?$ & aucun & 819 & - & Néolithique final \\
\hline 7 & - & - & - & partielle & non & aucun & - & - & - \\
\hline \multirow{4}{*}{8} & 10 & - & décapage partiel & partielle & oui & aucun & 1109 & 1118 & Azilien \\
\hline & 11 & - & $\begin{array}{l}\text { tranchée d'évaluation } \\
\text { décapage de la fouille }\end{array}$ & totale & oui & partiel & 1209 & - & Azilien \\
\hline & 12 & - & décapage de la fouille & totale & oui & total & 354 & 339 & Azilien \\
\hline & 13 & - & aucun & totale & oui & total & 248 & 357 & Azilien \\
\hline 11 & 40 & - & décapage partiel & partielle & oui & aucun & 345 & - & Azilien \\
\hline 12 & - & - & rare & partielle & oui & aucun & 565 & - & Azilien \\
\hline 13 & - & - & décapage partiel & partielle & oui & aucun & 322 & - & Azilien \\
\hline
\end{tabular}

contexte d'habitat azilien récent. Aux Chaloignes, les soles ont-elles été systématiquement lessivées, étant donné leur faible enfouissement? Des foyers existent-ils dans des zones non fouillées du site? Cette dernière possibilité semble bien restreinte; en revanche les bioturbations et l'hydromorphie sont peut-être venues à bout des traces de feu sur le sol. Dans tous les cas, il est difficile d'admettre qu'il n'y ait pas eu de foyers en fonctionnement au moment des occupations aziliennes, et l'on privilégiera l'hypothèse de foyers à plat, à proximité des locus.

Les analyses de l'industrie lithique ont pris appuis sur cette distinction. La description générale de l'industrie lithique se fera uniquement à partir des locus homogènes $(1,2,3,4,11,12,13$ et 40) et à l'exclusion de la zone 13, certes homogène, mais prélevée trop rapidement. Pour des commodités de lecture des figures, le relevé des pièces en trois dimensions a souvent été basculé en décompte par quart de mètre carré. Plusieurs semaines ont été consacrées aux remontages au sein des locus, qui ont permis d'affiner la compréhension de la dynamique du débitage ou de percevoir des faciès proches parmi les matières. La fraction lithique recueillie au tamisage n'est jamais incluse dans les statistiques, afin de permettre les comparaisons sur les mêmes bases entre les locus. Leur apport est cependant intégré dans la compréhension technique de chaque locus.

\section{IDENTIFICATION DES MATIÈRES PREMIÈRES ET APPROCHE DES TERRITOIRES D'ACQUISITION}

\section{COMPRENDRE UN CIRCUIT ÉCONOMIQUE SUR LES MARGES DU MASSIF ARMORICAIN}

Installés sur un massif ancien dépourvu de formations sédimentaires à silex mais à proximité du Bassin parisien, les hommes préhistoriques des Chaloignes ont développé une économie particulière d'acquisition des matériaux, une signature culturelle qu'il importe de révéler. L'acquisition privilégie clairement une récolte au plus près, ici dans les terrasses de la Loire, mais l'importation de matières siliceuses à partir des marges sédimentaires du Massif armoricain est un comportement également attesté. Le fait que l'acquisition à courte distance soit prioritaire a un sens en terme d'organisation économique et sociale; il est également important pour qualifier le degré de flexibilité des normes techniques. Il convient donc, pour chacune de ces matières, d'en chercher l'origine, mais également de définir la morphologie initiale des blocs, leur qualité à la taille ou le degré d'avancement du débitage à l'arrivée sur le locus. Ces motions de principe se heurtent ici à la grande diversité des origines de silex roulés par la Loire, puisque ce fleuve draine toute la France moyenne et traverse nombre de niveaux sédimentaires jurassiques et 
crétacés. Il en résulte une très grande variété des galets roulés, plus manifeste d'ailleurs qu'à l'embouchure du fleuve, sans que nous ne puissions proposer d'explication. Si l'on ne tient pas compte du transport par la Loire, le risque évident est de surévaluer la part des matières importées par les hommes: un silex a pu en effet être à la fois récolté à longues distances sur les affleurements et à proximité dans les terrasses. Pour s'adapter à la proximité de ce grand «collecteur» fluvial, notre classement a donc suivi une voie originale, avec une première grille à trois entrées : «cortex roulé », "cortex non-roulé » et «sans cortex». Une seconde grille a été appliquée exclusivement aux matières à cortex non-roulé, avec plusieurs types définis par une lettre. La tendance a été à une forte séparation des faciès, que l'on a pu regrouper ensuite. La catégorie non-roulé intègre des matières trop rares et dont les caractères peu prononcés auraient rendu la description inopérante. À cause de cette méthode, la part des matières importées depuis les marges sédimentaires du Massif armoricain risque d'être un peu sous-évaluée, si l'on considère qu'un décorticage partiel a pu se faire hors des locus (pour ne pas dire hors du site) ou encore que ces blocs plus volumineux que des galets roulés exhibent moins de surfaces corticales par rapport à leur volume de matière. Mais les tests effectués sur certains locus ne montrent pas d'écarts supérieurs à $2 \%$. L'estimation de la morphologie du bloc à son arrivée sur le locus pâtit aussi de ce mode d'analyse des matériaux, puisque les pièces non-corticales vont rejoindre la catégorie indéterminée qui connaît alors une forte expansion. Il convient donc à chaque fois de compléter le diagnostic lors de la phase de remontage des pièces.

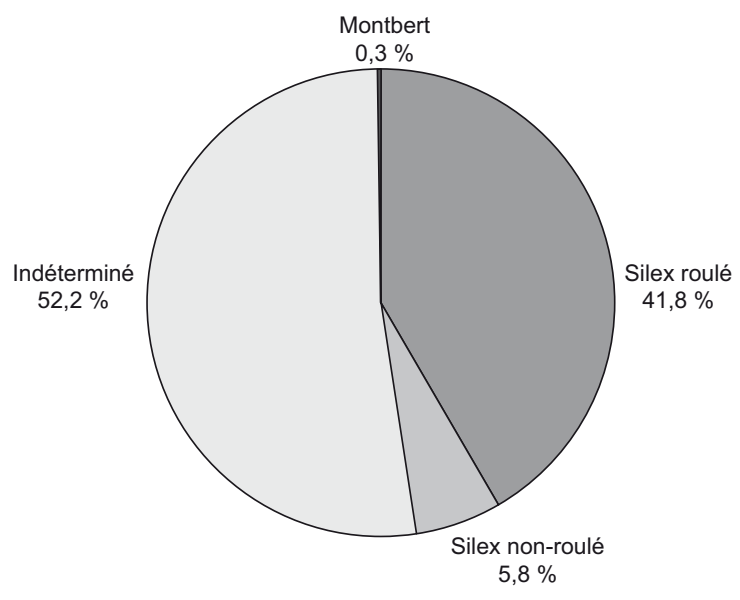

Fig. 15 - Locus homogènes (1, 2, 3, 4, 11, 12, 13, 40). Répartition des matières premières (avec indéterminés, soit 3786 pièces).
La seconde partie de l'exercice consiste à rechercher les formations originelles des matières siliceuses à cortex non-roulé. Sans l'aide de géologues de terrain (M. Gruet, G. Moguedet, D. Poncet), ces travaux préliminaires auraient été vains. Nous avons complété ces indications par des visites sur des affleurements les plus proches signalés par ces chercheurs, entre Angers, Saumur et Doué-la-Fontaine (Maine-et-Loire). Il s'agit d'une zone de contact entre le Massif armoricain et le Bassin parisien avec, d'une part une remontée des couches géologiques anciennes sur le massif, d'autre part un jeu sédimentaire et érosif complexe lié aux transgressions marines sur des marges successivement inondées et exondées. De ces mouvements littoraux résulte une prépondérance des dépôts sableux ou marneux. Des silex d'excellente qualité sont malgré tout présents dans des niveaux crétacés (tuffeau du Turonien à $20 \mathrm{~km}$ au minimum des Chaloignes, calcaire lacustre du Bartonien à $25 \mathrm{~km}$ au minimum) ou jurassique (Bajocien à $18 \mathrm{~km}$ au minimum).

\section{LES MATIÈRES TAILLÉES, LEUR ORIGINE}

Si l'on ne tient compte que des matières déterminées par leur cortex, les silex importés représentent $14,7 \%$ du matériel et il y a moins de $1 \%$ de quartzite de Montbert (tabl. III et IV; fig. 15 et 16). Les matières C, E, F et L sont présentes en quantités trop anecdotiques pour être définies; d'autres matières sont en revanche plus aisées à décrire et à analyser.

Les silex roulés proviennent, comme sur le littoral armoricain, des plages de galets, la Loire remplaçant ici l'océan. Si la composante blonde translucide à grain très fin domine

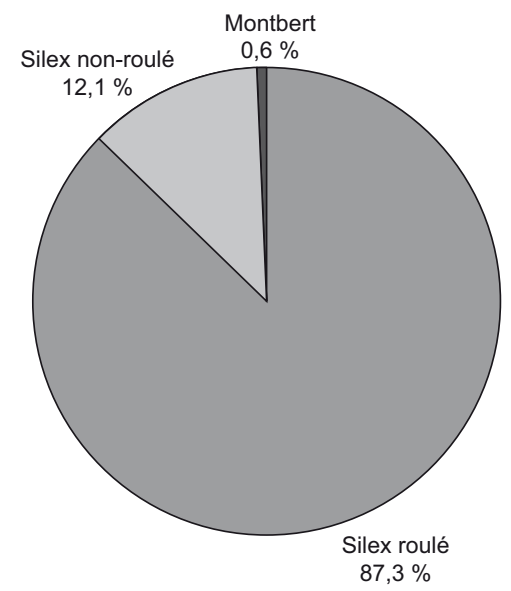

Fig. 16 - Locus homogènes (1, 2, 3, 4, 11, 12, 13, 40). Répartition des matières premières (hors indéterminés, soit 1811 pièces). 
Tabl. III - Description des principales matières identifiées sur le site et représentation proportionnelle des matières (hors indéterminées).

\begin{tabular}{|c|c|c|}
\hline Matière & Description & $\%$ \\
\hline B & $\begin{array}{l}\text { - Silex noir ou brun foncé, avec des zones plus claires (oranges et beiges) et des inclusions blondes millimétriques, translucide, très fin, homogène } \\
\text { - Cortex blanc beige crayeux épais }(+5 \mathrm{~mm})\end{array}$ & 0,7 \\
\hline C & - Silex blanc opaque mat, à vacuoles et plages jaunes translucides & 0,0 \\
\hline D & $\begin{array}{l}\text { - Silex blond translucide à taches blanches millimétriques, très légèrement grenu avec un effet de paillettes } \\
\text { - Cortex crayeux blanc peu épais, non roulé }\end{array}$ & 0,9 \\
\hline $\mathbf{E}$ & $\begin{array}{l}\text { - Silex gris-brun clair, à bandes noires, opaque, très homogène } \\
\text { - Cortex inconnu }\end{array}$ & 0,5 \\
\hline $\mathbf{F}$ & $\begin{array}{l}\text { - Silex blanc (d'aspect quartz), opaque mat, grenu, de médiocre qualité } \\
\text { - Cortex inconnu }\end{array}$ & 0,1 \\
\hline G & $\begin{array}{l}\text { - Silex noir, opaque, mat } \\
\text { - Cortex brun clair à zone sub-corticale très épaisse }(10 \text { à } 20 \mathrm{~mm}) \text {, non roulé }\end{array}$ & 3,4 \\
\hline $\mathbf{H}$ & $\begin{array}{l}\text { - Silex beige-brun clair, à tâches blanches allongées, opaque, mat, de qualité moyenne } \\
\text { - Cortex blanc beige peu épais }(1-2 \mathrm{~mm}) \text {, zone sub-corticale en silex blanc }(5 \mathrm{~mm})\end{array}$ & 0,7 \\
\hline I & $\begin{array}{l}\text { - Jaspe marron, avec de minces rubans blanchâtres sinusoïdaux (moins d'1 mm), brillant, totalement opaque } \\
\text { - Cortex infra-millimétrique beige, poudreux, hydrophile (collant à la langue), sans zone sub-corticale, non roulé }\end{array}$ & 0,2 \\
\hline $\mathbf{J}$ & $\begin{array}{l}\text { - Silex marron à micro paillettes, avec beaucoup d'inclusions corticales } \\
\text { - Cortex épais, non roulé }\end{array}$ & 0,8 \\
\hline $\mathrm{K}$ & $\begin{array}{l}\text { - Silex à dominante brun clair, à tâches multiples marrons ou blanches, à nombreux squelettes de fossiles } \\
\text { - Cortex très peu épais (1-2 mm), compact, homogène, non roulé }\end{array}$ & 0,8 \\
\hline $\mathbf{L}$ & $\begin{array}{l}\text { - Silex marron à larges rubans gris ou beiges et à nombreuses ponctuations blanches } \\
\text { - Silex non roulé à cortex épais d'aspect poreux, avec un liseré noir en guise de zone sub-corticale }\end{array}$ & 0,0 \\
\hline $\mathbf{M}$ & $\begin{array}{l}\text { - Silcrète beige-orange veiné de brun. Présence d'inclusions crayeuses } \\
\text { - Cortex épais pulvérulent, crayeux, d'aspect feuilleté. Présentation en plaquette de } 30 \text { à } 50 \mathrm{~mm} \text { d'épaisseur }\end{array}$ & 3,0 \\
\hline $\mathbf{N}$ & $\begin{array}{l}\text { - Silex marron, opaque, légèrement grenu, bandes rougeâtres aux contours mal définis } \\
\text { - Cortex épais }(10-15 \mathrm{~mm})\end{array}$ & \\
\hline
\end{tabular}

Tabl. IV - Répartition générale des matières taillées dans les principaux locus (avec indéterminés).

\begin{tabular}{|l|r|r|r|r|r|r|r|r|r|r|}
\hline Matière / locus & \multicolumn{1}{|c|}{} & \multicolumn{1}{|c|}{$\mathbf{2}$} & $\mathbf{3}$ & $\mathbf{4}$ & $\mathbf{1 0}$ & $\mathbf{1 1}$ & $\mathbf{1 2}$ & $\mathbf{1 3}$ & $\mathbf{4 0}$ & Total \\
\hline B & 0,9 & 0,0 & 0,0 & 0,4 & 0,1 & 0,2 & 0,8 & 0,0 & 0,0 & 0,3 \\
\hline C & 0,1 & 0,0 & 0,0 & 0,0 & 0,0 & 0,0 & 0,0 & 0,0 & 0,0 & 0,0 \\
\hline D & 0,7 & 0,3 & 0,0 & 1,0 & 0,5 & 0,3 & 0,3 & 0,0 & 0,3 & 0,4 \\
\hline E & 1,0 & 0,0 & 0,0 & 0,0 & 0,0 & 0,2 & 0,3 & 0,0 & 0,0 & 0,2 \\
\hline F & 0,0 & 0,3 & 0,0 & 0,0 & 0,1 & 0,1 & 0,0 & 0,0 & 0,0 & 0,1 \\
\hline G & 0,0 & 0,0 & 0,0 & 0,0 & 5,9 & 0,9 & 0,0 & 0,0 & 0,3 & 1,6 \\
\hline H & 0,0 & 0,0 & 0,0 & 0,0 & 0,6 & 0,7 & 0,0 & 0,0 & 0,0 & 0,3 \\
\hline I & 0,0 & 0,0 & 0,0 & 1,4 & 0,0 & 0,1 & 0,0 & 0,0 & 0,0 & 0,1 \\
\hline Indéterminé & 47,7 & 50,8 & 61,8 & 46,5 & 53,0 & 56,6 & 50,8 & 43,8 & 55,9 & 52,3 \\
\hline J & 0,0 & 0,0 & 0,0 & 0,0 & 1,4 & 0,1 & 0,3 & 0,0 & 0,3 & 0,4 \\
\hline K & 0,0 & 0,0 & 0,6 & 0,0 & 1,6 & 0,0 & 0,0 & 0,0 & 0,0 & 0,4 \\
\hline L & 0,0 & 0,0 & 0,0 & 0,0 & 0,0 & 0,0 & 0,0 & 0,0 & 0,3 & 0,0 \\
\hline M & 0,0 & 0,0 & 0,0 & 27,7 & 0,0 & 0,0 & 0,0 & 0,0 & 0,0 & 1,6 \\
\hline Montbert & 0,2 & 0,3 & 0,0 & 0,0 & 0,1 & 0,3 & 0,8 & 0,0 & 0,0 & 0,2 \\
\hline Silex non roulé & 2,6 & 1,1 & 0,0 & 2,1 & 1,3 & 1,1 & 4,2 & 0,8 & 1,2 & 1,6 \\
\hline Silex roulé & 46,8 & 47,0 & 37,6 & 20,9 & 35,3 & 39,3 & 42,4 & 55,4 & 41,7 & 40,3 \\
\hline Total (en \%) & $\mathbf{1 0 0 , 0}$ & $\mathbf{1 0 0 , 0}$ & $\mathbf{1 0 0 , 0}$ & $\mathbf{1 0 0 , 0}$ & $\mathbf{1 0 0 , 0}$ & $\mathbf{1 0 0 , 0}$ & $\mathbf{1 0 0 , 0}$ & $\mathbf{1 0 0 , 0}$ & $\mathbf{1 0 0 , 0}$ & $\mathbf{1 0 0 , 0}$ \\
\hline
\end{tabular}

sans conteste, elle ne représente pas toute la gamme des teintes. Dans le locus 13 notamment, la teinte rouge est majoritaire parmi ces silex roulés, sans que les atteintes du feu ne soient en cause. La récolte a pu se faire sur les berges du fleuve, mais les auteurs de la carte géologique signalent une quinzaine de mètres d'alluvions récentes en aval des Pont-de-Cé (Maine-et-Loire), soit à la hauteur des Chaloignes, ce qui empêche tout contrôle direct. D'anciennes hautes terrasses du fleuve aujourd'hui démantelées auraient pu également être mises à profit, mais aucune preuve ne peut être apportée pour soutenir cette hypothèse. Il est difficile dans ces conditions de reconnaître les lieux précis de récolte.

La matière $\mathrm{M}$ est connue sous le terme de perron, ou perron de Touraine. Il s'agit d'une silicification de matériaux argileux (horizon B argillique d'un sol formé sur substrat sableux -Mouyoungou, 1990). Ces silcrètes d'âge éocène (de l'Yprésien au Bartonien) sont fréquents en Anjou oriental, où on les trouve souvent en entablements massifs. Ils semblent rares à proximité du Massif armoricain, où la silicification s'est plutôt faite aux dépens de matériaux sableux. Il reste à localiser le gisement d'origine de ce silcrète si particulier, mais il semble inconnu à moins de $30 \mathrm{~km}$ vers l'est. Selon $\mathrm{D}$. Poncet, la matière $\mathrm{M}$ est inconnue dans le Saumurois ou dans le seuil du Poitou.

La matière $G$ est un silex assez répandu dans les sites préhistoriques de la région reconnus en surface. La qualité de la roche et la forme allongée ou branchue de certains blocs laissent penser à une silicification du Crétacé (Turonien), courante dans le tuffeau. Sous toutes réserves, 


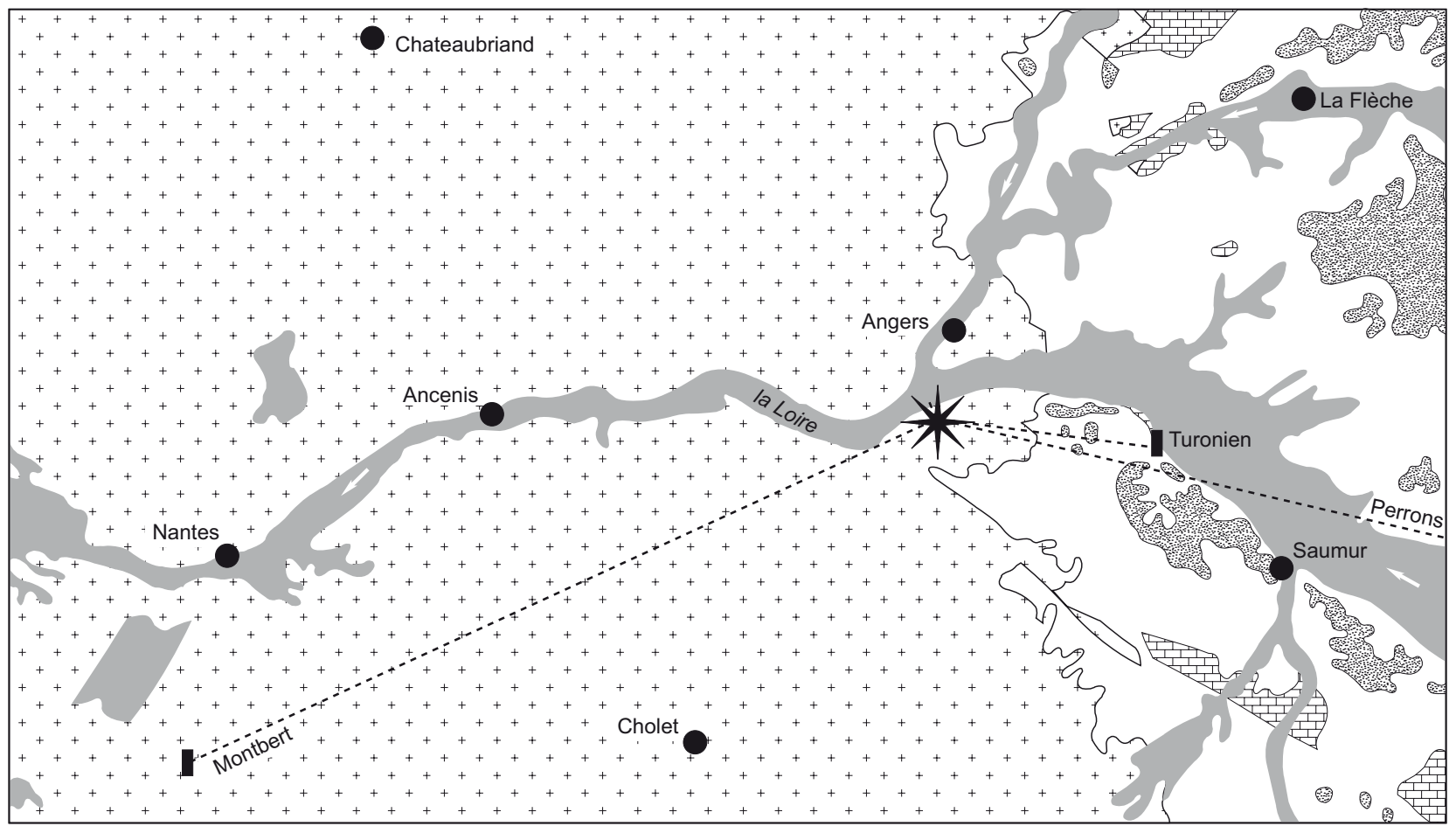

0 75 150

kilomètres

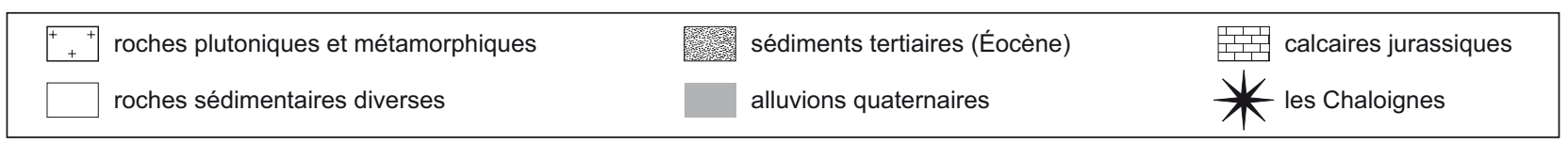

Fig. 17 - Carte géologique simplifiée. Origine des silex G (probablement turoniens) et des quartzites de Montbert. Les silcrètes de type M (ou perrons) viendraient de Touraine, sans précision supplémentaire (dessin: G. Marchand, CNRS UMR 6566).

les types $\mathrm{J}$ et E pourraient y être assimilés, ainsi que le silex $\mathrm{N}$ de la zone 5, dans des variantes plus claires. La présence de muscovite dans ce dernier confirmerait d'ailleurs cette attribution. L'origine exacte de ces silex noirs est délicate à apprécier sans un long travail de terrain, puisque le tuffeau d'Anjou est présent à $10 \mathrm{~km}$ à l'est des Chaloignes. Toutefois, selon la carte géologique, les silex n'apparaîtraient que $10 \mathrm{~km}$ plus loin vers l'est (fig. 17).

La matière I est un jaspe orange uni, apparemment importé sous forme de supports de plein débitage (locus 4 dans la zone 3). On peut rencontrer des faciès jaspoïdes dans les calcaires lacustres du Bartonien, par exemple à Pompierre (Chênehutte-les-Tuffeaux, Maine-et-Loire), à $30 \mathrm{~km}$ au sud-est des Chaloignes. Les faciès échantillonnés sur le gisement géologique semblent cependant nettement moins fins que sur le site préhistorique.
Les matières $\mathrm{D}$ et $\mathrm{K}$, pourtant assez abondantes, ne trouvent pas encore de parallèles dans la région. La seconde correspondrait à des blocs de grandes dimensions, très branchus. Les autres matières sont trop rares pour que l'on ose proposer des parallèles.

Le quartzite de Montbert (Loire-Atlantique), grès tertiaire aux remarquables aptitudes à la taille, est connu sur une seule source actuellement, au sud de Nantes, à $75 \mathrm{~km}$ des Chaloignes. La découverte de gîtes plus proches reste possible, tant ces dépôts tertiaires sont nombreux à l'est du Massif armoricain, mais aujourd'hui seule la Loire-Atlantique nous livre ce quartzite fin très particulier (dit aussi quartzarénite). Nous adopterons donc la position de G. Gouraud et M. Le Goffic, qui considèrent ce gisement comme unique tant que d'autres sources n'auront pas été définies (Gouraud, Le Goffic, 1998). Il convient de noter que M. Gruet, fin 
connaisseur de la géologie régionale, donnait aussi Montbert comme origine pour des quartzites identiques trouvés dans les niveaux paléolithiques de Roc-en-Pail (Chalonnes-surLoire, Maine-et-Loire), à proximité des Chaloignes (Gruet, 1990). Les qualités à la taille de cette matière grenue étant moindres que celles du silex, il convient d'insister sur un transport qui ne répond pas à une stricte logique technique ou fonctionnelle et qui révèle un axe de déplacement vers l'ouest, peut-être suivant le couloir ligérien.

La rapide enquête menée sur le terrain nous permet surtout de signaler des absences. Entre Saint-Rémy-laVarenne et Le Thoureil (Maine-et-Loire), la falaise entaillant les niveaux du Jurassique moyen (Bajocien) livre beaucoup de silex échantillonnés dans le cadre du présent travail, que l'on ne retrouve pas dans le matériel des Chaloignes. De même, les silex et meulières des calcaires lacustres d'Anjou (Bartonien) n'ont pas été rencontrés, à l'exception peut-être de la matière I, alors qu'ils sont particulièrement abondants entre Gennes et Saumur (Maine-et-Loire), en affleurements et surtout en surface dans des argiles à silex. Dans les deux cas, il aurait fallu parcourir une vingtaine de kilomètres à vol d'oiseau pour s'approprier ces matériaux disponibles aisément, ce qui n'a pas été réalisé par les occupants des Chaloignes. On pense évidemment à des problèmes d'accessibilité et notamment à un empâtement de l'actuelle falaise de la Loire (pour le premier) ou des plateaux (pour le second). Beaucoup reste à faire pour déterminer l'origine des matériaux taillés aux Chaloignes, mais deux points sont d'ores et déjà importants à retenir pour la suite de cette étude. En premier lieu, l'acquisition sur les sources les plus proches, probablement à moins de $5 \mathrm{~km}$, a été la solution la plus couramment adoptée par les hommes du Paléolithique final. En second lieu, des acquisitions à moyennes distances (1 à 3 jours de marche) apparaissent sporadiquement. Elle se ferait suivant l'axe ligérien, soit vers la Touraine, soit plus rarement vers la région nantaise, avec des distances variant de 30 à $75 \mathrm{~km}$ (fig. 17). Il n’y a pas de silex de la région de Thouars (Deux-Sèvres), aux gisements de silex secondaires pourtant si abondants. Le fait que des matériaux intermédiaires soient négligés mériterait d'être confirmé, puis expliqué. Il faut donc conclure que les galets de silex des terrasses de la Loire suffisent à la réalisation du système technique azilien. Les analyses des pages suivantes s'attacheront à en comprendre la logique, mais on peut dès à présent remarquer que ces galets ovoïdes offrent suffisamment de convexités pour entamer un débitage de produits peu standardisés, sans grand effort de mise en forme. Cette qualité doit cependant être relativisée par un fort taux de pièces gélives et faillées, qui ont très souvent compromis le débitage. Enfin, les silex disponibles à l'affleurement dans un rayon de $30 \mathrm{~km}$ sont gris et opaques, tandis que les galets de silex charriés par la Loire sont jaunes et translucides, parfois luisants. Ce paramètre eut-il un sens dans le système de valeur azilien? Seules des études d'autres collections permettraient de le démontrer.

G. M. et S. S.

\section{ORIGINE DES CRAYONS D'OCRE}

Deux fragments de crayons d'ocre ont été découverts sur le site, l'un dans le locus 20 de la zone 4 (fig. 18), l'autre dans le locus 11 de la zone 8 . Les analyses ont été effectuées par diffraction des rayons X à l'UMR 6566 à l'université de Rennes-I. Une première analyse a été faite en plaçant directement les deux crayons dans le diffractomètre Rigaku Miniflex de l'UMR 6566. Cette méthode ne s'étant pas révélée satisfaisante, une seconde analyse a été faite sur l'échantillon L 386 (zone 8, locus 11, no 1162), présumant que les deux crayons étaient de même nature; un prélèvement de quelques milligrammes a été effectué. L'acquisition a été faite avec un tube à anticathode au cuivre, sur un large domaine angulaire, de $4^{\circ}$ à $71^{\circ}$, avec un pas de $0,02^{\circ}$ et un temps de comptage de 20 secondes. La durée de l'acquisition a été d'environ 19 heures. Une fois le diagramme des poudres obtenu, un dépouillement a été réalisé sur le spectre de diffraction à rayons $\mathrm{X}$, en utilisant le logiciel Eva et la base de données JCPDS.

Le dépouillement du diagramme des poudres met en évidence la présence de trois minéraux:

- quartz $\left(\mathrm{SiO}_{2}\right)$ : ce minéral réagit très bien à la diffraction des rayons $\mathrm{X}$, même en petite proportion, ainsi l'importance de l'intensité des raies du quartz ne montre pas forcément une forte présence de celui-ci;

- hématite $\left(\mathrm{Fe}_{2} \mathrm{O}_{3}\right)$ : oxyde de fer de couleur rouge ocre, ce qui correspond à la couleur des crayons;

- illite $\left(\mathrm{K}, \mathrm{H}_{3} \mathrm{O}\right)(\mathrm{Al}, \mathrm{Mg}, \mathrm{Fe}) 2(\mathrm{Si}, \mathrm{Al}) 4 \mathrm{O} 10[(\mathrm{OH}) 2,(\mathrm{H} 2 \mathrm{O})]$ : minéral argileux.

Il s'agit donc bien d'ocre particulièrement riche en hématite. Les formations géologiques originelles sont probablement à chercher dans les formations de grès armoricain, et notamment dans ses formations inférieures. Les zones les plus proches se situent sous la ville d'Angers, à $9 \mathrm{~km} \mathrm{du}$ site des Chaloignes; des formations particulièrement riches en fer sont aussi reconnues à $3 \mathrm{~km}$ au nord, sur toute la lisière du Massif armoricain.

G. Q. et S. L. 


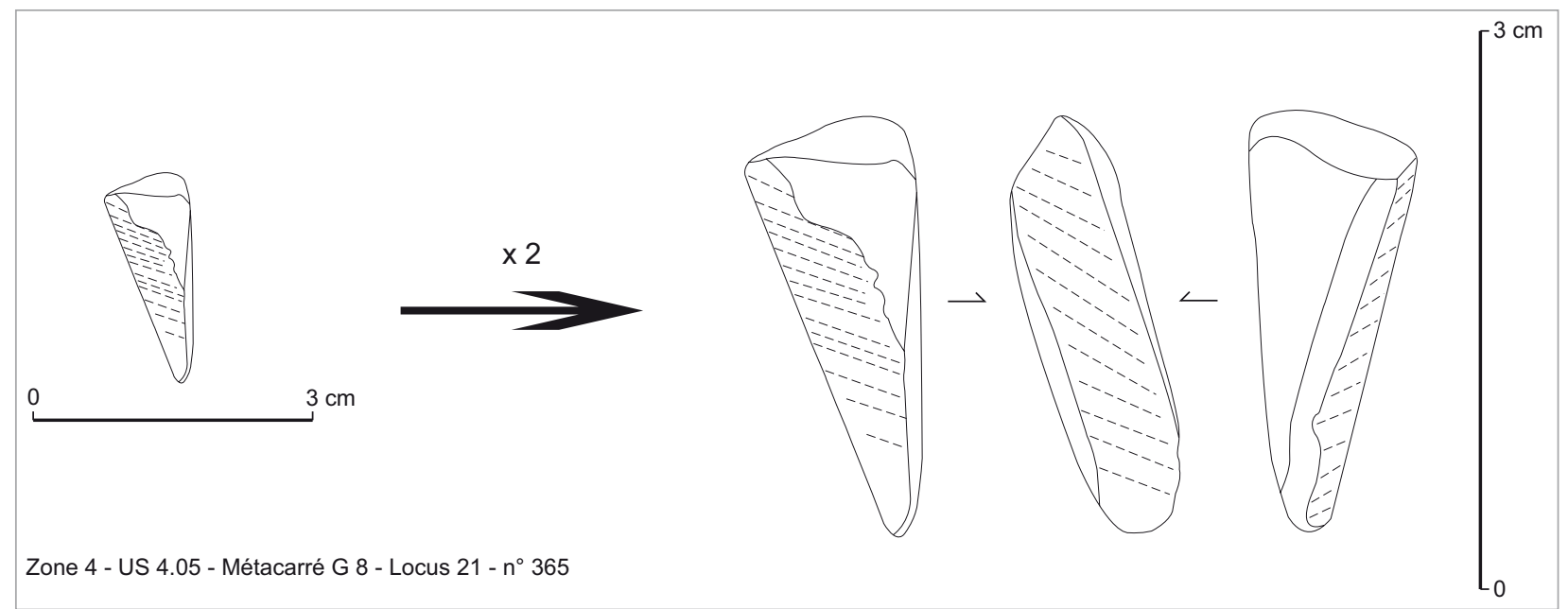

Fig. 18 - Locus 21. Crayon d'ocre (agrandi deux fois), à trois facettes usées (dessin: G. Marchand, CNRS UMR 6566).

\section{LES FLUX DE MATIÈRES}

Comment la matière taillée a-t-elle été importée sur le site? Il importe de poser cette question pour chaque locus, puisque l'on considère au préalable chacune de ces concentrations comme unique. Une des premières observations concerne la forte dispersion des matières sur l'habitat azilien. En règle générale, toutes les phases des chaînes opératoires de débitage sont représentées pour les galets roulés; il n'en reste pas moins que le débitage sur place ne peut être affirmé pour chaque bloc. Des silex à cortex non-roulé peuvent également montrer des séquences de débitage poussées - mais jamais complètes - ou alors être représentées par des pièces isolées: ainsi un nucléus en silex $\mathrm{G}$ dans le locus 40 ou une lamelle en matière $\mathrm{M}$ en zone 12. Cette matière $\mathrm{M}$, si particulière, est pour l'essentiel taillée dans le locus 4, à l'est du site. Mais elle est également connue au sud du site, en zone 8 (dans un sondage de l'évaluation), ou au nord en zone 4 (locus 21). Le préhistorien actuel est évidemment tenté de considérer qu'une matière aussi rare n'a été ramassée qu'une fois, assertion éventuellement corroborée par une accumulation de déchets de taille à un unique endroit. Au-delà d'un comportement anecdotique, cela signifierait une contemporanéité du locus 4 , de la zone 12, du locus 21 et d'un emplacement mal défini en zone 8, soit la quasi-totalité de la cuvette des Chaloignes. Mais l'aspect visuel particulier de cette roche aurait pu tout aussi bien lui conférer un statut économique particulier; puisqu'elle attire l'attention, elle aurait même pu être dispersée sur l'habitat à chaque nouvelle occupation au gré des accès de curiosité.
Le quartzite de Montbert est une autre roche très caractéristique et donc emblématique de la dissémination des chaînes opératoires dans l'espace: il y a des outils, ce qui est logique pour une fin de chaîne, mais on note aussi un nucléus en zone 5 ou une néo-crête dans le locus 10, tous deux isolés. Certaines autres matières non-roulées semblent avoir été importées sous forme de grands supports laminaires, alors que d'autres comme le silex G ont fait l'objet d'un débitage exhaustif. Les locus des Chaloignes sont des zones à l'évidence ouvertes, sans que l'on puisse préciser si elles échangent exclusivement avec l'extérieur de l'habitat (il s'agirait alors de témoins d'occupations régulières par des petites unités sociales) ou avec l'intérieur (plusieurs locus fonctionneraient ensemble, signalant un groupe plus important). Hormis cette ambiguïté chronologique, la diversité des matériaux sur des aires restreintes et leur dissémination sont significatives du système technique azilien et de son économie de la matière première.

G. M.

\section{CARACTÈRES TECHNIQUES ET TYPOLOGIQUES DES INDUSTRIES LITHIQUES AZILIENNES}

\section{L'OUTILLAGE}

L'éventail typologique est dans l'ensemble peu ouvert (fig. 19) et comparable d'un locus à l'autre (tabl. V et VI). Les armatures, essentiellement composées de pointes aziliennes, sont les marqueurs chronologiques les plus fiables. Les grattoirs courts sur éclat sont aussi très caractéristiques du techno-complexe azilien. Pour le reste, le cortège des 
Tabl. V - Structure générale des industries aziliennes, homogènes ou hétérogènes.

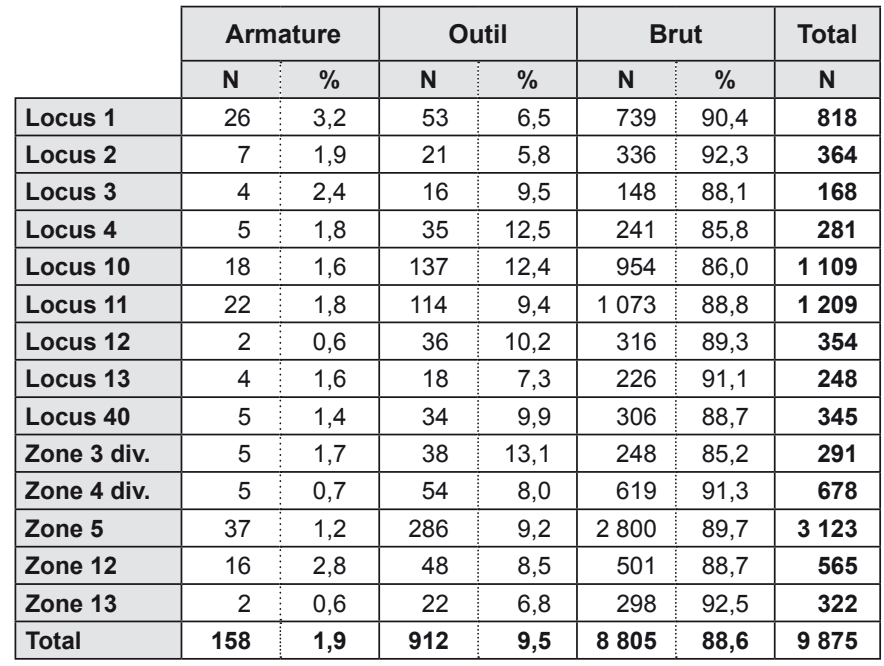

Tabl. VI - Les outils par locus ou zones (hors tamisage).

\begin{tabular}{|c|c|c|c|c|c|c|c|c|c|c|c|c|c|c|c|}
\hline \multirow{2}{*}{$\mathrm{N} /$ Locus } & \multirow{2}{*}{1} & \multirow{2}{*}{2} & \multirow{2}{*}{3} & \multirow{2}{*}{4} & \multirow{2}{*}{10} & \multirow{2}{*}{11} & \multirow{2}{*}{12} & \multirow{2}{*}{13} & \multirow{2}{*}{40} & \multirow{2}{*}{ Zone 3} & \multirow{2}{*}{ Zone 5} & \multirow{2}{*}{ Zone 12} & \multirow{2}{*}{ Zone 13} & \multicolumn{2}{|c|}{ Total } \\
\hline & & & & & & & & & & & & & & $\mathbf{N}$ & $\%$ \\
\hline Pointe azilienne & 16 & 3 & 1 & 2 & 12 & 13 & 1 & 2 & 4 & 1 & 20 & 3 & 2 & 80 & 7,9 \\
\hline Lame étroite à bord abattu & 1 & 0 & 0 & 0 & 1 & 2 & 1 & 0 & 0 & 0 & 2 & 6 & 0 & 13 & 1,3 \\
\hline Pièce à bord abattu & 8 & 4 & 3 & 3 & 2 & 7 & 0 & 2 & 1 & 0 & 13 & 5 & 2 & 50 & 5,0 \\
\hline Segment & 1 & 0 & 0 & 0 & 0 & 0 & 0 & 0 & 0 & 0 & 0 & 0 & 0 & 1 & 0,1 \\
\hline Triangle scalène ou isocèle & 0 & 0 & 0 & 0 & 1 & 0 & 0 & 0 & 0 & 1 & 0 & 1 & 0 & 3 & 0,3 \\
\hline Trapèze & 0 & 0 & 0 & 0 & 2 & 0 & 0 & 0 & 0 & 1 & 0 & 0 & 0 & 3 & 0,3 \\
\hline Armature diverse & 0 & 0 & 0 & 0 & 0 & 0 & 0 & 0 & 0 & 2 & 1 & 1 & 0 & 4 & 0,4 \\
\hline Aileron pédoncule & 0 & 0 & 0 & 0 & 0 & 0 & 0 & 0 & 0 & 0 & 1 & 0 & 0 & 1 & 0,1 \\
\hline Grattoir / éclat & 3 & 2 & 0 & 5 & 11 & 10 & 2 & 1 & 2 & 2 & 29 & 1 & 1 & 69 & 6,8 \\
\hline Grattoir unguiforme & 1 & 0 & 0 & 0 & 0 & 3 & 0 & 0 & 1 & 0 & 0 & 1 & 0 & 6 & 0,6 \\
\hline Grattoir / lame & 3 & 0 & 0 & 0 & 2 & 0 & 0 & 0 & 0 & 0 & 1 & 3 & 0 & 9 & 0,9 \\
\hline Grattoir autre & 0 & 0 & 0 & 0 & 0 & 0 & 1 & 0 & 0 & 0 & 0 & 0 & 0 & 1 & 0,1 \\
\hline Grattoir + burin & 1 & 0 & 0 & 0 & 0 & 2 & 0 & 0 & 0 & 0 & 1 & 0 & 0 & 4 & 0,4 \\
\hline Burin dièdre & 2 & 0 & 0 & 0 & 0 & 0 & 0 & 1 & 1 & 1 & 3 & 2 & 1 & 11 & 1,1 \\
\hline Burin / troncature & 1 & 0 & 2 & 1 & 1 & 1 & 0 & 0 & 6 & 0 & 0 & 2 & 0 & 14 & 1,4 \\
\hline Burin / cassure & 2 & 0 & 2 & 1 & 6 & 5 & 2 & 2 & 2 & 0 & 4 & 2 & 2 & 30 & 3,0 \\
\hline Burin autre & 2 & 0 & 0 & 0 & 0 & 0 & 0 & 0 & 0 & 0 & 0 & 1 & 0 & 3 & 0,3 \\
\hline Éclat à troncature & 1 & 1 & 0 & 1 & 8 & 8 & 1 & 2 & 2 & 0 & 18 & 3 & 2 & 47 & 4,7 \\
\hline Lame à troncature & 0 & 2 & 0 & 1 & 10 & 1 & 1 & 2 & 0 & 0 & 4 & 1 & 2 & 24 & 2,4 \\
\hline Lamelle à troncature & 2 & 1 & 0 & 3 & 4 & 4 & 0 & 0 & 0 & 4 & 6 & 2 & 0 & 26 & 2,6 \\
\hline Éclat à bord abattu & 0 & 0 & 0 & 1 & 10 & 4 & 2 & 0 & 1 & 0 & 13 & 0 & 0 & 31 & 3,1 \\
\hline Lame à bord abattu & 0 & 0 & 1 & 4 & 3 & 9 & 1 & 0 & 0 & 3 & 25 & 2 & 0 & 48 & 4,8 \\
\hline Perçoir & 0 & 0 & 0 & 0 & 0 & 2 & 1 & 0 & 1 & 0 & 2 & 1 & 0 & 7 & 0,7 \\
\hline Éclat denticulé & 0 & 0 & 1 & 1 & 4 & 4 & 0 & 0 & 0 & 1 & 6 & 1 & 0 & 18 & 1,8 \\
\hline Éclat à coche & 0 & 0 & 0 & 1 & 5 & 3 & 1 & 1 & 1 & 2 & 8 & 1 & 1 & 24 & 2,4 \\
\hline Lame à coche & 1 & 0 & 1 & 1 & 0 & 1 & 0 & 1 & 1 & 1 & 2 & 0 & 1 & 10 & 1,0 \\
\hline Lamelle à coche & 0 & 0 & 0 & 0 & 1 & 0 & 0 & 0 & 0 & 0 & 0 & 0 & 0 & 1 & 0,1 \\
\hline Éclat retouché & 15 & 11 & 7 & 8 & 57 & 32 & 17 & 5 & 9 & 16 & 115 & 20 & 5 & 317 & 31,4 \\
\hline Lame retouchée & 13 & 2 & 1 & 5 & 10 & 17 & 7 & 3 & 5 & 8 & 36 & 3 & 3 & 113 & 11,2 \\
\hline Lamelle retouchée & 6 & 2 & 1 & 2 & 5 & 8 & 0 & 0 & 2 & 0 & 12 & 2 & 0 & 40 & 4,0 \\
\hline Poignard & 0 & 0 & 0 & 0 & 0 & 0 & 0 & 0 & 0 & 0 & 1 & 0 & 0 & 1 & 0,1 \\
\hline Total & 79 & 28 & 20 & 40 & 155 & 136 & 38 & 22 & 39 & 43 & 323 & 64 & 22 & 1009 & 100,0 \\
\hline
\end{tabular}




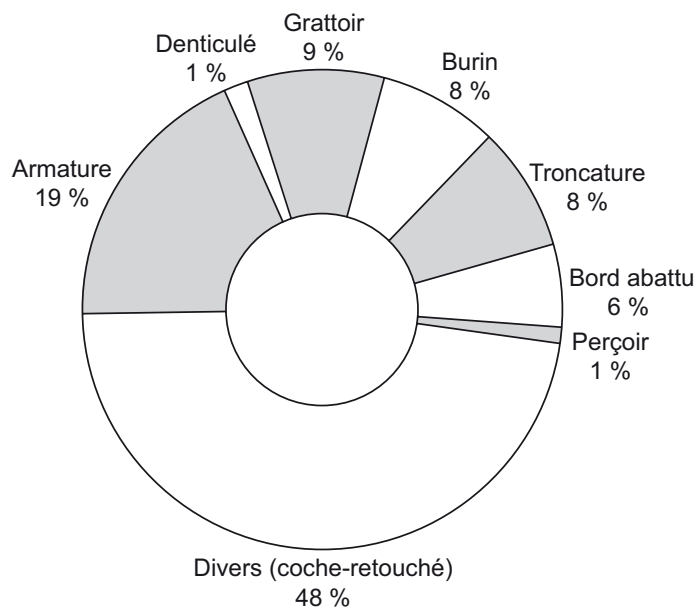

Fig. 19 - Catégories d'outils aménagés ou à fil ébréché sur les locus aziliens homogènes (locus 1, 2, 3, 4, 11, 12, 13, 40).

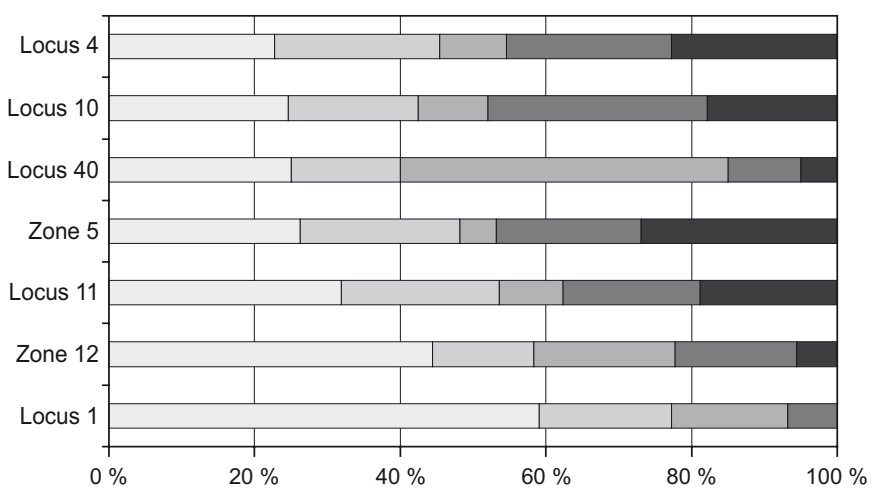

armature $\square$ grattoir $\square$ burin $\square$ troncature $\square$ bord abattu

Fig. 20 - Comparaisons des proportions des principaux outils aménagés (assemblages de plus de 20 pièces). Les taux généraux ne prennent en compte que les locus aziliens homogènes.

outils est peu diversifié, avec une importance similaire des classes de grattoirs, de burins, de troncatures et des bords abattus (fig. 20).

\section{Les armatures: pointes aziliennes et pièces à bord abattu}

Les armatures représentent en moyenne 18,4\% de la totalité de l'outillage. Elles sont réalisées essentiellement sur les lames et lamelles de plein débitage les mieux calibrées, mais quelques éclats plus épais et moins réguliers ont été ponctuellement utilisés.

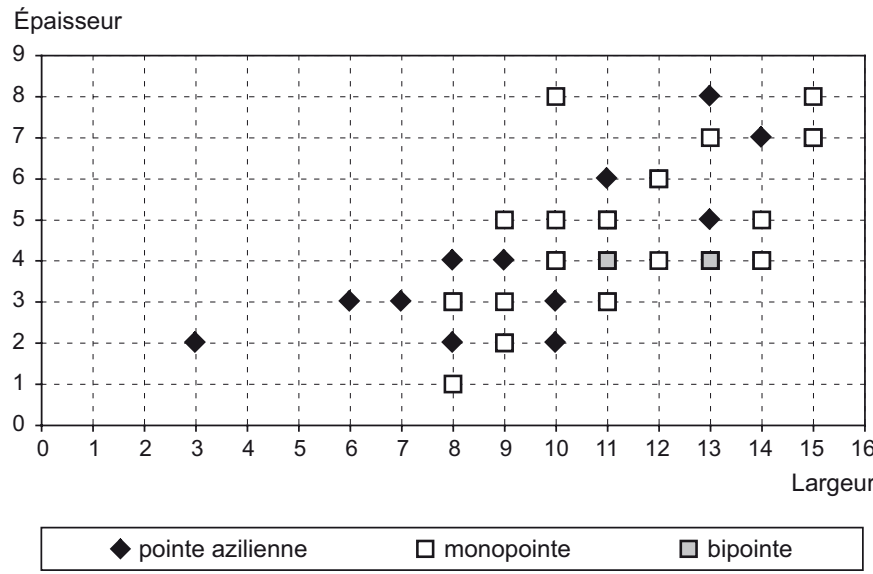

Fig. 21 - Diagramme des largeurs/épaisseurs des pointes aziliennes (en $\mathrm{mm}$ ).

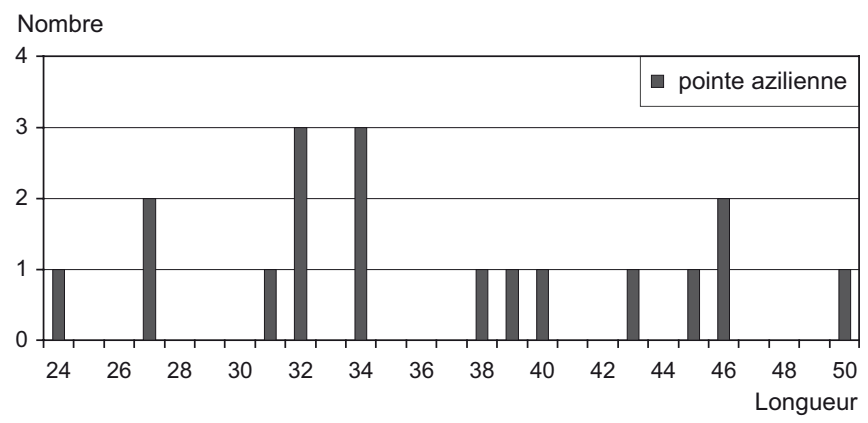

Fig. 22 - Longueur des pointes aziliennes (en $\mathrm{mm}$ ).

Le taux de fragmentation important du matériel a rendu l'attribution typologique des armatures assez ardue; il n'y a que $15,8 \%$ de pièces entières. Nous avons souvent recouru au terme générique de «lame étroite à bord abattu» et encore plus souvent à celui de "pièce à bord abattu", lorsque la pièce était trop partielle pour affiner la lecture. En l'absence d'une base ou des extrémités des pointes, seul le dos permet de reconnaître le type. Le recours systématique au terme de «pointe azilienne» aurait pu gommer l'existence d'ensembles typologiques plus variés. Le terme de pointe a donc été employé seulement lorsque le dos était courbe, même en l'absence de la partie apicale; le caractère convexe du dos est en effet commun à toutes les armatures aziliennes. Il est fort probable que de nombreux fragments de pièce à bord abattu et de pointe azilienne indéterminée décomptés sont des éléments de monopointes. Ainsi, le graphique des largeurs d'armatures montre la complémentarité de ces deux groupes typologiques et donc la très faible ouverture de l'éventail typologique (fig. 21 et 22). 


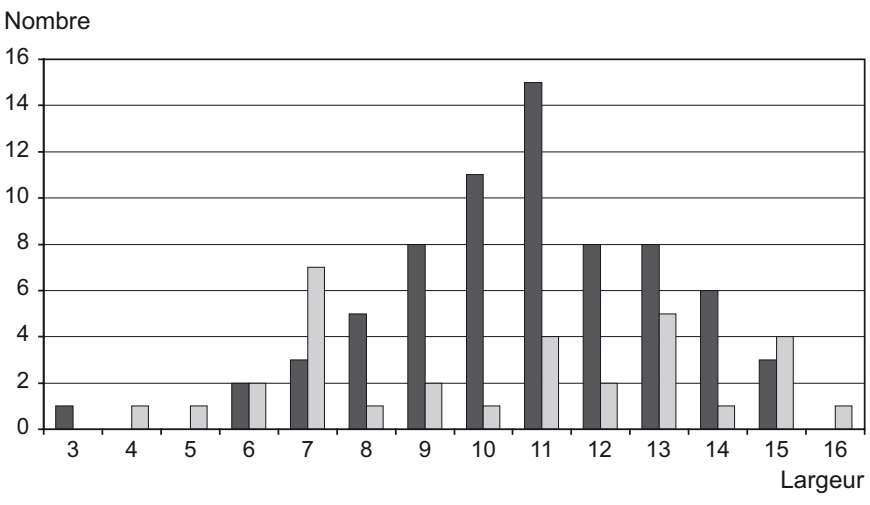

$\square$ pointe azilienne $\quad \square$ pièce à bord abattu

Fig. 23 - Largeurs comparées des pointes aziliennes et des pièces à bord abattu (en $\mathrm{mm})$.

Enfin, lorsque les pièces étaient entières ou lorsqu'une base était visible, nous avons pu utiliser le vocabulaire typologique de manière exhaustive. Ainsi, sur la totalité des locus, nous avons mis en évidence 30 monopointes aziliennes et seulement 3 bipointes. Il y a une grande homogénéité dans le type d'armatures recherché par les occupants du site et la méthode prudente utilisée ici ne doit pas dissimuler l'unité certaine de cette catégorie taxinomique. Le premier élément visuel remarquable est en revanche la faible standardisation de ces armatures: il y a une grande variété dans leur aspect final, et ce malgré la retouche. De ce fait, la distinction entre les pointes aziliennes et les lames à dos est parfois difficile, notamment à partir de fragments.

Les pointes aziliennes peuvent être épaisses ou fines, grandes ou petites et plus ou moins régulières (fig. 24, 35 et 48). Les dimensions des pointes sont variées, mais la largeur et l'épaisseur des pièces sont fortement corrélées; ainsi, plus les pointes sont larges plus elles sont épaisses (fig. 21). Les largeurs sont comprises entre $9 \mathrm{~mm}$ et $13 \mathrm{~mm}$, avec une moyenne à $11 \mathrm{~mm}$. Les épaisseurs varient corrélativement entre $2 \mathrm{~mm}$ et $8 \mathrm{~mm}$, avec une moyenne à $4 \mathrm{~mm}$. Il est intéressant de noter que ces moyennes sont très proches pour les pointes, monopointes aziliennes et pièces à bord abattu (fig. 23). Les largeurs des pièces à bord abattu sont légèrement plus dispersées. On peut supposer que, malgré leur différence typologique, la fabrication de ces pièces procède des mêmes intentions et du même genre d'utilisation. Les longueurs présentent une très forte dispersion; il n'y a jamais de pointe dont la longueur soit inférieure à $25 \mathrm{~mm}$ et le plus fréquemment elle se situe autour de $35 \mathrm{~mm}$, avec un maximum à $50 \mathrm{~mm}$ (fig. 22). Un remontage de 2 fragments de pointe azilienne dans une série laminaire indique une longueur terminale de $43 \mathrm{~mm}$, pour une lame mesurant initialement $4 \mathrm{~mm}$ de plus. Toutefois, ces derniers chiffres ont de faibles valeurs statistiques étant donné le peu de pointes entières.

Dans la morphologie des supports, la rectitude du profil des pièces est un caractère récurrent, alors que les exigences morphométriques sont peu marquées. L'étude du débitage mettra en évidence les moyens techniques mis en œuvre pour la maîtrise et l'entretien de ce type de morphologie. Le caractère typologique commun à toutes ces pointes est le dos, toujours courbe, jamais anguleux. Il est façonné le plus souvent par des retouches croisées, plus rarement par retouches unipolaires. La latéralisation se fait préférentiellement à gauche $(45,3 \%)$, mais le pourcentage d'indéterminé (27\%) vient modérer ce résultat. La base des monopointes est constituée généralement par le talon de la lame ou beaucoup plus rarement par l'aménagement d'une petite troncature transverse, rectiligne ou légèrement concave. Dans ce cas, la typologie des pièces se rapproche de celle des pointes de Malaurie, par exemple dans le locus 1 , avec 2 monopointes à base retouchée (fig. $48, n^{\text {os }} 4$ et 5). Elles restent exceptionnelles au sein de l'ensemble des armatures. On peut aussi noter la présence sur 6 pointes d'une coche basale opposée au bord abattu et bien différente d'une base, par exemple dans le locus 11 (fig. 35, $\mathrm{n}^{\circ} 2$ ). Cet aménagement confère aux armatures une forme rétrécie, qui aurait selon certains auteurs valeur chrono-culturelle: c'est la «penknife point» (Garrod, 1926), la pointe de Grundy ou encore le type 07 de G. Célérier (Célérier et al., 1993). Le tranchant des pointes porte rarement des retouches d'utilisation, même si dans 4 cas, on note une coche latérale ou encore une retouche abrupte qui dégage la pointe. L'utilisation de ces armatures comme pointe de flèche est attestée par de très nombreux types de fractures violentes: en premier lieu des fractures orthogonales apicales, une «base» à esquillements inverses, des fractures longitudinales en pseudo-coup de burin ou encore une fracture esquillée. Il y a enfin 12 cassures intervenues lors du façonnage du dos; ce sont des fractures de type pseudomicroburin Krukowski, identifiables sur les armatures et plus rarement sur les lames ou éclats à bord abattu.

Parmi l'ensemble des pièces à dos, nous n'avons déterminé qu'une seule lamelle à dos au sens strict (dans la zone 12), alors que ces outils existent souvent en faible pourcentage dans les assemblages aziliens classiques. C'est encore une fois la fragmentation importante du matériel qui nous a conduit à une grande prudence avec cette catégorie, très 
proche de celle des pièces à bord abattu. Nous ne voulions pas créer un groupe artificiel dont les caractères typologiques ne sont pas assez distincts. La quasi-absence de dos rectiligne et la largeur importante des pièces à bord abattu nous ont confortés dans cette attitude. De plus, nous n'avons pas mis en évidence une recherche de supports lamellaires dans les intentions du débitage. Au Magdalénien ou dans les groupes post-aziliens, où les lamelles à dos constituent une catégorie typologique importante, une chaîne opératoire lamellaire est mise en œuvre à la suite ou indépendamment du débitage de lames. Ce n'est pas le cas sur les sites aziliens et on peut légitimement s'interroger sur la validité de cet ensemble typologique pour ces cultures dans l'Ouest.

Toutes ces pointes forment donc un ensemble homogène qui permet de caler chronologiquement l'ensemble des locus dans une phase récente de l'Azilien. La présence de quelques pièces à base retouchée pourrait tirer la datation vers une phase légèrement plus récente, ainsi dans le locus 1. Inversement, les rares bipointes de la zone 5 (fig. 24) ou de la zone 8 (locus 11) nous entraîneraient vers des horizons plus anciens. On voit bien toutes les limites de cette approche typologique stricte sur des corpus segmentés en unités spatiales homogènes.

\section{L'outillage commun}

\section{Les grattoirs}

Les grattoirs sont parmi les outils du fonds commun les plus standardisés; la charge culturelle est à l'évidence forte dans la définition de ces outils (fig. 25, 36, 37 et 49). Les grattoirs sont réalisés principalement sur éclats courts, avec un rapport longueur/largeur peu dispersé (fig. 26). Les longueurs de ces outils sont faibles (entre $20 \mathrm{~mm}$ et $35 \mathrm{~mm}$ ), pour des largeurs comprises entre $18 \mathrm{~mm}$ et $28 \mathrm{~mm}$. Ces dimensions correspondent bien à des supports courts et larges, sans oublier toutefois que ces deux variables ont $\mathrm{pu}$ être corrigées par la retouche et par la cassure par flexion du support, fréquemment observée et probablement liée à un emmanchement. En cela, l'exigence concernant la longueur des supports n'était pas forcément très importante lors du débitage. Comprise entre $5 \mathrm{~mm}$ et $9 \mathrm{~mm}$, l'épaisseur des grattoirs est le paramètre le plus révélateur d'une standardisation, puisqu'elle n'a pas pu être modifiée par la retouche (fig. 27). Ce critère est semble-t-il important dans la définition du support adéquat, notamment si on le confronte à la grande latitude observée parmi les burins; il est fort probable, alors, que les grattoirs soient emmanchés.
Une chaîne opératoire d'éclats, parfois postérieure au débitage de lames, parfois indépendante, parfois intégrée, a été mise en œuvre afin d'obtenir des supports de grattoirs calibrés.

Le front du grattoir est circulaire ou unguiforme. La retouche limitée à l'extrémité distale s'étend exceptionnellement sur les bords du support. Le front, nettement convexe est aménagé par des enlèvements courts, exceptionnellement à tendance lamellaire. Ils sont présents partout, sauf dans le locus 3 (mais ce dernier ne contient que 170 pièces).

\section{Les burins}

Les burins sont généralement un peu moins nombreux que les grattoirs, sauf dans les locus 3, 13 et 40. Il s'agit essentiellement de burins sur cassure ou sur pan naturel (4\% de l'outillage commun), puis de burins sur troncature $(2,7 \%)$ (fig. 38 et 50). Dans ce cas, les troncatures préalables au coup de burin sont rectilignes ou légèrement concaves. La partie active de l'outil est le plus souvent latérale. Les burins dièdres d'axe sont très rares $(1 \%)$. On dénombre enfin quelques burins multiples, ainsi que des burins opposés à des grattoirs.

La comparaison entre les supports des grattoirs et ceux des burins permet de voir la standardisation des premiers par rapport à la dispersion des seconds (fig. 28 et 29), réalisés sur tous les types de support, éclat ou lame, de plein débitage ou non. En conséquence, une grande diversité des dimensions peut être observée: la longueur des burins varie entre $30 \mathrm{~mm}$ et $60 \mathrm{~mm}$ et leur largeur entre $10 \mathrm{~mm}$ et $35 \mathrm{~mm}$. De même, il y a une absence totale de corrélation entre la longueur et la largeur des pièces.

\section{Les éclats et les lames à bord abattu}

Nous avons rassemblé dans ces types toutes les pièces présentant un dos mais ne pouvant pas être qualifiées d'armatures, soit à cause de leurs dimensions (largeur supérieure à $15 \mathrm{~mm}$ ), soit en raison de leur morphologie (fig. 40). Elles forment une catégorie assez importante avec $5,7 \%$ de l'outillage commun, réparties entre lames et éclats. On peut distinguer plusieurs types de bord abattu: courbe, rectiligne ou irrégulier. Les supports de ces pièces, étudiés en comparaison avec ceux des pointes aziliennes, montrent qu'une partie d'entre eux pourrait être à rapprocher des armatures: comme le montrent d'ailleurs des analyses fonctionnelles (De Bie, Caspar, 2000), la césure n'est pas toujours stricte entre outils de boucherie et armatures. 

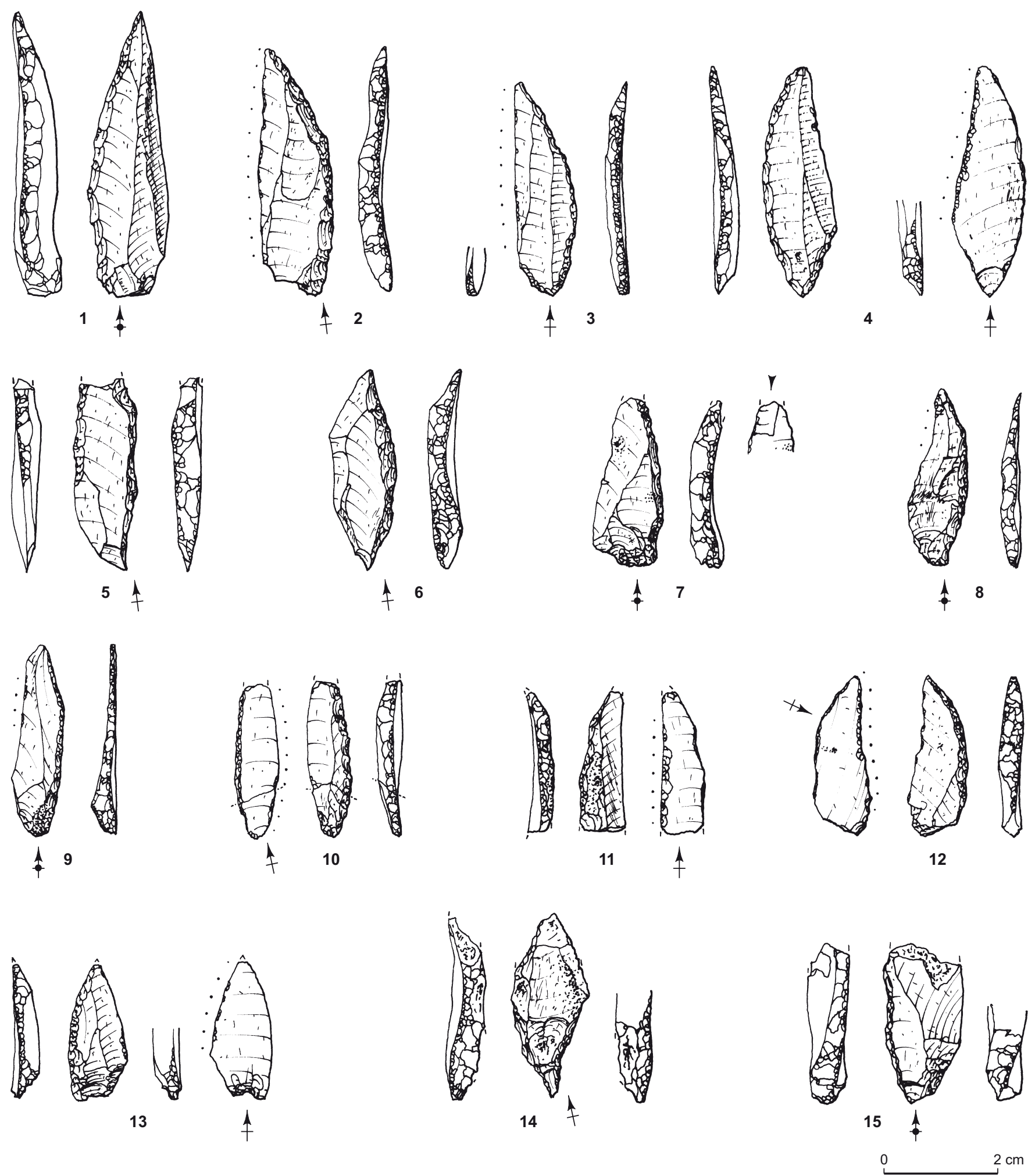

Fig. 24 - Mozé-sur-Louet «Les Chaloignes», zone 5 (locus azilien). 1-3, 7-10, 12 et 13, monopointes aziliennes; 4 et 6, bipointes aziliennes; 5 et 11, pointes aziliennes; 14 et 15, pièces à bord abattu (dessins: P. Forré, Inrap). 

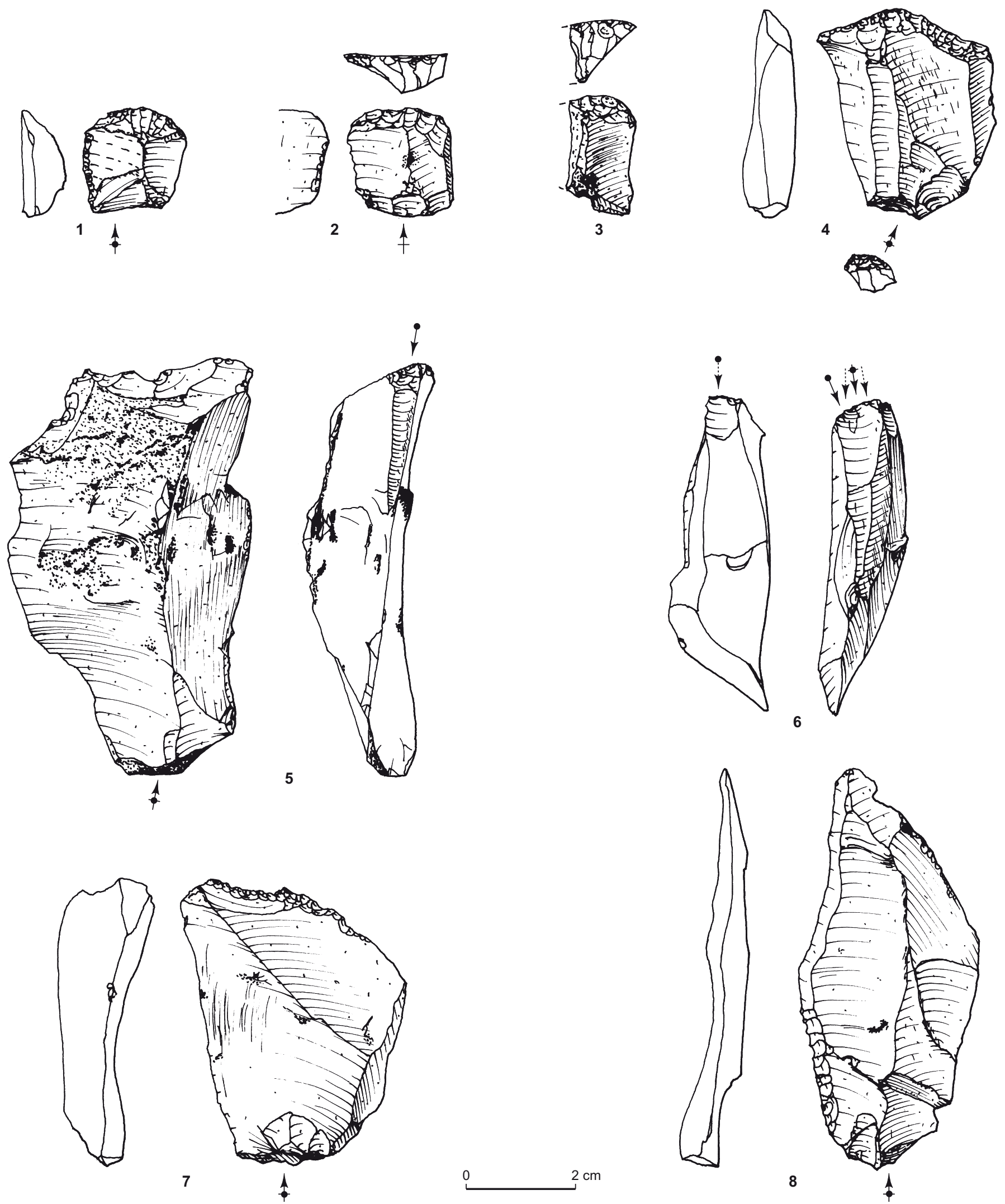

Fig. 25 - Mozé-sur-Louet «Les Chaloignes», locus 4. 1-4, grattoirs; 5, burin sur troncature; 6, burin sur cassure; 7, éclat aménagé; 8, lame aménagée (dessins: P. Forré, Inrap). 


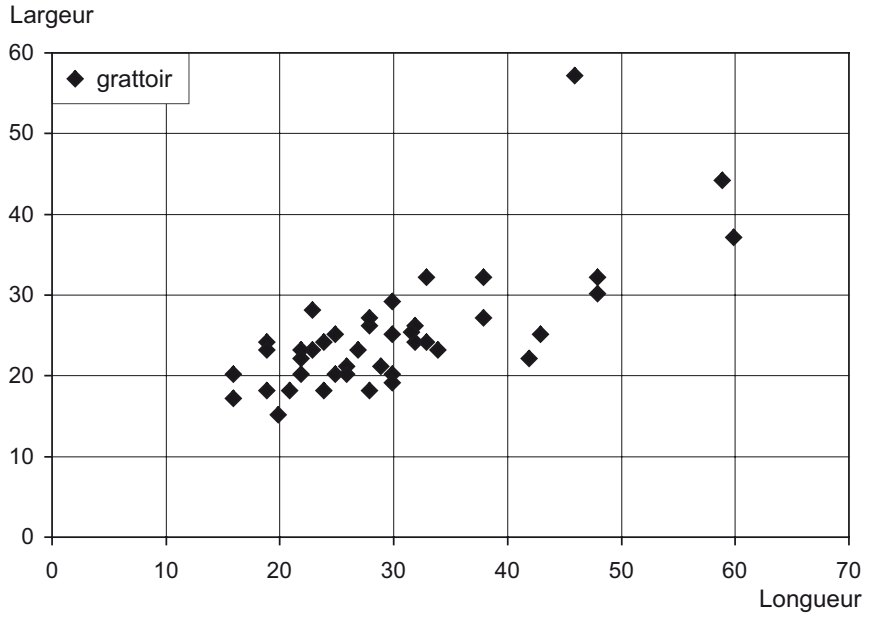

Fig. 26 - Longueur/largeur des grattoirs sur éclat (en mm).

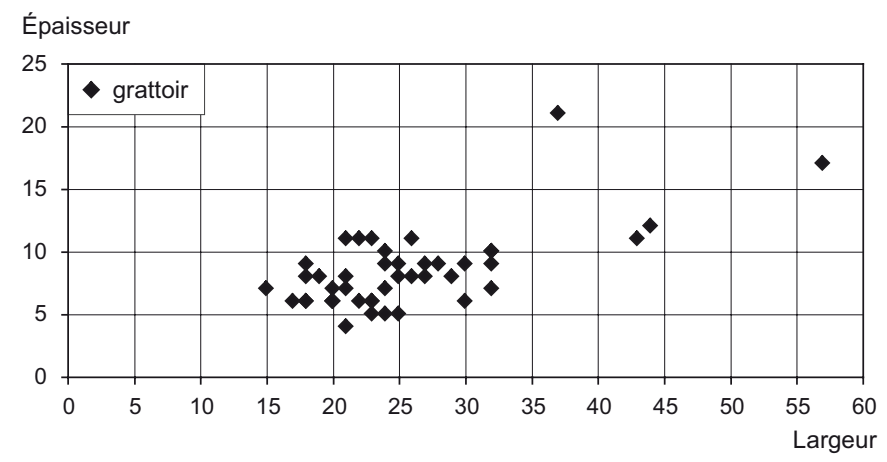

Fig. 27 - Largeur/épaisseur des grattoirs sur éclat (en mm).

\section{Les pièces tronquées}

La classe des troncatures est particulièrement paradoxale: il s'agit parfois des outils aménagés les plus nombreux (ainsi dans les locus 2, 4, 10, et 13), mais la diversité de leurs caractères techniques nous incite souvent à les négliger. Les supports sont des éclats ou des lames, plus rarement des lamelles. Ils sont issus de toutes les phases des chaînes opératoires, hors le plein débitage (fig. 51). Il s'agit donc de produits hétérogènes, irréguliers, souvent partiellement corticaux, de faibles dimensions. Les troncatures sont plutôt transverses (pour les deux tiers) qu'obliques, et plutôt rectilignes que concaves.

\section{Les lames et lamelles aménagées ou ébréchées}

Si le taux d'utilisation des lames de plein débitage est le plus important de tous les supports (fig. 30, 43 et 51), c'est en bonne part à cause des lames à fil ébréché ou aménagées (13,2\% de l'outillage) et dans une moindre mesure des lamelles $(5,2 \%)$. Par ailleurs, au sein de l'en-

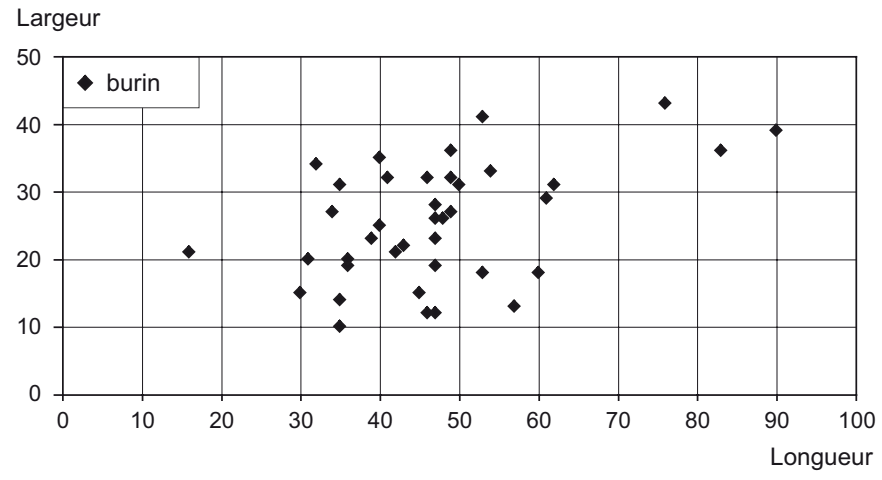

Fig. 28 - Longueur/largeur des burins (en mm).

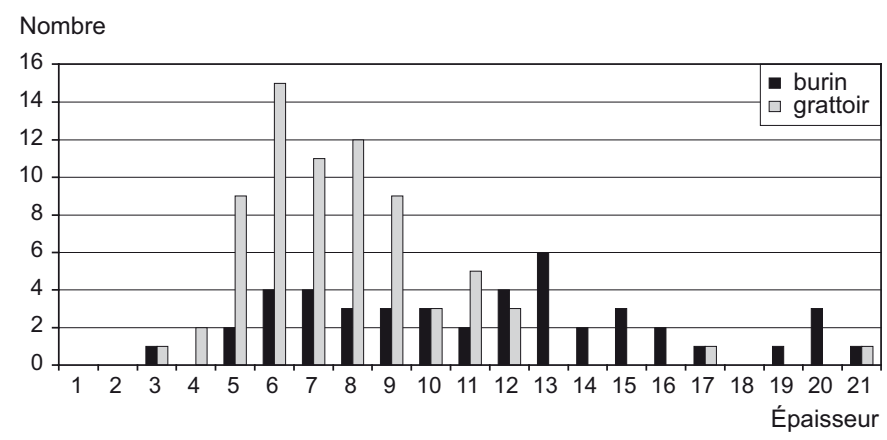

Fig. 29 - Histogramme des épaisseurs de burins et de grattoirs.

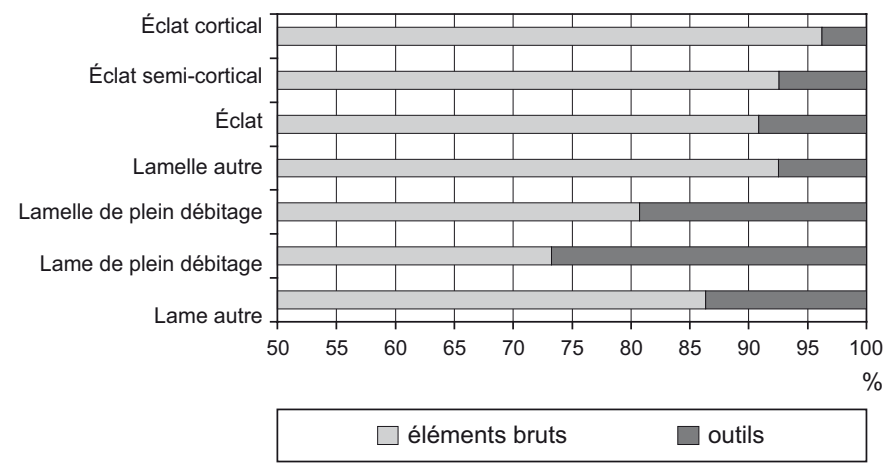

Fig. 30 - Taux d'utilisation des principaux supports, dans les locus aziliens homogènes (locus 1, 2, 3, 4, 11, 12, 13, 40).

semble laminaire, ce sont les supports les plus larges et les moins réguliers qui ont été aménagés, ce qui montre une fois de plus l'importance de la retouche pour la calibration des outils dans ce système technique. Lorsque la lame brute correspondait aux besoins des aziliens, elle fut utilisée telle quelle; dans le cas d'un support plus irrégulier, une légère retouche est venue modifier la partie active de l'outil. Notons que les supports bruts sans traces visibles semblent rarement utilisés sur le locus 1, selon l'étude de S. Philibert (cf. infra). 


\section{Les éclats aménagés ou ébréchés}

Nous avons établi une distinction entre les éclats aménagés et les éclats ébréchés: les premiers témoignent d'un aménagement évident du support par une retouche régulière sur un ou deux bords (fig. 51). Les seconds, marqués par un léger esquillement du bord, peuvent indiquer une utilisation de l'éclat comme outil mais peuvent aussi être le résultat de processus taphonomiques indépendants de l'action humaine. Ils sont donc moins à même de nous apporter des informations sur les intentions du débitage. L'existence d'une chaîne opératoire d'éclats confirme la présence d'un outillage raisonné sur éclat. L'étude des éclats aménagés doit nous permettre de savoir si les éclats les plus calibrés n'ont servi qu'à la fabrication des grattoirs ou s'ils ont servi plus largement pour tout l'outillage aménagé sur éclat. Nous avons déjà remarqué le faible éventail des épaisseurs pour les grattoirs; leur superposition avec les éclats aménagés et ébréchés révèle la faible standardisation de ces derniers par rapport aux grattoirs. En revanche, il n'y a pas d'épaisseur minimum pour les éclats retouchés. Les largeurs des éclats sont dispersées, comprises entre $2 \mathrm{~mm}$ et $11 \mathrm{~mm}$. La similitude entre les histogrammes des largeurs des éclats aménagés et des éclats ébréchés témoigne de l'inexistence d'une sélection différenciée. Pour les éclats aménagés, la retouche n'avait pas pour finalité de calibrer particulièrement les largeurs, puisque l'on ne note aucune valeur remarquable, mais plutôt de favoriser la prise en main ou l'emmanchement de l'outil.

Il faut noter ici l'absence de pièce esquillée dans les locus aziliens. Deux de ces outils sont répertoriés sur le site, dans des structures gauloises: dans l'ensemble de fosses du vallon occidental (fait 18, US 34.16) et dans une base de paroi de la maison gauloise (fait 69, US 85.7). Ce ne sont pas des ensembles clos et il est impossible de les attribuer à l'une des composantes culturelles qui jonchent la zone 4 .

\section{Les percuteurs}

Les percuteurs, au nombre de 12, sont présents uniquement dans trois zones, dans la moitié sud du site (zones 5, 8 et 11), avec un important regroupement dans le locus 10 . Ces outils sont pour la plupart des galets d'un grès-quartzite très dense et au grain très fin. Les stigmates de la taille aux deux extrémités sont peu développés: il s'agit à l'évidence de galets d'accès facile qui peuvent être renouvelés. Cornéenne, quartz, silex de type G, ont également été mis à contribution, avec un unique exemplaire comme témoignage. Les percuteurs ont pour la plupart une forme allongée, avec des longueurs comprises entre $56 \mathrm{~mm}$ et $128 \mathrm{~mm}$ et une moyenne de $93 \mathrm{~mm}$.

\section{ORIENTATION DE LA PRODUCTION}

\section{Les objectifs du débitage}

Le processus de taille donne naissance à des supports divers et, à ce stade de l'étude, la comparaison de leur taux d'utilisation permet d'éviter de les classer suivant une hiérarchie inadéquate, toute emprunte des critères esthétiques du préhistorien. Et dans ce système de valeurs, les lames de plein débitage sont de loin les supports les plus convoités, avec un taux d'utilisation de 26,7\%, suivi par les lamelles avec 19,3\% (tabl. VII et VIII). La présence d'un fil ébréché sur les éclats croit en raison inverse de la proportion de cortex sur leur face supérieure: $3,8 \%$ pour les éclats corticaux, 7,4\% pour les semi-corticaux et $9,2 \%$ pour les éclats sans cortex (fig. 30). Ces valeurs beaucoup plus fortes pour les supports allongés donnent une première orientation de la production. Lors de l'étude des outils, nous avons vu ce qui sous-tendait cette demande. Les deux types d'outils les plus calibrés, et donc les plus investis techniquement, sont les armatures et les grattoirs. Ils correspondent à deux sortes de supports bien différenciés, d'une part des lames étroites et rectilignes, d'autre part des éclats courts et assez minces.

Les lames de plein débitage sont courtes et assez épaisses (fig. 43). Elles sont le plus souvent à deux pans avec des bords à tendance parallèle, plutôt rectilignes sans jamais être très réguliers. La rectitude du profil est une caractéristique recherchée, comme le montrent les lames et les tables laminaires des nucléus. Les talons sont généralement minces et lisses, quel que soit le type de support (tabl. IX). On constate tout de même un soin plus particulier apporté aux lames de plein débitage avec un facettage dans près de $19 \%$ des cas, avec de grandes variations entre locus (de 6,1\% à 68,8\%). C'est un caractère qui doit être souligné car il reflète des choix techniques aziliens. La comparaison avec d'autres sites aziliens devrait nous permettre de dire s'il s'agit d'une caractéristique largement partagée à l'échelle de la France, ou s'il peut s'agir d'un trait plus régional. Au début de notre étude, nous avons établi une distinction entre lame et lamelle, autour d'une valeur classique de $12 \mathrm{~mm}$, afin de percevoir éventuellement des différences dans la gestion et la morphologie de ces deux types de support. Mais en réalité, il n'y a pas de projet lamellaire autonome. Les 
Tabl. VII - Quelques taux des principales concentrations de silex.

\begin{tabular}{|c|c|c|c|c|c|c|c|c|c|c|c|c|c|c|}
\hline & Taux & 1 & 2 & 3 & 4 & 10 & 11 & 12 & 13 & 40 & Zone 3 & Zone 5 & Zone 12 & Zone 13 \\
\hline État & \% de brûlé & 45,6 & 46,4 & 24,8 & 20,2 & 34,2 & 52,6 & 24,9 & 24,6 & 41,2 & 25,6 & 40,3 & 60,3 & 21,7 \\
\hline Matière & $\begin{array}{l}\text { \% de silex roulé parmi les } \\
\text { identifiés }\end{array}$ & 81,8 & 95,5 & 98,4 & 39,1 & 75,1 & 90,3 & 83,3 & 98,6 & 94,7 & 99,3 & 95,1 & 94,3 & 97,6 \\
\hline \multirow{2}{*}{ Armature } & armatures / outils aménagés & 59,1 & 53,8 & 40,0 & 21,7 & 23,4 & 29,3 & 15,4 & 33,3 & 23,8 & 31,3 & 24,8 & 31,4 & 22,2 \\
\hline & armatures / outils & 32,9 & 25,0 & 20,0 & 12,5 & 11,6 & 16,2 & 5,3 & 18,2 & 12,8 & 11,6 & 11,5 & 25,4 & 9,1 \\
\hline \multirow{2}{*}{ Grattoirs } & grattoirs / outils aménagés & 18,2 & 15,4 & 0,0 & 21,7 & 16,9 & 20,0 & 25,0 & 8,3 & 14,3 & 12,5 & 20,8 & 14,3 & 44,9 \\
\hline & grattoirs / outils & 10,1 & 7,1 & 0,0 & 12,5 & 8,4 & 11,0 & 7,9 & 4,5 & 7,7 & 4,7 & 9,6 & 7,9 & 18,2 \\
\hline \multirow{2}{*}{ Burins } & burins / outils aménagés & 15,9 & 0,0 & 40,0 & 8,7 & 9,1 & 8,0 & 15,4 & 25,0 & 42,9 & 6,3 & 4,7 & 20,0 & 0,0 \\
\hline & burins / outils & 8,9 & 0,0 & 20,0 & 5,0 & 4,5 & 4,4 & 5,3 & 13,6 & 23,1 & 2,2 & 2,3 & 11,1 & 0,0 \\
\hline \multirow{2}{*}{ Outils } & $\begin{array}{l}\text { outils communs / totalité du } \\
\text { locus }\end{array}$ & 6,5 & 5,8 & 9,5 & 12,5 & 12,4 & 9,4 & 10,2 & 7,3 & 9,9 & 13,1 & 9,2 & 8,5 & 6,8 \\
\hline & $\begin{array}{l}\text { outils communs + armatures / } \\
\text { totalité du locus }\end{array}$ & 9,6 & 7,7 & 11,9 & 14,2 & 14,0 & 11,2 & 10,7 & 8,9 & 11,3 & 14,8 & 10,3 & 11,3 & 7,5 \\
\hline \multirow{2}{*}{ Lame } & $\begin{array}{l}\text { taux large (tous fragments + } \\
\text { outils / totalité du locus) }\end{array}$ & 16,3 & 11,5 & 15,5 & 24,5 & 12,5 & 11,4 & 12,4 & 26,6 & 9,8 & 14,8 & 11,1 & 7,3 & - \\
\hline & $\begin{array}{l}\text { taux restreint (tous fragments } \\
\text { - outils /totalité du locus) }\end{array}$ & 13,8 & 10,2 & 13,5 & 23,1 & 10,9 & 9,3 & 11,0 & 24,3 & 7,2 & 11,7 & 9,6 & 6,0 & 10,7 \\
\hline \multirow{2}{*}{ Lamelle } & $\begin{array}{l}\text { taux large (tous fragments + } \\
\text { outils / totalité du locus) }\end{array}$ & 13,3 & 9,3 & 8,9 & 16,4 & 6,5 & 10,0 & 7,6 & 10,5 & 7,8 & 10,9 & 8,1 & 10,2 & - \\
\hline & $\begin{array}{l}\text { taux restreint (tous fragments } \\
\text { - outils / totalité du locus) }\end{array}$ & 12,5 & 8,4 & 8,8 & 16,1 & 6,0 & 8,8 & 7,9 & 11,0 & 7,2 & 10,5 & 7,2 & 9,5 & 6,1 \\
\hline \multirow{2}{*}{ Éclat simple } & $\begin{array}{l}\text { taux large (tous fragments + } \\
\text { outils / totalité du locus) }\end{array}$ & 44,1 & 44,5 & 43,5 & 37,7 & 49,7 & 52,4 & 47,5 & 33,5 & 43,5 & 42,3 & 42,4 & 43,7 & - \\
\hline & $\begin{array}{l}\text { taux restreint (tous fragments } \\
\text { - outils / totalité du locus) }\end{array}$ & 45,7 & 44,9 & 43,9 & 38,0 & 49,2 & 53,5 & 45,9 & 33,6 & 43,5 & 43,5 & 42,5 & 44,7 & 38,6 \\
\hline \multirow{2}{*}{ Éclat cortical } & $\begin{array}{l}\text { taux large (tous fragments + } \\
\text { outils / totalité du locus) }\end{array}$ & 11,7 & 10,2 & 7,1 & 3,2 & 9,6 & 7,0 & 9,0 & 7,3 & 8,7 & 10,7 & 8,7 & 10,0 & - \\
\hline & $\begin{array}{l}\text { taux restreint (tous fragments } \\
\text { - outils / totalité du locus) }\end{array}$ & 12,4 & 10,4 & 7,4 & 3,7 & 10,6 & 7,5 & 9,8 & 8,0 & 9,5 & 8,1 & 9,1 & 9,7 & 11,1 \\
\hline \multirow{2}{*}{ Talon facetté } & $\begin{array}{l}\text { facettage sur lame de plein } \\
\text { débitage }\end{array}$ & 31,1 & 68,8 & 22,2 & 38,5 & 6,1 & 26,4 & 15,8 & 37,0 & 31,3 & 13,3 & 5,8 & 33,3 & - \\
\hline & facettage sur éclat simple & 9,4 & 17,8 & 8,7 & 14,3 & 5,1 & 10,4 & 5,8 & 28,8 & 37,5 & 3,4 & 4,5 & 5,8 & - \\
\hline \multirow{2}{*}{ Abrasion } & $\begin{array}{l}\text { abrasion sur lame de plein } \\
\text { débitage }\end{array}$ & 65,6 & 43,8 & 88,9 & 30,8 & 44,1 & 32,1 & 57,9 & 37,0 & 46,2 & 53,3 & 37,0 & 53,3 & - \\
\hline & abrasion sur éclat simple & 42,9 & 33,3 & 56,5 & 28,6 & 33,5 & 26,5 & 28,8 & 28,8 & 37,0 & 31,0 & 19,3 & 23,0 & - \\
\hline
\end{tabular}

nucléus laminaires sont souvent abandonnés alors que les derniers enlèvements se situent autour de $40 \mathrm{~mm}$ de long et $10 \mathrm{~mm}$ de large. Dans le locus 1 ou le locus 11, où l'échantillonnage a été particulièrement contrôlé, les largeurs des produits bruts allongés s'étalent entre $7 \mathrm{~mm}$ et $17 \mathrm{~mm}$. Le prélèvement pour les armatures concerne des calibres supérieurs à $12 \mathrm{~mm}$. Que deviennent les pièces plus petites, si abondantes? On ne les observe pas dans le reste de l'outillage aménagé et ce sont les produits les plus larges qui dominent nettement parmi les lames et lamelles ébréchées (moyenne des largeurs: 18,4 mm). Dernier élément de diagnose: sous une valeur de $9 \mathrm{~mm}$ de large, la proportion de lamelles irrégulières croit fortement, aux dépens des produits réguliers, témoignage du faible intérêt pour ces modules qui s'insèrent probablement dans un débitage de lames plus larges.
Ces observations convergent vers une image de production peu standardisée de produits allongés. La grande variété morphométrique des supports d'armatures et de grattoirs indique une grande souplesse dans les exigences aziliennes; la retouche joue un rôle important dans l'aspect terminal de l'outil. Pour le reste de l'outillage, on notera la place importante qu'occupent les troncatures et les burins par rapport aux grattoirs dans certains locus. Ils semblent être attachés à des produits de seconde intention que l'on tentera de définir pour chaque locus : cet apparent désordre peut en effet dissimuler des intentions plus éphémères mais néanmoins standardisées. Pour les burins, la longueur et surtout l'épaisseur du produit comptent avant tout. Les éclats sans cortex dominent de peu en nombre de supports les éclats semi-corticaux et les lames de plein débitage; on rencontre même deux tablettes, un casson et un nucléus. 
Tabl. VIII - Les produits de débitage bruts par locus ou par zone, en nombre (colonne de gauche) et en proportions (colonne de droite).

\begin{tabular}{|c|c|c|c|c|c|c|c|c|c|c|c|c|c|c|c|c|c|c|c|c|c|c|c|c|c|c|c|c|}
\hline \multirow{3}{*}{$\begin{array}{c}\text { N / Locus } \\
\text { Éclat cortical }\end{array}$} & \multirow{2}{*}{\multicolumn{2}{|c|}{1}} & \multirow{2}{*}{\multicolumn{2}{|c|}{2}} & \multirow{2}{*}{\multicolumn{2}{|c|}{3}} & \multirow{2}{*}{\multicolumn{2}{|c|}{4}} & \multirow{2}{*}{\multicolumn{2}{|c|}{10}} & \multirow{2}{*}{\multicolumn{2}{|c|}{11}} & \multirow{2}{*}{\multicolumn{2}{|c|}{12}} & \multirow{2}{*}{\multicolumn{2}{|c|}{13}} & \multirow{2}{*}{\multicolumn{2}{|c|}{40}} & \multirow{2}{*}{\multicolumn{2}{|c|}{ Zone 3}} & \multirow{2}{*}{\multicolumn{2}{|c|}{ Zone 5}} & \multirow{2}{*}{\multicolumn{2}{|c|}{ Zone 12}} & \multirow{2}{*}{\multicolumn{2}{|c|}{ Zone 13}} & \multicolumn{2}{|c|}{ Total } \\
\hline & & & & & & & & & & & & & & & & & & & & & & & & & & & \multirow{2}{*}{\begin{tabular}{|l|}
$N$ \\
680
\end{tabular}} & \multirow{2}{*}{\begin{tabular}{|c|}
$\%$ \\
9,2
\end{tabular}} \\
\hline & 92 & 12,4 & 35 & 10,4 & 11 & 7,4 & 8 & 3,3 & 101 & 10,6 & 81 & 7,5 & 31 & 9,8 & 18 & 8,0 & 29 & 9,5 & 20 & 8,1 & 254 & 9,1 & 51 & 10,2 & 33 & 11,1 & & \\
\hline Éclat semi-cortical & 46 & 6,2 & 21 & 6,3 & 12 & 8,1 & 25 & 10,4 & 99 & 10,4 & 90 & 8,4 & 21 & 6,6 & 16 & 7,1 & 29 & 9,5 & 25 & 10,1 & 432 & 15,4 & 44 & 8,8 & 41 & 13,8 & 816 & 11,0 \\
\hline Éclat & 340 & 46,0 & 152 & 45,2 & 65 & 43,9 & 91 & 37,8 & 471 & 49,3 & 576 & 53,7 & 146 & 46,2 & 78 & 34,5 & 133 & 43,5 & 108 & 43,5 & 1191 & 42,5 & 229 & 45,7 & 115 & 38,6 & 3351 & 45,3 \\
\hline $\begin{array}{l}\text { Lame de plein } \\
\text { débitage }\end{array}$ & 74 & 10,0 & 19 & 5,7 & 15 & 10,1 & 34 & 14,1 & 69 & 7,2 & 74 & 6,9 & 21 & 6,6 & 34 & 15,0 & 16 & 5,2 & 16 & 6,5 & 152 & 5,4 & 23 & 4,6 & 11 & 3,7 & 524 & 7,1 \\
\hline Lame autre & 28 & 3,8 & 15 & 4,5 & 5 & 3,4 & 24 & 10,0 & 35 & 3,7 & 26 & 2,4 & 14 & 4,4 & 21 & 9,3 & 6 & 2,0 & 13 & 5,2 & 118 & 4,2 & 7 & 1,4 & 21 & 7,0 & 305 & 4,1 \\
\hline $\begin{array}{l}\text { Lamelle de plein } \\
\text { débitage }\end{array}$ & 67 & 9,1 & 20 & 6,0 & 11 & 7,4 & 31 & 12,9 & 35 & 3,7 & 65 & 6,1 & 16 & 5,1 & 15 & 6,6 & 15 & 4,9 & 19 & 7,7 & 149 & 5,3 & 19 & 3,8 & 8 & 2,7 & 443 & 6,0 \\
\hline Lamelle autre & 25 & 3,4 & 8 & 2,4 & 2 & 1,4 & 9 & 3,7 & 22 & 2,3 & 29 & 2,7 & 9 & 2,8 & 10 & 4,4 & 7 & 2,3 & 7 & 2,8 & 63 & 2,3 & 27 & 5,4 & 10 & 3,4 & 191 & 2,6 \\
\hline Chute de burin & 2 & 0,3 & 0 & 0,0 & 1 & 0,7 & 2 & 0,8 & 2 & 0,2 & 2 & 0,2 & 0 & 0,0 & 1 & 0,4 & 5 & 1,6 & 1 & 0,4 & 9 & 0,3 & 1 & 0,2 & 1 & 0,3 & 25 & 0,3 \\
\hline Crête & 0 & 0,0 & 0 & 0,0 & 0 & 0,0 & 0 & 0,0 & 1 & 0,1 & 0 & 0,0 & 1 & 0,3 & 0 & 0,0 & 0 & 0,0 & 0 & 0,0 & 0 & 0,0 & 0 & 0,0 & 0 & 0,0 & 2 & 0,0 \\
\hline Néo-crête & 10 & 1,4 & 5 & 1,5 & 3 & 2,0 & 1 & 0,4 & 4 & 0,4 & 5 & 0,5 & 1 & 0,3 & 2 & 0,9 & 4 & 1,3 & 3 & 1,2 & 6 & 0,2 & 1 & 0,2 & 1 & 0,3 & 44 & 0,6 \\
\hline Tablette & 7 & 0,9 & 2 & 0,6 & 3 & 2,0 & 2 & 0,8 & 8 & 0,8 & 10 & 0,9 & 1 & 0,3 & 1 & 0,4 & 2 & 0,7 & 4 & 1,6 & 24 & 0,9 & 7 & 1,4 & 4 & 1,3 & 64 & 0,9 \\
\hline Nucléus à lame & 5 & 0,7 & 7 & 2,1 & 3 & 2,0 & 1 & 0,4 & 8 & 0,8 & 8 & 0,7 & 5 & 1,6 & 10 & 4,4 & 4 & 1,3 & 2 & 0,8 & 30 & 1,1 & 3 & 0,6 & 2 & 0,7 & 83 & 1,1 \\
\hline Nucléus à éclat & 5 & 0,7 & 10 & 3,0 & 3 & 2,0 & 0 & 0,0 & 15 & 1,6 & 26 & 2,4 & 13 & 4,1 & 2 & 0,9 & 5 & 1,6 & 2 & 0,8 & 63 & 2,3 & 9 & 1,8 & 7 & 2,3 & 144 & 1,9 \\
\hline $\begin{array}{l}\text { Nucléus } \\
\text { Indéterminé }\end{array}$ & 2 & 0,3 & 1 & 0,3 & 0 & 0,0 & 0 & 0,0 & 1 & 0,1 & 5 & 0,5 & 1 & 0,3 & 1 & 0,4 & 3 & 1,0 & 0 & 0,0 & 16 & 0,6 & 2 & 0,4 & 0 & 0,0 & 30 & 0,4 \\
\hline Casson & 36 & 4,9 & 41 & 12,2 & 14 & 9,5 & 13 & 5,4 & 85 & 8,9 & 76 & 7,1 & 36 & 11,4 & 17 & 7,5 & 48 & 15,7 & 27 & 10,9 & 293 & 10,5 & 78 & 15,6 & 44 & 14,8 & 686 & 9,3 \\
\hline Bloc & 0 & 0,0 & 0 & 0,0 & 0 & 0,0 & 0 & 0,0 & 0 & 0,0 & 0 & 0,0 & 0 & 0,0 & 0 & 0,0 & 0 & 0,0 & 1 & 0,4 & 0 & 0,0 & 0 & 0,0 & 0 & 0,0 & 1 & 0,0 \\
\hline Total & 739 & 100 & 336 & 100 & 148 & 100 & 241 & 100 & 956 & 100 & 1073 & 100 & 316 & 100 & 226 & 100 & 306 & 100 & 248 & 100 & 2800 & 100 & 501 & 100 & 298 & 100 & 7389 & 100 \\
\hline
\end{tabular}

Tabl. IX - Proportions de talons des principaux produits de débitage, issus des locus homogènes $(1,2,3,4,11,12,13$ et $40-1929$ individus $)$.

\begin{tabular}{|l|c|c|c|c|c|c|c|c|}
\hline \multicolumn{1}{|c|}{ Débitage } & Cortical & Dièdre & Écrasé & Facetté & Filiforme & Large lisse & Mince lisse & Punctiforme \\
\hline Éclat & 6,2 & 5,5 & 11,1 & 9,9 & 6,4 & 17,6 & 27,9 & 15,3 \\
\hline Éclat cortical & 18,8 & 1,8 & 17,6 & 6,1 & 1,2 & 23,0 & 18,2 & 13,3 \\
\hline Éclat semi-cortical & 9,9 & 6,3 & 9,4 & 9,4 & 3,7 & 30,9 & 19,4 & 11,0 \\
\hline Lame autre & 9,7 & 5,8 & 4,9 & 13,6 & 4,9 & 24,3 & 24,3 & 12,6 \\
\hline Lame de plein débitage & 4,9 & 4,5 & 2,2 & 29,5 & 3,1 & 17,0 & 30,8 & 8,0 \\
\hline Lamelle autre & 1,6 & 6,5 & 12,9 & 9,7 & 8,1 & 9,7 & 29,0 & 22,6 \\
\hline Lamelle de plein débitage & 6,1 & 2,7 & 8,1 & 15,5 & 10,1 & 3,4 & 35,8 & 18,2 \\
\hline Néo-crête & 0,0 & 0,0 & 5,6 & 22,2 & 0,0 & 33,3 & 27,8 & 11,1 \\
\hline Tablette & 0,0 & 0,0 & 9,5 & 19,0 & 0,0 & 23,8 & 33,3 & 14,3 \\
\hline Total (en \%) & $\mathbf{7 , 4}$ & $\mathbf{4 , 9}$ & $\mathbf{9 , 9}$ & $\mathbf{1 2 , 6}$ & $\mathbf{5 , 4}$ & $\mathbf{1 8 , 5}$ & $\mathbf{2 7 , 1}$ & $\mathbf{1 4 , 2}$ \\
\hline
\end{tabular}

Pour la confection de cet outil, il y a donc un prélèvement de supports au gré du débitage, avec peut-être des intentions très ponctuelles difficiles à matérialiser sans remontage spécifique. En tout état de cause, il n'est pas possible de parler de séquence de débitage particulière pour ces outils.

\section{Aspects quantitatifs}

Dans la production peu standardisée des Chaloignes, il est d'ailleurs souvent difficile d'établir une distinction entre les phases de mise en forme et la phase de plein débitage. Nous nous sommes donc contentés de séparer les lames de plein débitage et le reste de la production laminaire. Pour celles-ci, nous avons tenu compte de la régularité des bords de la lame, de ses dimensions et de la présence ou non de cortex. Ces critères correspondent aux observations effectuées sur les supports d'armatures qui illustrent le mieux les exigences techniques aziliennes. Les lames de plein débitage représentent $10 \%$ de la totalité du débitage auxquelles il faut ajouter $4 \%$ de sous-produits laminaires. Nous 
avons décompté $7 \%$ de lamelles de plein débitage et près de $3 \%$ de lamelles autres. Au total, les produits lamino-lamellaires forment $23,8 \%$ de l'ensemble du matériel. Ce taux augmente à $27,2 \%$ si l'on considère le nombre minimum d'individus (NMI calculé uniquement à partir des pièces entières et des fragments proximaux, bruts et retouchés), cette légère augmentation traduisant peut-être une sousévaluation de ces supports dès lors qu'ils sont fractionnés ou trop retouchés.

Les éclats représentent près de $55 \%$ du débitage et il y a $8,7 \%$ d'éclats corticaux (le NMI pour les locus homogènes est respectivement de $53 \%$ et 9,3\%). Si, dans l'ensemble, la productivité des nucléus à éclats est faible, on constate une standardisation importante des éclats. La maîtrise de la chaîne opératoire d'éclats est manifeste et il y a une vraie phase de plein débitage destinée à l'obtention de supports de grattoirs bien calibrés. Mais en définitive, ces dimensions reflètent aussi les limites des petits galets de Loire.

En l'absence de remontages importants, l'estimation de la productivité réelle des blocs est difficile. Si l'on rapporte le nombre de pièces brutes (exprimées en NMI) aux nucléus, il y aurait 4,1 lames de plein débitage par nucléus à lames (14,6 éclats de plein débitage par nucléus à éclats) ; cette approximation est, dans les faits, fort discutable, car nous ne connaissons qu'un état terminal des nucléus. Les séries laminaires remontées montrent un débitage maximum d'une dizaine de lames, quelques fois de seulement 5 ou 6 produits. Les débitages semblent donc dans l'ensemble peu poussés, si l'on en juge par les dimensions résiduelles importantes de nombreux blocs. En fin de production la longueur moyenne d'un nucléus à lame est de $47,9 \mathrm{~mm}$, très proche de celle des nucléus à éclats (43,3 mm). La rentabilité de la matière est plutôt faible, ce qui pourrait illustrer une adéquation assez stricte entre les besoins et la production, sans anticipation à moyen ou long terme. Le débitage brut serait alors essentiellement le rejet d'une production qui a servi les intentions du tailleur, production peu rentable mais suffisante pour répondre à des besoins précis et pensés sur le court terme. Cet abandon rapide n'est pas lié uniquement à des problèmes techniques, mais bien à une abondance des galets et surtout à l'exigence de grandes dimensions laminaires. Il faut quand même souligner une gestion de la matière à plus long terme que nous avons mis en évidence sur quelques locus. Il y a par exemple une absence presque totale de nucléus laminaire sur certains locus où le débitage de lames avait été particulièrement important. Il semble alors que les nucléus bien calibrés ont été emportés hors de la zone, en vue d'une utili- sation ultérieure. Dans ces cas là, il y aurait un vrai souci de conserver des volumes pouvant encore servir les intentions du débitage.

\section{DYNAMIQUE DU DÉBITAGE}

Plusieurs problèmes se posent à l'issue de l'étude des produits de débitage; leur résolution devrait permettre de décrire plus en profondeur le techno-complexe azilien. D'une part, les liens éventuels entre la séquence laminaire et la séquence à éclat restent à comprendre. Il convient d'autre part d'expliquer la faible standardisation des produits laminaires, en interrogeant l'architecture des volumes débités. Il est enfin nécessaire de saisir comment le tailleur a adapté les normes du système technique azilien à la conformation initiale des blocs, en amont de la chaîne opératoire. L'étude de la gestion des volumes est issue d'une part des remontages, d'autre part des schémas diacritiques réalisés sur les nucléus. Ces derniers ne sont hélas pas très caractéristiques du débitage laminaire et une part d'ombre existe sur les rythmes d'exploitation pour l'obtention des grandes lames.

\section{Organisation du volume}

L'ouverture des galets de Loire réguliers est assurée au moyen d'entames corticales qui s'adaptent généralement aux nervures naturelles des volumes (fig. 31). Les plans de frappe ainsi préparés ont un angle de chasse ouvert, souvent proche de $90^{\circ}$. De nombreux galets de silex gélifs ont explosé dès les premiers coups de percuteurs, comme en témoignent particulièrement des pièces des locus 10 , 11 ou 12. Les fragments ont ensuite été utilisés suivant les mêmes principes que les galets plus réguliers, à savoir une recherche de la plus grande longueur. Facile à remonter, ces fragments révèlent des dimensions de galets dépassant parfois les $130 \mathrm{~mm}$, bien qu'en règle générale, ils mesurent autour de $80 \mathrm{~mm}$. Cette petite taille nécessite une économie de matière dans la phase de mise en forme du bloc, afin d'obtenir les supports les plus allongés possibles. La table laminaire est généralement implantée dans le sens longitudinal du bloc. Selon les locus, on constate que le choix de la face large ou étroite des galets n'est pas systématique. Les flancs et le dos ne portent jamais de traces de crêtes latérales ou dorsales qui pourraient indiquer une vraie mise en forme des blocs avant ou pendant le débitage. De la même façon, les crêtes d'entame sont très rares, une simple arête corticale suffit à l'initiation du débitage, voire l'angle 

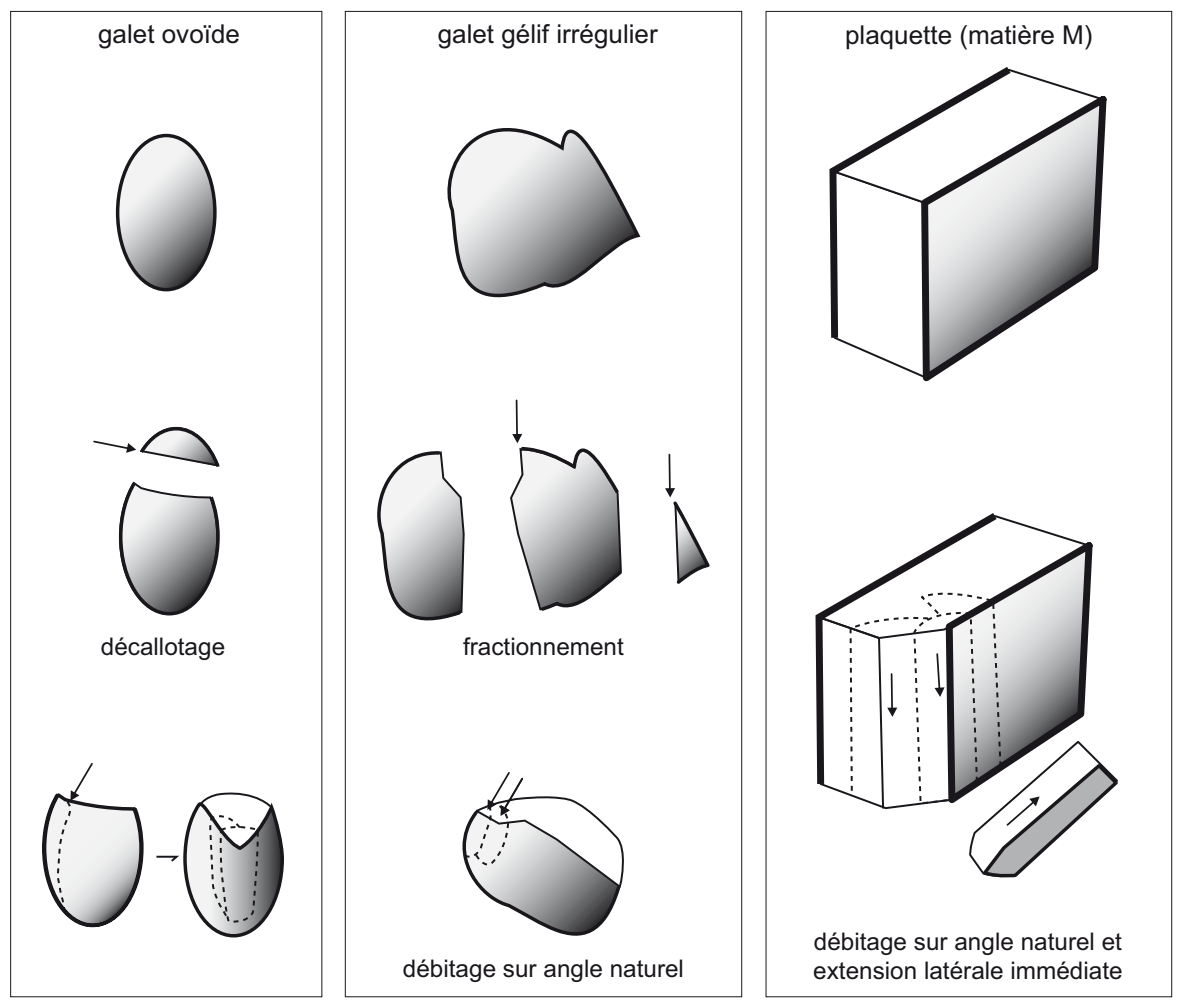

Fig. 31 - Adaptation des séquences d'entame suivant la morphologie du bloc, dans les débitages aziliens des Chaloignes (dessin: G. Marchand, CNRS UMR 6566).

favorable d'un casson. Le débitage est conduit à l'aide d'un percuteur de pierre dure. Étant donné l'angle du plan de frappe, la percussion est rentrante et non tangentielle. On constate donc une proportion très importante de talons larges sur les supports. Autre cas de figure, les plaquettes de matière $\mathrm{M}$ (silcrète) ont connu un débitage suivant leur litage, à partir d'un plan de frappe orthogonal aux niveaux crayeux: le premier enlèvement est dans ce cas une lame semi-corticale, suivie par d'autres de même nature (fig. 32). La table laminaire ainsi construite s'étend donc largement sur une face, avec un recul oblique qui profite au début de deux tables.

\section{La séquence laminaire au sein d'une chaîne opératoire intégrée}

Si l'implantation de la table profite souvent d'une surface rétrécie, le débitage s'étend rapidement sur un flanc (fig. 44 et 45). Comme en témoignent parfaitement certains nucléus du locus 1 , le recul peut se faire de manière oblique, alternativement sur une surface puis sur l'autre. Il y a alors deux tables qui forment un angle favorable pour le débitage. Il est fréquent alors de constater sur les nucléus que la table la plus large (l'ex-flanc) donne des lames larges ou des éclats, alors que la table rétrécie fournit des lames plus étroites. La faible calibration des supports que l'on observe n'est probablement pas sans rapport avec ce rythme d'exploitation. Il n'y a aucun aménagement des flancs hormis des enlèvements d'axe et cette partie du volume n'a pas de fonctionnement autonome. Par ailleurs, le débitage n'est jamais périphérique et le dos est très rarement exploité. L'essentiel du traitement du volume revient donc à la table et à son fonctionnement.

Le débitage est majoritairement bipolaire; environ $65 \%$ des nucléus portent deux plans de frappe opposés. Il existe, d'une part un vrai débitage bipolaire avec deux plans de frappe ayant fonctionné alternativement (fig. 44, $\mathrm{n}^{\mathrm{o}} 1$ ), d'autre part un débitage où les plans de frappe sont hiérarchisés (fig. 44, $\mathrm{n}^{\circ} 4$ ). Lorsqu'il s'agit d'un vrai débitage bipolaire, on constate que les plans de frappe sont parallèles, et très rarement orthogonaux ou sécants ce qui permet de ménager la plus grande longueur pour les produits. Ils ont servi successivement à exploiter une même table, l'alternance des plans de frappe intervenant après chaque courte série laminaire unipolaire. La périodicité de ce basculement est difficile à estimer, mais en phase terminale, chaque séquence comprendrait moins de 8 enlèvements. Cette méthode a l'avantage de permettre un entretien 

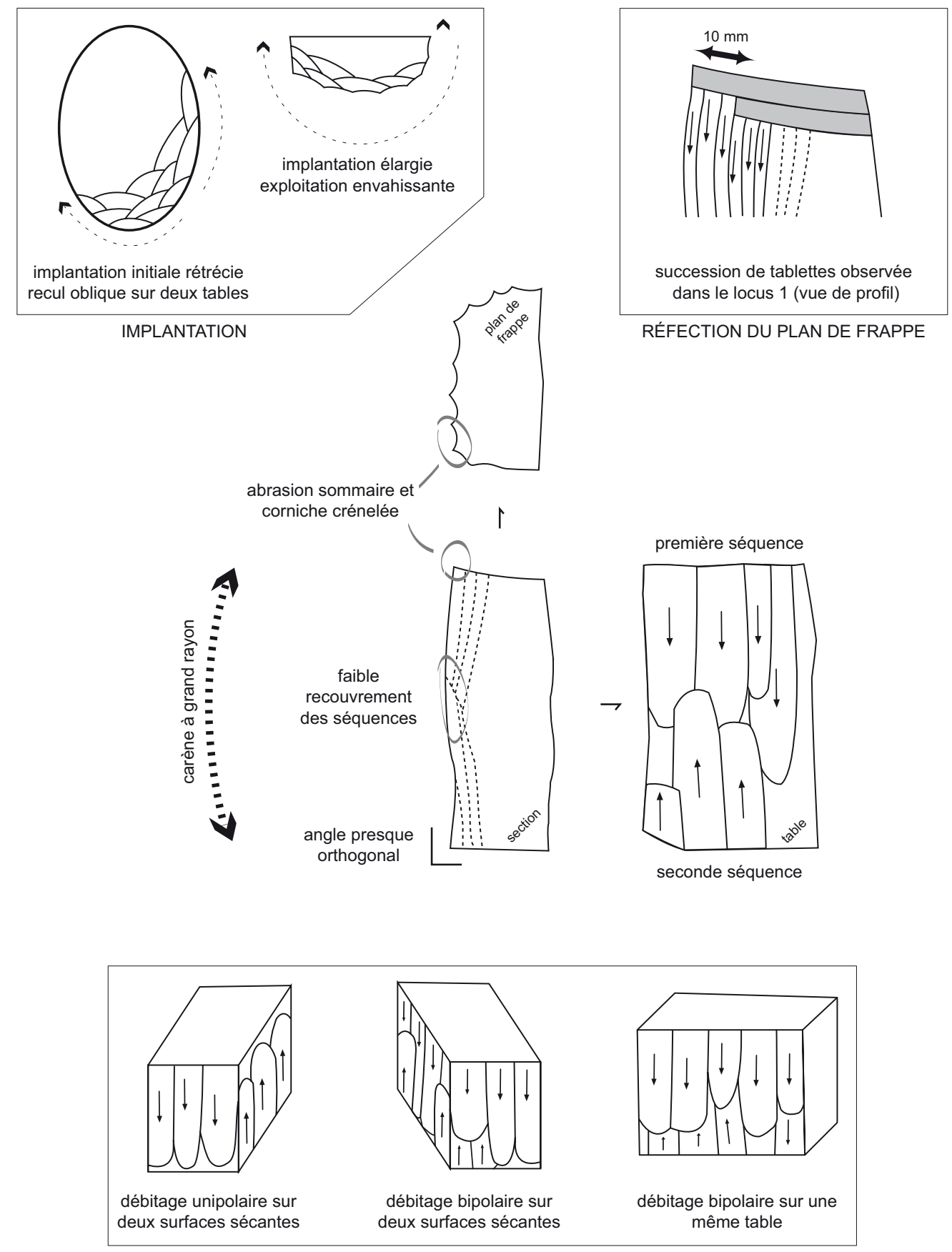

MODALITÉS DU DÉBITAGE BIPOLAIRE

Fig. 32 - Représentation schématique de la gestion des volumes dans le débitage azilien des Chaloignes (dessin: G. Marchand, CNRS UMR 6566).

automatique de la table et de sa carène, même s'il y a un faible recouvrement en extrémité distale des produits. La table du nucléus reste assez plate, permettant l'obtention de produits rectilignes, tout en nettoyant les éventuels accidents par des enlèvements d'axe. Cette absence de convexité longitudinale conduit à de nombreux réfléchissements au cours du débitage, phénomène accentué par l'utilisation de percuteur de pierre dure. Ces accidents liés au débitage sont le plus souvent effacés à partir du plan de frappe opposé. À cet effet, certains nucléus présentent un 
plan de frappe secondaire, dont la fonction semble être uniquement l'intervention technique et non la pleine exploitation laminaire. Une modalité de débitage bipolaire peut être décrite sur le locus 1, qui se marie avec le recul oblique sur deux tables. Si, dans ce cas de figure, chaque plan de frappe commande de manière préférentielle une des tables sécantes (et donc non identiques), il n'est pas possible de parler de deux séquences unipolaires autonomes, car les interactions sont fréquentes entre elles et l'on retrouve une architecture du nucléus semblable aux autres. Enfin, une implantation sur une face large et allongée est très souvent observée avec une exploitation qui va sans cesse du centre au bord de la table, les flancs n'existant guère. Il peut s'agir pour les petits nucléus de l'aboutissement logique d'un débitage oblique sur deux tables, mais on l'observe aussi sur les nucléus de grands modules du locus 13. La production d'éclats intervient nécessairement dans ce système, notamment sur la surface la plus large; en cela il convient de parler de chaîne opératoire intégrée.

Le niveau technique du débitage est moyen et les gestes techniques visant à entretenir les angles du nucléus sont rares. Les crêtes destinées à l'entretien sont anecdotiques et le plus souvent partielles. Le cintre du nucléus n'est pas un paramètre fondamental, comme l'indique l'implantation de la table sur la face large du bloc. Les éclats de flanc sont anecdotiques, puisqu'il y a peu d'interventions techniques à partir des flancs ou du dos du nucléus. En fin d'exploitation, la table devient très plate et les flancs ont disparu. La réorientation du nucléus au cours du débitage est très rare. Elle intervient parfois sur les volumes qui ont fait l'objet de l'exploitation la plus intensive, soit que le nucléus provienne d'une matière exogène, soit que la qualité du bloc soit particulièrement bonne.

L'entretien des plans de frappe n'est pas fréquent; des tablettes ont cependant été retrouvées, par exemple dans le locus 1 pour la production laminaire. Les talons sont le plus souvent lisses, car le ravivage des plans de frappe se fait à l'aide d'éclats épais qui emportent la totalité de la surface. L'abrasion est rare, généralement peu marquée et les surplombs occasionnés par les enlèvements antérieurs sont fréquemment conservés ce qui donne un aspect denticulé au bord de plan de frappe. Ce sont les lames et les lamelles de plein débitage qui ont fait l'objet de l'abrasion la plus fréquente (respectivement $44 \%$ et $32,4 \%$ ). Il faut souligner que la mention de ce procédé n'indique pas toujours un soin particulier apporté au bord de plan de frappe. Il est en effet fréquent que cette abrasion soit très légère et ne borde même pas toute la largeur du talon. L'utilisation préféren- tielle du percuteur de pierre dure est partiellement responsable de l'irrégularité, comme en témoignent les esquilles de dimensions très diverses et souvent rebroussées.

\section{Les séquences à éclats}

Plusieurs nucléus portent exclusivement un débitage d'éclats, sans première phase laminaire. Le schéma opératoire mis en œuvre est le même que celui utilisé pour les lames, avec très souvent une exploitation bipolaire sur des tables assez larges envahissants les flancs (fig. 44, no 3 ). La régularité de certains supports de grattoirs implique une continuité de ces séquences. Les nucléus ont une très faible productivité. Beaucoup ont été abandonnés sur les locus, alors que les dimensions résiduelles du bloc permettaient de continuer le débitage et qu'aucun accident n'était venu défigurer la table. Il y a donc un comportement plus opportuniste dans la gestion des nucléus à éclats, que dans celle des nucléus laminaires. Si les moyens techniques mis en œuvre pour l'obtention des éclats sont en conformité avec le reste de la production, la gestion à très court terme de ces blocs montre une hiérarchie effective entre la production des lames et celle des éclats: l'emport de nucléus à éclats n'a jamais été mis en évidence aux Chaloignes. Ces principes ne s'opposent pas à l'hypothèse d'une chaîne opératoire intégrée. D'ailleurs, il est fréquent de rencontrer des nucléus laminaires repris en nucléus à éclats lors d'une dernière phase de débitage. Mais cette production d'éclats peut aussi intervenir - par hypothèse - en cours de débitage, avec le prélèvement d'éclats destiné à des outils au gré des besoins.

\section{CONGLUSIONS}

La recherche de supports particuliers, notamment pour les armatures, les grattoirs et dans une moindre mesure les burins, n'indique pas une stricte séparation des séquences de production. Le débitage de lames est peu productif, mais il permet l'obtention de supports présentant certaines caractéristiques morphologiques récurrentes: rectitude du profil, longueur réduite et largeur mal calibrée, qui sont autant de caractères contribuant à définir le système lithique des Chaloignes. L'étude de l'ensemble laminaire montre le faible rendement de chaque nucléus, mais aussi la bonne adéquation entre les besoins et la production. L'usage de deux plans de frappe, parfaitement adaptée à ces intentions, est un caractère culturel fort. Après la transformation des lames de plein débitage en armatures, il reste 
peu de supports bruts dans les locus, hormis les déchets. Il y a donc très peu d'anticipation des besoins, même si parfois les nucléus laminaires les plus rentables ne sont pas abandonnés à la fin du débitage, mais sont emportés par le tailleur pour un débitage ultérieur.

Le débitage d'éclats, orienté vers la production de supports courts et larges, est également peu productif. Une séquence standardisée est liée à la fabrication des grattoirs, le reste de l'outillage aménagé utilisant des supports plus variés. Les liens entre les deux sont cependant assez forts, puisqu'il n'y a pas de modalités exclusives à l'une ou l'autre des séquences. Le modèle technique que nous proposons comprend des séquences laminaires, souvent contrôlées par deux plans de frappe, et des séquences à éclats. Ces deux types de séquences peuvent être liées au sein d'une même chaîne, suivant la configuration des blocs, comme le révèlent les remontages et l'observation des nucléus. D'ailleurs, les taux d'abrasion sur les corniches des lames de plein débitage et des éclats non-corticaux sont corrélés (coefficient de corrélation de 0,865 pour l'ensemble des locus homogènes), ce qui pourrait nous signaler un processus linéaire. La corrélation est en revanche moins évidente pour le facettage (coefficient de 0,532). Dans tous les cas, le schème opératoire est le même. Il présente des caractéristiques techniques qui ne sont pas strictement liées à des contraintes matérielles, même si la conformation arrondie des blocs a facilité les premières opérations et si leurs petites dimensions réduisent automatiquement celles des supports. Il s'agit donc de choix aziliens, qui s'appliquent aussi bien aux lames qu'aux éclats, et qui révèlent la simplicité du débitage orienté vers une production peu rentable, répondant à des besoins à court terme.

G. Q. et S. S.

\section{ORGANISATION DES ACTIVITÉS DANS L'HABITAT}

\section{LE LOCUS 11: UNE AIRE D'ACTIVITÉS MULTIPLES}

Installé sur le schiste au milieu de la zone 8, le locus 11 est fondamental pour notre compréhension du site des Chaloignes, puisqu'il s'agit du locus homogène le plus abondant (fig. 33). Il a en outre livré 3 galets gravés qui permettent enfin de donner un contexte chrono-culturel clair à ces manifestations symboliques du val de Loire. Un

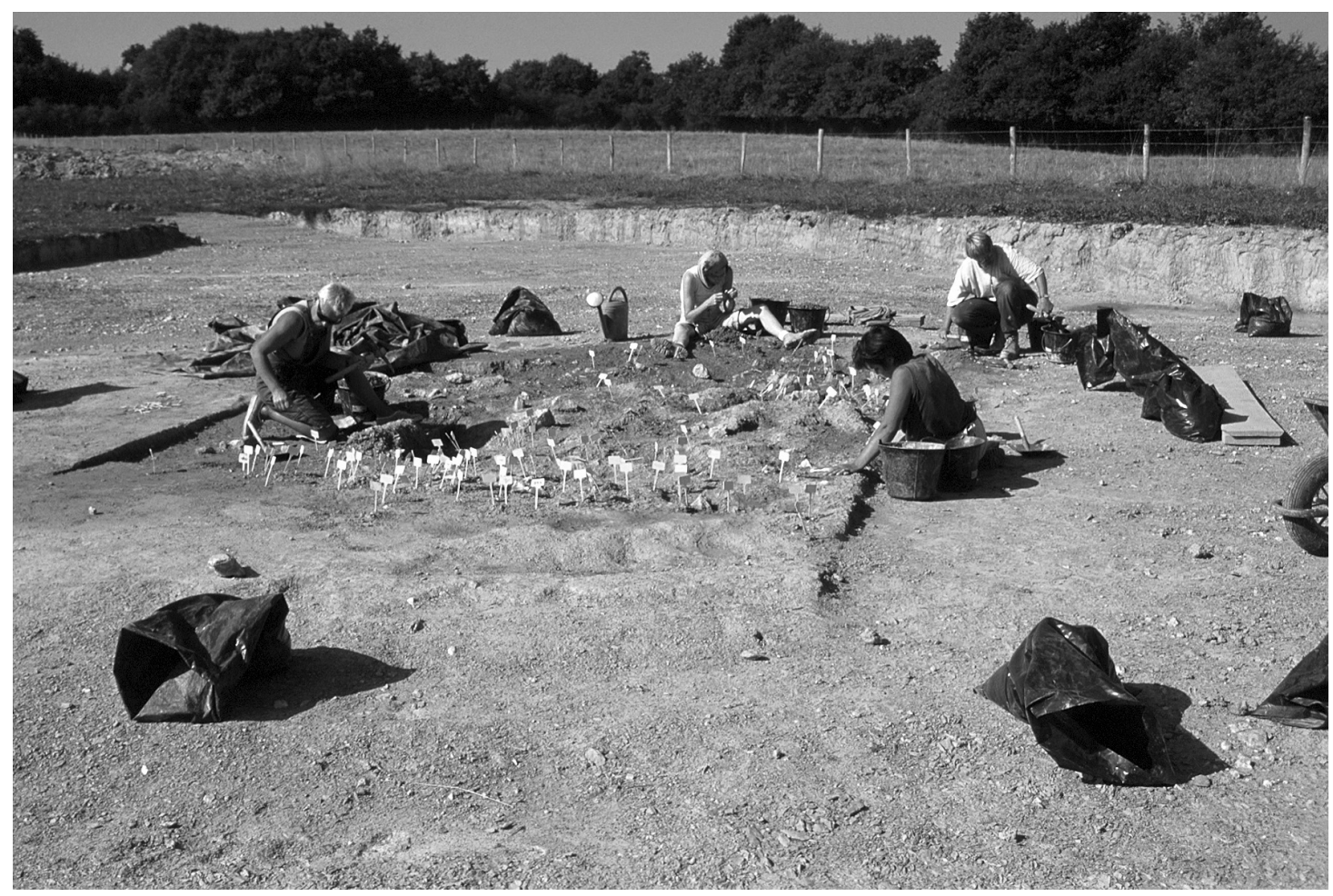

Fig. 33 - Le locus 11 en cours de fouille, vu du nord-est (cliché: G. Marchand, CNRS UMR 6566). 


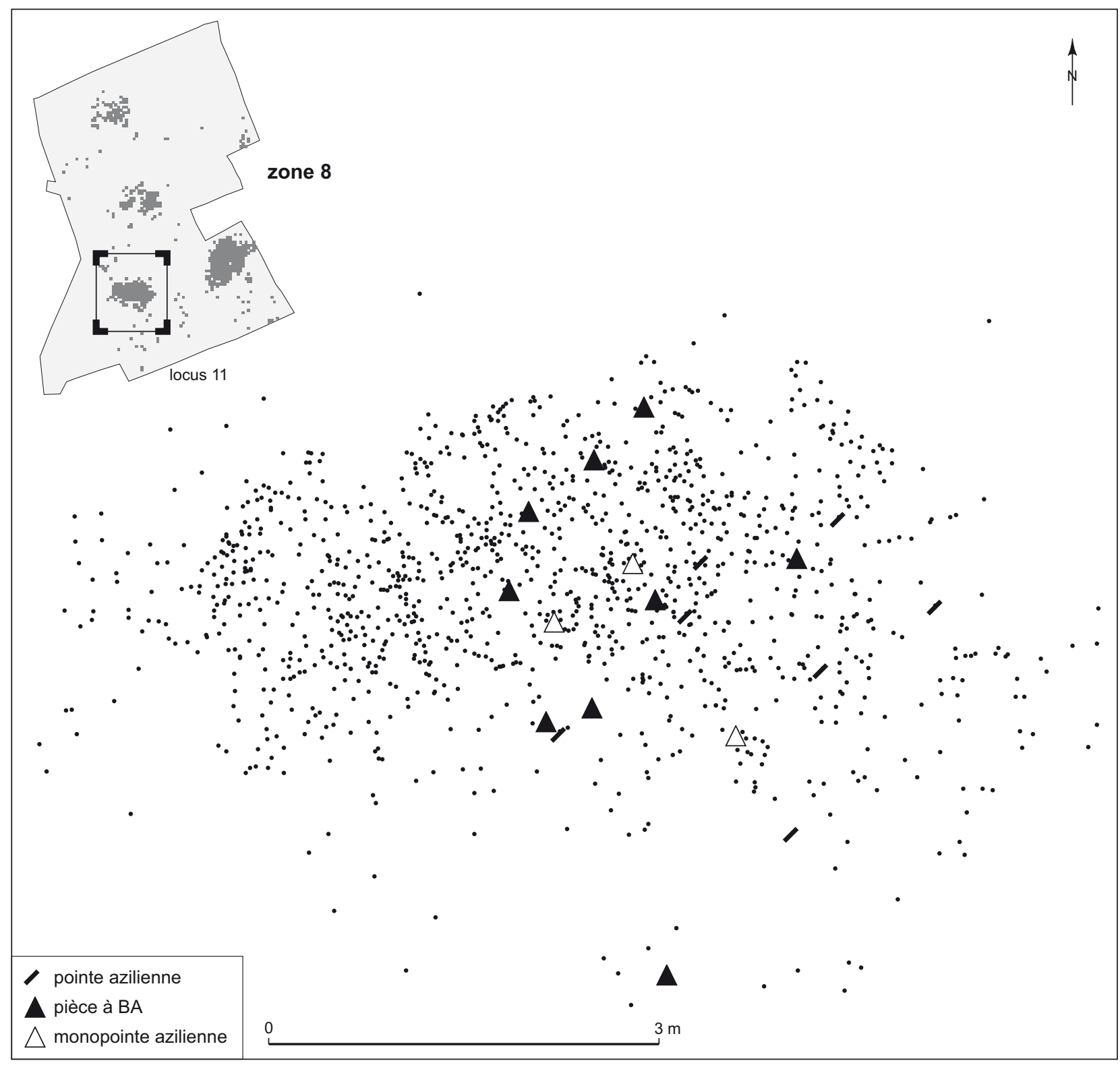

Fig. 34 - Locus 11, plan de répartition des armatures.

fragment de crayon d'ocre a également été découvert; son extrémité pointue, les facettes et les stries indiquent une utilisation intense pour colorer. L'étude typo-technologique de l'industrie lithique du locus 11 révèle des caractères statistiques très proches de la moyenne générale des locus aziliens, ce qui en fait l'amas le plus représentatif du site. Cette remarque n'a de sens que si on la relie aux activités réalisées sur le locus 11 et à leurs influences sur les variations quantitatives.

\section{RÉPARTITION SPATIALE}

Le locus 11 s'inscrit dans un polygone de forme assez allongée (6,60 m par 3,50 m), d'environ $35 \mathrm{~m}^{2}$ (fig. 34). L'essentiel des remontages a été réalisé sur une zone de $3 \mathrm{~m}^{2}$ au centre. Il a subi des coups de godets malencontreux sur ses limites septentrionales, lors du décapage mécanique. En règle générale, les pièces raccordées (blocs gélifs, débitage ou pièces cassées au feu) sont peu éloignées les unes des 
autres. Les raccords de pièces atteintes différemment par la chaleur sont très fréquents.

Le plan de densité des vestiges indique deux petites concentrations plus importantes de vestiges, à proximité l'une de l'autre. La répartition des matières premières présente quelques irrégularités, même si ces silex nonroulés restent minoritaires sur le locus. Ainsi, les silex en matière $\mathrm{G}$ sont concentrés dans la partie ouest du locus, où il y a un nucléus à lames et des éclats. Seules deux pièces en cette matière se trouvent à l'ouest du locus. Toutefois, le seul remontage effectué dans ce silex concerne un éclat sur un fragment de nucléus; cette absence signifie qu'il manque de nombreux produits intermédiaires. Il y a deux interprétations possibles: soit de nombreux produits de débitage ont été emportés hors du locus, soit le bloc a été taillé hors du locus. Seulement quelques pièces se retrouvent alors dans cette zone, à la suite d'un rejet ou au contraire pour une utilisation indéterminée. Par contraste, la matière $B$ est localisée plutôt à l'est. Les armatures occupent ellesaussi cette moitié orientale du locus, hors de la zone de concentration maximale des vestiges, et dans une moindre mesure les coches. Les remontages et raccords effectués entre ces deux zones nous permettent pourtant de conclure qu'il ne s'agit pas de la superposition de deux occupations successives, mais bien d'une même entité archéologique. D'ailleurs, les autres données analysées en termes spatiaux ne laissent pas voir d'organisation significative, qu'il s'agisse des burins, des grattoirs, des nucléus, des lames ou des produits corticaux.

\section{LA MATIÈRE PREMIÈRE}

Comme ailleurs, le silex a été récolté de manière préférentielle sur les bords de la Loire ou sur ses terrasses. Les pièces à cortex roulé représentent 39,2\% de l'ensemble, ce qui correspond à 90,3\% si l'on exclut les silex sans cortex. L'explosion de blocs gélifs pose quelques questions quant à l'identité des gens qui récoltaient les galets, puisque leur mauvaise qualité semblait assurée au son mat qu'il rendait, à moins que les tailleurs aient voulu récupérer de petits fragments aptes à la production. L'importation à partir des marges sédimentaires concerne donc environ $10 \%$ des pièces, parmi lesquelles le silex G se distingue. Dans cette partie sud du site, les locus 10 et 40, proches voisins du 11, en livraient également, mais pas le locus 12. Il faut signaler également la présence de quatre pièces en quartzite de Montbert, dont un burin sur cassure.

\section{LES OUTILS}

La proportion d'outils correspond à $11,2 \%$ des pièces cotées, ce qui est conforme à la moyenne générale. Les armatures sont toutes des pointes aziliennes, avec deux exemples possibles de bipointes (fig. 35, $\mathrm{n}^{\text {os }} 3$ et 4); les fragments de pièces à bord abattu sont assurément ici des fragments mésiaux ou proximaux de pointes. Sur ces fragments se devine dans deux cas une base retouchée convexe (fig. 35, $\mathrm{n}^{\text {os }} 16$ et 17 ). Le rétrécissement de la base par une retouche partielle est une modalité représentée (fig. $35, \mathrm{n}^{\text {os }} 2$ et 6 ). La latéralisation des pointes aziliennes à droite semble l'emporter ( 8 pour 3 ), mais cette absence d'exclusive nous semble révélatrice du peu d'intérêt de ce critère dans l'Azilien des Chaloignes. Les supports sont des lamelles larges et des lames étroites (entre $9 \mathrm{~mm}$ et $16 \mathrm{~mm}$ ) et assez épaisses (de $3 \mathrm{~mm}$ à $8 \mathrm{~mm}$ ), toutes issues du plein débitage et donc sans cortex. L'éventail assez large de ces dimensions est révélateur d'un calibrage modéré des produits. Il n'y a pas d'armatures microlithiques et ce malgré un tamisage à l'eau d'une part significative des sédiments.

Les grattoirs forment une autre catégorie assez standardisée dans cette industrie azilienne, par leurs dimensions et la nature de leur support (fig. 36 et 37). Ils sont réalisés pour la plupart sur éclats minces, courts et aux nervures régulières. Des cassures par flexion peuvent affecter le bord opposé au front (grattoirs raccourcis), ce qui laisse supposer des supports plus allongés, mais cela n'est pas la règle. Il n’y a pas de grattoirs circulaires; la forme en éventail ou unguiforme est majoritaire. On note aussi un grattoir double sur éclat allongé (fig. 37, $\mathrm{n}^{\circ} 2$ ) et deux grattoirs opposés à des burins sur pan naturel (fig. $36, \mathrm{n}^{\text {os }} 6$ et 10 ).

Les burins sur cassure ou sur pan naturel (fig. $38, n^{\text {os }} 3$ à 6) supplantent nettement en nombre les burins sur troncature (fig. $38, \mathrm{n}^{\text {os }} 1$ et 2 ). La fabrication des burins ne semble pas exiger beaucoup de régularité pour les supports, ils sont réalisés sur éclats courts ou plus allongés. Seul le burin sur troncature a bénéficié d'une lamelle de plein débitage comme support, le talon facetté de la lamelle ayant servi de plan de frappe pour le coup de burin (fig. 38, $\mathrm{n}^{\circ} 1$ ).

Le taux d'éclats à troncature transverse (fig. 37, no 4) est le plus important parmi les locus aziliens, mais l'industrie se rapproche de la norme générale si l'on prend en compte toutes les troncatures. Ces troncatures sont plutôt rectilignes $(n=6)$ que concaves $(n=2)$ ou denticulées $(n=2)$. La retouche est unipolaire et abrupte, sauf dans le cas d'un 

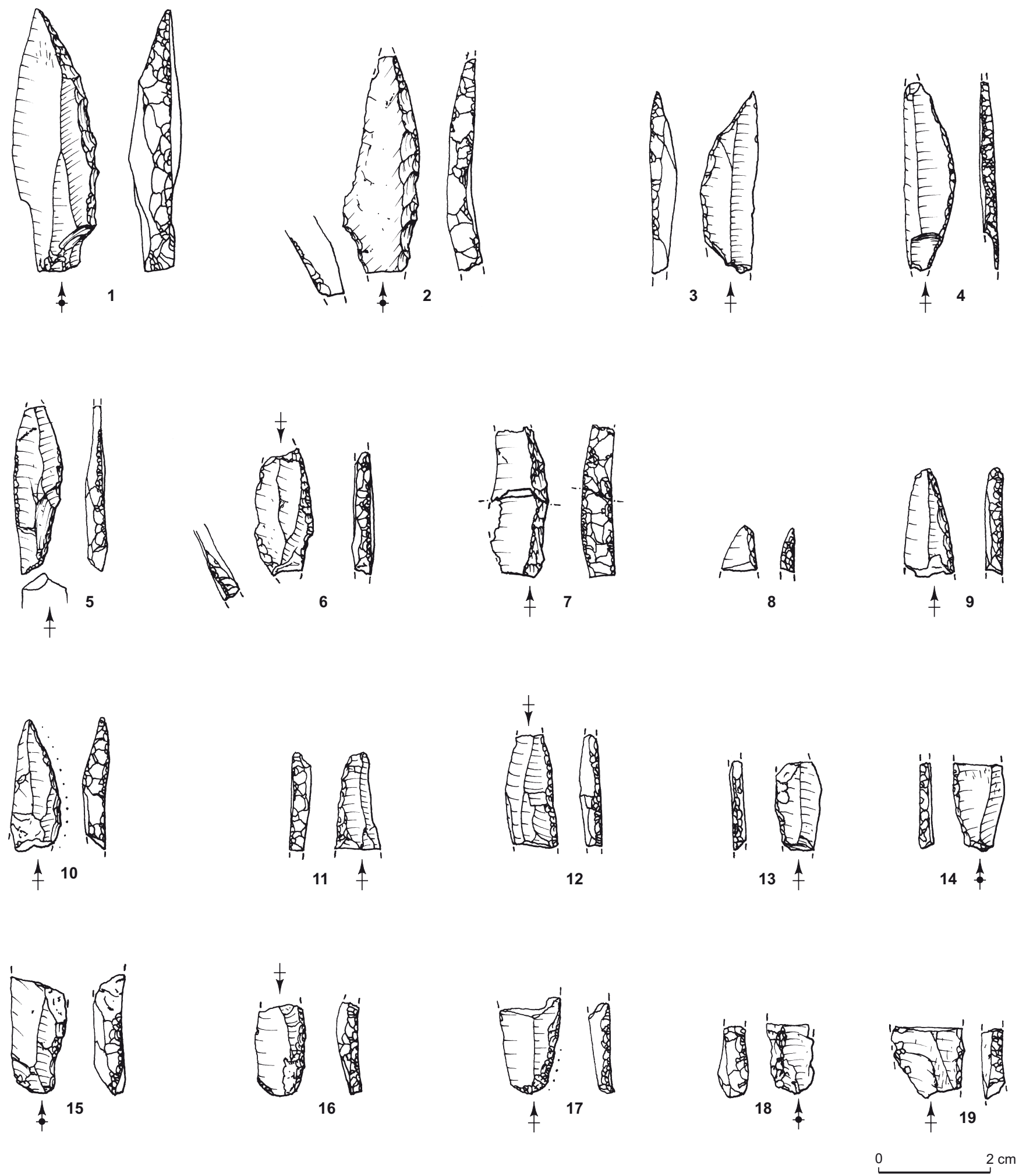

Fig. 35 - Les Chaloignes à Mozé-sur-Louet, locus 11. 1-10, pointes aziliennes entières ou fragmentées; 11-19, pièces à bord abattu (dessins: P. Forré, Inrap). 

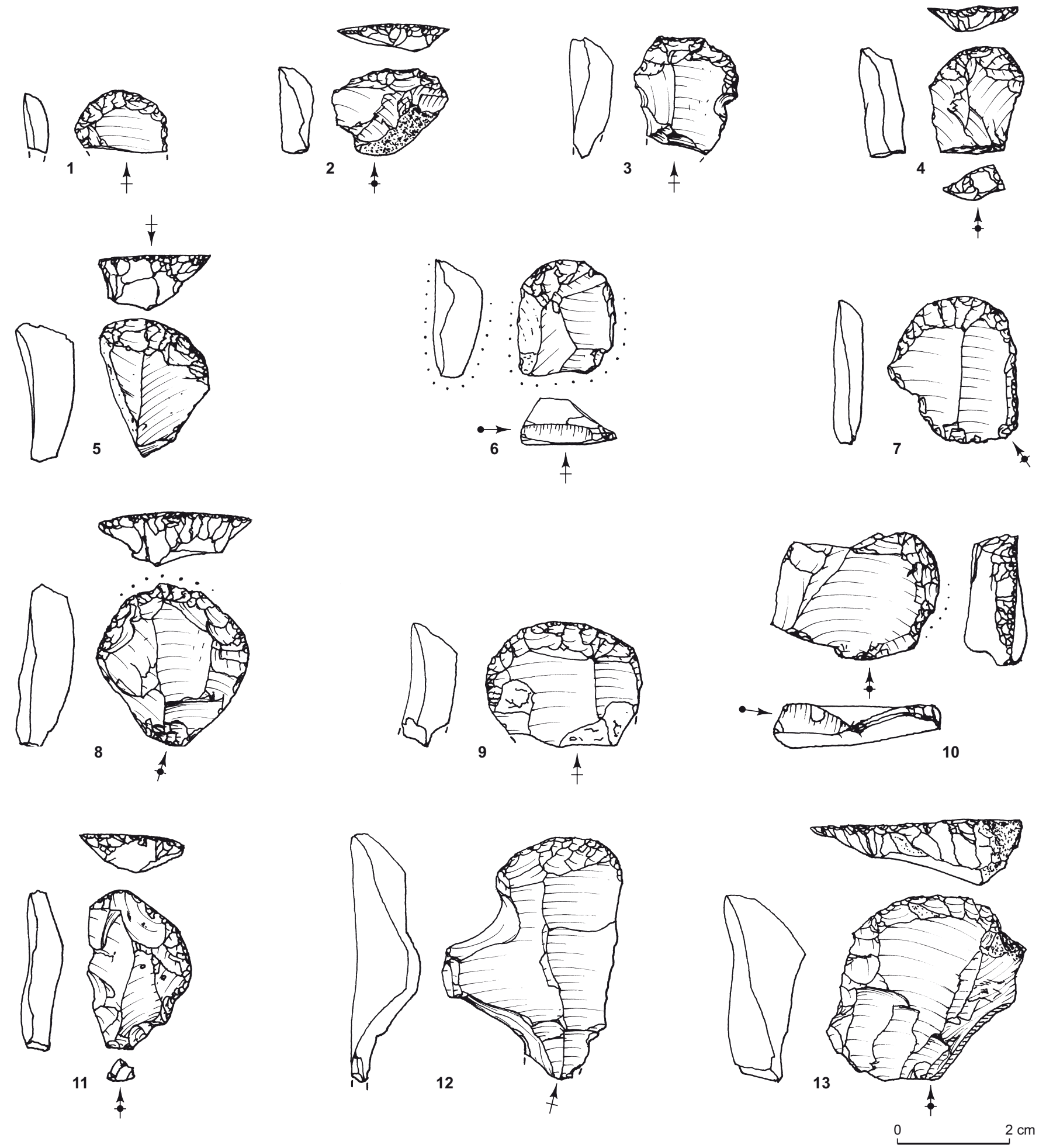

Fig. 36 - Les Chaloignes à Mozé-sur-Louet, locus 11. 1-5, 7-9, 11-13, grattoirs; 6 et 10, grattoir et burin (dessins: P. Forré, Inrap). 

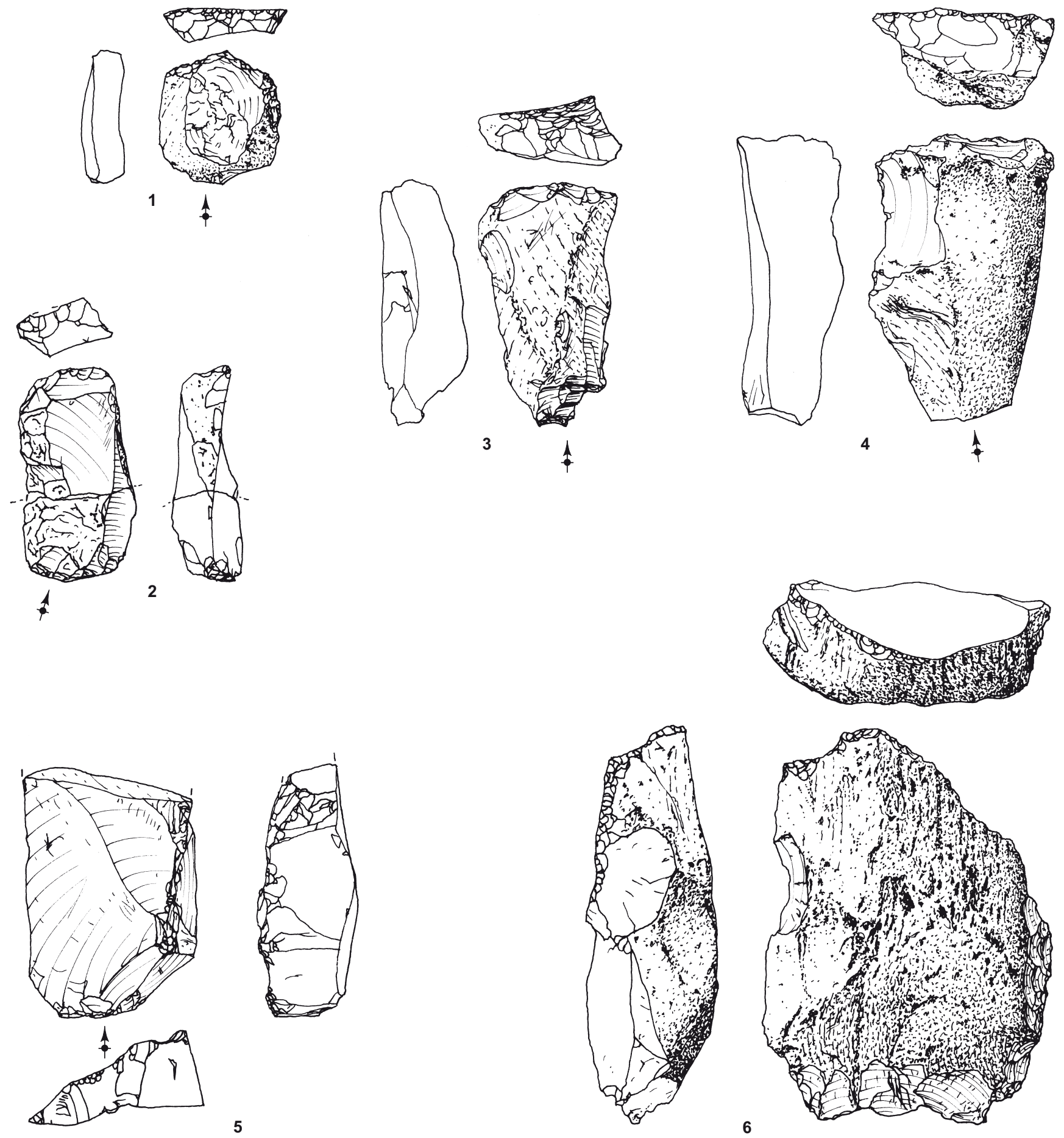

Fig. 37 - Les Chaloignes à Mozé-sur-Louet, locus 11. 1-3, grattoirs; 4, éclat à troncature transverse; 5, tablette; 6, éclat aménagé (grattoir?) (dessins: P. Forré, Inrap). 

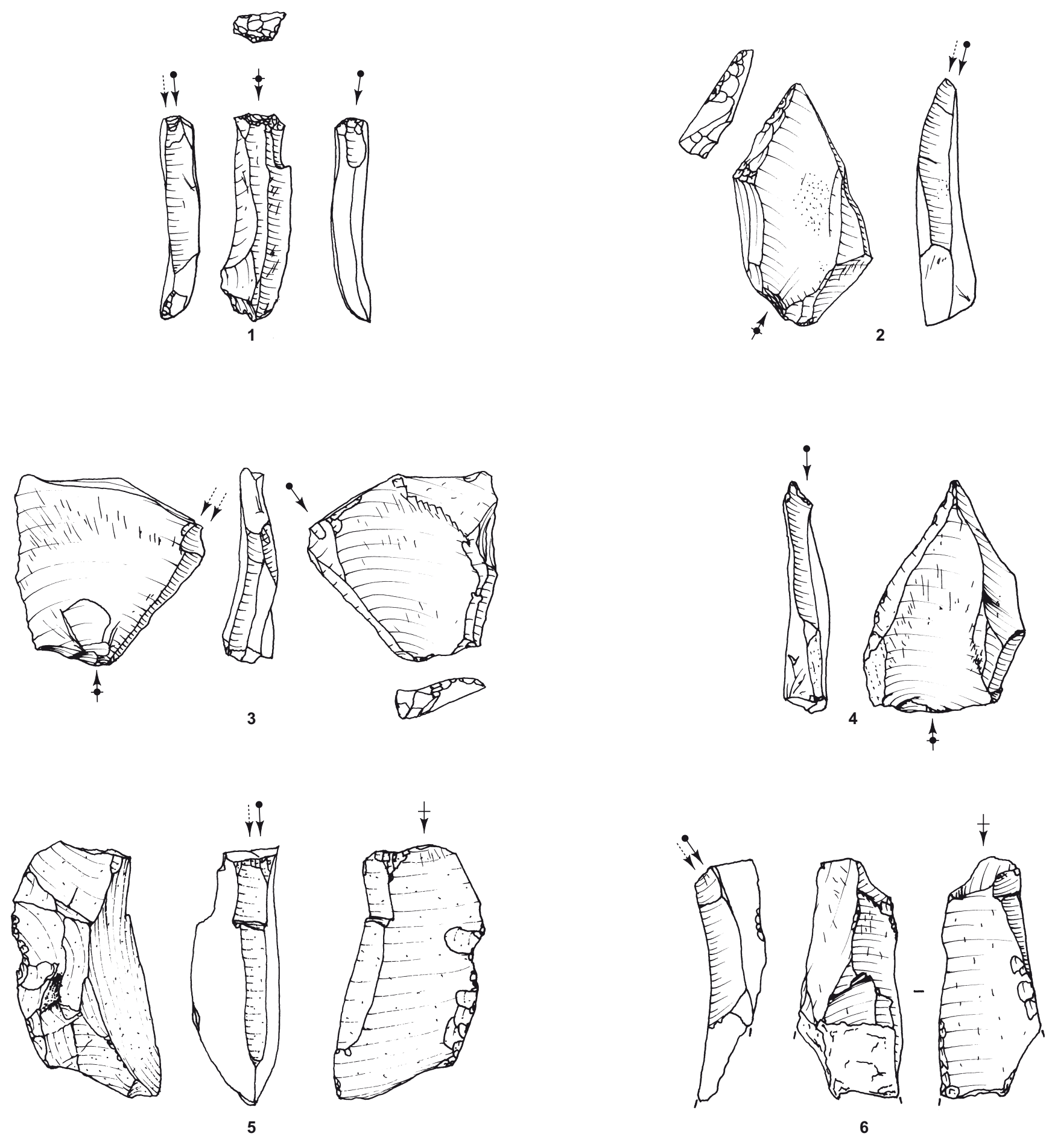
$2 \mathrm{~cm}$

Fig. 38 - Les Chaloignes à Mozé-sur-Louet, locus 11. 1-2, burins sur troncature; 3-6, burins sur cassure (dessins: P. Forré, Inrap). 
éclat tronqué à troncature concave façonné par retouches obliques. Comme on peut l'observer généralement dans l'industrie des Chaloignes, les supports de ces pièces sont des plus divers: grands ou petits, corticaux ou non, avec pourtant une exclusion certaine des produits de plein débitage. Alors que les troncatures comptent pour 9,6\% des outils, juste après les grattoirs, elles semblent toujours marginales lors de l'étude, du fait même de cet aspect médiocre et peu standardisé des supports.

Les denticulés sont anecdotiques, mais ils illustrent bien l'opportunisme dans la confection de ce type d'outils. Les deux pièces sont fabriquées sur des supports épais et très irréguliers portant encore du cortex. L'aspect denticulé du bord est obtenu par une retouche large, irrégulière et profonde (fig. 39, $\mathrm{n}^{\text {os }} 2$ et 3 ).

La transformation par un bord abattu semble répondre à la nécessité de corriger les irrégularités des supports; contrairement aux armatures ou aux grattoirs, les produits aménagés sont issus de toutes les phases des chaînes opératoires de débitage et offrent une grande hétérogénéité typologique. On remarque cependant l'épaisseur importante des produits (fig. $40, \mathrm{n}^{\text {os }} 7$ et 9 ) - autour de $10 \mathrm{~mm}$ - et la courbure du dos. On notera sur le locus 11 deux couteaux de belle facture, l'un à dos rectiligne fracturé en plus de 3 morceaux (fig. 40, no 1), l'autre entier à dos courbe, avec une ancienne tablette qui tient lieu de base retouchée (fig. $40, \mathrm{n}^{\circ} 2$ ). Les autres bords abattus sont nettement courbes. Les retouches partielles entraînent une discontinuité du dos avec certaines troncatures. Il est toujours tentant d'intégrer dans une même catégorie supposée fonctionnelle les bords abattus et les pièces ébréchées à un pan cortical. Cependant, les éclats semi-corticaux sont moins nombreux parmi les outils a posteriori que dans l'ensemble du matériel; il ne semble donc pas y avoir de production particulière d'éclats à un pan cortical ayant pu avoir la même fonction que les pièces à bord abattu.

Les éclats corticaux et semi-corticaux sont peu utilisés, alors que les lames et lamelles de plein débitage sont intensément transformées ou simplement ébréchées. Ainsi, ces lames comptent pour $8,8 \%$ de l'ensemble du matériel (débitage brut et outils), alors que leur proportion atteint 21,9\% des supports d'outils communs et 31,8\% des supports d'armatures. Les éclats simples sont utilisés à hauteur de leurs proportions dans le matériel, ce qui ne dénoterait pas de choix particuliers à destination de ces supports. Mais il faut se méfier ici du caractère unificateur de la comparaison statistique: les supports des grattoirs sont principalement des éclats à nervures régulières, dont les dimensions et les proportions laissent supposer l'existence d'une séquence de production particulière, voire d'une chaîne opératoire autonome.

Les objectifs principaux du débitage sont donc:

- des lames et lamelles larges rectilignes, assez épaisses, sans cortex, à destination des pointes,

- quelques lames transformées par des bords abattus ou au tranchant ébréché brut,

- des éclats minces, courts et larges pour les grattoirs,

- des éclats plus épais, mais au calibre non standardisé, pour le reste de l'outillage.

\section{LE DÉBITAGE}

La mise en forme n'implique pas de crête d'entame et il n'y a pas non plus d'entame sur nervures naturelles. Le nombre d'éclats corticaux est très faible. Est-ce suffisant pour supposer une ouverture des blocs hors du site? Les nombreux blocs gélifs cassés sur place laisseraient entendre le contraire.

Des schémas diacritiques réalisés sur les nucléus les plus significatifs nous laissent entrevoir la dynamique du débitage (31 nucléus entiers et fragmentés ont été étudiés). Le taux de lames et lamelles est assez faible en regard des autres locus; il atteint quand même $11,4 \%$ pour les premières et $10,0 \%$ pour les secondes. Cette production est réalisée par une méthode bipolaire sur une même table. Celle-ci est implantée dans une partie étroite et allongée du volume, un bloc ou plus rarement un éclat. Les flancs sont envahis lors de l'exploitation, même si le recul reste frontal, avec un retour systématique vers le centre de la table. La progression se fait donc par de petites séries qui reviennent constamment sur elles-mêmes. D’ordinaire, sur les nucléus laminaires observés, le dos est laissé cortical et il n’y a pas de crêtes postérieures pour l'entretien. Le large rayon de courbure, voire la rectitude, des supports d'armatures est notamment lié à cette exploitation bipolaire à alternance rapide des plans de frappe: la carène est systématiquement abaissée par les lames qui se croisent en partie distale. La rectification par des néo-crêtes est logiquement rare et se rencontre surtout sur les lames de gros modules (fig. 41, $\left.n^{\circ} 6\right)$. L'abandon définitif est lié à l'absence d'angles corrects ou à la dimension des blocs et non aux cassures ou aux rebroussés, ici assez rares. La longueur moyenne à l'abandon est la même que pour les nucléus à éclats: $43 \mathrm{~mm}$.

Un grand éclat outrepassé révèle l'existence d'une modalité différente, avec cette fois une table presque circu- 

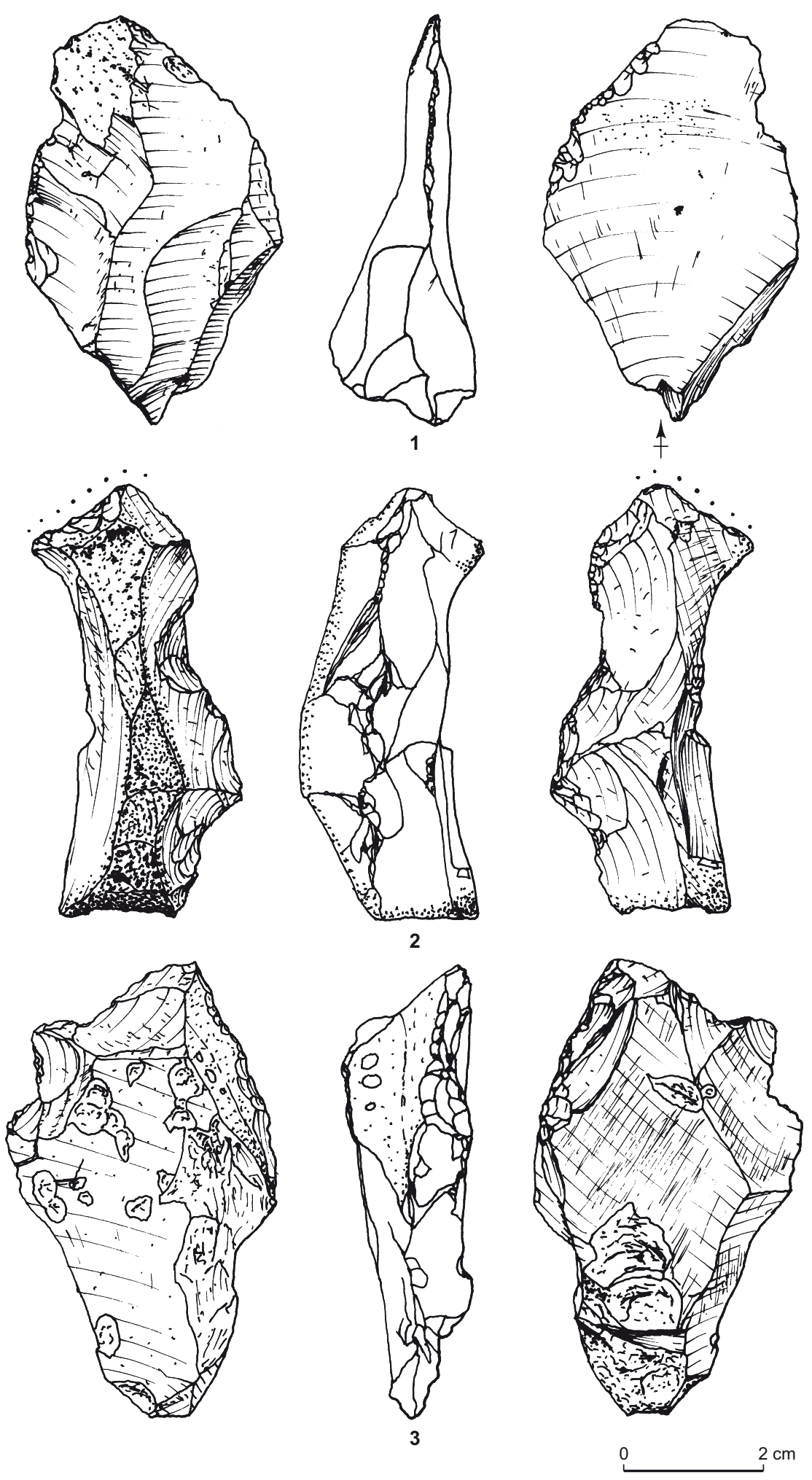

Fig. 39 - Les Chaloignes à Mozé-sur-Louet, locus 11. 1, éclat ébréché; 2 et 3, denticulés (dessins: P. Forré, Inrap). 

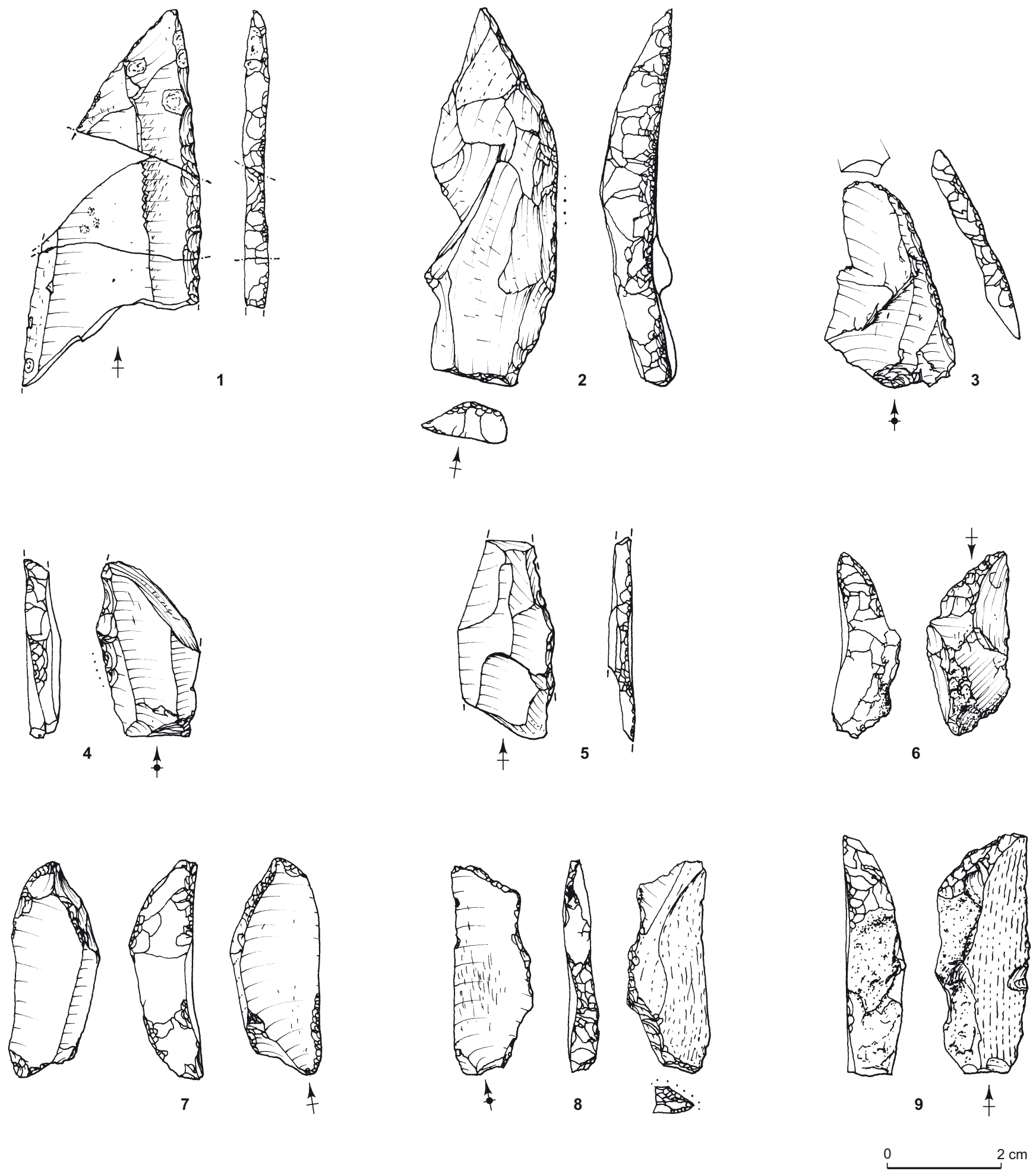

Fig. 40 - Les Chaloignes à Mozé-sur-Louet, locus 11. 1, lame à bord abattu rectiligne; 2 et 9, éclats à bord abattu courbe; 3-7, éclats à bord abattu; 8, lame à bord abattu, tronquée (dessins: P. Forré, Inrap). 

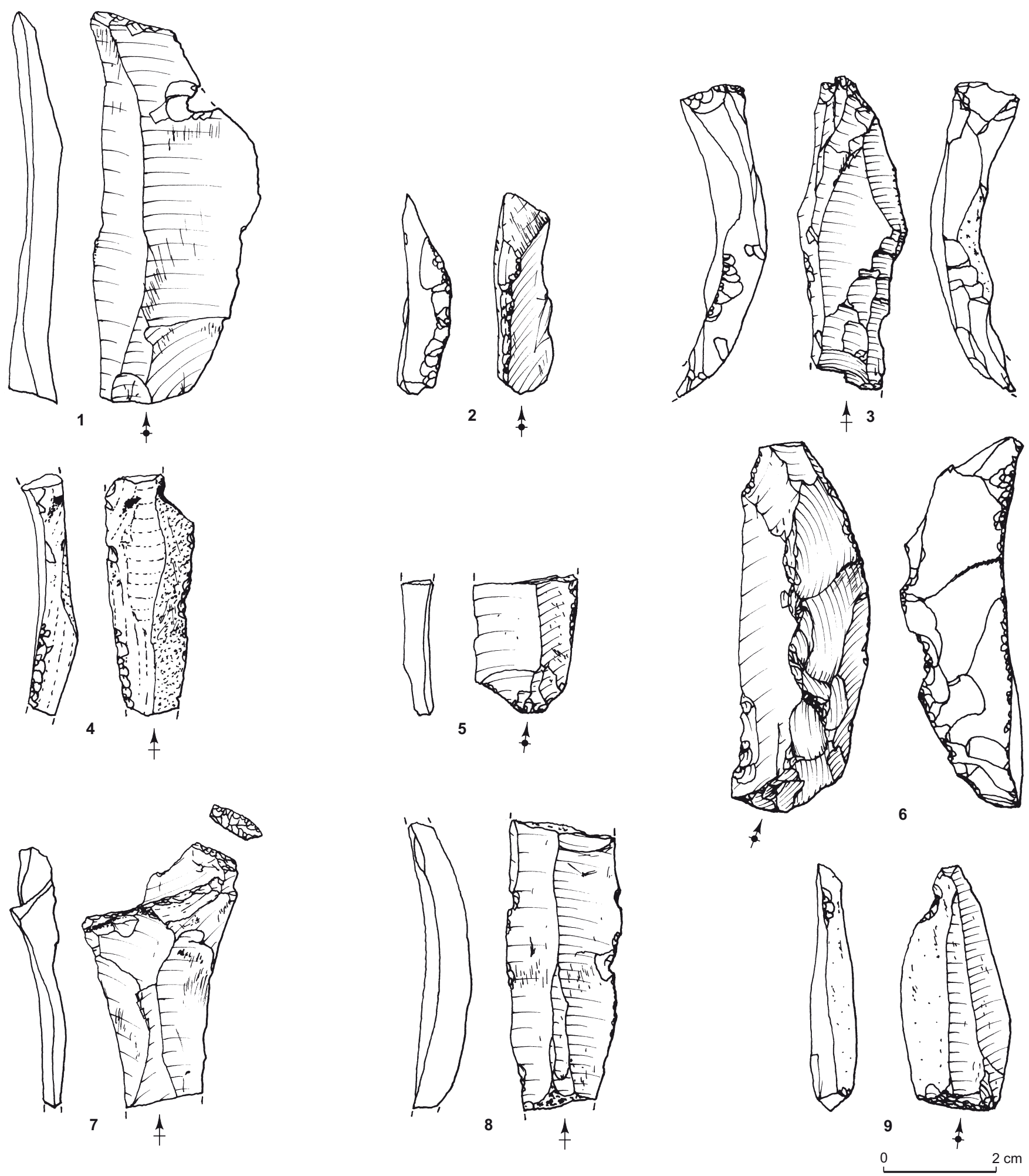

Fig. 41 - Les Chaloignes à Mozé-sur-Louet, locus 11.1 et 7, lames à troncature transverse; 2, néo-crête; 3-8, lames ébréchées (dont 6, lame à demi-crête); 9, coche sur éclat (dessins: P. Forré, Inrap). 
laire et par conséquent un nucléus à section ronde (fig. 42, $\left.n^{\circ} 4\right)$. Notons encore que, sur cette pièce, l'outrepassement est probablement volontaire dans ce débitage particulièrement soigné et unique sur le site; la carène est très peu arquée et la table montre un massif de rebroussé qui ne devait plus permettre d'assurer correctement le passage des ondes de choc. Sur les nucléus, l'angle de chasse est ouvert (entre $70^{\circ}$ et $80^{\circ}$ ). La préparation de la zone à percuter se fait comme toujours aux Chaloignes par facettage, mais celui-ci reste assez faible, puisqu'il n'affecte que $26,4 \%$ des lames de plein débitage (fig. $43, \mathrm{n}^{\circ} 1$ ) et $10,4 \%$ des éclats (la comparaison avec la moyenne générale est de peu d'intérêt ici, tant les écarts sont grands entre locus). Le taux d'abrasion sur ces deux types de produits est parmi les plus faibles de l'industrie azilienne et il s'agit toujours de ce procédé sommaire, proche de l'esquillement, observé sur le site. La technique mise en œuvre est la percussion directe dure, comme le révèle le crénelage des bords de frappe, le bulbe proéminent, les traces de martelage sur les plans de frappe, les bulbes incipients et les très nombreuses fractures en Siret. Ces données ne plaident pas en faveur d'un soin particulier apporté à cette production, même s'il reste évident que la séquence lamello-laminaire est toujours privilégiée: les talons écrasés par le percuteur ne se rencontrent pas parmi les lames de plein débitage, à la différence des éclats sans cortex ou des éclats corticaux.

Pour la production d'éclats, le débitage à partir de deux plans de frappe a encore la faveur des tailleurs, mais ils peuvent être opposés $(n=6)$ ou orthogonaux $(n=5)$. Dans les deux cas, l'implantation de la table se fait de manière à exploiter la plus grande largeur du volume et les changements d'axe sont fréquents. L'extension sur les flancs est fréquente, mais le dos est souvent laissé cortical. Les plans de frappe orthogonaux commandent soit une même table, soit deux tables différentes. La multiplication des plans de frappe peut conduire également à une morphologie globuleuse du volume $(n=5)$. Enfin, sur le locus 11 , l'identification toujours délicate du prolongement des nucléus laminaires par une production d'éclats est fondée sur de solides arguments, notamment grâce à des éclats dont la face supérieure témoigne de tables laminaires (fig. 42, $\mathrm{n}^{\circ} 2$ ).

L'architecture des nucléus n'est pas la même entre les séquences à éclats et les séquences à lames; l'implantation des tables se fait dans le premier cas sur des faces larges et dans le second cas dans la partie étroite du volume (fig. 44 et 45). La morphologie des enlèvements escomptés commande sans doute à l'orientation et à l'exploitation des blocs. En revanche, la mise en ouvre de deux plans de frappe est un caractère culturel très fort et commun à toutes les productions.

\section{FONCTIONS PRÉSENTES DANS L'AMAS}

La présence de trois galets gravés, l'un au centre et les deux autres en périphérie, en association avec le mobilier lithique, confère à ce locus une importance particulière. Pour autant, cela ne s'accompagne pas de modification dans la structure globale de l'industrie lithique, bien au contraire, puisque ce locus est le plus proche de la moyenne des locus aziliens homogènes. L'aspect général très équilibré de son outillage, le taux laminaire ou le taux de facettage ou d'abrasion assez bas, pourraient éventuellement être interprétés comme des signes d'activités domestiques par opposition avec un lieu de débitage de meilleurs tailleurs. Ce locus comporte le taux le plus fort de pièces brûlées de tous les locus aziliens $(52,6 \%)$ et un fractionnement supérieur à la moyenne. Ainsi, seulement $27,1 \%$ des pièces (outils compris) sont entières. Est-ce en rapport avec cette diversité des activités? Les regroupements de certaines matières, des armatures ou encore la proximité des éléments de raccord sont les fantômes d'activités passées, avec probablement une faible dispersion post-dépositionnelle. Ces arguments ne permettent pas de trancher entre deux hypothèses: une zone de rejets progressifs à côté d'une aire d'activité ou une zone directe d'activités variées (liée à une habitation).

\section{LE LOCUS 1: UNE ZONE DE PRODUCTION D'ARMATURES}

Le locus 1 est le premier à avoir été découvert aux Chaloignes, il se situe sur le flanc nord du vallon est (zone 1). Cette situation en périphérie de la cuvette a permis une très bonne conservation des vestiges aziliens et de leur répartition spatiale, au sommet des limons jaunes et sous $0,6 \mathrm{~m}$ de limons datés de l'Holocène. Il convient de rappeler que l'unité stratigraphique 11, qui contient le mobilier lithique, ne montre qu'un seul faciès limono-argileux, ce qui traduit une même phase de sédimentation (fig. 10). Au-dessus, une nette troncature sédimentaire a pu être mise en rapport avec la péjoration climatique du Dryas récent. Deux lignes plus ou moins parallèles, séparées de moins de $1 \mathrm{~m}$, traversaient le locus, perturbant sa partie supérieure. Il pourrait s'agir de traces de charrette d'époque historique; elles sont parallèles à un petit fossé $1 \mathrm{~m}$ plus au sud, lui-même parallèle au grand fossé d'âge historique qui drainait le vallon Est et qui scinde en deux parties le locus 2 (fig. 46). 

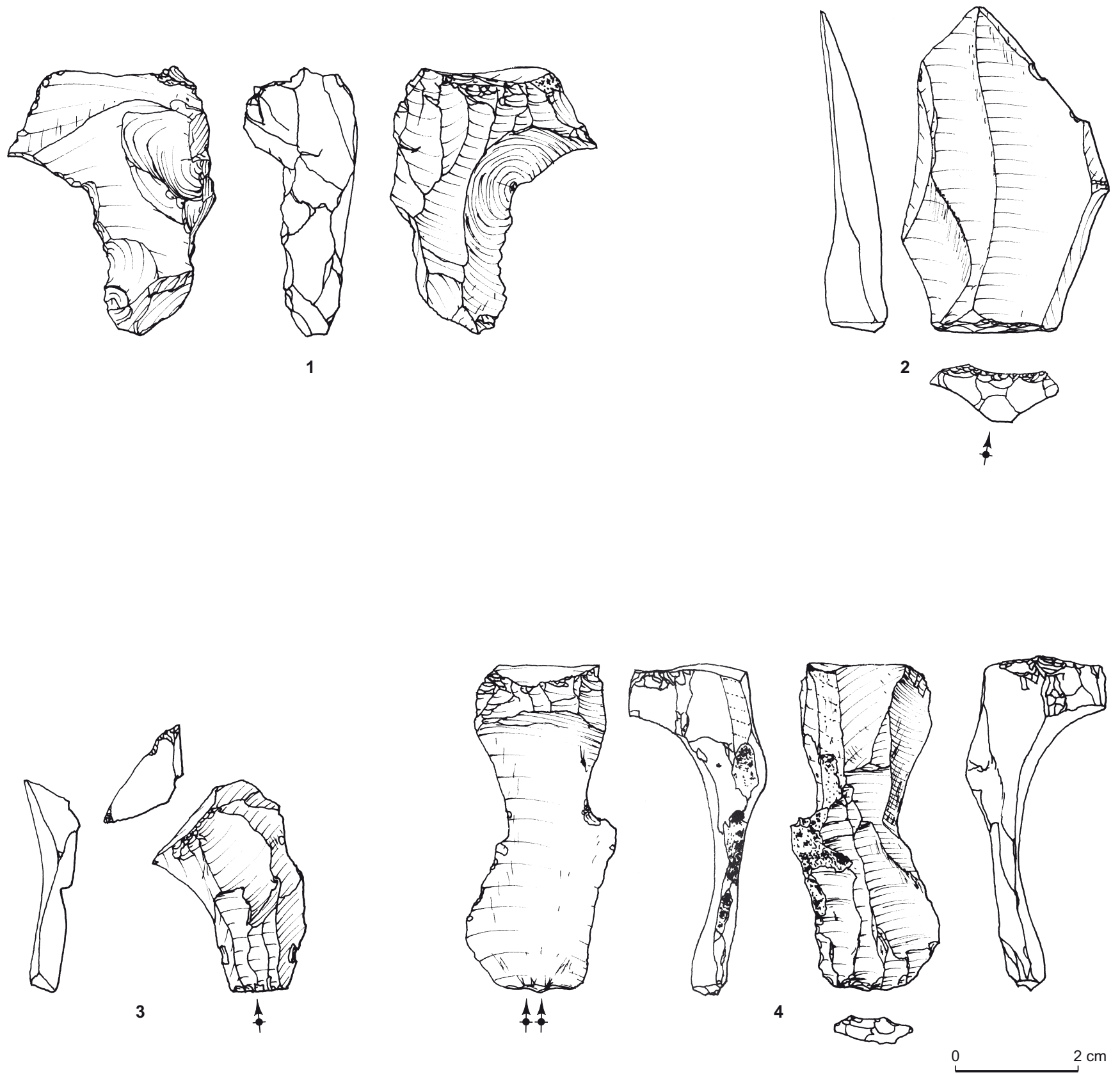

Fig. 42 - Les Chaloignes à Mozé-sur-Louet, locus 11. 1-4, éclats issus de séquence laminaire. 

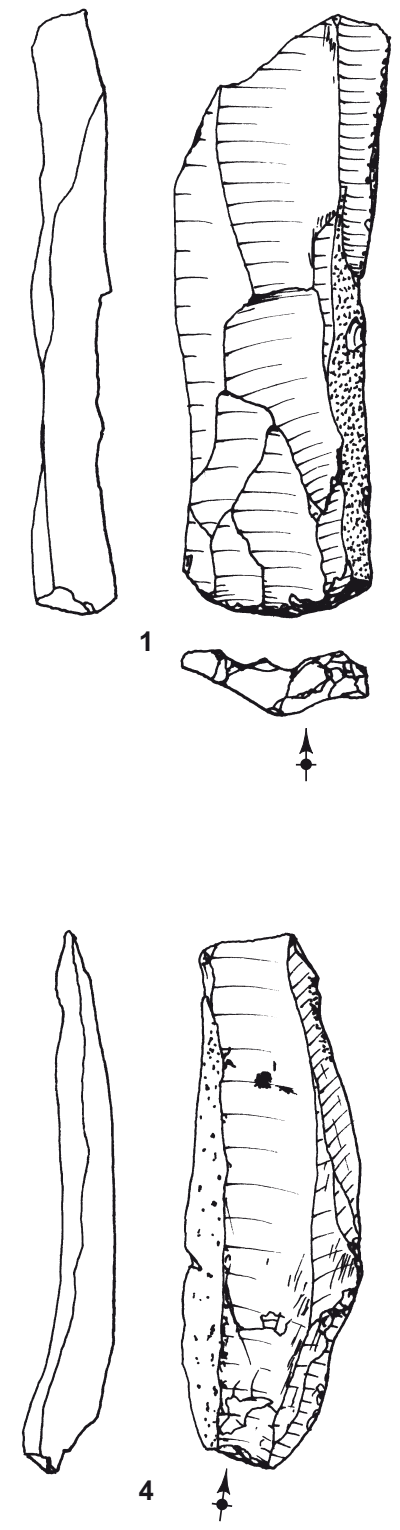
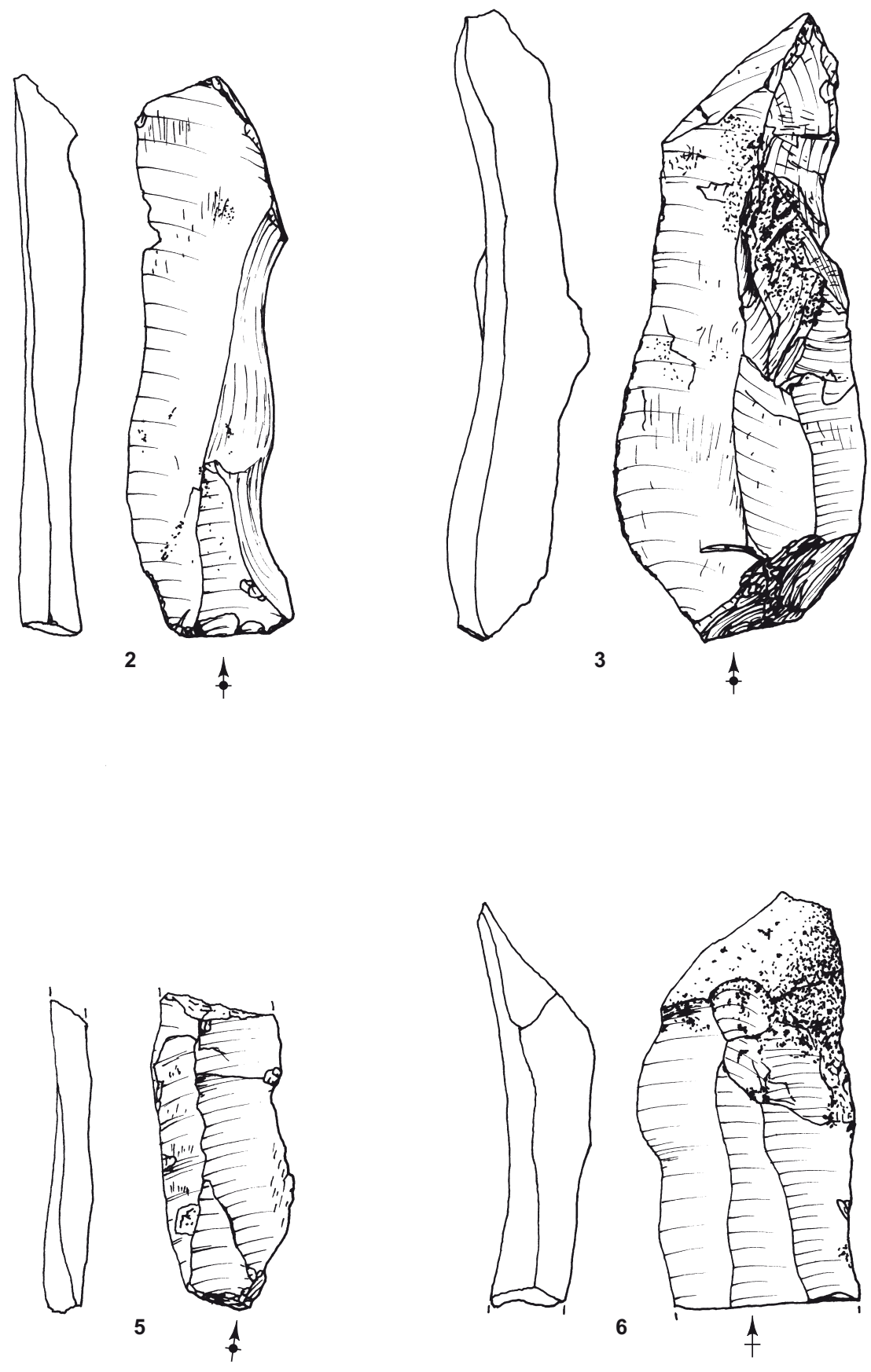
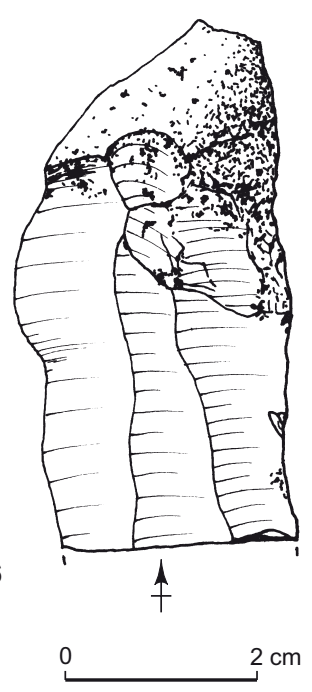

Fig. 43 - Les Chaloignes à Mozé-sur-Louet, locus 11. 1-6, lames de plein débitage ébréchées. 

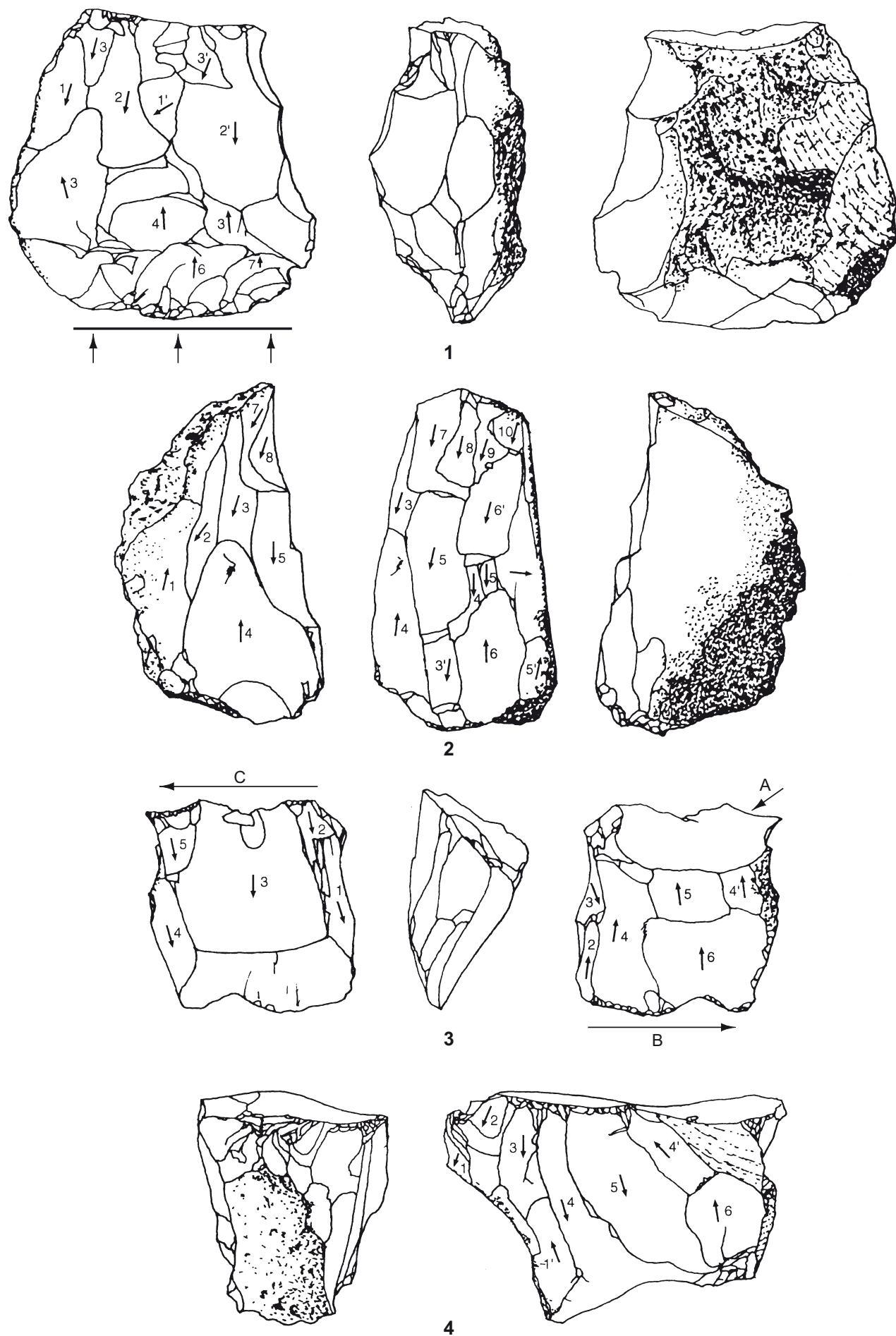

0 $2 \mathrm{~cm}$

Fig. 44 - Les Chaloignes à Mozé-sur-Louet, locus 11. Schémas diacritiques sur nucléus (dessins: P. Forré, Inrap). 

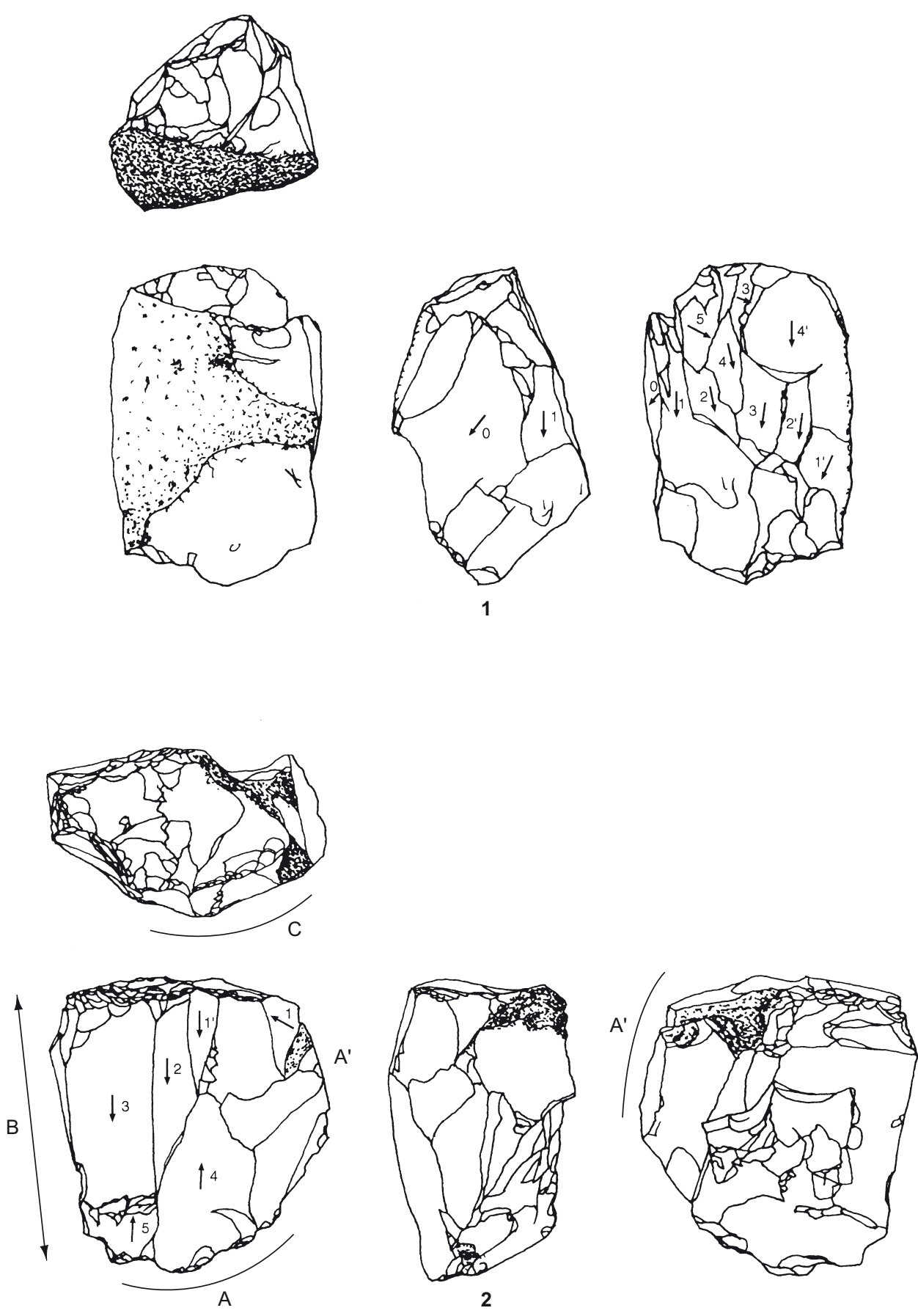

0 $2 \mathrm{~cm}$

Fig. 45 - Les Chaloignes à Mozé-sur-Louet, locus 11. Schémas diacritiques sur nucléus (dessins: P. Forré, Inrap). 


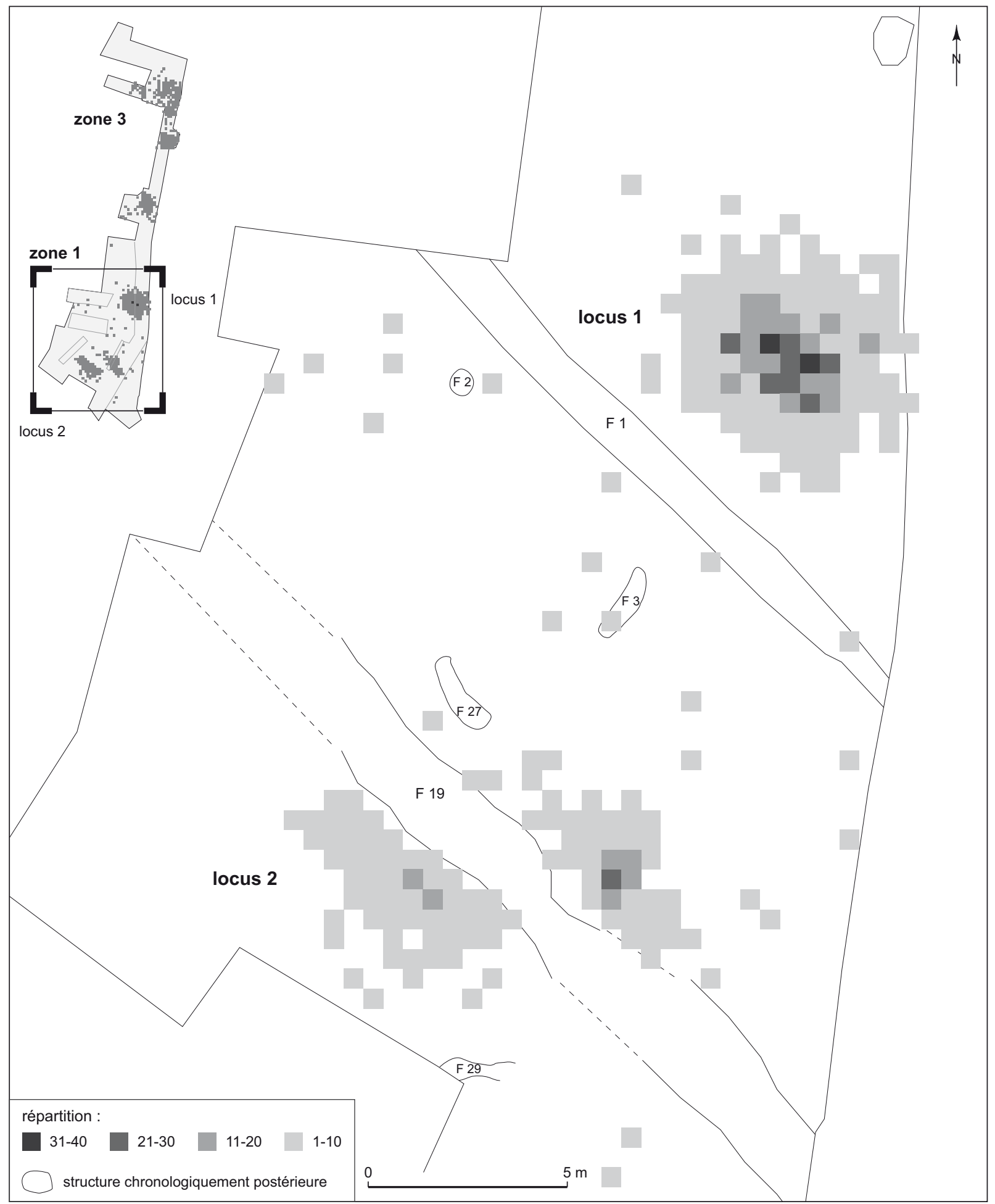

Fig. 46 - Les Chaloignes à Mozé-sur-Louet, zone 1 (locus 1 et 2), densité de mobilier par quart de $m^{2}$ (dessins: S. Sicard, Inrap et J.-F. Nauleau, Inrap). 


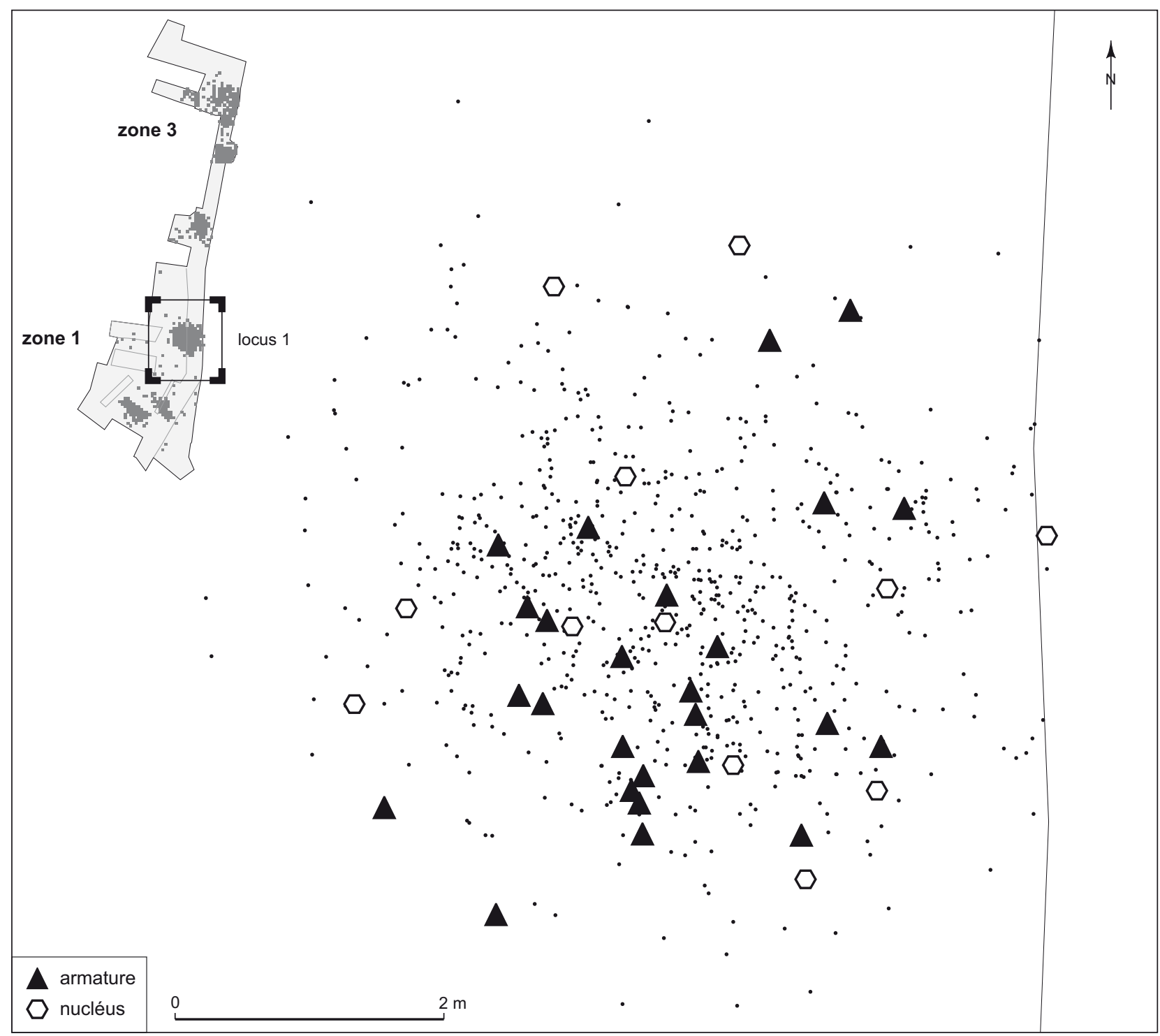

Fig. 47 - Les Chaloignes à Mozé-sur-Louet, locus 1. Plan de répartition des armatures et des nucléus (dessins: S. Sicard, Inrap et J.-F. Nauleau, Inrap).

\section{LA RÉPARTITION SPATIALE}

Ce locus comporte 819 pièces lithiques cotées, auxquelles il faut ajouter 2980 éléments, dont 2560 esquilles de moins de $10 \mathrm{~mm}$, récoltés pendant le tamisage systématique de tout le sédiment. Aucune pièce microlithique importante n'a été trouvée grâce à cette méthode, hormis un petit triangle scalène.

Une densité de vestiges plus importante apparaît au centre de cette concentration presque circulaire, sur une zone d'environ $20 \mathrm{~m}^{2}$ alors que le locus a une surface totale de plus de $30 \mathrm{~m}^{2}$ pour un diamètre de $5,50 \mathrm{~m}$. Après recoupements de multiples paramètres, une opposition apparaît entre la répartition des armatures et des nucléus, l'espace central étant dévolue aux premières tandis que les nucléus occupent la périphérie (fig. 47). Cette dichotomie nucléus/ armature a également été rencontrée dans le locus situé à 4,30 $\mathrm{m}$ plus au nord. Elle n'est pas apparemment liée à des paramètres post-dépositionnels qui auraient écarté les grosses pièces, puisque les gros éclats corticaux sont répartis uniformément. Par ailleurs, deux volumes remontés rendent compte de la dispersion des pièces d'un même débitage au 
Tabl. X-Locus 1. Répartition des outils communs suivant les matières (hors tamisage).

\begin{tabular}{|c|c|c|c|c|c|c|c|c|c|}
\hline Outil & Frac. & A & B & $E$ & Indéterminé & Montbert & Silex NR & Silex galet & Total \\
\hline Burin autre & Ent. & & & & 2 & & & & 2 \\
\hline Burin dièdre & Frag. & 1 & & & 1 & & & & 2 \\
\hline Burin / cassure & Ent. & & & & & & 1 & 1 & 2 \\
\hline Burin / troncature & Ent. & & & & & & & 1 & 1 \\
\hline Coche / lame & Frag. & & & & 1 & & & & 1 \\
\hline Éclat à troncature transverse & Ent. & & & & 1 & & & & 1 \\
\hline Grattoir + burin & Ent. & & & & 1 & & & & 1 \\
\hline Grattoir unguiforme & Ent. & & & & & & & 1 & 1 \\
\hline \multirow{2}{*}{ Grattoir / éclat } & Ent. & & & & 1 & & & & 1 \\
\hline & Frag. & & & & 1 & & 1 & & 2 \\
\hline Grattoir / lame & Frag. & & & & 2 & & & 1 & 3 \\
\hline \multirow{2}{*}{ Éclat aménagé } & Ent. & & & & 1 & & & 3 & 4 \\
\hline & Frag. & & & & 1 & & & 2 & 3 \\
\hline \multirow{2}{*}{ Éclat ébréché } & Ent. & & & & & & & 3 & 3 \\
\hline & Frag. & & 1 & 1 & 2 & 1 & & & 5 \\
\hline Lame aménagée & Frag. & & & & 1 & & & & 1 \\
\hline \multirow{2}{*}{ Lame ébréchée } & Ent. & & & & 3 & & & 2 & 5 \\
\hline & Frag. & & & & 6 & & & 1 & 7 \\
\hline Lamelle à troncature transverse & Prox. & & & & 1 & & & 1 & 2 \\
\hline \multirow{2}{*}{ Lamelle aménagée } & Ent. & & & & 1 & & & & 1 \\
\hline & Frag. & & & & 1 & & & & 1 \\
\hline Lamelle ébréchée & Frag. & & & & 4 & & & & 4 \\
\hline \multicolumn{2}{|l|}{ Total } & 1 & 1 & 1 & 31 & 1 & 2 & 16 & 53 \\
\hline
\end{tabular}

sein du locus; certaines pièces sont distantes de plus de $1 \mathrm{~m}$. Les remontages et raccords effectués sur ce locus (environ $20 \%$ de l'ensemble) donnent une idée relativement précise des chaînes opératoires représentées. Les petites séries laminaires reconstituées ici indiquent en effet que l'ensemble du débitage a été réalisé sur place. Les éléments corticaux de début de chaîne opératoire sont abondants $(12,4 \%$ du débitage). Ils correspondent certainement aux débitages d'éclats et de lames effectués sur de petits galets corticaux. Les premières lames sont corticales, il n'y a aucune mise en forme préalable du bloc. Les nucléus retrouvés sont peu nombreux; un classement des matières indique qu'au moins une dizaine de blocs ont été débités sur place. Le fait de remonter une armature au sein de la série montre que la fabrication des outils intervenait dans la continuité du débitage, sur le même espace de travail. L'abondance des esquilles de retouche vient compléter cette vision.

Nous avons déterminé deux types de locus selon le pourcentage de matériel brûlé. Le locus 1 se situe parmi ceux qui sont le plus affectés par le feu avec plus de $45 \%$ de produits brûlés. La répartition des pièces brûlées n'offre aucune structuration particulière, mais seulement une concentration au centre qui correspond à la concentration de mobilier. En l'absence de toute trace de structure de combustion à proximité, il est difficile de conclure quant à la signification du taux important de pièces brûlées. Il faut tout de même noter que les lames de plein débitage présentes dans le locus ne sont pas brûlées, alors que de nombreux blocs roulés qui n'ont pas fait l'objet d'un débitage élaboré le sont. Nous avons notamment remonté un bloc dont tous les éléments sont rubéfiés, depuis le nucléus à éclats jusqu'à l'éclat cortical d'entame. Cependant il y a divers degrés de chauffe selon les produits, qui indiquent que l'impact thermique est intervenu après le débitage.

L'industrie lithique du locus 1 du site des Chaloignes présente plusieurs caractéristiques qui dénotent une certaine variabilité inter-locus. Ainsi, la relative diversité des matières premières et leur exploitation parfois différenciée comptent parmi ces spécificités. Alors que des techniques de débitage simplifiées et peu «rentables» ont été généralement mises en œuvre, certains silex, notamment le silex E, ont fait l'objet d'une économie maximale ou bien d'une production laminaire plus élaborée, comme c'est le cas d'un silex roulé noir, témoignant d'un investissement technique plus poussé. L'originalité de ce locus réside également dans la structure de l'outillage, dominée par les armatures; l'étude suivante se consacre pour partie à comprendre ces singularités. 
Tabl. XI - Locus 1. Répartition des armatures suivant les matières (hors tamisage).

\begin{tabular}{|l|l|c|c|c|c|}
\hline \multicolumn{1}{|c|}{ Armature } & \multicolumn{1}{|c|}{ Frac. } & Montbert & Silex galet & Indét. & Total \\
\hline \multirow{2}{*}{ Monopointe azilienne } & Ent. & & & 1 & $\mathbf{1}$ \\
& Frag. & & & 4 & $\mathbf{4}$ \\
\hline \multirow{2}{*}{ Pointe azilienne } & Ent. & & & 1 & $\mathbf{1}$ \\
\cline { 2 - 6 } & Frag. & 1 & 1 & 8 & $\mathbf{1 0}$ \\
\hline $\begin{array}{l}\text { Lame étroite } \\
\text { à bord abattu }\end{array}$ & Frag. & & & 1 & $\mathbf{1}$ \\
\hline \multirow{3}{*}{ Pièce à bord abattu } & Ent. & & & 1 & $\mathbf{1}$ \\
\cline { 2 - 6 } & Frag. & & 1 & 4 & $\mathbf{5}$ \\
\cline { 2 - 7 } & Krukowski & & & 2 & $\mathbf{2}$ \\
\hline Segment & Ent. & & & 1 & $\mathbf{1}$ \\
\hline Total & & $\mathbf{1}$ & $\mathbf{2}$ & $\mathbf{2 3}$ & $\mathbf{2 6}$ \\
\hline
\end{tabular}

\section{LES MATIÈRES PREMIÈRES}

Le locus 1 est, avec les locus 4 et 12, celui qui contient le plus grand nombre de silex non roulés. Il montre aussi une grande diversité dans les sources d'approvisionnement. Nous avons pu déterminer l'exploitation de six matières premières différentes en plus des galets de la Loire (tabl. X, XI et XII). La fréquence de chacune d'entre elles est inférieure à $2 \%$ du total des matières (hors indéterminées). Certaines caractéristiques techniques nous renseignent sur l'attitude des tailleurs aziliens face à ces matières exogènes. On peut par exemple citer le silex E, pour lequel il existe un nucléus et quelques produits de débitage; ce nucléus a été abandonné en fin d'exploitation laminaire après plusieurs réorientations de la table afin de tirer parti au maximum du bloc. Les produits issus de ce nucléus sont très peu nombreux et il est probable que le nucléus était déjà mis en forme et partiellement exploité lors de son utilisation sur le locus 1. Après l'extraction de plusieurs supports destinés aux armatures, il a été laissé sur place, alors que les petites lames calibrées ont été emportées pour être utilisées sans doute après leur façonnage en pointe. Les esquilles de retouche retrouvées après le tamisage des sédiments pourraient le suggérer. Cette attitude est très rarement observée pour le débitage sur galet, où l'on ne décèle aucun souci d'économiser la matière. Au sein du silex roulé, une matière se distingue tout de même. Il s'agit d'un silex noir, fin, de bonne qualité, que l'on retrouve en très faible quantité dans les autres locus. Il a donné lieu à un débitage laminaire élaboré et poussé. Nous avons pu remonter plusieurs séries laminaires qui rendent compte de la rentabilité de cette exploitation et de l'investissement technique mis en ouvre ici. Il est intéressant de noter que le nucléus associé à ces lames n’a pas été retrouvé.
Tabl. XII - Locus 1. Décompte des éléments bruts, par catégories $d u$ débitage et matières premières (hors esquilles et tamisage).

\begin{tabular}{|c|c|c|c|c|c|c|c|c|c|}
\hline Débitage & Frac. & Roulé & B & C & D & E & $\begin{array}{c}\text { Silex } \\
\text { NR }\end{array}$ & Indéterminé & Total \\
\hline \multirow{4}{*}{ Éclat cortical } & Ent. & 35 & & & 1 & & 1 & & 37 \\
\hline & Prox. & 9 & & & & & 2 & & 11 \\
\hline & Més. & 22 & & & & & 1 & & 23 \\
\hline & Dist. & 20 & & & & & 1 & & 21 \\
\hline \multirow{4}{*}{ Éclat semi-cortical } & Ent. & 18 & & & & & 2 & & 20 \\
\hline & Prox. & 6 & & & & & 2 & & 8 \\
\hline & Més. & 8 & & & & & & & 8 \\
\hline & Dist. & 10 & & & & & & & 10 \\
\hline \multirow{4}{*}{ Éclat } & Ent. & 55 & 3 & & 1 & 2 & 1 & 74 & 136 \\
\hline & Prox. & 20 & 2 & & & 1 & 1 & 37 & 61 \\
\hline & Més. & 22 & 1 & 1 & 1 & & 3 & 63 & 91 \\
\hline & Dist. & 26 & & & & 1 & 2 & 21 & 50 \\
\hline \multirow{2}{*}{$\begin{array}{l}\text { Éclat perc. / } \\
\text { enclume }\end{array}$} & Ent. & 1 & & & & & & & 1 \\
\hline & Més. & & & & & & & 1 & 1 \\
\hline \multirow{4}{*}{$\begin{array}{l}\text { Lame de } \\
\text { plein débitage }\end{array}$} & Ent. & 3 & & & & & & 5 & 8 \\
\hline & Prox. & 17 & & & 1 & & & 26 & 44 \\
\hline & Més. & 3 & & & & & & 7 & 10 \\
\hline & Dist. & 2 & & & & & & 10 & 12 \\
\hline \multirow{4}{*}{ Lame autre } & Ent. & 5 & & & 1 & & 1 & 2 & 9 \\
\hline & Prox. & 6 & & & & & & & 6 \\
\hline & Més. & 9 & & & & & 1 & 1 & 11 \\
\hline & Dist. & 2 & & & & & & & 2 \\
\hline \multirow{4}{*}{$\begin{array}{l}\text { Lamelle de } \\
\text { plein débitage }\end{array}$} & Ent. & 3 & & & & & & 7 & 10 \\
\hline & Prox. & 3 & & & 1 & & & 22 & 26 \\
\hline & Més. & 4 & & & & & & 15 & 19 \\
\hline & Dist. & 4 & & & & 2 & & 6 & 12 \\
\hline \multirow{4}{*}{ Lamelle autre } & Ent. & 5 & & & & & & 4 & 9 \\
\hline & Prox. & 5 & & & & & & 1 & 6 \\
\hline & Més. & 4 & & & & & & 2 & 6 \\
\hline & Dist. & 4 & & & & & & & 4 \\
\hline \multirow{2}{*}{ Nucléus à éclat } & Ent. & 2 & & & & & & 1 & 3 \\
\hline & Frag. & 1 & & & & & & 1 & 2 \\
\hline \multirow{2}{*}{ Nucléus à lame } & Ent. & 1 & & & & 1 & & 1 & 3 \\
\hline & Frag. & 2 & & & & & & & 2 \\
\hline $\begin{array}{l}\text { Nucléus } \\
\text { indéterminé }\end{array}$ & Frag. & 2 & & & & & & & 2 \\
\hline \multirow{4}{*}{ Tablette } & Ent. & 2 & & & & & & & 2 \\
\hline & Prox. & 1 & & & & & & 1 & 2 \\
\hline & Més. & 1 & & & & & & 1 & 2 \\
\hline & Dist. & 0 & & & & & & 1 & 1 \\
\hline \multirow{4}{*}{ Néo-crête } & Ent. & 1 & & & & & & 1 & 2 \\
\hline & Prox. & 1 & & & & & & 1 & 2 \\
\hline & Més. & 1 & & & & & & 2 & 3 \\
\hline & Dist. & 1 & & & & & & 2 & 3 \\
\hline \multirow{2}{*}{ Chute de burin } & Ent. & 0 & & & & & & 1 & 1 \\
\hline & Més. & 0 & & & & & & 1 & 1 \\
\hline Casson & & 17 & & & & & 1 & 18 & 36 \\
\hline \multicolumn{2}{|l|}{ Total } & 364 & 6 & 1 & 6 & 7 & 19 & 336 & 739 \\
\hline
\end{tabular}

\section{LES OUTILS}

Le taux d'armatures est très élevé avec $33 \%$ de la totalité de l'outillage aménagé contre moins de $11 \%$ pour tous les autres locus. Il s'agit de pointes aziliennes, apparemment 

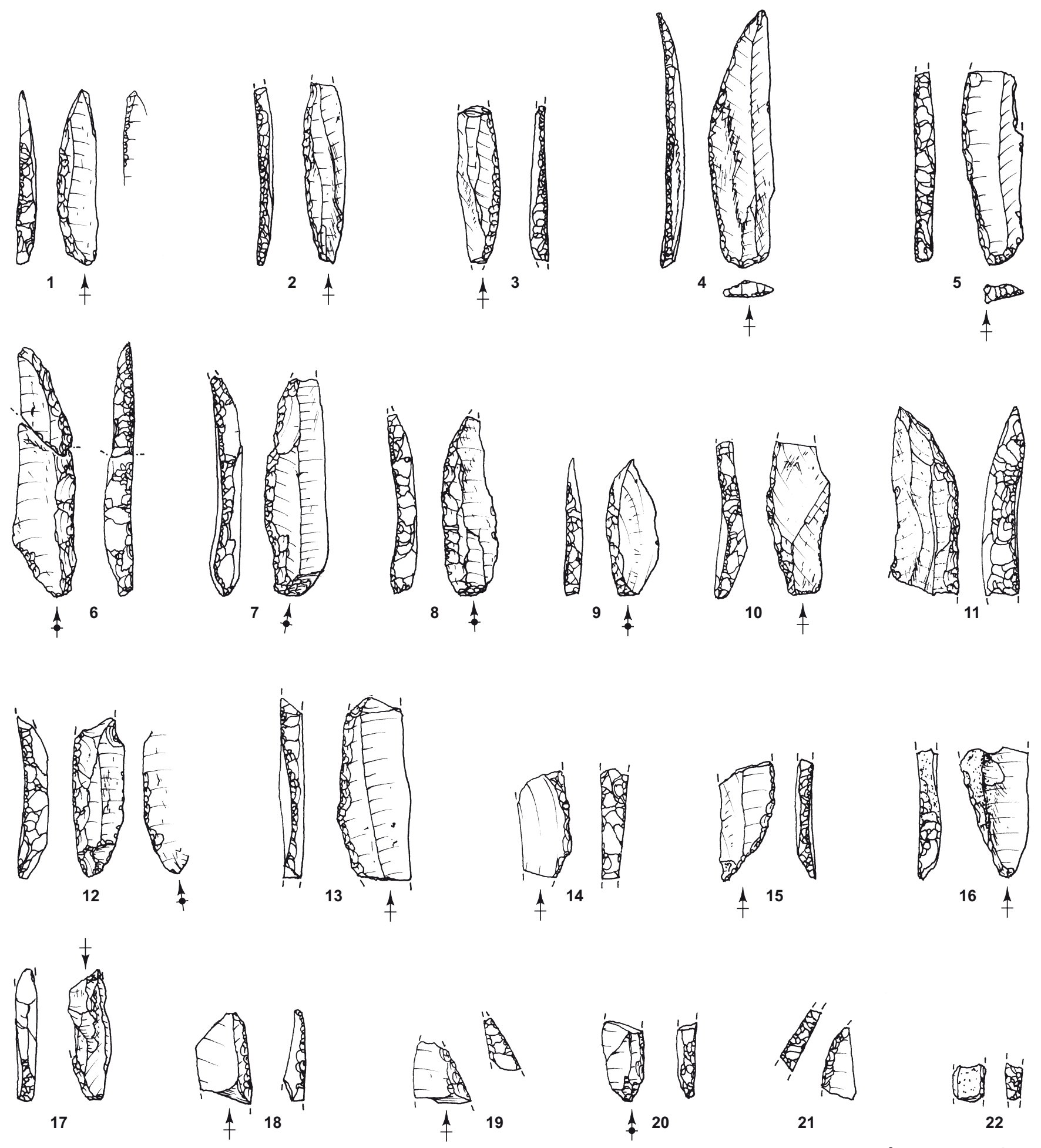

Fig. 48 - Les Chaloignes à Mozé-sur-Louet, locus 1. 1-3, 6-7, 11, 13-16, pointes aziliennes; 4 et 5, pointes de Malaurie; 8-10, 12, monopointes aziliennes; 17-22, pièces à bord abattu (dessins: P. Forré, Inrap). 
toutes des monopointes très fragmentées. Sur ce locus, la base n'est jamais rétrécie par retouches du bord opposé au dos, mais ce rétrécissement est présent par la seule morphologie du support. L'extrémité apicale de ces pièces correspond le plus souvent à la partie distale du support. Il est important de noter la présence de deux pointes aziliennes à base transverse retouchée, assimilables à des pointes de Malaurie (fig. 48, $\mathrm{n}^{\text {os }} 4$ et 5). Ces types récents dans l'évolution de l'Azilien sont rares sur le site. L'ensemble de ces armatures présente des supports de dimensions très variées. On peut distinguer des pointes «macrolithiques» (d'après la distinction établie dans Fagnart, 1997) réalisées sur lame qui forment le groupe des pointes aziliennes, et des pièces à bord abattu qui sont réalisées sur lamelles et constituent un ensemble plus hétérogène qui recouvre peut-être des pièces différentes d'un point de vue fonctionnel. Les supports des pointes aziliennes sont des lames régulières à deux pans, à profil rectiligne ou légèrement arqué en partie distale. Une unique lame présente du cortex sur un pan, mais il a été partiellement supprimé par la retouche du bord ( $\left.n^{\circ} 16\right)$. Seules deux armatures ont été réalisées sur des éclats dont l'épaisseur est identique à celle des lames. La latéralisation des pointes est légèrement plus marquée à gauche, mais les proportions pour chacun des bords ne sont pas significatives. Les dos des pointes sont arqués, généralement bien courbes mais présentent parfois un certain angle $\left(n^{\text {os }} 7,10\right.$ et 13$)$, très insuffisant pour les qualifier de pointe de Creswell. Enfin, un fragment distal de pointe a une épaisseur qui dénote au sein des armatures ( $\left.n^{\circ} 11\right)$. Parmi les éléments de moins de $10 \mathrm{~mm}$ récoltés lors du tamisage, trois pièces à dos à fracture Krukowski et trois cassures orthogonales sur lames étroites viennent confirmer un façonnage sur place des pièces à bord abattu. En revanche, les fragments apicaux de pointes n'ont pas été détectés, alors même qu'ils manquent sur de nombreuses armatures de ce locus: sans ossements, ce n'est pas tout à fait suffisant pour poser l'hypothèse d'une zone de réfection d'armes de chasse et non de traitement des carcasses. C'est donc la convergence des données recueillies lors de la fouille et lors du tamisage qui doit être mise en avant par cette étude, plus que les résultats intrinsèques des éléments du tamisage.

Il convient aussi de mentionner deux armatures attribuables au Mésolithique (fig. 49, $\mathrm{n}^{\text {os }} 6$ et 7 ). La première est un segment entier, trouvé au sommet de la nappe de vestiges lithiques, dans l'US 11. Il porte un piquant-trièdre qui atteste du procédé du microburin pour la transformation du support. La retouche est croisée et la corde est bordée. Le second est un triangle scalène, cassé en partie proximale, issu du tamisage. Il est allongé, à trois cotés retouchés avec une base formée par une troncature oblique, rectiligne. Leur présence dans ce locus reflète celle d'une composante mésolithique sur l'ensemble du site dont la localisation précise n'a pu être établie. Ces deux pièces sont isolées et nous n'avons décelé aucune autre intrusion mésolithique dans le locus. À l'inverse, le taux d'outils communs est faible, puisqu'il ne représente que $6,5 \%$ de l'ensemble des produits. Les grattoirs, avec $10,1 \%$ du total des outils, sont plus nombreux que les burins $(8,9 \%)$. Il y a un grattoir caréné réalisé sur une lame très régulière (fig. 49, no 13 ), qui se différencie des autres grattoirs courts et peu épais. Leurs fronts sont convexes, souvent unguiformes (fig. $49, \mathrm{n}^{\text {os }} 9,10$ ). Les burins dièdres $(3,7 \%)$ et les burins sur cassure $(3,7 \%)$ prédominent, alors que les burins sur troncature sont minoritaires. La variété du reste de l'outillage est faible en comparaison des autres locus, puisqu'il y a seulement quelques troncatures et des éclats retouchés qui accompagnent les grattoirs et les burins. Il n'y a pas de grandes lames à dos comme on peut en rencontrer dans d'autres locus. Les éclats aménagés et ébréchés sont les mieux représentés (28,3\%). Enfin, on peut souligner la présence de quelques outils particuliers. Il y a un grattoir, dont le front est presque rectiligne, opposé à un burin dièdre (fig. 50, $\mathrm{n}^{\mathrm{o}} 2$ ). Deux lamelles régulières sont tronquées en partie distale (fig. 51, $\mathrm{n}^{\text {os }} 4$ et 6 ). Étant donné le peu d'outillage lamellaire rencontré sur le site, on ne peut exclure qu'il s'agisse de troncatures spontanées obtenues au moment du détachement de la lamelle. Enfin, il existe un éclat à troncature très concave (fig. 51, no 1 ) qui dégage deux extrémités apicales que l'on peut interpréter comme des perçoirs.

\section{LE DÉBITAGE LAMINAIRE}

Le caractère laminaire de ce locus est bien marqué, puisque lames et lamelles représentent 26,3\% des supports (hors outillage) et $31,1 \%$ des supports si l'on se base sur le NMI (produits entiers et proximaux, bruts et retouchés). Il faut noter la part importante des lamelles dans ce total. Les objectifs du débitage sont orientés vers la production de supports laminaires peu calibrés mais réguliers, destinés à la fabrication d'armatures pour la réfection d'outils de chasse. L'histogramme des largeurs de produits bruts diffère quelque peu de celui du locus 11 . On constate une sous-représentation des produits entre $11 \mathrm{~mm}$ et $12 \mathrm{~mm}$, autour de $14 \mathrm{~mm}$ et entre $16 \mathrm{~mm}$ et $17 \mathrm{~mm}$ (fig. 52). Au contraire entre $7 \mathrm{~mm}$ et $10 \mathrm{~mm}$, les lamelles sont nom- 

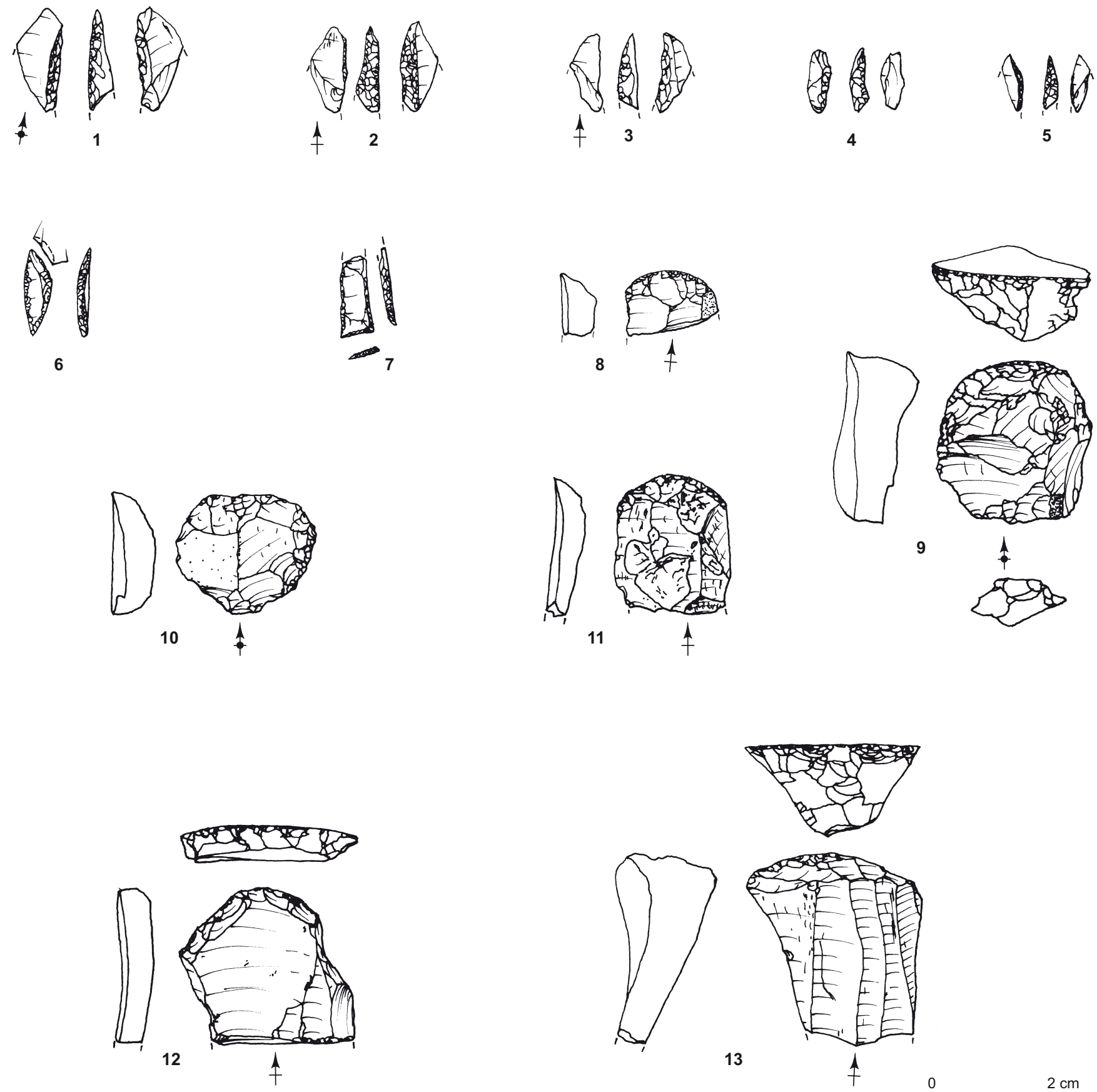

0 $2 \mathrm{~cm}$

Fig. 49 - Les Chaloignes à Mozé-sur-Louet, locus 1. 1-5, pièces à bord abattu; 6, segment; 7, triangle scalène; 8-13, grattoirs (dessins: P. Forré, Inrap). 

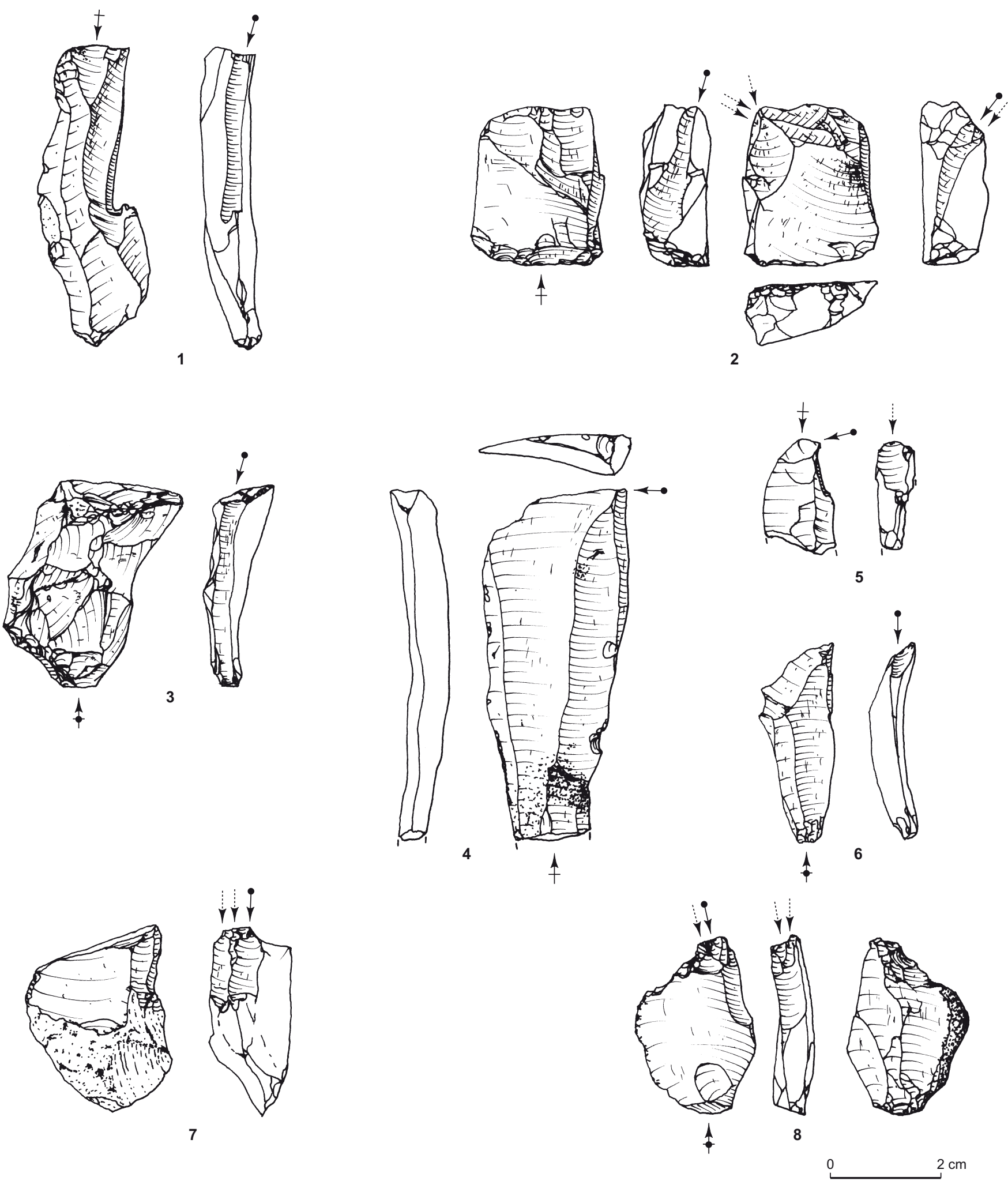

Fig. 50 - Les Chaloignes à Mozé-sur-Louet, locus 1. 1, 3, 6, burins; 2, grattoir et burin; 4 et 5, burins dièdres; 7 et 8 , burins sur troncature (dessins: P. Forré, Inrap). 

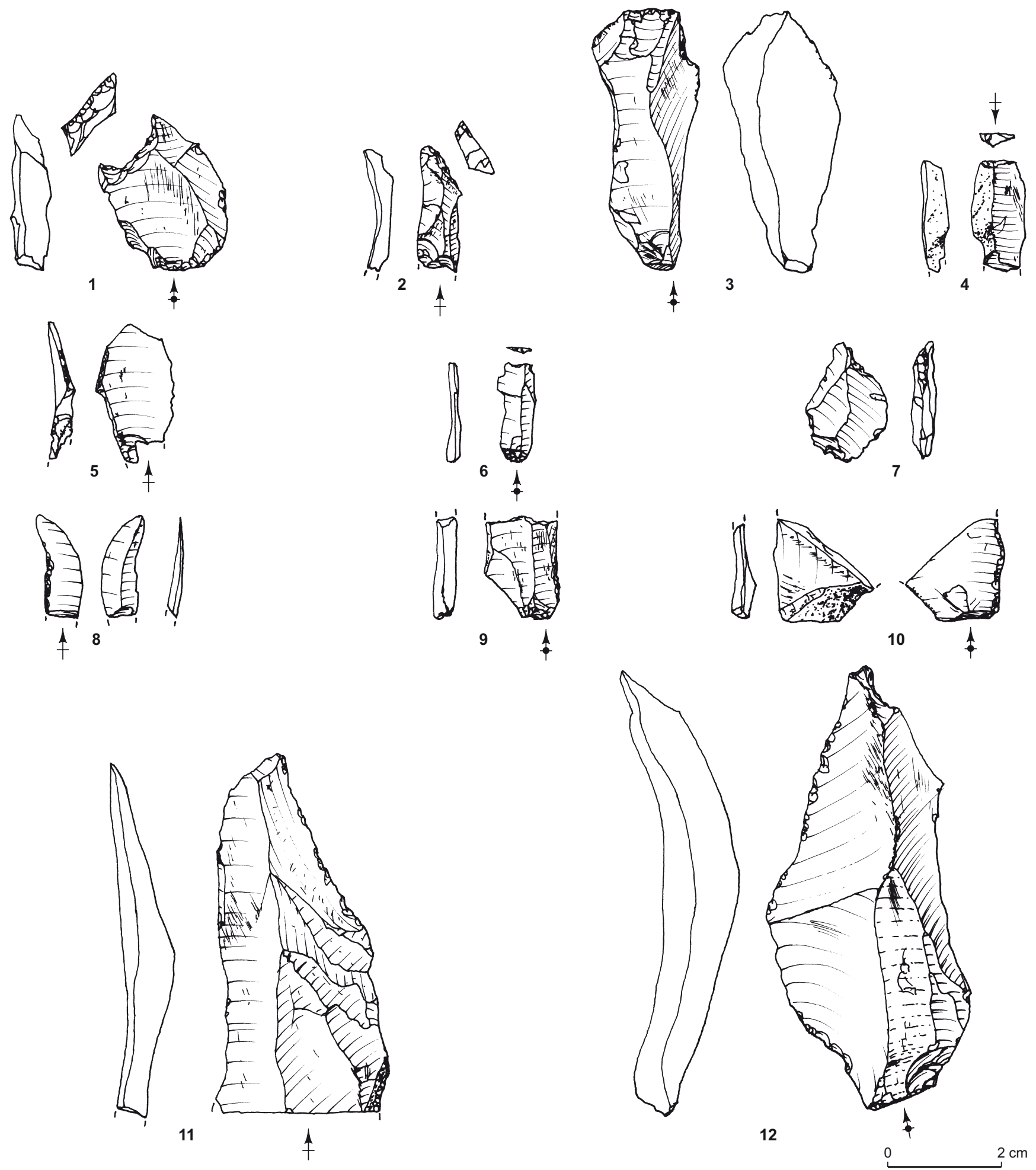

Fig. 51 - Les Chaloignes à Mozé-sur-Louet, locus 1. 1, éclat à troncature transverse (perçoir ?); 2, lamelle à troncature oblique; 3, 5, 7, 10, éclats aménagés ou utilisés; 4 et 6, lamelles à troncature transverse; 8, lamelle ébréchée; 9, 11 et 12, lames ébréchées (dessins: P. Forré, Inrap). 


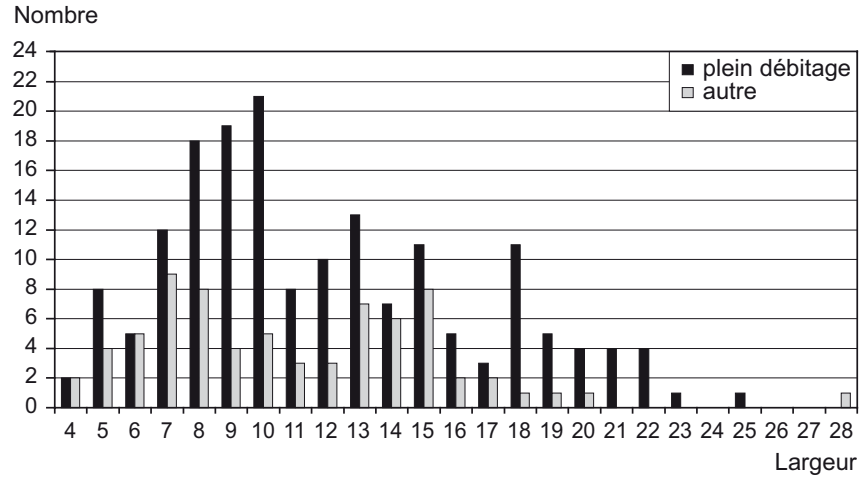

Fig. 52 - Les Chaloignes à Mozé-sur-Louet, locus 1. Diagramme des largeurs de lames et lamelles (en $\mathrm{mm}$ ).

breuses. Faut-il voir dans ces carences le prélèvement de supports de plus grande largeur? Une telle répartition reflète peut-être un emport de pièces laminaires, dont les largeurs seraient comprises entre $11 \mathrm{~mm}$ et $17 \mathrm{~mm}$. Cette dispersion illustre encore une fois la faible calibration des supports, perceptible sur l'ensemble du site. En revanche, l'absence des valeurs 11-12 mm est bien marquée et correspondrait après aménagement aux largeurs minimales des armatures (qui descendent rarement sous les 7-8 mm). La longueur des lames et lamelles brutes n'a pas pu être étudiée, en raison de la grande fragmentation du matériel; aucune des lames de plein débitage n'est entière. Le type de cassure n'indique cependant pas de réduction volontaire de ces supports et ce taux est très proche de la moyenne pour l'ensemble des locus (65\% de fragmentation pour l'ensemble du débitage).

Les séries laminaires remontées correspondent souvent aux premières phases du débitage ou à des extensions des tables sur les flancs, les lames semi-corticales étant les plus aisées à assembler. Plusieurs séries laminaires de plein débitage ont quand même pu être remontées, ainsi qu'une pointe azilienne insérée dans une des séries. Cette pointe a été cassée pendant la fabrication du dos et elle a donc été rejetée. Il y a seulement trois nucléus laminaires en fin d'exploitation contre sept à éclats. Il est intéressant de souligner l'absence de nucléus laminaire correspondant aux séquences laminaires remontées dans le locus; les matières sont d'ailleurs fort différentes. On constate l'inverse dans le locus 2, où il y a quatre nucléus laminaires alors que les lames sont presque inexistantes. Ces nucléus du locus 1 ont soit été emportés hors du locus afin de servir à une production ultérieure, soit transformés pour une ultime production d'éclats. Les supports des nucléus sont des blocs roulés ou des cassons. L'implantation de la table concerne toujours le côté le plus long du bloc; l'étroitesse de la table n'est pas un critère discriminant. Une des modalités observées correspond à un rythme semi-tournant avec un envahissement d'un flanc et ensuite un recul par séquences obliques, de part et d'autre de la jonction des deux tables. L'exploitation des deux surfaces peut se faire par un ou deux plans de frappe. Un des remontages laminaires pourrait correspondre à une telle conception volumétrique. Ces deux surfaces de débitage ne couvrent qu'un tiers de la circonférence; les dos n'ont pas été mis en forme et restent le plus souvent corticaux. Une autre modalité - ou éventuellement une évolution de la précédente? - correspond à une exploitation bipolaire d'une table large avec un rythme frontal, les flancs étant presque inexistants.

Les plans de frappe forment des angles ouverts avec les tables, entre $70^{\circ}$ et $90^{\circ}$. L'enlèvement de deux tablettes en cours de séquences laminaires est attesté par un remontage, avec un recul d'environ $10 \mathrm{~mm}$ entre les deux réfections du plan de frappe, soit deux ou trois épaisseurs de lames. L'entretien du cintre est assuré par le recul oblique de la table, sur une face étroite et une face large ou dans le cas d'un débitage sur table large par des enlèvements d'axe. Les séquences laminaires débordantes remontées le prouvent, de même que certains éclats débordants. Le contrôle de l'angle de la carène est assuré par le second plan de frappe et plus rarement par des néo-crêtes partielles. Le soin apporté au débitage des supports laminaires se manifeste aussi par le taux de facettage très important des talons des lames de plein débitage $(31,1 \%$, tabl. XIII). L'abrasion est également importante avec plus de $62 \%$ des lames abrasées, mais aussi $43 \%$ des éclats. Les derniers enlèvements laminaires ont une longueur d'au moins $30 \mathrm{~mm}$, ce qui correspond bien à la taille minimale recherchée pour les supports d'armatures.

\section{LE DÉBITAGE D'ÉCLAT}

Les nucléus à éclats sont peu porteurs d'informations techniques, mais ils permettent de mettre en évidence une chaîne opératoire d'éclats sur petits blocs, distincte de celle des lames. La conduite du débitage se fait selon le même schéma, à partir d'un plan de frappe, plus rarement de deux, sur la face large du nucléus, avec une exploitation s'étendant sur un flanc du volume, exceptionnellement sur les deux. Un éclat a aussi servi de support; la table est alors implantée en partie distale de l'éclat, sur la face inférieure. À cause d'une prédétermination assez faible, seuls quelques éclats ont été débités avant le rejet des nucléus. 
Tabl. XIII - Locus 1. Proportions de talons des principaux produits bruts ou retouchés.

\begin{tabular}{|l|c|r|r|r|r|r|c|c|c|}
\hline \multicolumn{1}{|c|}{ Débitage } & Cortical & Dièdre & Ecrasé & Facetté & Filiforme & Large lisse & Mince lisse & Punctiforme & Total \\
\hline Éclat & 5,9 & 3,4 & 10,8 & 9,4 & 9,4 & 13,8 & 25,6 & 21,7 & $\mathbf{1 0 0 , 0}$ \\
\hline Éclat cortical & \multicolumn{1}{|c|}{28,0} & 2,0 & 14,0 & 4,0 & 4,0 & 18,0 & 20,0 & 10,0 & $\mathbf{1 0 0 , 0}$ \\
\hline Éclat perc. / enclume & 0,0 & 0,0 & 100,0 & 0,0 & 0,0 & 0,0 & 0,0 & 0,0 & $\mathbf{1 0 0 , 0}$ \\
\hline Éclat semi-cortical & 3,3 & 6,7 & 23,3 & 3,3 & 0,0 & 33,3 & 20,0 & 10,0 & $\mathbf{1 0 0 , 0}$ \\
\hline Lame autre & 5,3 & 10,5 & 5,3 & 10,5 & 0,0 & 0,0 & 52,6 & 15,8 & $\mathbf{1 0 0 , 0}$ \\
\hline Lame de plein débitage & 4,9 & 1,6 & 3,3 & 31,1 & 3,3 & 8,2 & 34,4 & 13,1 & $\mathbf{1 0 0 , 0}$ \\
\hline Lamelle autre & 0,0 & 0,0 & 13,3 & 6,7 & 13,3 & 0,0 & 26,7 & 40,0 & $\mathbf{1 0 0 , 0}$ \\
\hline Lamelle de plein débitage & 2,4 & 2,4 & 7,3 & 17,1 & 7,3 & 9,8 & 36,6 & 17,1 & $\mathbf{1 0 0 , 0}$ \\
\hline Néo-crête & 0,0 & 0,0 & 0,0 & 0,0 & 0,0 & 25,0 & 75,0 & 0,0 & $\mathbf{1 0 0 , 0}$ \\
\hline Tablette & 0,0 & 0,0 & 20,0 & 20,0 & 0,0 & 20,0 & 40,0 & 0,0 & $\mathbf{1 0 0 , 0}$ \\
\hline Total (en \%) & $\mathbf{7 , 5}$ & $\mathbf{3 , 3}$ & $\mathbf{1 0 , 7}$ & $\mathbf{1 2 , 1}$ & $\mathbf{6 , 5}$ & $\mathbf{1 3 , 5}$ & $\mathbf{2 8 , 7}$ & $\mathbf{1 7 , 7}$ & $\mathbf{1 0 0 , 0}$ \\
\hline
\end{tabular}

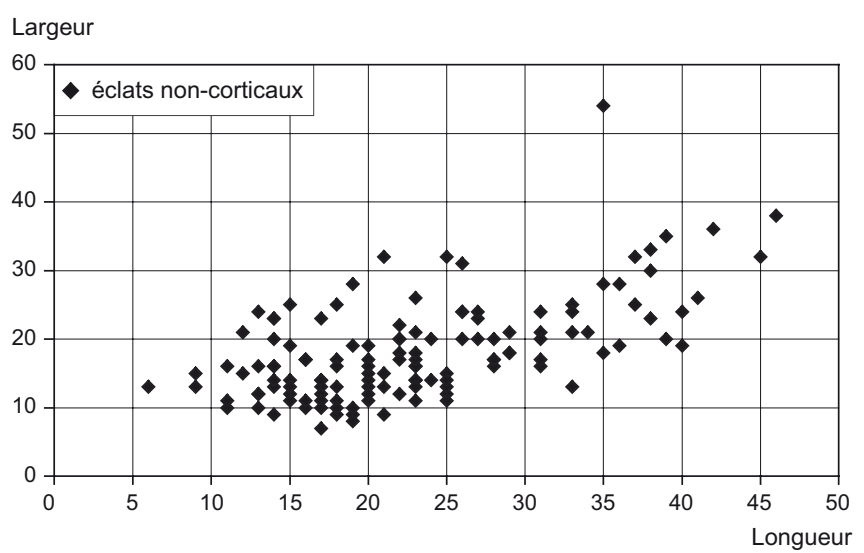

Fig. 53 - Les Chaloignes à Mozé-sur-Louet, locus 1. Longueur/largeur des éclats non-corticaux (en mm).

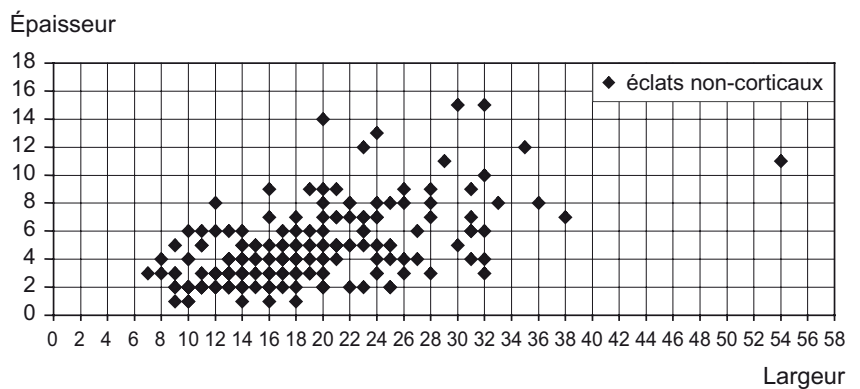

Fig. 54 - Les Chaloignes à Mozé-sur-Louet, locus 1. Largeur/épaisseur des éclats non-corticaux (en mm).

Ces derniers ne présentent ni accident lié au débitage, ni épuisement de la matière qui justifient leur abandon, mais plutôt une absence d'angulation correcte. Il s'agit d'une attitude opportuniste, avec une exploitation rapide de petits blocs voués à une utilisation immédiate. Les éclats présentent aussi des caractéristiques récurrentes intéressantes. Nous avons déjà mis en évidence sur le site l'existence d'une courte séquence opératoire d'éclats qui intervient sur de petits blocs différenciés. Sur le locus 1, cette recherche d'éclats est manifeste. La représentation des longueurs et largeurs des éclats non-corticaux montre une similitude morphométrique des produits, dont la longueur mesure environ $20 \mathrm{~mm}$ et la largeur entre $10 \mathrm{~mm}$ et $15 \mathrm{~mm}$ (fig. 53 et 54). Il y a cependant une corrélation moyenne entre ces deux données (coefficient de corrélation de 0,601), de même qu'entre la largeur et l'épaisseur $(0,592)$. Elles sont à comparer aux coefficients obtenus pour les éclats corticaux, nettement plus élevés (longueur et largeur de 0,727; largeur et épaisseur de 0,708). En d'autres termes, on peut conclure que les corrélations dimensionnelles au sein des éclats de plein débitage ne sont pas déterminantes pour démontrer la standardisation stricte de la production.

On constate donc deux attitudes différentes face aux nucléus de ce locus, non pas tant dans la conduite des opérations que dans les fins de chaînes. La recherche de petites lames et le soin apporté à leur obtention est manifeste, tant sur les produits que sur les nucléus. Pour cela, de petits nucléus préformés ou rapidement mis en forme sur cette zone ont été utilisés afin de fabriquer des pointes aziliennes servant pour les armes de chasse. Après la confection de ces supports, les nucléus laminaires ont été emportés ailleurs s'ils pouvaient encore servir, ou bien ils ont été rejetés sur place s'ils étaient épuisés. Des petits galets corticaux ont servi au débitage d'éclats. Ces derniers ont été obtenus selon les mêmes principes techniques que les lames, mais il n'y a pas eu de souci de rentabilité et les nucléus ont été abandonnés très vite après satisfaction des besoins. La question des ponts entre ces deux séquences reste posée; la reconversion de certains nucléus laminaires pour d'ultimes 
Tabl. XIV - Locus 1. Décompte du matériel analysé en tracéologie et répartition des différentes utilisations.

\begin{tabular}{|c|c|c|c|c|c|c|c|c|}
\hline \multirow{2}{*}{ Type de pièce } & \multirow{2}{*}{$\begin{array}{l}\text { Nombre } \\
\text { observé }\end{array}$} & \multirow{2}{*}{$\begin{array}{c}\text { Nombre } \\
\text { avec trace }\end{array}$} & \multirow{2}{*}{$\begin{array}{l}\text { Nombre de } \\
\text { bords actifs }\end{array}$} & \multicolumn{5}{|c|}{ Matière d'oeuvre } \\
\hline & & & & Projectile & Peau & Végétal & Peau sèche ou végétal & $?$ \\
\hline Grattoir & 6 & 4 & 4 & & 4 & & & \\
\hline Grattoir / burin & 1 & & & & & & & \\
\hline Burin & 2 & 1 & 1 & & & 1 & & \\
\hline Troncature & 3 & 1 & 2 & & & 1 & & 1 \\
\hline Lame aménagée & 3 & & & & & & & \\
\hline Eclat ébréché & 3 & & & & & & & \\
\hline Lame ébréchée & 2 & 2 & 3 & & & 1 & 2 & \\
\hline Lamelle ébréchée & 1 & & & & & & & \\
\hline Eclat brut & 19 & & & & & & & \\
\hline Lame brute & 18 & & & & & & & \\
\hline Lamelle brute & 12 & & & & & & & \\
\hline Chute de burin & 3 & & & & & & & \\
\hline Pointe & 16 & 4 & 4 & 3 & 1 & & & \\
\hline Pièce à bord abattu & 10 & 1 & 1 & 1 & & & & \\
\hline Scalène & 1 & 1 & 1 & 1 & & & & \\
\hline Total & 100 & 14 & 16 & 5 & 5 & 3 & 2 & 1 \\
\hline
\end{tabular}

productions d'éclats est possible, comme l'indiqueraient quelques éclats à nervures laminaires.

\section{APPROCHE DU FONGTIONNEMENT DU LOCUS À L'AIDE D'UNE ÉTUDE TRACÉOLOGIQUE}

Le but de cette première étude tracéologique est d'approcher les comportements techno-fonctionnels des Aziliens de l'Ouest, non seulement en identifiant les modes d'utilisation des principaux types d'outils mais aussi en tentant de comprendre les objectifs du débitage, en particulier la production des petits éclats et lamelles qui ne constituent pas des supports d'outils aménagés. Ces analyses visent également à déterminer les cycles d'utilisation, éventuellement distincts, pour les différentes catégories de débitage et de matières premières.

\section{Le matériel analysé}

Le matériel sélectionné pour l'analyse préliminaire comprend une centaine de pièces dont 15 outils du fonds commun, 27 armatures et 58 produits bruts, incluant les supports ébréchés (tabl. XIV). L'état de conservation de la collection s'est avéré satisfaisant. Seulement $27 \%$ des pièces sont altérées, principalement par l'action du feu, plus rarement par des phénomènes de patine. D'autres processus ont provoqué la formation de «bruit de fond» de traces parasites. Des dégradations macroscopiques de la surface des silex, se développant sous forme de petites zones très brillantes proches de lustrés, ont parfois perturbé la lecture des microtraces. Ces altérations ont réellement compromis l'analyse de 11 pièces: un grattoir, un burin, une lame aménagée, un éclat ébréché, six pièces brutes et une pointe azilienne. Elles ont pu aussi contrarier la lecture des micropolis les plus ténus, comme peuvent l'être ceux des matières carnées tendres. Toutefois, elles n'expliquent que partiellement le faible taux d'utilisation de l'outillage.

\section{Gestion des outillages}

La faiblesse relative de ce taux (14\%) est surtout révélatrice des comportements techno-fonctionnels des Aziliens des Chaloignes. Les outillages sont globalement peu exploités, les usages peu intenses et les utilisations multiples rares. Ainsi, on ne compte que deux outils employés sur plusieurs tranchants (un éclat tronqué et une lame ébréchée). Les produits bruts (et ébréchés), qui composent plus de la moitié de l'échantillon, sont très rarement impliqués dans des activités, seuls deux supports sur 58 ont livré des stigmates d'usage. Pour les pièces retouchées, outils du fonds commun et armatures, les proportions sont plus élevées (respectivement $40 \%$ et $23 \%$ ), mais n'ont qu'une valeur indicative en raison des effectifs considérés.

\section{Les armatures}

Parmi les seize pointes aziliennes examinées, quatre livrent des stigmates interprétables. L'usage en élément de projectile est clairement attesté sur trois d'entre elles qui présentent des fractures symptomatiques d'impact, pseudo- 
burinantes, en flexion et/ou scalariformes, dans la zone apicale/mésiale (fig. $48, \mathrm{n}^{\text {os }} 5,7,14$ ). Sur une de ces pointes, une pointe de Malaurie (fig. 48, n 5 ), la valeur diagnostique de ces macro-traumatismes est confirmée par des microtraces d'impact. Des zones linéaires d'abrasion à stries larges et superficielles, issues de la fracture apicale, se développent parallèlement à l'axe longitudinal de l'armature. Pour ces trois armatures, la localisation et l'orientation des stigmates confirment une insertion à l'extrémité de la hampe de flèche et un mode de fonctionnement en élément perçant.

Les traces observées sur la deuxième pointe de Malaurie sont plus ambiguës (fig. 48, nº 4). Elles résultent d'un usage sensiblement différent, vraisemblablement d'un travail de découpe. Le fil du tranchant porte des micro-ébréchures trapézoïdales ou triangulaires obliques et bifaciales dues à une cinématique longitudinale unidirectionnelle effectuée dans le sens basal/apical. Un léger émoussé du fil et des petites zones de micropoli sont préservés entre les ébréchures. Le poli rugueux et mat peut se rapporter à une matière carnée résistante telle que de la peau, plutôt sèche. L'usage de cette pointe en élément de couteau est donc très probable. Toutefois, il reste difficile de dire s'il s'agit de l'emploi «opportuniste» d'une pointe de projectile ou de l'illustration de la variabilité fonctionnelle des armatures. Les deux cas de figure sont connus en contexte azilien, à la Balma de la Margineda (Andorre), par exemple, où une seule pointe à dos courbe a découpé une matière indéterminée (Philibert, 2002) ou bien sur le site d'Andernach (Rhénanie-Palatinat, Allemagne) où des pointes aziliennes ont été utilisées en éléments de couteaux pour la découpe de tissus carnés (Plisson, 1985). La variabilité observée parmi les pointes de Rekem révèle des comportements qui ne sont pas strictement comparables. En effet sur ce site, une sélection des armatures a été pratiquée selon leur largeur. Jusqu'à $12 \mathrm{~mm}$, les pointes sont utilisées en éléments de projectile $(n=27)$, et, au-delà de ces dimensions, elles servent comme des couteaux de boucherie $(n=5)$ (Caspar, De Bie, 1996). Il existe donc deux populations d'armatures qui s'excluent morphométriquement et fonctionnellement, ce qui ne semble pas le cas aux Chaloignes puisque les différentes utilisations sont observées sur un même type de pointe.

Restituer la nature de l'éventuelle variabilité fonctionnelle des pointes des Chaloignes demandera des analyses complémentaires. En l'état actuel des données, le caractère unique de cette pointe de Malaurie utilisée pour couper plaide en faveur d'un usage occasionnel ou opportuniste, inhérent à la capacité d'adaptation de ces derniers chasseurs dont les outillages fournissent de multiples témoignages. Aux Chaloignes, comme dans la plupart des assemblages ayant fait l'objet d'une étude tracéologique, les pointes ont donc, dans leur grande majorité, fonctionné en éléments de projectile et ont servi d'armatures perçantes. Cette univocité typo-fonctionnelle est reconnue dans de nombreux contextes chronoculturels et, notamment durant l'Épipaléolithique, au Pont d'Ambon (Bourdeilles, Dordogne; Célérier, Moss, 1983), à Saint-Antoine (Vitrolles, Hautes-Alpes; Gagnepain et al., 1999), à Saint-Thibaud-de-Couz (Savoie; Philibert, 1995), sur les sites de la Margineda (Andorre), l'Abeurador (Hérault), Buholoup (Haute-Garonne) (Philibert, 2002) ou bien encore sur le site de Berniollo en Espagne (Gonzalez Urquijo, Ibanez Estévez, 1993). Cette spécialisation des pointes s'observe indépendamment de leur morphologie. Sur le site des Chaloignes, les dimensions des armatures analysées varient sensiblement, de $3 \mathrm{~cm}$ à $4,5 \mathrm{~cm}$ pour les longueurs, de $0,7 \mathrm{~cm}$ à $1,2 \mathrm{~cm}$ pour les largeurs et de $0,2 \mathrm{~cm}$ à $0,5 \mathrm{~cm}$ pour les épaisseurs. La variabilité morphométrique des pointes tirées (avec trace d'impact) suggère la diversité des types de flèches composant les «carquois » épipaléolithiques. Les pièces à bord abattu analysées offrent, dans la plupart des cas, des fractures peu diagnostiques et sont généralement vierges de microtraces d'utilisation. Toutefois, un fragment basal a permis l'observation d'une zone d'abrasion striée qui se développe parallèlement à l'axe longitudinal sur la zone de fracture (fig. 48, $\mathrm{n}^{\circ} 6$ ). Ces microstigmates d'impact indiquent que ce fragment constitue une partie basale de pointe de projectile et que les pièces à bord abattu appartiennent, au moins pour une partie d'entre-elles, à la population des pointes à dos. Enfin, le triangle scalène à petit coté court, probablement mésolithique, montre une fracture apicale scalariforme et des enlèvements bifaciaux symptomatiques d'un impact longitudinal pouvant arguer en faveur d'un fonctionnement en élément perçant et d'une insertion par la petite base. Ce mode de fonctionnement, qui n'est certes pas le plus fréquent, est néanmoins attesté, à Téviec (Morbihan) par exemple où un microlithe de même type a été retrouvé fiché dans une vertèbre (Péquart et al., 1937), mais aussi en domaine sauveterrien à Fontfaurès (Lentillac-Lauzès, Lot; Philibert, 1991), l'Abeurador (Hérault) ou au Pas de la Charmate (Isère; Philibert, 2002).

\section{L'outillage commun}

Vingt et un outils du fonds commun ont été portés à l'étude. Parmi les six grattoirs, quatre présentent des altérations de surface dues à l'action du feu ou à des phénomènes 
de désilicification. Les indices fonctionnels sont toutefois visibles sur quatre pièces. Les bords actifs de ces pièces sont constitués des fronts retouchés convexes, dont les fils portent de légers émoussés et des micropolis attribuables au raclage des peaux (fig. $49, \mathrm{n}^{\text {os }} 8,10,11,12$ ). En raison des dégradations de surface, l'état physique de la peau n'a pu être déterminé, sauf dans un cas où il s'agit de peau reverdie (fig. $49, \mathrm{n}^{\mathrm{o}} 12$ ). On note un grattoir abandonné après ravivage $\mathrm{du}$ front.

L'un des deux burins examinés semble avoir servi sur le tranchant inférieur du pan du burin latéral (fig. 50, no 4). Les micro-ébréchures régulières perpendiculaires au fil, de morphologie écailleuse à scalariforme, le micro-émoussé résiduel et le poli bien que dégradé, peuvent être attribués, à titre d'hypothèse, au raclage ou au rabotage d'un végétal ligneux comme le bois. Le second burin, très altéré par le feu, n'a pas autorisé la lecture de trace d'utilisation.

Un éclat tronqué, morphologiquement proche d'une pièce à coche distale, est l'un des rares outils présentant plusieurs bords actifs (fig. 51, n 1 ). Sur la troncature transverse, un fin liseré de poli brillant résulte d'une utilisation probable, mais non certaine, pour le raclage de bois ou d'une autre matière végétale rigide. La délinéation concave du bord actif permet d'envisager un travail de régularisation d'une branche au faible diamètre, d'un fût ou d'une hampe. Sur le bord droit, la micro-usure se rattache à une cinématique longitudinale, mais la matière d'œuvre, vraisemblablement tendre à mi-dure, n'a pu être clairement identifiée. Aucune des trois lames aménagées étudiées n'a fourni de donnée fonctionnelle.

\section{Les produits de débitage}

Dans la catégorie des outils ébréchés, deux grandes lames ont livré des traces d'utilisation. Sur une première lame (fig. 51, $\mathrm{n}^{\circ} 11$ ), une micro-altération de surface a perturbé la lecture des traces. Quelques stries, parfois désordonnées, le plus souvent fines et parallèles au bord, affectent les deux tranchants et témoignent d'un usage probable sur les bords droit et gauche dont les fils portent un fort micro-émoussé. Le poli, mat et légèrement rugueux, est attribuable à un travail de découpe sur une matière résistante tendre à mi-dure. Il pourrait s'agir de peau sèche ou d'un végétal ligneux. La deuxième lame (fig. 51, no 12), offre un important micro-émoussé sur une zone à délinéation légèrement convexe du bord gauche et un micropoli mat et microgrenu, marqué de nombreuses stries perpendiculaires au fil. Ces usures se rattachent à un travail de raclage de bois. Les stries, révélant la présence de particules abrasives sur la matière d'œuvre, permettent d'envisager un travail d'écorçage.

Les chutes de burin ne présentent pas d'usure et ne semblent pas être le produit de ravivage de bords actifs. Aucun support brut analysé n'a donné lieu à une interprétation fonctionnelle. Les divers éléments des volumes de remontage examinés, en dehors de quelques stigmates postdépositionnels, sont vierges de trace d'utilisation, y compris les supports laminaires les plus réguliers, procédant de débitages plus investis réalisés aux dépens de silex roulé noir. Les petits éclats, mesurant entre $20 \mathrm{~mm}$ et $30 \mathrm{~mm}$, ne constituant pas les supports d'outils aménagés, ne semblent pas non plus servir en l'état, du moins dans ce locus. Il en est de même pour les petites lamelles de fin de chaîne qui ne sont pas transformées et qui ne paraissent pas avoir été utilisées brutes. La question de la destination de ces petits supports, éclats et lamelles, reste donc posée.

\section{Activités et fonctionnement du locus}

Hormis les activités cynégétiques dont témoignent indirectement les armatures, les processus techniques identifiés dans le locus 1 se rapportent essentiellement au travail de la peau et du bois pour la production de biens matériels. Le traitement des peaux est représenté par les grattoirs. Ces outils sont impliqués dans les opérations médianes à terminales de la chaîne opératoire, celle du drayage ou de l'assouplissement par raclage des peaux en cours de séchage ou reverdies. Les traces de découpe de peau sèche observées sur une pointe de Malaurie peuvent correspondre, quant à elles, à l'obtention de lanières ou de pièces de cuir par exemple. Le travail des végétaux ligneux, en particulier le travail du bois en cinématique transversale, est attesté sur plusieurs types d'outils: un burin, un éclat tronqué et une lame ébréchée. Ils ont servi pour différentes tâches, s'insérant dans une ou dans des chaînes opératoires distinctes. Ainsi, ont été réalisées des activités de raclage, vraisemblablement d'écorçage, de rabotage et de régularisation d'une pièce de bois pseudo-cylindrique, comme le fût d'une arme ou d'un instrument. Enfin, les macro et microtraces d'impact confirment le statut fonctionnel des armatures et, ainsi, montrent que la réfection des flèches et le remplacement des éléments des projectiles tirés ont été réalisés sur place. Ce locus pourrait donc constituer un pôle pour la fabrication et la réfection des armes et des instruments et plus généralement pour la production de biens matériels. L'hypothèse d'une segmentation spatiale des activités, qu'il convient d'envisager en corollaire de la fonction du locus, 


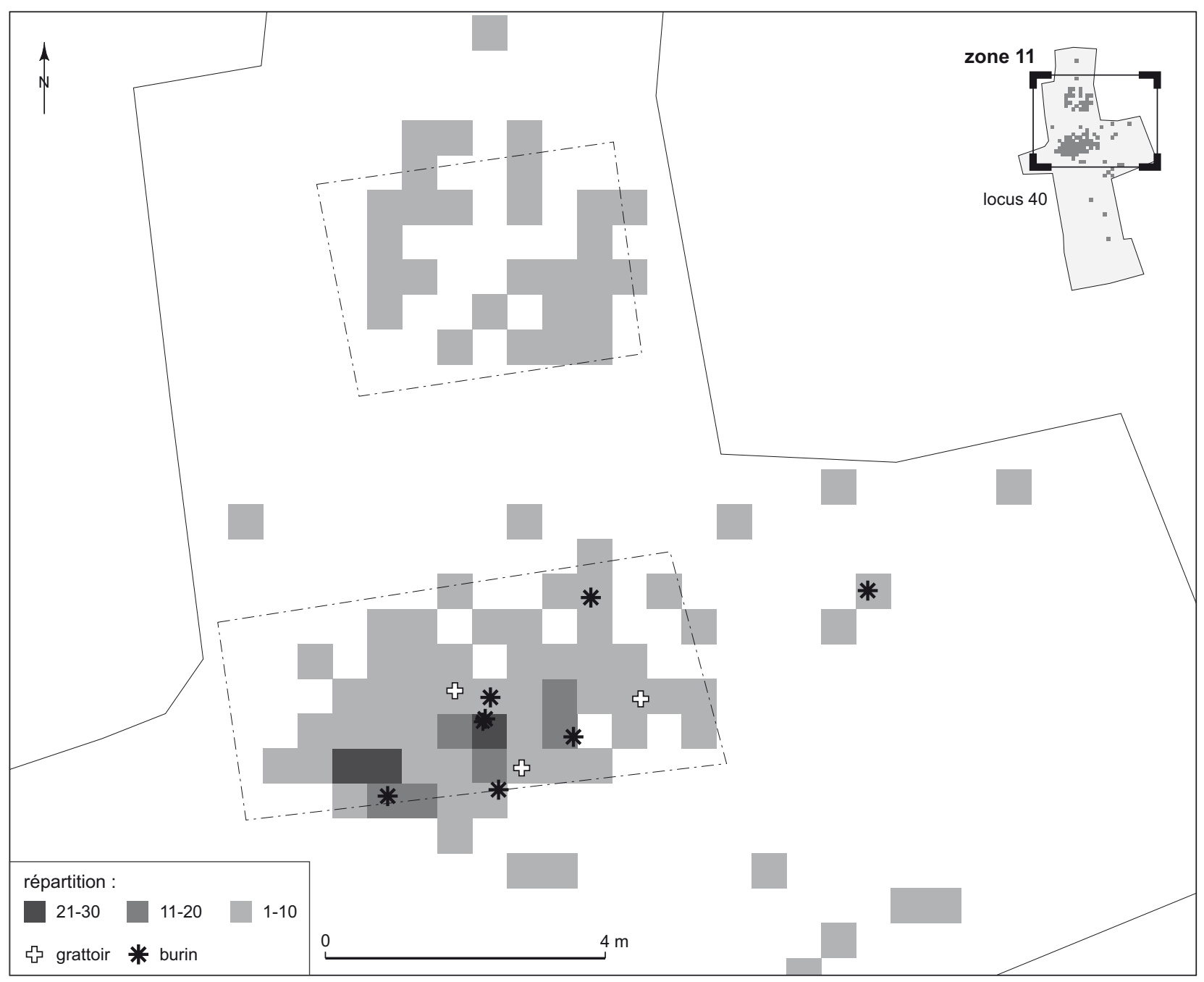

Fig. 55 - Les Chaloignes à Mozé-sur-Louet, locus 40, répartition des grattoirs et des burins. Les lignes tiretées signalent la zone totalement fouillée dans le locus. Représentation en quart de mètre carré (dessins: S. Sicard, Inrap et J.-F. Nauleau, Inrap).

semble étayée par les ruptures de certaines chaînes opératoires.

Ces ruptures concernent principalement les activités liées à l'exploitation des carcasses et des matières animales fraîches. Déjà suggérée par la sous-représentation des fragments apicaux d'armatures, l'absence des activités de boucherie montrerait que les processus d'acquisition de biens alimentaires, et en l'occurrence de la viande, ont été réalisés à l'extérieur du locus 1 . De même, aucune tâche impliquant les tissus osseux n'a été repérée. Au sein de la chaîne opératoire du traitement des peaux, se sont les premières étapes qui font défaut, l'écorchage et le décharnage notamment. Si ces ruptures sont bien réelles, et ne sont pas imputables à un biais taphonomique, elles plaident en faveur d'une complémentarité fonctionnelle inter-locus ou inter-sites qu'accréditent également l'exportation de supports et l'absence des lames à dos et de certains nucléus. Le locus 1 constituerait une aire relativement spécialisée au sein de l'espace économique et du système technique des Chaloignes.

\section{Conclusion}

Comme dans la plupart des contextes aziliens analysés en Europe, les modes de gestion des outillages du locus 1 des Chaloignes sont symptomatiques d'un faible investissement techno-fonctionnel. Ne constituant pas une réponse à un stress ou à une contrainte, bien au contraire, la sim- 
plification des techniques de débitage s'accompagne de comportements fonctionnels tout aussi libres; la façon dont sont employés les outillages en témoigne. Les différentes catégories de débitage identifiées dans le locus, qu'elles soient expédientes ou plus élaborées, et les diverses matières premières impliquées n'ont pas fait l'objet de cycles d'utilisation spécifiques. Une large part des produits lithiques obtenus est abandonnée sur place, sans avoir servi. Ce rapport production/utilisation pourrait être interprété en termes d'anticipation sur les besoins, mais peut-être faut-il envisager d'autres alternatives qui ne correspondent pas forcément à des choix rationnels. En tout état de cause, le statut non fonctionnel d'une partie de l'outillage n'est pas sans implication pour l'interprétation de la variabilité structurelle inter-locus.

S. P.

\section{LE LOGUS 40 : \\ UNE ABONDANCE DE BURINS}

Le décapage de la zone 11, située au sud de la cuvette et à proximité immédiate de la vaste zone 8, est intervenu assez tard dans le calendrier de l'opération et plusieurs orages estivaux ont rempli d'eau la totalité du sondage, retardant encore la fouille. Nous n'avons donc pas eu le temps de traiter toute la surface du sondage; en revanche les deux zones sélectionnées ont été fouillées exhaustivement (fig. 55). La collection comprend 350 pièces, posées directement sur le schiste sous $0,50 \mathrm{~m}$ de sédiment. Aucun élément intrus n'est perceptible au sein du matériel azilien, même si un petit fossé de parcellaire borde à l'ouest les concentrations. Cette zone avait été repérée une première fois lors de l'évaluation; 34 pièces doivent donc être ajoutées aux vestiges issus de la fouille, mais au moment de la phase d'évaluation aucune armature n'avait été trouvée pour permettre une attribution chronologique de ces silex. Ces derniers ne se surimposent pas directement sur la concentration, mais sont sis plus au sud de la zone 11. Même en l'absence de pointe azilienne, ces pièces sont cohérentes avec le reste du matériel, : une lame à bord abattu irrégulier, proche du couteau à dos le confirme. Il est fort probable que de nouveaux locus auraient été détectés alentours lors d'une fouille exhaustive.

\section{LA RÉPARTITION SPATIALE}

La fouille du locus 40 fut limitée à deux zones quadrangulaires; de ce fait, l'aire de répartition des vestiges est inférieure à celle des autres locus, le diamètre de la nappe de vestiges n'excédant pas 2,50 m. Les burins sont dispersés sur toute la surface, avec quelques grattoirs. Armatures aziliennes et nucléus s'excluent dans l'espace, comme dans le locus 1 où nous suggérions une même dynamique de dépôt: les armatures démanchées d'un côté et les nucléus de l'autre.

\section{LES MATIÈRES PREMIÈRES}

L'essentiel du silex utilisé sur ce locus provient de galets roulés (94,7\%, hors indéterminés). On peut mentionner la présence de quatre matières au cortex vif: D, G, J et L. Trois d'entre elles sont représentées par un unique éclat, tandis que la matière $\mathrm{G}$ apparaît sous la forme d'un fragment de nucléus. Ce sont donc des supports bruts qui n'ont pas été débités sur place; ils restent anecdotiques et n'ont pas de valeur pour la compréhension des chaînes opératoires de ce locus.

\section{LES OUTILS}

Avec $12,8 \%$, les armatures sont faiblement représentées au sein de l'outillage aménagé. Elles sont au nombre de cinq, toutes fragmentées. Il y a une pièce à bord abattu qui pourrait être une lamelle à bord abattu (fig. 56, n ${ }^{\circ} 7$ ), et quatre pointes aziliennes (fig. 56, $\mathrm{n}^{\text {os }} 1$ à 4). La latéralisation de ces dernières se situe indifféremment sur le bord droit ou gauche de la pièce. On peut mentionner, sur deux d'entre elles, une coche latérale, en partie proximale du tranchant. Les largeurs s'étalent entre $8 \mathrm{~mm}$ et $13 \mathrm{~mm}$, alors que les épaisseurs se situent toutes à $4 \mathrm{~mm}$. Elles sont réalisées sur des supports peu réguliers mais au profil rectiligne.

Dans l'outillage commun, les burins $(23,1 \%$ du total des outils communs) dominent nettement les grattoirs $(7,7 \%)$ et forment la catégorie la plus importante, devant les éclats retouchés. Ils occupent une place privilégiée dans ce locus; dans toutes les autres concentrations, ils dépassent rarement $10 \%$ des outils aménagés. Ici, ce sont principalement des burins sur troncature (les deux tiers de l'ensemble des burins), entiers et réalisés sur éclat allongé et large (fig. 56 et 57). Ils sont généralement d'angle, quelques fois plans. La partie active est le plus souvent large. Il existe aussi un burin double, dont les parties actives sont opposées et décalées. Les dimensions de ces burins n'indiquent pas de standardisation particulière. Plus les éclats sont longs, plus ils sont larges. En revanche, les épaisseurs ne semblent pas corrélées aux largeurs ou aux longueurs. Réalisés sur éclats 

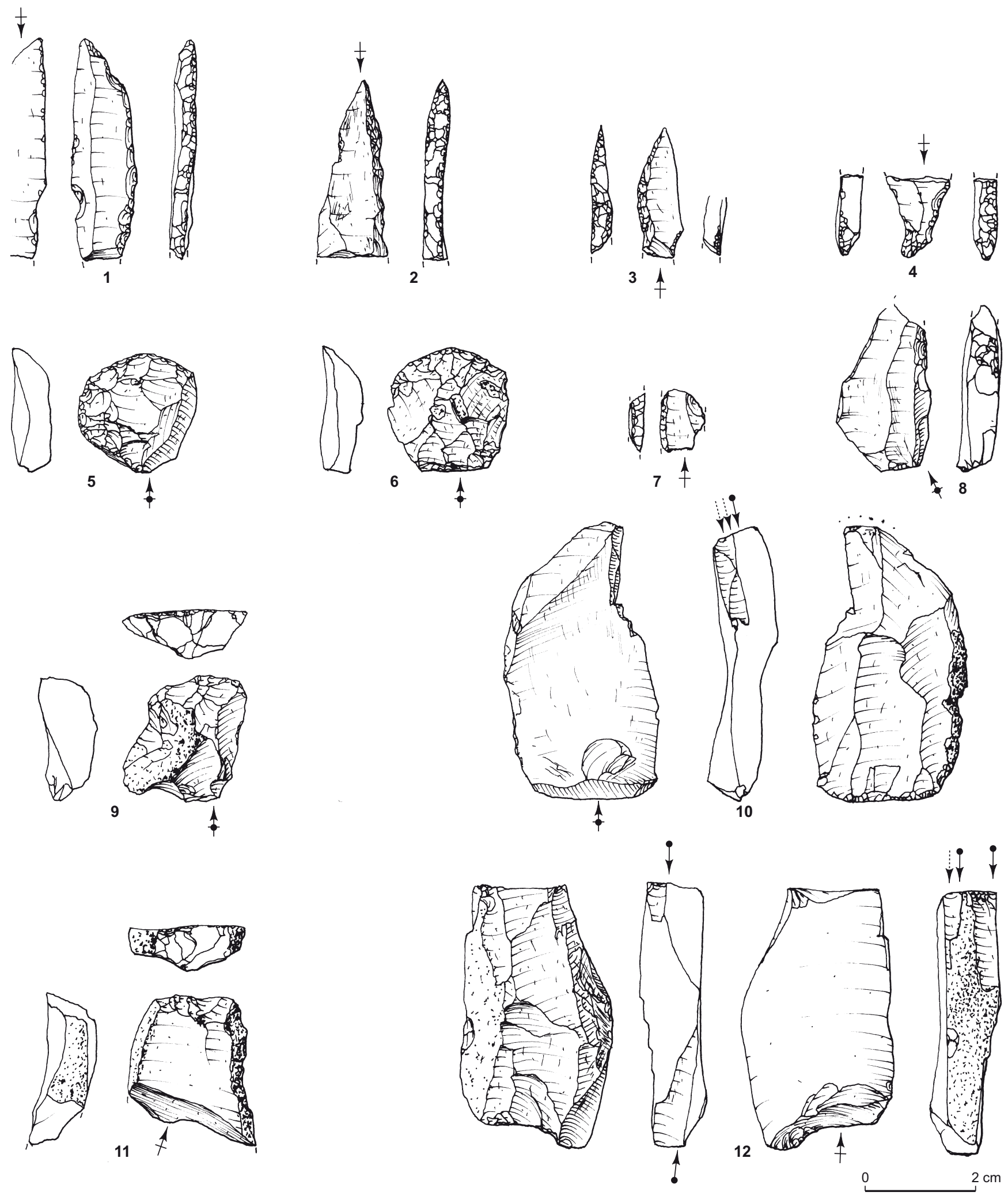

Fig. 56 - Les Chaloignes à Mozé-sur-Louet, locus 40. 1-4, pointes aziliennes entières ou fragmentées; 5 et 6, 9, 11, grattoirs; 7 , pièce à bord abattu; 8 , éclat ou lame à bord abattu; 10 et 12, burins (dessins: P. Forré, Inrap). 

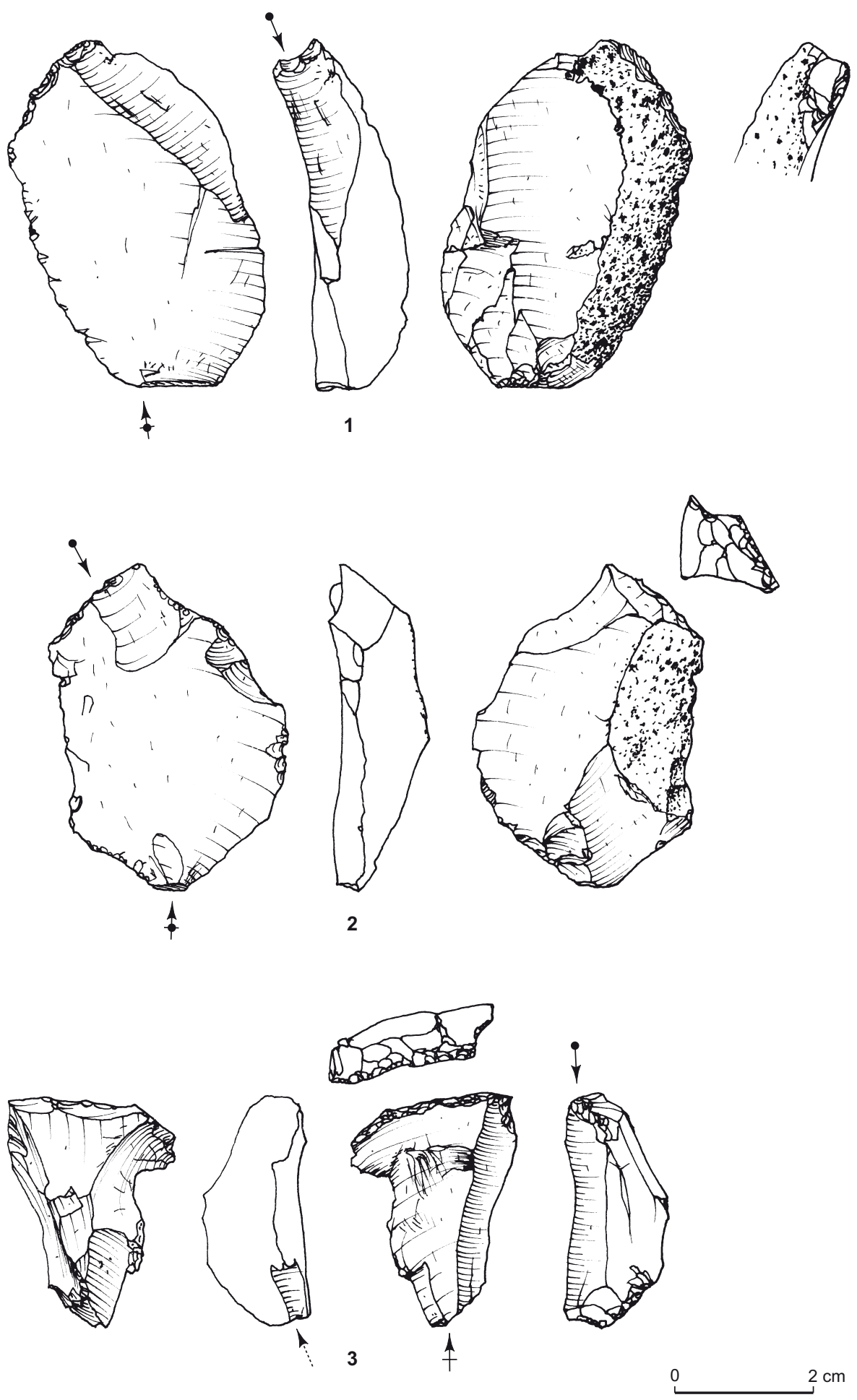

Fig. 57 - Les Chaloignes à Mozé-sur-Louet, locus 40. Burins sur troncature (dessins: P. Forré, Inrap). 
courts et larges, les grattoirs $(\mathrm{n}=3)$ n'ont pas de caractères particuliers. Leur front est arrondi, sans affecter la totalité des bords. Il existe enfin une faible proportion d'éclats retouchés et ébréchés. La retouche qui affecte ces produits est marginale, souvent partielle. À l'échelle macroscopique, il est parfois difficile de différencier les outils qui résultent d'un véritable aménagement de ceux qui sont liés à l'utilisation ou au piétinement. Signalons enfin la présence d'un éclat à bord abattu (fig. 56, nº 8), sans doute appointé, réalisé sur un support irrégulier.

\section{LE DÉBITAGE}

L'ensemble des lames et lamelles brutes est le plus restreint de tous les locus; il ne correspond qu'à 14,4\% du débitage. Cela pourrait se justifier par la faible représentation des armatures dans l'outillage et donc une orientation préférentielle des activités. Au contraire du locus précédent, on note une nette distinction entre les longueurs des lames de plein débitage et celles des sous-produits laminaires. Les premières sont très allongées - la moyenne est autour de $52 \mathrm{~mm}$ - alors que les secondes sont courtes et ne dépassent pas $40 \mathrm{~mm}$. Ces chiffres indiquent un débitage orienté vers la production de lames assez grandes, pour un usage indéterminé. L'étude des outils nous a permis de mettre en évidence le choix de supports allongés pour la fabrication des burins, au contraire des autres locus où ces outils sont réalisés sur des supports très variés mais généralement courts. Mais, dans le locus 40, les lames de plein débitage n'ont pas directement servi à la fabrication des burins; il n'en reste pas moins que cette orientation s'est reflétée dans les sous-produits laminaires, supports de ces outils.

On peut remarquer que le taux de produits corticaux et semi-corticaux est le même que sur les locus où les premières phases de la chaîne opératoire sont présentes. La taille résiduelle des nucléus laisse pourtant penser que le débitage n'a pas été exhaustif, les derniers enlèvements ayant une longueur toujours supérieure à $38 \mathrm{~mm}$. Ces nucléus représentent $3,4 \%$ des vestiges lithiques, ce qui correspond à peu près à la moyenne des locus homogènes. Il y a autant de nucléus à lames $(\mathrm{n}=4)$ que de nucléus à éclats $(n=5)$.

\section{FONCTIONNEMENT DE L'HABITAT AZILIEN DES CHALOIGNES}

\section{NATURE DES ACCUMULATIONS DE SILEX}

\section{Apport des remontages et du SIG}

L'utilisation du SIG (Système d'informations géographiques) Arcview, après un relevé du matériel en trois dimensions et la mise en place d'une base de données, a grandement facilité le croisement de toutes les informations dans l'espace, à l'échelle de chaque locus puis du site. Les interrogations générées sur ce logiciel ont permis de recouper des données difficilement manipulables sans le recours à l'informatique. La répartition des pièces selon leurs caractéristiques (type d'outils, nature du support, brûlés ou non, dimensions, matières premières ) a fait l'objet d'un examen systématique, par critère unique ou par association de critères pertinents.

Les blocs gélifs fragmentés sous forme de cassons ont été remontés presque entièrement sur chaque locus. Mais, pour le reste de la production, aucun volume n'a pu être reconstitué complètement, ce qui est un aspect fondamental des accumulations de ce site. Les premières phases de la chaîne opératoire sont fréquemment remontées, sans doute parce qu'elles sont les plus simples à reconnaître grâce aux plages corticales. Autre observation qui confirme les faibles remaniements post-dépositionnels: les derniers enlèvements sont souvent situés à proximité des nucléus. La phase de plein débitage laminaire est la plus difficile à remonter, à l'exception de celle du locus 1 , où de nombreuses séries ont été restituées. Cela est en partie dû à la grande fragmentation des pièces, et a fortiori des lames. Le statut de zone de rejet de certains locus peut aussi expliquer partiellement les manques, puisque les produits destinés à être transformés en outils n'ont pas été abandonnés avec le reste du débitage, mais prélevés et utilisés ailleurs.

Il n'y a que deux remontages inter-locus, entre les locus 1 et 2. Cela permet d'envisager des relations directes: les liens entre ces deux locus étaient déjà apparus nettement lors de l'étude lithique qui avait montré la similitude de leur équilibre typologique et leur complémentarité technique. C'est pourtant dans cette recherche des traces d'interaction que les tentatives ont été les plus nombreuses, puisque ces traces sont déterminantes pour juger de la contemporanéité entre locus. Ainsi, le silex naturellement rouge-sombre, arrivé sous forme de galets roulés et débités dans le locus 13, se rencontre à proximité dans le locus 12 et de manière plus étonnante dans le locus 3, à $150 \mathrm{~m}$ à l'est: mais comment 
démontrer un quelconque lien entre eux, qui plus est avec un bloc roulé? Pourtant, cette carence en remontages entre locus ne suffit pas strictement pour affirmer que les amas ne sont pas le résultat d'une seule occupation à l'échelle de la cuvette. S'ils ne sont pas déterminants pour cette dernière question, ils sont tout de même essentiels à la compréhension interne de chaque locus. Selon les amas, les remontages sont plutôt groupés ou dispersés. Ainsi, dans les locus 4, 11 ou encore 1, les pièces remontées peuvent être distantes de plusieurs mètres. Dans le locus 10 ou 13 , les pièces remontées sont beaucoup plus ramassées et indiquent une plus faible dispersion du matériel. Ces deux modes de répartition permettent de compléter notre vision de la dynamique spatiale de chaque locus, illustrant le mode d'accumulation des déchets rencontrés. Certes, l'absence de structuration n'est pas obligatoirement le reflet d'un rejet des produits de débitage hors de la zone de taille; ces derniers ont pu être dispersés et remaniés postérieurement à l'intérieur du locus. De ce fait, la position réciproque des éléments raccordés ne permettent pas de déterminer si le locus est en position primaire ou secondaire. Il faut un faisceau d'indices concordants pour proposer une telle interprétation; c'est ce que nous nous sommes attachés à trouver tout au long de l'étude lithique du matériel afin d'établir le statut et la fonction de chaque locus grâce à laquelle nous envisagerons de présenter une vision globale du site.

\section{Dispersion des matières premières}

À l'intérieur de chaque locus, la répartition des matières premières non roulées est souvent remarquable: on constate que dans les locus 10, 11 et la zone 5, les silex sont regroupés par matières. Sur les locus 10 et 11, les déchets de taille en matière $\mathrm{G}$ sont ramassés dans un petit espace du locus. Sur la zone 5 , les produits en matière exogène sont rassemblés sur deux petits espaces. Dans le locus 10, on retrouve cette même disposition, observée par le groupement des matières et des remontages. À l'inverse, sur le locus 4 où l'exploitation de la matière $\mathrm{M}$ a été très importante, les silex sont dispersés sur tout le locus, sans regroupement particulier. Ces deux configurations plaident aussi bien pour un débitage sur place que pour un rejet à l'aide d'une couverture ou d'un panier, avec une faible dispersion secondaire. La présence de matières premières non roulées différentes sur chaque locus est un élément important, qui laisserait imaginer des occupations successives. Toutefois, le fait que l'on retrouve quelques éléments de chacune de ces matières dans d'autres locus que celui où elles ont été débitées plaide au contraire pour l'existence d'un lien chronologique entre certains locus. Cela est valable pour la matière $\mathrm{M}$, pour le quartzite de Montbert et pour la matière G.

\section{Dispersion des armatures}

Les pointes à dos courbe sont souvent groupées, par exemple dans les locus 1, 4, 11 et 40, ainsi que dans la zone 5 . Dans le locus 11, les pointes sont toutes réunies sur une aire de $8 \mathrm{~m}^{2}$ (fig. 34), alors que la superficie totale du locus est de $35 \mathrm{~m}^{2}$; dans le locus 4 , les pointes sont rassemblées dans la moitié est. Dans la zone 5, on constate à l'inverse une forte densité d'armatures dans la partie ouest, alors qu'elles sont beaucoup moins nombreuses sur le reste de la zone. Dans les locus 1 et 40, ces armatures se regroupent grossièrement tout en s'excluant avec les nucléus. À l'échelle de chaque locus, ces armatures offrent donc une structuration qui serait le reflet d'activités distinctes, comme le remmanchement proposé dans le locus 1. Par ailleurs, des fractures de type Krukowski, qui signent des ratés de fabrication d'un dos, sont observées sur des lames étroites dans la zone 12 et dans les locus 1,10 et 13 . On en retrouve aussi, quoiqu'en moindre nombre, pour des pièces larges à bord abattu dans les locus 4 et 10, ainsi qu'en zone 5 .

\section{Dispersion de l'outillage commun}

Les répartitions des troncatures, des coches et les éclats aménagés à l'intérieur des locus ont fait l'objet d'une observation systématique, mais aucune structuration particulière n'est apparue. Cela est un résultat en soi, qui distingue ces outils des armatures, des grattoirs et des burins pour lesquels on a pu noter quelques indices de structuration. Les denticulés sont toujours très rares, ne dépassant pas $3 \%$, sauf dans le locus 2 où ils représentent $5 \%$ des outils aménagés. Les troncatures sont plus abondantes, entre $4 \%$ et $14 \%$ selon les locus; dans le locus 13 , elles forment presque $20 \%$ du total des outils aménagés. Au contraire, elles sont totalement absentes du locus 3 . Il est fort probable que ces outils n'ont pas fait l'objet d'un seul mode d'utilisation: ils peuvent donc matérialiser des activités différentes. Enfin, les éclats aménagés sont également répartis sur tous les locus, formant une catégorie numériquement importante mais hétérogène, dont il serait vain de chercher la cohésion spatiale. On peut seulement souligner le déficit de cet ensemble typologique dans le locus 1, qui ne représente que $19 \%$ des ensembles des outils retouchés contre 
plus de $20 \%$ et jusqu'à $40 \%$ partout ailleurs. Cela renforce encore l'idée d'un locus dédié à une activité particulière. Il aurait pu être profitable de considérer la répartition conjointe de deux types, éventuellement engagés dans une même activité. Cependant, le fonctionnement de ces outils communs n'est pas connu.

\section{Dispersion des produits de débitage}

À l'intérieur de chaque locus, l'observation de la répartition spatiale de tous les éléments du débitage offre peu de résultats. Les nucléus, alors qu'ils se répartissent plutôt en périphérie du locus 1 ou au centre du locus 13, sont en général dispersés sur toute la surface des autres accumulations. Les lames, qu'elles soient de plein débitage ou non, se retrouvent partout, proportionnellement à la densité totale de matériel. Les différentes phases de la chaîne opératoire tels que la mise en forme ou le plein débitage laminaire n'apparaissent jamais groupées sur de petits espaces. Enfin, à l'exception du locus 12, l'observation de la répartition des talons facettés n'a pas donné de résultats qui auraient pu matérialiser l'usage de ce procédé à un moment donné du débitage ou le savoir-faire particulier d'un tailleur.

\section{L'organisation intra-locus}

Dans la zone 5 et dans les locus 1 et 10, des éléments du Mésolithique moyen ou récent sont apparus. Il y a donc eu une pollution partielle de ces amas, qui a peut-être conduit à une déstructuration de l'espace azilien. La zone 4 - siège d'un bâtiment gaulois - montre une absence d'organisation claire, qui ne reflète en rien la répartition originelle des vestiges. Comme le reste de la cuvette, ce versant ensoleillé et bien drainé a fait l'objet d'occupations intenses, laissant des concentrations de matériels lithiques. La conservation différentielle des vestiges nous a conduit à centrer nos études comparatives sur les locus intégralement préservés des bords de la cuvette.

L'étude précise de la répartition spatiale interne des locus a révélé des organisations significatives, par exemple l'opposition armature/nucléus des locus 1 et 40 ou les regroupements d'armatures. La dispersion aléatoire des outils et des éléments du débitage observée dans certains locus est souvent inexploitable pour la lecture spatiale; elle corrobore l'hypothèse d'amas correspondant à des zones d'activités multiples, ou plus rarement orientées, et certainement pas à des amas de débitage en position primaire. Ces derniers, fouillés par exemple sur un site comme le
Closeau (Rueil-Malmaison, Hauts-de-Seine; Bodu, 2000a et 2000b), livrent des regroupements particuliers de nucléus ou de lames, un très faible taux d'outils, une dispersion du matériel de chaque amas sur des surfaces de moins de $1 \mathrm{~m}^{2}$ et des remontages très nombreux. Toutes ces caractéristiques ne se retrouvent pas aux Chaloignes, même dans les locus 4 et 13. La dispersion du matériel est nette sur des surfaces pouvant atteindre $70 \mathrm{~m}^{2}$, le pourcentage d'outils n'est pas négligeable et les remontages peuvent affecter des pièces très éloignées les unes des autres. Les locus des Chaloignes sont tout de même, dans certains cas, des aires d'accumulation primaire: à l'issue des activités, les outils ou les déchets sont abandonnés sur place. Le locus 1 comporte un grand nombre d'armatures, des lames étroites cassées lors de la confection d'un dos (fracture Krukowski) et une réserve importante de lames de plein débitage, en rapport direct avec la fabrication ou la réfection d'armes. Le locus 13 et, avec plus d'incertitudes, le locus 10 pourraient correspondre à de véritables zones de débitage sur place si l'on considère la cohérence spatiale des remontages, la sousreprésentation de la phase laminaire de plein débitage, le groupement des matières premières non roulées (pour le locus 13), le faible nombre d'outils et, pour le locus 10, la poche de «percuteurs». Les autres locus peuvent aussi être des aires de rejets, à proximité des habitations ou des aires d'activités primaires, voire des accumulations mixtes, à la fois aires de rejet et aires de travail. Il convient maintenant de chercher les orientations techniques et fonctionnelles de chaque locus en confrontant plusieurs catégories de faits (tabl. XV).

Tabl. XV - Recherche d'associations de paramètres. Les proportions d'armatures, de burins et de grattoirs sont exprimées en pourcentages relatifs au sein des outils.

\begin{tabular}{|l|c|c|c|c|c|}
\cline { 2 - 6 } \multicolumn{1}{c|}{} & $\begin{array}{c}\text { Débitage } \\
\text { laminaire } \\
(\mathrm{x}>25 \%)\end{array}$ & $\begin{array}{c}\text { Brûlé } \\
(\mathrm{x}>40 \%)\end{array}$ & $\begin{array}{c}\text { Armature } \\
(\mathrm{x}>40 \%)\end{array}$ & $\begin{array}{c}\text { Burin } \\
(\mathrm{x}>20 \%)\end{array}$ & $\begin{array}{c}\text { Grattoir } \\
(\mathrm{x}>20 \%)\end{array}$ \\
\hline Locus 1 & & $\mathrm{X}$ & $\mathrm{X}$ & & \\
\hline Locus 2 & & $\mathrm{X}$ & $\mathrm{X}$ & & \\
\hline Locus 3 & & & $\mathrm{X}$ & $\mathrm{X}$ & \\
\hline Locus 40 & & $\mathrm{X}$ & & $\mathrm{X}$ & \\
\hline Locus 13 & $\mathrm{X}$ & & & $\mathrm{X}$ & \\
\hline Locus 11 & & $\mathrm{X}$ & & & $\mathrm{X}$ \\
\hline Locus 12 & & & & & $\mathrm{X}$ \\
\hline Locus 4 & $\mathrm{X}$ & & & & $\mathrm{X}$ \\
\hline Zone 13 & & & & & $\mathrm{X}$ \\
\hline Locus 10 & & & & & \\
\hline
\end{tabular}




\section{TYPOLOGIE DES LOCUS}

\section{Caractères généraux}

Avant même de comparer les assemblages de chaque locus, le plan général du site nous permet de constater la disposition régulière de ces concentrations, espacées de $10 \mathrm{~m}$ à $15 \mathrm{~m}$ (fig. 14 et 59). Leur diamètre mesure de $5 \mathrm{~m}$ à $7 \mathrm{~m}$. Chacune est bien délimitée dans l'espace alors que tant de sites mésolithiques offrent l'image de vastes nappes de vestiges. Les deux secteurs qui présentent un type d'organisation légèrement différent, plus étalés, sont le locus 10 et la zone 5 ; ils étaient d'ailleurs probablement liés, mais la route a empêché d'en juger lors de l'intervention. Sur la zone 5, aucun véritable locus n'a été mis au jour, mais le plan de répartition du matériel montre quatre concentrations qui peuvent correspondre à des aires aux contours gommés par la superposition de plusieurs occupations préhistoriques.

Lorsque l'on considère la carte de densité en nombre de témoins lithiques, on note de forts contrastes entre les locus. On peut établir une hiérarchie entre les petits locus qui contiennent moins de 500 pièces et ceux qui en présentent le double, les locus 11, 10 et 1 étant, par ordre décroissant, les plus importants. Les petits locus sont majoritaires, mais ne sont pas regroupés dans un espace particulier du site. Au contraire, il est fréquent de trouver en association une grosse et une petite accumulation, sans y voir pourtant aucune complémentarité. Tous les locus ont la même morphologie ovale, avec une aire plus dense de silex au centre. Leurs superficies, comprises entre $10 \mathrm{~m}^{2}$ et $70 \mathrm{~m}^{2}$, sont corrélées à leur importance numérique. Il faut bien admettre que des zones très peu denses ont pu disparaître lors du décapage à la pelle mécanique, tant il fut difficile de caler l'action de la pelle mécanique sur un niveau précis.

Malgré l'absence de structures foyères, des liens entre ces aires d'activités ou de rejets et le feu peuvent être appréciés. Les chiffres liés au pourcentage de silex brûlé au sein de chaque locus permettent de les répartir en deux ensembles distincts: il y a des concentrations à faibles pourcentages de matériel altéré par le feu (moins de $25 \%$ de matériel brûlé), et des concentrations dont plus de la moitié des silex est brûlée (fig. 58). Les locus 1 et 2 sont marqués par le feu et se distinguent ainsi de la zone 3 qui est peu brûlée. Dans la zone 8, le matériel est peu brûlé et seul le locus 11 s'individualise par un fort pourcentage. Les zones 4, 5 et 11 font parties de l'ensemble bien touché par le feu. Cette répartition hétérogène dans la cuvette ne plaide pas en faveur de l'hypothèse d'un incendie général, postérieur à l'occupation azilienne. Sur le locus 11, il n'est

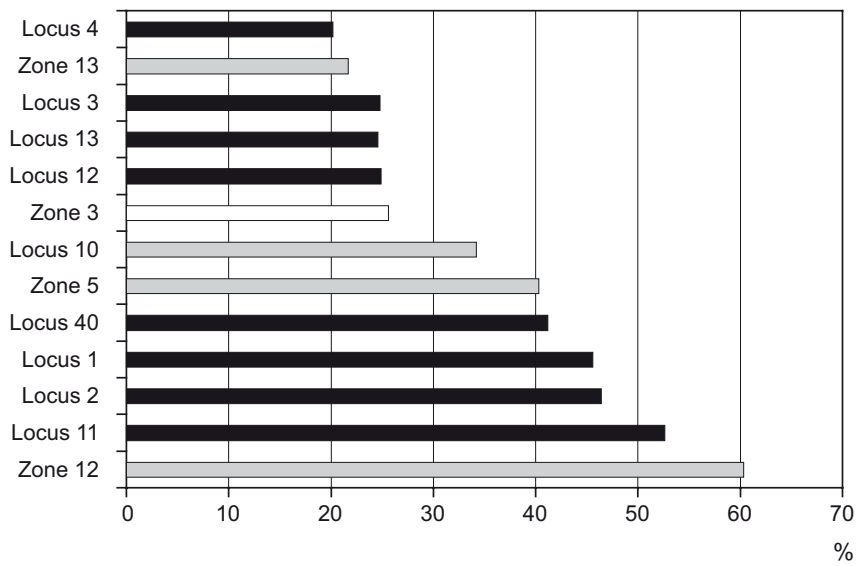

Fig. 58 - Proportions de matériel lithique brûlé par zone ou par locus. En noir, les concentrations aziliennes homogènes, en hachuré, les concentrations à intrusions postérieures minimes, en blanc, les concentrations très perturbées.

pas rare de raccorder une pièce brûlée et une pièce intacte, alors qu'elles sont situées à proximité l'une de l'autre. Ce caractère plaide alors pour une action du feu qui se placerait entre le débitage et l'abandon définitif de la pièce. Si l'on ne peut établir de corrélation directe entre le pourcentage de pièces brûlées et le type d'outils dominant dans chaque locus (tabl. VII), on peut cependant remarquer que les locus considérés comme des zones de premier débitage, comme le locus 13 ou le locus 4, sont beaucoup moins affectés par le feu que ceux qui semblent correspondre à des zones d'activités plus «spécifiques» comme le locus 40 ou encore les locus 1 et 2. Il est possible alors que la chauffe des pièces, loin d'être aléatoire, soit la résultante d'une utilisation du feu, non pas lors du débitage, mais lors d'activités domestiques distinctes.

L'ensemble des locus présente un taux de fractionnement comparable, très élevé avec plus de $65 \%$ du matériel cassé. Nous avons constaté que ce taux est plus faible sur d'autres sites contemporains comme Hangest-sur-Somme ou Conty (Somme) qui présentent les mêmes conditions taphonomiques que les Chaloignes (Fagnard, 1997). Il est possible, étant donné les faibles dépôts sédimentaires sur le site angevin, que le matériel resté à l'air libre ou protégé par très peu de sédiment se soit plus rapidement dégradé.

Le silex roulé a été utilisé majoritairement dans tous les locus. Il représente plus de $80 \%$ des silex. Il faut noter la seule exception que constitue le locus 4 avec seulement $39,1 \%$ de matières roulées. Le silex majoritaire y est non roulé, beige veiné brun, fin avec un cortex feuilleté pulvérulent (matière M). Parmi les autres locus, seul le locus 10 
a connu l'utilisation d'une matière première non roulée de manière importante (silex G). On peut souligner la diversité de ces silex non roulés et l'utilisation privilégiée de l'une d'entre elles dans chaque locus. À chaque fois, le volume de matière première non roulée débitée dans un locus ne représente guère plus d'un bloc.

Les pointes aziliennes et les pièces à bord abattu sont présentes sur tous les locus. L'étude précise de ces pièces a montré une grande homogénéité dans leur typologie et donc un faible pouvoir discriminant dans la recherche d'une éventuelle diachronie. Les deux seules bipointes aux Chaloignes se situent sur la zone 5, et leur signification chronologique n'a pas été déterminée en l'absence de tout autre élément allant dans le sens d'une occupation azilienne ancienne. Il y a donc une unité culturelle indéniable entre toutes les concentrations. Les monopointes aziliennes sont une trentaine sur le site, réparties entre tous les locus. Dans le locus 1, les armatures représentent le type d'outils majoritaire; c'est d'ailleurs le seul du site à offrir un tel corpus de pointes. Cette observation peut-être mise en relation avec l'existence d'une chaîne opératoire laminaire de plein débitage qui confère à nos yeux un statut particulier à ce locus. Plusieurs armatures de diverses périodes ont été décrites précédemment. Leur présence au milieu de quelques locus comme le 10 ou la zone 5 marque sans doute l'existence de petites occupations plus récentes, au Mésolithique moyen ou au Néolithique récent. Cependant, les vestiges laissés par chacune d'entre elles sont trop ténus pour déterminer le lieu précis d'habitat. Cela ne nous paraît pas avoir affecté de manière importante la répartition des vestiges aziliens, ni remettre en cause l'attribution chronologique à l'Azilien de tous les locus.

Il existe une corrélation inverse entre les proportions de burins et de grattoirs (tabl. XV). Lorsque le taux de grattoirs est élevé, celui des burins est faible et l'inverse est aussi vrai. Il existe même des exclusions comme sur le locus 3 où il y $25 \%$ de burins alors qu'il n'y a aucun grattoir et le locus 2 où il n'y a pas de burin mais $9,5 \%$ de grattoirs. Ces derniers ne sont donc majoritaires que sur cinq des neuf locus. Lorsque l'on examine leur répartition à l'échelle du site, cela n'offre aucun résultat interprétable (fig. 59): les «locus à burins» ne sont pas groupés dans un secteur particulier. Par locus, on constate aussi des zones où les grattoirs et les burins ne sont pas mélangés, comme sur le locus 10 . Cela reste pourtant anecdotique: en effet, sur tous les autres locus les deux types d'outils couvrent la même surface. L'observation du rapport grattoir/burin n'est toutefois pas anodine et plusieurs hypothèses peuvent être émises quant à sa signification. Il est fréquent d'accorder une valeur chronologique au résultat de ce rapport. Ainsi, au Bois Ragot (Gouex, Vienne; Chollet, Dujardin, 2005) ou à Pont d'Ambon (Bourdeilles, Dordogne; Célérier, 1994 et 1998), dans l'Azilien ancien, les burins sont plus nombreux que les grattoirs puis cela s'inverse dans l'Azilien récent. Le fait que les deux types de rapport coexistent aux Chaloignes pourrait donc indiquer l'existence de plusieurs phases d'occupation sur le site. Cependant, aucun autre élément typologique ou technique ne vient corroborer cette idée et, au contraire, nous avons pu démontrer l'extrême homogénéité des données lithiques de l'ensemble des locus. Il nous paraît donc plus vraisemblable que ces pourcentages d'outils soient le reflet d'activités distinctes générant plus d'outils spécifiques à un moment, même si cela reste généralement peu marqué dans les chiffres et peu lisible dans l'espace. Il n'y a que dans le locus 40 que le pourcentage de burins se distingue vraiment de celui des autres locus; il concentre en effet à lui seul près de $25 \%$ des burins du site, dont une majorité de burins sur troncature; ces derniers représentent $17,6 \%$ des outils contre $5,9 \%$ pour les burins sur cassure, alors que partout ailleurs ces derniers sont majoritaires. Ce seul chiffre ne nous permet pourtant pas d'affirmer de manière définitive le statut fonctionnel particulier de ce locus, qui par ailleurs présente les mêmes caractéristiques techniques que les autres locus.

\section{Déclinaisons mineures du débitage}

D'un locus à l'autre, les données techniques sont comparables, l'ensemble utilisant le schème opératoire azilien. Il est difficile de trouver des particularités permettant de conclure à des comportements techniques propres à un locus. Par exemple, un taux élevé d'éclats corticaux n'est absolument pas représentatif d'un faible niveau d'avancement de la chaîne opératoire: le NMI est de 12,2\% dans le locus 1 contre $5,5 \%$ dans le locus 13 ou 3,8\% dans le locus 4 , alors que le premier se distingue des deux autres par un plus grand avancement du débitage et par des témoignages de fabrication d'armatures. On peut prétendre que les petits éclats corticaux, qui interviennent à n'importe quel moment des chaînes opératoires, brouillent ce paramètre. Hors, si l'on écarte les pièces de classe dimensionnelle 1 , le taux d'éclats corticaux ou semi-corticaux reste beaucoup plus faible dans les locus 4 et 13 (dits de premier débitage) que dans le locus 1 . Il en va de même si l'on ne traite que les pièces supérieures à $30 \mathrm{~mm}$, soit une autre manière de définir les gros éclats corticaux. La segmentation des 


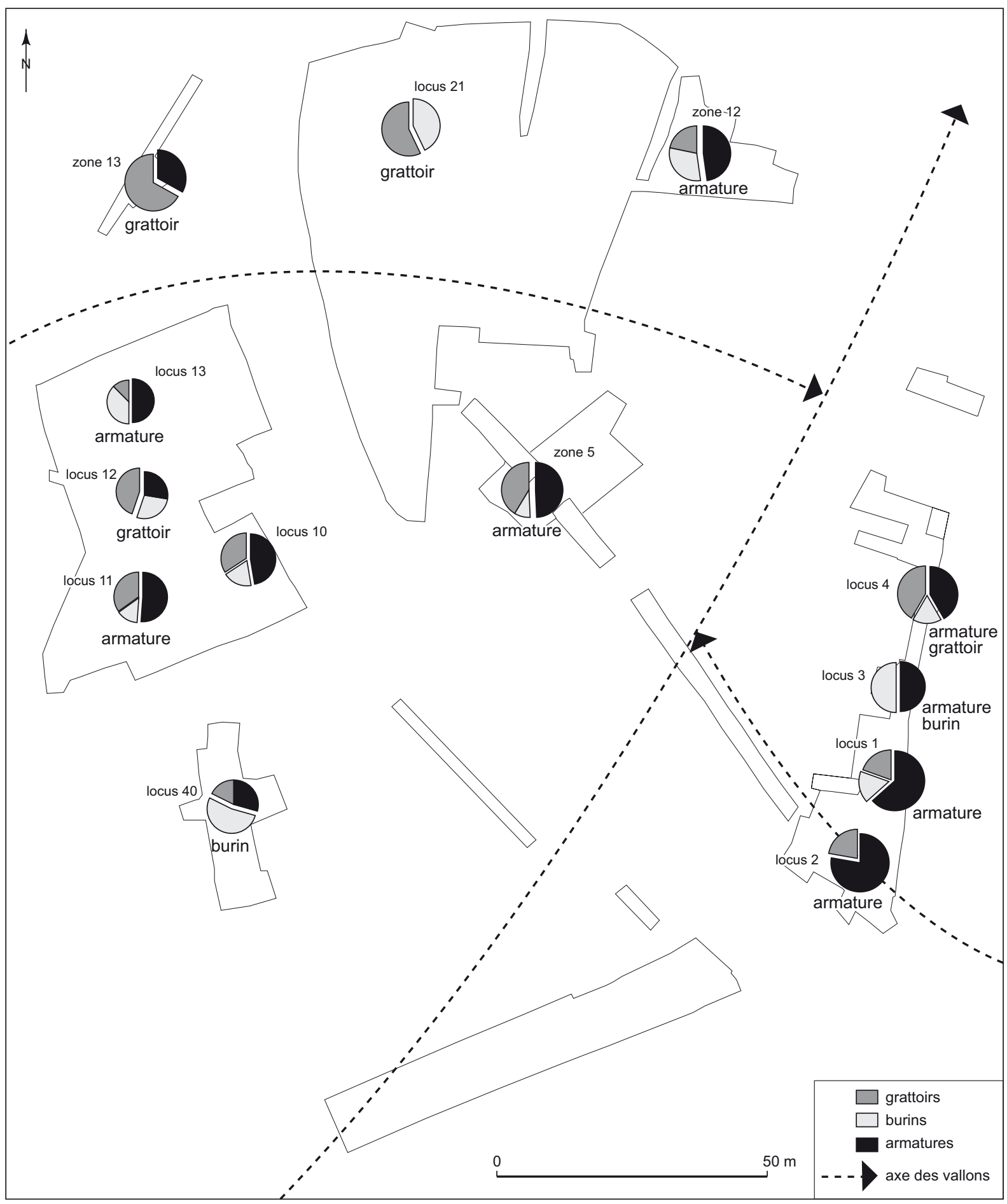

Fig. 59 - Proportions des armatures grattoirs, burins et armatures dans les zones aziliennes homogènes et non-homogènes. La catégorie dominante est mentionnée à côté du locus (dessins: S. Sicard, Inrap). 
chaînes opératoires est donc difficilement quantifiable dans ce système technique aux normes peu rigides.

Le débitage varie au gré des activités, sans cependant que l'on puisse établir de distinctions strictes entre les locus :

- le locus 10 montre un déficit de produits laminaires de plein débitage,

- le locus 13 présente un corpus d'éléments bruts particuliers qui semble correspondre à un véritable amas de débitage où les premières phases de la chaîne opératoire sont surreprésentées,

- le locus 3 correspond à un débitage de fin de chaîne opératoire,

- le locus 1 présente principalement un débitage laminaire de plein débitage et une phase d'aménagement d'armatures,

- le locus 11 est une accumulation «moyenne », où l'ensemble du débitage est abandonné sur place.

Il existe donc des locus qui se rapprochent d'aires de débitage (locus 4, 10 et 13) et d'autres qui évoquent des espaces de rejet (locus 11 et 2). Il est enfin possible que certains locus correspondent à des zones d'activités spécifiques (locus 40 et 1), mais les indices sont assez ténus dans ces locus, qui comprennent tout de même toute la panoplie azilienne.

\section{L'organisation inter-locus}

Plusieurs caractères propres à chaque locus sont apparus, qui permettent de proposer pour certains un «statut» particulier: fabrication et réparation des armatures en locus 1, premier débitage laminaire pour les locus 4 et 13, travail de l'os ou du bois pour les burins du locus 40. Une analyse factorielle des correspondances a été réalisée en collaboration avec A. Tresset (CNRS - Muséum d'histoire naturelle) afin de détecter d'autres paramètres discriminants entre les locus. Les 44 variables prises en compte comprennent les principaux produits de débitage bruts et les outils. Les facteurs 1 et 2 représentent 47,41\% des données (fig. 60). La quantité de débitage laminaire (lame de plein débitage ou autre, lamelle de plein débitage), et dans une moindre mesure celle des cassons et des burins sur troncature, apparaissent comme déterminantes pour séparer le corpus en deux: les locus 1,4 et 13 se différenciant des autres. Les outils, presque uniformément répartis, ne sont pas des éléments discriminants entre locus. Cette bipartition ne correspond pas à celle proposée à partir d'autres caractères dans les pages précédentes, mais elle isole quand même trois locus pour lesquels nous avons proposé une orientation des activités. Cette absence de discrimination stricte renvoie encore une fois à une image générale d'activités multiples sur les locus, parfois orientées, mais jamais spécialisées. À ce stade de l'analyse, un nouveau bond dans l'interprétation est nécessaire pour discerner le fonctionnement de ces habitats aziliens; il implique de maîtriser la chronologie relative des occupations, à partir de quelques indices seulement.

\section{DES OCCUPATIONS SIMULTANÉES OU FRACTIONNÉES?}

La répartition des différents locus en zones 1, 3, 8, 11 et 12 suggère que nous sommes en présence d'un niveau d'occupation organisé, où les amas n'ont subi que quelques altérations mineures; ces dernières n'ont sans doute déformé que très partiellement le dépôt, le décapage à la pelle mécanique étant finalement la phase la plus destructrice. Avant d'émettre une ou plusieurs hypothèses de fonctionnement du site, il convient de s'interroger sur la pertinence d'une étude globale et comparative de ces locus en termes spatiaux. Les limites spatiales imposées par l'emprise autoroutière et par le temps de fouille ne nous donnent qu'une vision partielle de l'espace habité. Notre réflexion repose donc sur des données incomplètes. L'étude technique et typologique seule nous a donc permis d'établir le fait que tous les locus correspondent à un même horizon chronologique. Au-delà, la répartition des vestiges en locus témoignerait soit d'une occupation unique, soit de plusieurs occupations dont la durée et les éventuelles successions saisonnières n'ont pu être mises en évidence. En l'absence de tout vestige autre que le silex, nous n'avons pas accès à des éléments de compréhension complémentaires de ceux apportés par l'étude du système technique azilien. Ainsi, l'aspect saisonnier de l'occupation, sa durée et même sa fonction pourraient être envisagés grâce aux restes de faune ou encore aux charbons L'éventualité d'une occupation unique, de durée indéterminable, peut être défendue par plusieurs arguments :

- tout le matériel est compris dans un même horizon sédimentaire, même si la faible sédimentation générale peut expliquer cet unique horizon;

- le matériel est peu dispersé verticalement, chaque locus étant épais de moins de $20 \mathrm{~cm}$;

- l'organisation évidente de l'espace, avec des zones vides, des locus régulièrement espacés et des zones plus larges à très fortes densités pourrait indiquer une mise en place synchrone de tous ces locus; 
Facteur $2(18,58 \%)$

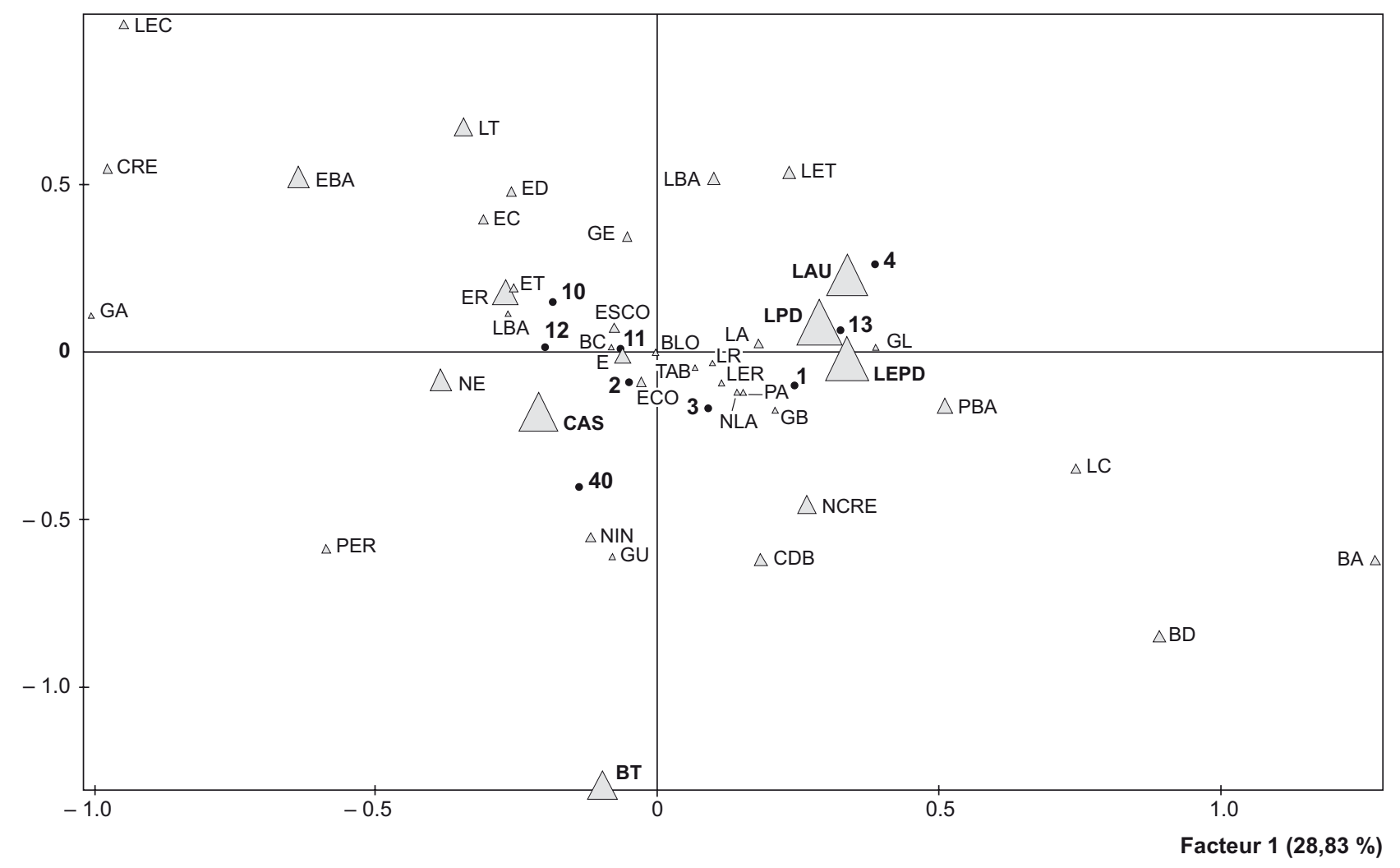

\begin{tabular}{|c|c|c|c|}
\hline ECO. éclat cortical & NLA. nucléus à lame & AD. armature diverse & LET. lamelle à troncature \\
\hline ESCO. éclat semi-cortical & NE. nucléus à éclat & GU. grattoir unguiforme & EBA. éclat à bord abattu \\
\hline E. éclat & NIN. nucléus indéterminé & GL. grattoir / lame & LBA. lame à bord abattu \\
\hline lame de plein débitage & CAS. casson & GA. grattoir autre & PER. perçoir \\
\hline LAU. lame autre & BLO. bloc & GB. grattoir + burin & ED. éclat denticulé \\
\hline LEPD. lamelle de plein débitage & PA. pointe azilienne & BD. burin dièdre & EC. éclat à coche \\
\hline LA. lamelle autre & LBA. lame étroite à bord abattu & BT. burin / troncature & LC. lame à coche \\
\hline CDB. chute de burin & PBA. pièce à bord abattu & BC. burin / cassure & LEC. lamelle à coche \\
\hline CRE. crête & SE. segment & BA. burin autre & ER. éclat retouché \\
\hline NCRE. néo-crête & SC. triangle scalène ou isocèle & ET. éclat à troncature & LR. lame retouchée \\
\hline TAB. tablette & TR. trapèze & LT. lame à troncature & LER. lamelle retouchée \\
\hline
\end{tabular}

Fig. 60 - Plan factoriel 1-2 de l'analyse en AFC de 9 locus pour 44 variables. Les 2 axes représentent 47,41 \% des données (réalisé en collaboration avec A. Tresset, CNRS UMR 5197).

- la mise au jour ponctuelle de quelques éclats ou lames en matières non roulées dans un locus, alors que l'exploitation de cette matière est attestée dans un autre locus, pourrait illustrer des contacts directs.

Les arguments en faveur d'occupations successives sont aussi nombreux:

- à cause du faible enregistrement sédimentaire, les vestiges laissés sur le sol d'habitat devaient être encore visibles lors des réoccupations successives et conditionner le choix des lieux du campement, ce qui peut expliquer les évitements; - certaines zones très denses, comme la zone 5 , pourraient être le reflet de réoccupations successives de cet endroit qui a progressivement conduit à une superposition de plusieurs locus ;

- l'absence de complémentarité avérée entre les locus et l'absence de locus véritablement spécialisé pourraient plaider pour des occupations déconnectées dans le temps.

À l'issue de la présentation des arguments sur la nature de l'occupation, il semble évident qu'aucun d'entre eux n'est assez déterminant pour arriver à une conclusion définitive. Il est possible que certains locus aient fonctionné simultanément, comme cela semble être le cas pour les locus 1 et 2. 
De manière beaucoup plus conjecturale, la présence d'un même silex roulé rouge opaque dans les locus 12,13 et 3 pourrait laisser croire en des échanges. Ces observations obligent à rester prudent quant à l'organisation et au fonctionnement du site, le raisonnement fondé sur la représentation différentielle des activités à partir de la lecture des chaînes opératoires nécessitant d'admettre un lien entre tous les locus. À l'inverse, il faut rappeler l'absence de structures en pierres: peut-on utiliser cet argument pour qualifier le mode d'occupation aux Chaloignes? Il s'agirait alors d'une succession de haltes de courte durée.

\section{CHRONOLOGIE VERSUS FONCTION}

La variabilité des assemblages lithiques a été longuement étudiée dans les lignes précédentes. Elle s'exprime dans un cadre typologique assez strict qui confère à l'ensemble une évidente monotonie. La carence des datations par le radiocarbone et par la stratigraphie reste un handicap pour le site des Chaloignes, surtout lorsque l'on veut explorer les causes de ces variations souvent mineures. Le recours préférentiel à des matières autochtones, le débitage bipolaire sur une même table, le facettage des plans de frappe, le profil rectiligne des lames, l'usage d'un percuteur de pierre, les monopointes à dos courbe, les grattoirs circulaires et les burins peu standardisés sont autant de caractères qui ne laissent guère de doute quant à une attribution de la plupart des locus des Chaloignes à l'Azilien. La tripartition chronologique de ces groupes à pointes à dos courbe semble être une réalité sur tout le territoire français avec, pour les armatures, une succession bipointes / monopointes / pointes de Malaurie (Thévenin, 1997; Bodu, 2000a). Les locus des Chaloignes offrent suffisamment d'arguments pour s'intégrer tous dans une phase récente de l'Azilien, soit la phase à monopointes :

- la mise en forme est simplifiée, les entames corticales sont nombreuses,

- le débitage laminaire, réalisé par percussion directe dure, est peu régulier, l'essentiel de la calibration des supports revenant à l'aménagement par la retouche,

- les bipointes sont rares, tandis que les pointes de Malaurie sont presque absentes,

- les grattoirs courts ou unguiformes sur éclats dominent largement en nombre sur les grattoirs sur lames,

- la morphologie des burins est hétéroclite.

$\mathrm{Au}$ sein de cet horizon chronologique, que représentent les variations entre locus? Dans la magistrale monographie du site de Rekem, fouillé dans le Limbourg belge, J.-P. Caspar et M. De Bie observent un fort taux de réfection pour les burins, avec en conséquence des changements de types; ils considèrent alors que «les observations faites également sur la dynamique de ces outils remettent en question l'utilisation abusive des catégories typologiques comme paramètres diagnostiques d'éventuels faciès régionaux dans les groupes à Federmesser» (De Bie, Caspar, 2000). De manière plus ou moins inconsciente, nous avons aussi opté pour des interprétations fonctionnelles, en raison de la monotonie dans la gamme des armatures ou dans les caractères techniques. Il est probable que d'autres chercheurs verront dans la diversité des locus des modifications d'origine diachronique, comme le veut une tradition classique de la recherche sur le Paléolithique et le Mésolithique en France. Si la catégorie très homogène des armatures offre ici peu de prise pour cette approche, le rapport grattoir/burin pourrait être considéré comme caractéristique de l'évolution typologique à la fin du Paléolithique, les premiers augmentant progressivement au cours du temps, jusqu'à la disparition totale des burins au Mésolithique moyen. Aux Chaloignes, la succession des locus se ferait donc comme suit, du plus «ancien» au plus «récent»: 3 (sans grattoir), 13 et 40, 1, 12, 10, 11 et 2 (sans burin). La modicité des échantillons rend l'exercice assez inapte à rendre compte de la chronologie interne du gisement. L'intérêt des Chaloignes est autre, d'une part dans un essai de compréhension paléo-ethnographique de l'habitat, d'autre part dans la définition d'entités techniques aziliennes encore uniques dans l'Ouest.

Cette unicité confère une valeur exemplaire au site des Chaloignes, qui incite à tirer des enseignements pour les travaux ultérieurs dans des contextes sédimentaires semblables. Force est de constater qu'un locus fouillé isolément aurait à chaque fois fourni des éléments suffisamment diagnostiques pour permettre une attribution à l'Azilien. Cela implique d'une part que la spécialisation des zones d'activités n'est jamais suffisante pour brouiller la diagnose chrono-culturelle, d'autre part qu'il y a une faible segmentation des activités dans l'espace du site. Une trop grande prise en compte des fossiles-directeurs dans le diagnostic fonctionnel est peut-être partiellement responsable de cet état de fait, qui entraînerait une sous-évaluation des spécialisations de chaque locus. À défaut d'analyse tracéologique totale, nous éviterons des lectures fonctionnelles par trop univoques. Quoi qu'il en soit, si l'on se place au final dans la perspective des futures recherches sur l'Azilien dans l'ouest de la France, il faudra se souvenir que, quelles que soient les orientations supposées des activités, la quasi-totalité 
de l'outillage azilien est abandonnée sur chaque locus. En d'autres termes, l'attribution chrono-culturelle et la compréhension des principaux processus techniques sont possibles pour cette période à partir d'ensembles restreints.

\section{ÉLÉMENTS DE RÉFLEXION SUR L'ANALYSE SPATIALE À PARTIR D'AUTRES SITES STRUCTURÉS DE PLEIN AIR}

Les comparaisons avec d'autres sites préhistoriques de plein air n'ont pas la prétention d'émettre un modèle général d'organisation et de fonctionnement. Au contraire, il nous semble que l'analyse spatiale intra-site ne doit pas acquérir de valeur générale, la perception de l'organisation pouvant varier considérablement selon la fonction du site, sa durée d'occupation et la nature des vestiges rencontrés sur le sol d'habitat.

Aux Chaloignes, les produits de taille sont regroupés et forment de gros amas bien circonscrits. Les vestiges sont donc organisés dans un espace ouvert, même si a priori la distribution des vestiges ne reflète pas la pratique d'activités très segmentées dans l'espace. Sur certains sites magdaléniens de plein air, tels que Étiolles (Essonne; Pigeot, 1987; Coudret et al., 1994) ou Pincevent (La Grande-Paroisse, Seine-et-Marne; Leroi-Gourhan, Brézillon, 1972; David, Orliac, 1994; Bodu et al., 2006), l'existence d'un espace interne, matérialisé par un abri, organisé autour d'un foyer et d'un espace extérieur, permet de caractériser ainsi un espace domestique et une zone de rejet. Toutefois, l'étude lithique du site d'Étiolles, couplée avec les remontages, a permis de distinguer dans cet espace extérieur des locus correspondant à des zones de rejet ou «amas poubelle» et de véritables ateliers de taille. Comme aux Chaloignes, ce sont la présence d'une organisation spatiale des produits de débitage en fonction de la chaîne opératoire, l'existence de remontages de séquences de débitage et enfin le regroupement de certaines matières premières qui ont permis de définir le statut des amas de débitage. Les deux types de locus ont donc bien pu coexister dans un espace restreint, même en dehors de l'espace considéré comme domestique. L'absence de foyer et d'abri ne signifie pas que l'on soit en présence d'un espace de rejet, dénué d'organisation.

Sensiblement contemporain, le site de Rekem (Limbourg belge) présente une répartition spatiale des vestiges proche de celle des Chaloignes (De Bie, Caspar, 2000). Il est interprété comme un vaste campement résidentiel. Les seize concentrations d'inégales importances comprennent des zones domestiques de $50 \mathrm{~m}^{2}$ à $60 \mathrm{~m}^{2}$ autour de foyers, des postes spécialisés de débitage et de façonnage d'outils et une seule aire de rejet. Elles s'organisent suivant deux axes parallèles, opposant les zones domestiques et les aires spécialisées. Les nombreux remontages indiquent des rapports entre locus éloignés. Au Closeau (RueilMalmaison, Hauts-de-Seine), trois niveaux d'occupation des groupes à Federmesser ont été fouillés sur une terrasse de la Seine sous la direction de P. Bodu (Bodu, 1995, 2000a et 2000b). Le niveau azilien ancien, daté autour de 12000 BP (soit l'intervalle 12500-11800 avant notre ère), montre un aménagement de l'espace par de grandes dalles de pierre pesant jusqu'à $50 \mathrm{~kg}$, avec des locus centrés autour d'un foyer (Bodu, 2000b). Cette organisation se démarque nettement de celle du niveau supérieur, dont les locus sont essentiellement matérialisés par des nappes de matériel archéologique, avec de rares foyers plus ou moins excentrés. C'est justement ce niveau qui offre des similitudes techniques avec les Chaloignes. Les sites à Federmesser de la vallée de la Somme correspondraient plutôt à des occupations uniques limitées dans le temps, avec une dispersion réduite des vestiges, centrés autour d'un unique foyer (Coudret, Fagnart, 1997). Mais il convient de remarquer que plus la surface fouillée est réduite, plus le campement devient une station logistique sous la plume de l'archéologue.

Qu'advient-il au cours du premier Mésolithique? Les éléments de réponse sont rares, car les habitats fouillés sur de vastes étendues sont peu nombreux. Sur le site mésolithique ancien de Choisey «Aux Champins» (Jura), des petits postes de débitage sont distincts de deux unités d'habitation aux activités multiples qui s'organisent autour de foyers. Il n'y a pas d'aménagements lourds, mais des effets de parois et des espaces vides semblant désigner des espaces particuliers (zone de couchage?). À la «Daupharde» (Ruffeysur-Seille, Jura), les vestiges paraissent s'organiser par locus, sans que «les produits de débitage ne définissent de secteurs d'activités spécifiques » (Séara, 1998 et 2000; Séara et al., 2002). Toutefois, les pièces lithiques se concentrent également autour des foyers, majoritairement à plat sans bordure ni cuvette. Cette organisation spatiale, qui se matérialise souvent par de larges nappes de vestiges, semble être fréquente au Mésolithique, sans que l'on puisse encore discriminer ce qui relève d'un faible enregistrement sédimentaire en climat tempéré, d'occupations courtes répétées ou encore de l'absence de segmentation spatiale des activités.

Seule structure d'habitat évidente, en général, sur les sites de cette période, alors que les trous de piquets ou les murets font souvent défaut, le foyer a eu au moins le mérite de réchauffer l'imaginaire des préhistoriens, pour lesquels 
l'âtre central est le principe primordial de la vie préhistorique. Aux Chaloignes, on ne distingue aucun espace vide qui pourrait correspondre à un négatif de foyer non empierré se rapprochant de ceux de Ruffey-sur-Seille; il n'y a pas non plus de «taches» de pièces brûlées bien circonscrites au sein des épandages lithiques. Enfin, aucun effet de paroi n'a été détecté. Notre lecture des locus a montré à la fois des activités variées sur chaque unité spatiale et une spécialisation très partielle de certaines zones. La notion de complémentarité spatiale est donc très mal illustrée sur ce site. Si les hommes n'établissent plus de structures lourdes, est-ce parce qu'ils se déplacent plus fréquemment? Est-ce parce que le climat moins rude n'impose plus de se protéger aussi fortement? Ces déterminismes sont fréquemment battus en brèche par des contre-exemples ethnographiques. Cette faible structuration de l'espace apparaît comme un caractère récurrent au cours de l'Azilien récent; elle trouve des prolongements au Mésolithique. Il faut remarquer que cette mutation est plus ou moins synchrone d'un approvisionnement plus local en matières premières et d'une moindre exigence dans la production des supports à retoucher; mais comment interpréter cette métamorphose? Ce sont des orientations qui ne seront remises en question qu'au Néolithique ancien, malgré quelques changements au Mésolithique récent. L'organisation des habitats participe à l'évidence de l'empreinte culturelle du groupe, avec un rythme évolutif plus lent que l'outillage. Les rapports sociaux et la nature des activités vont entraîner aussi une dispersion particulière des vestiges, mais les preuves archéologiques n'amènent jamais à des conclusions sans appel. L'existence de zones spécialisées et de débitages complexes au Magdalénien n'a jamais été interprétée comme l'acte de naissance de l'artisanat ou comme une preuve de hiérarchie sociale, alors que ces conclusions apparaîtraient immédiatement pour lire des configurations similaires au Néolithique. De même, les larges nappes de vestiges lithiques du Mésolithique moyen français sont censées témoigner du passage répété de petits groupes, sans que les auteurs ne prennent jamais la peine de démontrer pourquoi ils refusent l'idée de vastes villages sédentaires! Par la nature même de ses témoignages archéologiques dont la lecture se prête mal à une quelconque codification, l'habitat préhistorique semble être un miroir des fantasmes contemporains, un des derniers espaces de liberté dans lequel l'archéologue peut exprimer sa propension au rêve. En ce sens, notre proposition de lecture de l'habitat des Chaloignes témoigne d'une fantaisie limitée. Souhaitons seulement que ce texte présente de manière suffisamment claire les données pour permettre à l'imagination du lecteur de s'exprimer.

Lorsque l'on tente d'appliquer ces informations spatiales brouillées sur un plus vaste espace - le territoire économique dans lequel il s'insère - l'information perd encore de sa pertinence. Dans l'immédiat, rien ne nous indique l'existence de «stations logistiques» opposée à des «campements résidentiels» dans l'Azilien de l'Ouest, parce que nous sommes bien en peine de trouver une segmentation économique des chaînes opératoires au sein même des campements. D'ailleurs, la cuvette des Chaloignes pourrait être un «grand site» composé de «stations logistiques». Certes, l'analyse fonctionnelle des locus a montré des ruptures systématiques des chaînes opératoires de production et de consommation; mais il est difficile de savoir si le relais est pris ailleurs dans l'habitat ou autre part dans le territoire. Tout le débat réside dans un contrôle de la chronologie relative, dont nous avons suffisamment vu qu'il est impossible sur ce site.

Pour conclure, nous pouvons rappeler que les occupations des Chaloignes se font dans un court intervalle de temps selon la typo-chronologie, avec une importance certaine des activités de taille au vu de la quantité de vestiges déposés, avec des individus qui se détachent du groupe pour la réalisation d'activités assez diversifiées. Cette notion de séparation physique des individus est finalement comprise dans la détermination d'un locus, à savoir un espace domestique pour reprendre la terminologie de F. Séara (Séara et al., 2002). Il reste difficile à partir des observations archéologiques réalisées en ces pages, de proposer un modèle de fonctionnement catégorique pour rendre compte de ce fractionnement spatial observé. Dans le cas d'un vaste campement résidentiel, une communauté composée de plusieurs familles réside ici sur une longue période et, au gré des activités, des individus s'isolent en périphérie de la cuvette pour tailler du silex, réparer des flèches, travailler l'os, etc. Dans le cas d'une succession de haltes en un même lieu (une succession de sites logistiques), une famille, deux familles, une poignée de chasseurs, viennent périodiquement aux Chaloignes pour des séjours de courtes durées, afin de pratiquer des activités diversifiées, peut-être liées au grand fleuve qui court $2 \mathrm{~km}$ au nord. Ses rivages sont peut-être trop inconfortables et la cuvette des Chaloignes offre un abri relatif contre les vents. Dans cette interprétation, nous avons évité de faire référence à des réunions périodiques de plusieurs communautés dans cette cuvette abritée, pour des «potlatchs» annuels, ou pour d'autres occupations de l'espace propre à 
des chasseurs-collecteurs. Quelle que soit l'image mentale que le lecteur souhaitera conserver, il faut bien convenir que la cuvette des Chaloignes est probablement un nœud important dans le territoire azilien et, pour le Préhistorien, l'unique fenêtre encore ouverte sur les comportements techniques des hommes de cette période.

G. M. et S. S.

\section{LES GALETS GRAVÉS: UNE EXPRESSION SYMBOLIQUE DU PALÉOLITHIQUE TERMINAL}

\section{TROIS GALETS GRAVÉS SUR LE LOCUS 11}

Deux galets couverts de fines gravures parallèles ont été trouvés dans le locus 11 et un autre gisait à $2 \mathrm{~m}$ au nord de cette accumulation (fig. 61 à 63). Il s'agit de galets roulés de schiste ardoisier, très allongés, dont la surface est hélas parfois desquamée. Ils ont également en commun une extrémité naturelle en biseau et une section grossièrement quadrangulaire comprenant deux grandes faces. Les gravures sont des traits minces longitudinaux ou transversaux, réalisés sans reprise, très probablement à l'aide d'un tranchant de silex. Nous proposons une graduation suivant la profondeur de l'empreinte: incisions, fines gravures et très fines gravures. En prenant en compte les travaux expérimentaux de F. D'Errico concernant les principes de lectures des traits et notamment de leurs intersections (D'Errico, 1994), il est assez aisé de reconstituer la succession des séquences de gravure à l'aide d'une loupe binoculaire (grossissement maximum de 75 fois). En revanche, l'ordre d'exécution à l'intérieur des séquences et le sens d'exécution n'a pas été décrit, faute d'indice probant.

Le $n^{\circ} 1148$ est un galet de schiste, à feuilles parallèles (fig. 61 et 63), d'aspect légèrement satiné. Il est cassé à l'une de ses extrémités et ne mesure plus que $99 \mathrm{~mm}$. Sa largeur et son épaisseur sont également altérées par des cassures qui suivent les plans de clivage de la roche, mais les courbures conservées laissent cependant penser que la largeur de $21 \mathrm{~mm}$ est proche de l'originale. L'épaisseur de $11 \mathrm{~mm}$ n'est en revanche pas représentative. L'unique extrémité originelle restante affecte une forme en biseau, qui ne doit rien à un aménagement ou à une abrasion, mais correspond à un clivage naturel de la roche. Les surfaces lisibles sont, d'une part un bord allongé, d'autre part quelques parcelles d'une face très desquamée. Sur le bord, on observe treize incisions parallèles, distantes de $2 \mathrm{~mm}$ à $3,5 \mathrm{~mm}$, orthogonales au sens d'allongement du galet. Certes plus marquées que les autres gravures des galets des Chaloignes, ces stries n'ont été réalisées qu'en une seule fois, avec parfois des traces parasites, toutes dirigées vers une même face, ce qui implique un mouvement de même direction. Même si les faces sont très altérées, on peut assurer que ces traits ne reliaient pas tout à fait les deux faces plates. Une coche peu profonde a affecté ce bord après la réalisation des gravures. Si l'on en croit les traces, elle serait due à une percussion assez violente sur un objet minéral. Deux autres parties de ce bord sont transformées par des coches moins profondes et légèrement usées, postérieures aux incisions: leur usage est évidemment énigmatique. Les traits que l'on observe sur la face large encore partiellement lisible ne couvrent que $100 \mathrm{~mm}^{2}$. Ils forment un treillis de lignes très fines. Un essai de «stratigraphie» laisse voir trois actions successives: une première action de grattage qui se matérialise par des traces d'usure parallèles presque invisibles à l'œil nu, puis des traits longitudinaux, et enfin des traits transversaux.

Le galet $\mathrm{n}^{\circ} 120$ (fig. 63) présente des surfaces non desquamées qui révèlent un grand nombre de gravures, mais il a été cassé en longueur et en largeur, après l'exécution des traits. Ses dimensions actuelles sont de $113 \mathrm{~mm}$, pour une largeur et une épaisseur de $24 \mathrm{~mm}$. La surface A porte des traits longitudinaux très fins, réalisés au moins en deux séquences: l'une avec un changement d'axe en cours de réalisation (au moins quatre traits coudés), l'autre avec un certain parallélisme (au moins quatre traits rectilignes). Il est difficile de percevoir la chronologie d'un trait solitaire et des deux séquences évidemment sécantes. Leur succèdent neuf gravures fines transversales plus marquées, parallèles, avec un espacement régulier de $3 \mathrm{~mm}$ à $4 \mathrm{~mm}$; deux groupes de deux traits sont regroupés et convergents. La surface B est l'un des bords minces du galet. Les fines gravures parallèles, au nombre de sept, sont affectées de courts tracés parasites, comme pour la face $\mathrm{C}$, mais à la différence de la face A: c'est un indice fort pour un changement d'outil en cours de réalisation (mais avec quel laps de temps entre les deux?). Même si les gravures fines transversales vont jusqu'aux bords de ce galet assez anguleux, elles ne sont que très rarement en continuité. La face $\mathrm{C}$ est principalement affectée par les traits transversaux, incisés avec la même vigueur que sur les faces A et B. Au nombre de 19, ils sont grossièrement parallèles, à l'exception de 3 d'entre eux qui sont coudés. Ces inflexions peuvent s'expliquer par un accident de la surface, qui a fait dévier deux traits, les deux suivants semblent alors avoir récupéré le parallélisme: si cette interprétation est la bonne, la succession aurait été réalisée de la droite vers la gauche du galet (suivant le 


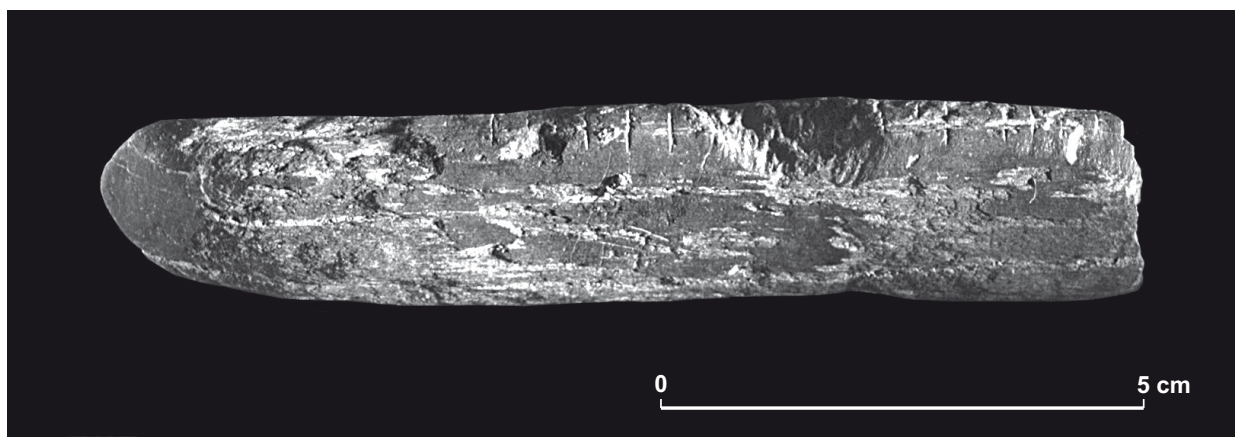

Fig. 61 - Les Chaloignes à Mozé-surLouet, locus 11. Galet gravé n 1148 (cliché H. Paitier, Inrap).

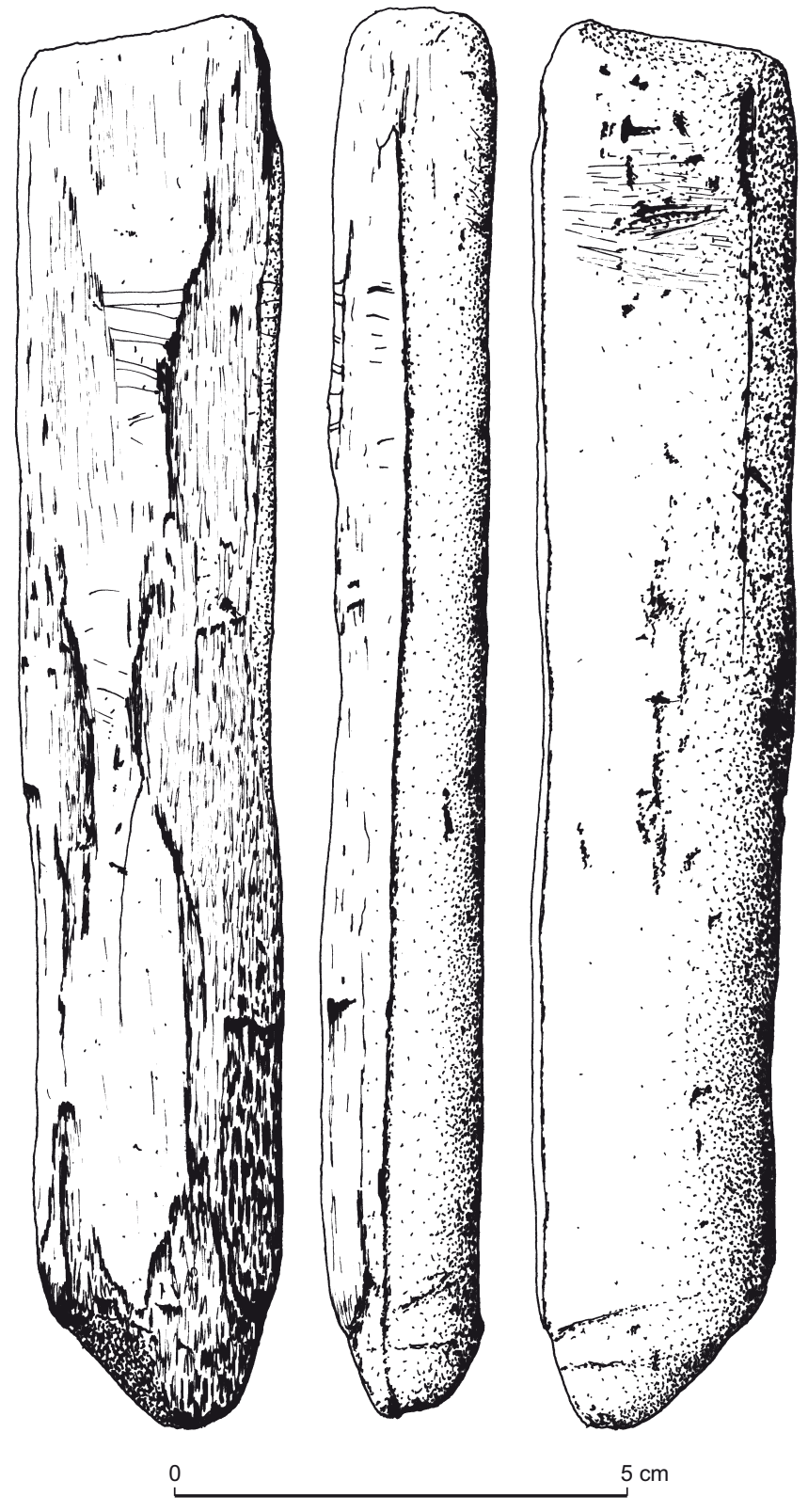

Fig. 62 - Les Chaloignes à Mozé-surLouet, locus 11. Galet gravé $n^{\circ} 87$ (dessins: P. Forré, Inrap). 


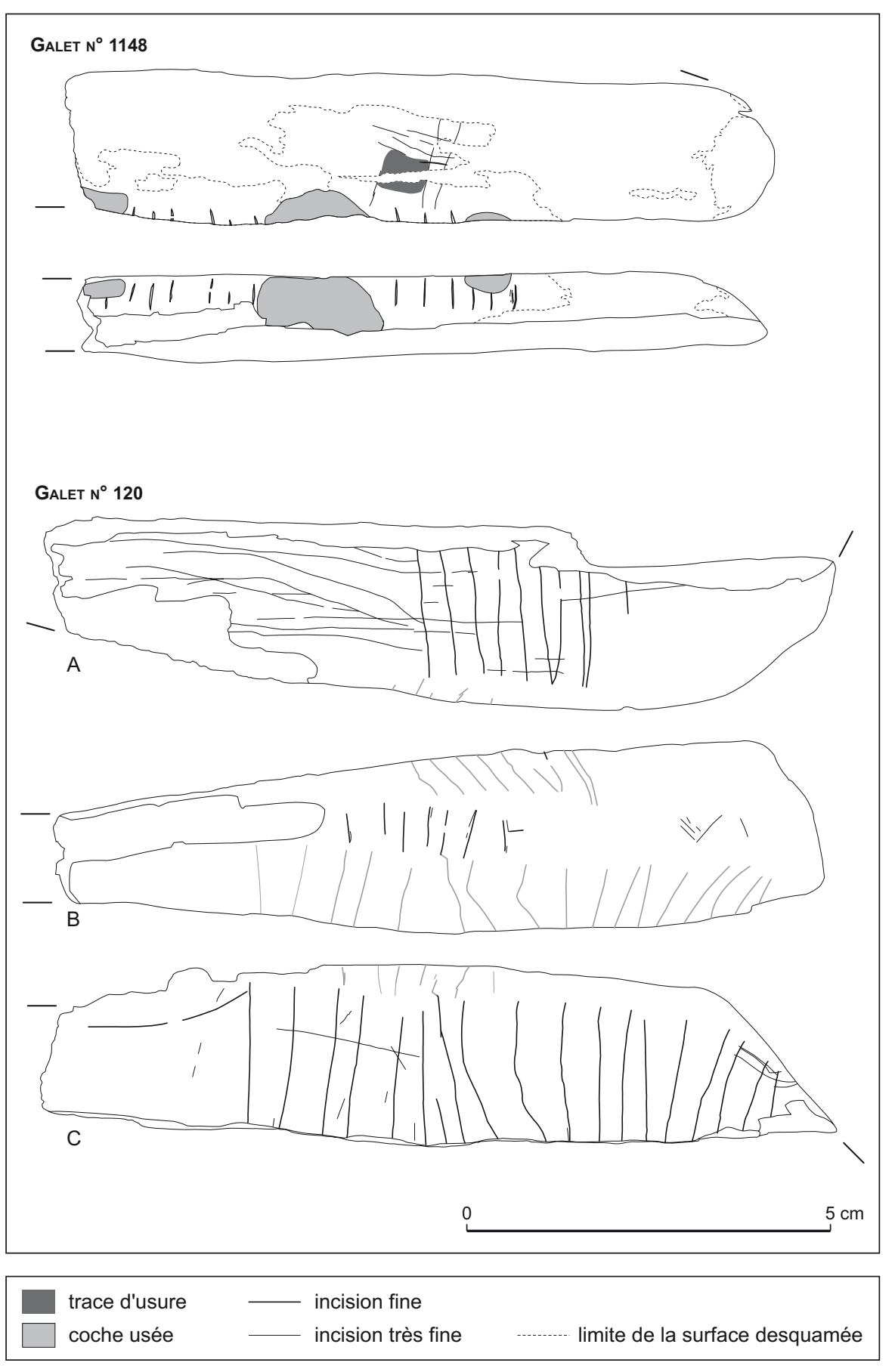

Fig. 63 - Les Chaloignes à Mozé-sur-Louet, locus 11. Relevé des galets $n^{\circ} 120$ et 1148 (analyse et dessins: G. Marchand, CNRS UMR 6566).

$\begin{array}{ll}\text { trace d'usure } & \quad \text { incision fine } \\ \text { coche usée } & \quad \text { incision très fine } \quad \text {-...-...- limite de la surface desquamée }\end{array}$

dessin de la fig. 63). Quelques traits longitudinaux très fins, probablement antérieurs aux traits transversaux, affectent également cette surface.

Le $n^{\circ} 87$ est un galet entier de schiste (fig. 62), notablement desquamé sur l'une de ses faces. Il mesure $155 \mathrm{~mm}$ x $27 \mathrm{~mm}$ x $16 \mathrm{~mm}$. Sa section est plus arrondie que celles des deux autres galets. Deux petites veines de quartz sont présentes à l'une des extrémités; elles sont orthogonales aux plans de clivage. Les gravures sont de très fines stries subparallèles discontinues, observables principalement sur la face desquamée et un bord. Ces traits forment trois groupes de deux ou trois lignes, mais il n'est pas possible 
de restituer un rythme à cause de l'altération. Des traces de percussion apparaissent au milieu de l'autre face, ainsi qu'une zone de fines rayures parallèles: l'ensemble évoque plutôt des traces d'utilisation comme enclume.

L'interprétation des gravures du galet $n^{\circ} 87$ est la moins assurée, seul le parallélisme des traits sur une face nous incite à quitter un champ strictement fonctionnel pour supposer d'éventuelles marques à valeur de signes. Les autres galets montrent plusieurs séries d'interventions: si les traits longitudinaux peu marqués peuvent éventuellement être d'origine fonctionnelle, les traits transversaux régulièrement espacés entraînent inéluctablement l'analyse vers une interprétation symbolique.

\section{QUESTIONS SANS RÉPONSE AUTOUR D'UNE EXPRESSION SYMBOLIQUE}

La thématique simple des décors, la matière, les dimensions et la forme des galets confèrent à l'ensemble des Chaloignes une grande unité de style. Les galets couverts de traits transversaux parallèles, réalisés sans mouvements de va-et-vient, sont des éléments récurrents sur les sites aziliens. F. D'Errico (D'Errico, 1994) a pu contester les hypothèses de système de notation (Marshack, 1972) et de calendrier (Couraud, 1985) en démontrant que les traits avaient été gravés rapidement, très souvent avec un même outil. Les deux séries de traits parallèles opposés, séparés par un espace vide, sont une des représentations les plus courantes (type Rochedane, Villars-sous-Dampjoux, Doubs ; Thévenin, 1983), qu'il interprète - après d'autres - comme une schématisation de l'être humain, avec les réserves d'usage. Aussi courantes dans les corpus, les séries de traits parallèles ne trouvent pas d'interprétation univoque. La fragmentation des supports aux Chaloignes empêche de déterminer si les séries parallèles sont uniques ou doubles, ces dernières étant inconnues d'ordinaire en basse et moyenne vallée de la Loire. On rappellera simplement ici, à partir des galets du locus 11, que la succession des traces se fait par séquences et non pas dans le plus grand désordre. La correction du trait déformé dans une inégalité du galet $n^{\circ} 120$ (fig. 63), par des traits inclinés et un trait plus court, permet de faire nôtre la remarque de F. D'Errico, pour qui la forte flexibilité de la chaîne opératoire "est l'indice d'un intérêt pour le résultat final de la gravure» (D'Errico, 1994, p. 252). L'usage du bord du galet $\mathrm{n}^{\circ} 1148$ (fig. 63) pour des percussions n'est d'ailleurs pas une preuve de désacralisation, mais peut s'intégrer dans des processus cultuels divers. Quoi qu'il en soit, les travaux fondamentaux de F. D'Errico «dé-sanctuarisent» ces galets gravés, pour en faire une expression symbolique immédiate: le fait de découvrir de tels galets au beau milieu du locus dont le spectre fonctionnel est le plus varié des Chaloignes en constitue encore une preuve.

Même si ces galets sont d'ordinaire qualifiés d'Azilien, la démonstration restait à réaliser pour le nord-ouest de la France, à cause de contextes archéologiques médiocres qui autorisaient toutes les attributions. Dans le bassin inférieur et moyen de la Loire, trois sites ont livré des galets gravés: Chênambault (La Pommeraye, Maine-et-Loire ; Mornand, 1982, 1987, 1990 et 1992; Braguier et al., 1988; D’Errico, 1994; Bazin et al., 1995), le Clis (Guérande, LoireAtlantique; Gallais, 1984; Bazin et al., 1995) et La Motte (Montsoreau, Maine-et-Loire; Bazin et al., 1995). Sur le site paléolithique terminal de la Guichaumerie à Écouflant (Maine-et-Loire), M. Gruet avait découvert un galet au bord incisé, déposé aujourd'hui au Muséum d'histoire naturelle d'Angers (Gruet, 1938 et 1943; Siraudeau, Comte, 1998). Ce galet allongé en grès, de section ovale, est cassé en deux; l'extrémité subsistante mesure $35 \mathrm{~mm}$. Les incisions simples sont obliques sur les bords; un croisillon de traits courts couvre une des faces. Les galets des Chaloignes rencontrent des similitudes indéniables avec les pièces de Chênambault (La Pommeraye, Maine-et-Loire). Les huit galets découverts en surface sur ce site ont des identifiants de A à $\mathrm{H}$ (fig. 64 et 65). Le bord strié du $\mathrm{n}^{\circ} 1148$ des Chaloignes est identique à ceux des galets $\mathrm{B}$ (fig. 64, $\mathrm{n}^{\mathrm{o}} 4$ ) et $\mathrm{J}$, et surtout du galet $\mathrm{C}$ (fig. 64, $\mathrm{n}^{\circ}$ 6). Les stries parallèles du $\mathrm{n}^{\circ} 120$ (fig. 63) sont identiques à celles des galets $\mathrm{B}$ et $\mathrm{E}$ (fig. $65, \mathrm{n}^{\circ} 6$ ). Les lignes plus anarchiques du $\mathrm{n}^{\mathrm{o}} 87$ (fig. 62) sont décelables sur le galet $\mathrm{F}$ (fig. 64, $\mathrm{n}^{\mathrm{o}} 7$ ). Seuls les décors exubérants du galet $\mathrm{A}$ de Chênambault, sorte de treillis (fig. $65, \mathrm{n}^{\circ} 1$ ), ne trouvent pas de correspondances aux Chaloignes.

Les galets gravés découverts dans le couloir ligérien montrent quelques spécificités: alors que, dans l'Azilien, les traits sont le plus souvent sur une face, dans le val de Loire, les deux faces sont gravées. Par ailleurs, le système à deux séries parallèles opposées est inconnu en val de Loire (Thévenin, 1983; Bazin et al., 1995), ce qui distingue ces galets du style de Rochedane (Villars-sous-Dampjoux, Doubs), proposé par A. Thévenin, et largement répandu dans le sud-ouest de la France et le Jura. Il convient de noter que ces incisions de lignes parallèles sont courantes dans l'Azilien en général et permettent seulement de dégager un style «par défaut» sur cette aire géographique, que le faible nombre de découvertes rend fort provisoire. Totalement inséré dans la tradition azilienne, ce style s'oppose en revanche à des décors décrits récemment en Bretagne. Les pierres gravées découvertes en 

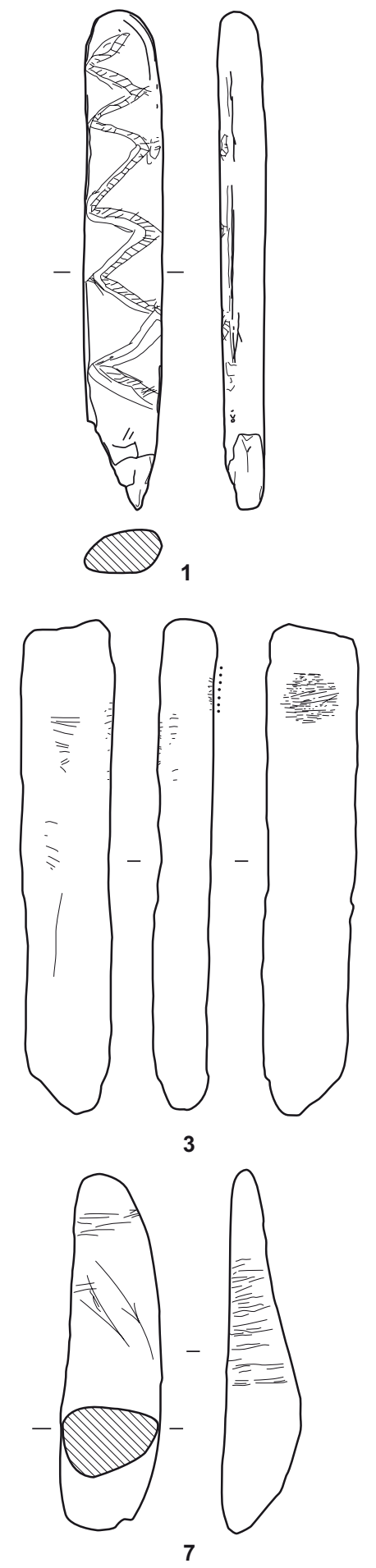
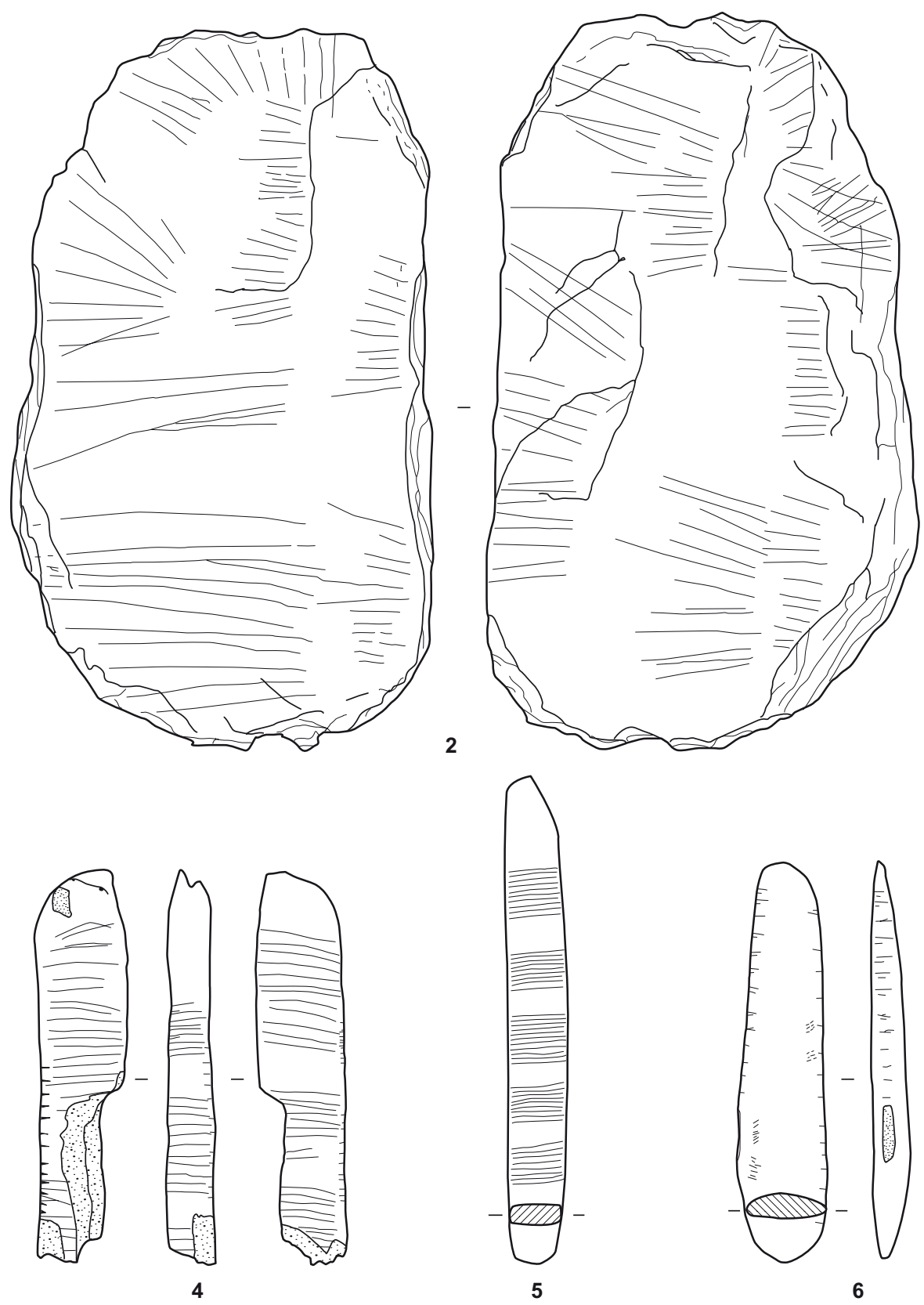

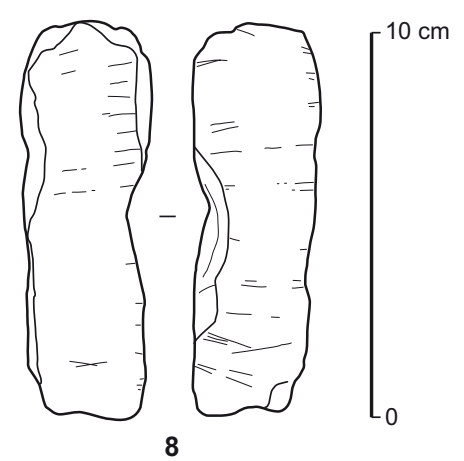

Fig. 64 - Planche synthétique des galets gravés de l'Ouest de la France. 1, Le Goërem-Kerbilaouet (Plonéour-Lanvern, Finistère), pierre $n^{\circ} 4$; 2 et 8 , Le Clis (Guérande, Loire-Atlantique); 3, galet $n^{\circ} 87$ des Chaloignes; 4-7, Chênambault (La Pommeraye, Maine-et-Loire), galets B, D, C et F. (dessins: L. Quesnel, CNRS UMR 6566). 

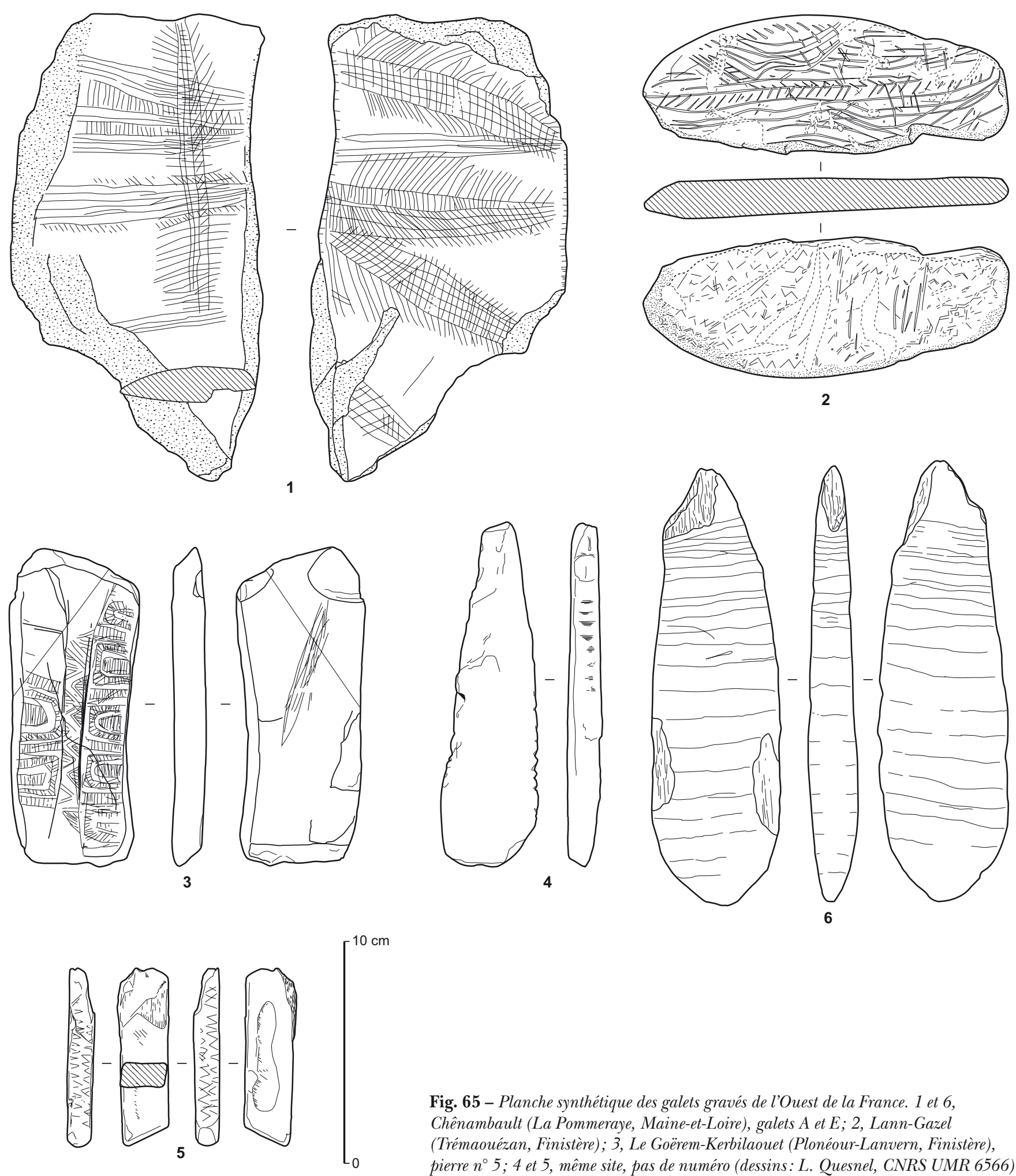

Fig. 65 - Planche synthétique des galets gravés de l'Ouest de la France. 1 et 6 , Chênambault (La Pommeraye, Maine-et-Loire), galets A et E; 2, Lann-Gazel (Trémaouézan, Finistère); 3, Le Goërem-Kerbilaouet (Plonéour-Lanvern, Finistère), pierre $n^{\circ}$ 5; 4 et 5, même site, pas de numéro (dessins: L. Quesnel, CNRS UMR 6566). 
surface d'un champ au Goarem Kerbilaouët en PlonéourLanvern (Finistère; Le Goffic, 1993) affichent des décors plus complexes, en lignes brisées ou en arceaux, plus proche des signes du Mésolithique final du Morbihan, découverts par exemple sur des outils en os à Téviec (Morbihan; Péquart et al., 1937) ou à Beg-er-Vil (Morbihan; Kayser, Bernier, 1988). Le galet gravé de Lann Gazel (Trémaouézan, Finistère; Le Goffic, 2001; fig. 65, $\mathrm{n}^{\circ}$ 2) a également une thématique fort différente du style ligérien, à base de lignes longitudinales sinueuses et de décors en chevrons. Il est associé pourtant à une industrie à pointes à dos courbe apparemment homogène et il est plus difficile de proposer une autre attribution chrono-culturelle que l'Azilien. Est-ce un premier pas vers la création d'un style symbolique ouest armoricain? Ces signes ne rencontrent pas de similitudes parmi les rares galets incisés d'Angleterre. Pour l'instant, il est préférable de rester prudent.

À la suite de travaux récents (Bazin et al., 1995), nous sommes alors conduits à définir un style culturel propre au cours inférieur et moyen de la Loire, lui-même totalement inséré dans le vaste corpus des galets gravés et peints aziliens. Le contexte de découverte des galets des Chaloignes permet d'intégrer avec certitude cet ensemble dans l'Azilien récent et non plus dans le Mésolithique ancien ou moyen comme cela avait été proposé antérieurement. Il faut cependant remarquer que les stries parallèles des bords de galet persisteraient dans le Sauveterrien de l'abri des Usclades à Nant (Aveyron; Maury, 1995) et, avec plus de réserve, à cause de l'absence de contexte stratigraphique, dans le Mésolithique moyen du Carpont à Sibiril (Finistère, Le Goffic, 1996).

G. M.

\section{UNITÉ ET DIVERSITÉ}

\section{DU TECHNO-COMPLEXE DES INDUSTRIES À POINTES À DOS COURBE DANS L'OUEST DE LA FRANCE}

\section{SITUATION GÉNÉRALE}

En France, l'apparition des pointes à dos courbe à la fin de la période paléolithique se fait sur un substrat culturel magdalénien. Selon A. Thévenin, elles pourraient apparaître en basse vallée du Rhône dans l'intervalle 1380012800 avant notre ère, dans le Dryas ancien, à l'interface entre le Bouvérien provençal de tradition épigravetienne et le Magdalénien (Thévenin, 1997). Dans cette optique, la diffusion vers le nord se ferait progressivement au sein même des industries magdaléniennes, jusque vers 12300 avant notre ère ou un peu après. Le techno-complexe des pointes à dos courbe prend réellement son essor à partir de ce moment. Dans l'Ouest, cette intrusion progressive se manifeste notamment dans le Magdalénien (couche 5) de l'abri-sous-roche du Bois Ragot (Gouex, Vienne), daté autour de 12600 BP, soit l'intervalle 13500-12400 avant notre ère (Chollet, Dujardin, 2005) (fig. 66). L'Azilien au sud de la Loire et le Federmesser autour de la Seine se partagent ensuite le territoire entre 12000 et 11000 avant notre ère (11800-10800 BP), durant tout l'interstade d'Alleröd, et peut-être au-delà. En quoi cette «azilianisation» est-elle liée aux modifications environnementales? L'adoption de l'arc est une des hypothèses fréquemment émises pour en rendre compte (Célérier, Moss, 1983; Fagnart, 1997), avec une chasse isolée en milieu supposé boisé qui est favorisée par cette arme légère, qui ne demande pas d'amples mouvements pour sa mise en œuvre. Pourtant, les analyses palynologiques de L. Visset et D. Barbier décrivent en Mayenne un environnement encore très ouvert pendant cet interstade (Barbier, 1999; Barbier, Visset, 2000). Dans le centre du Bassin parisien ou en Aquitaine, les analyses isotopiques réalisées sur les ossements témoigneraient aussi d'un prélèvement des ongulés dans un milieu ouvert (Bémilli, 2000; Drucker, Célérier, 2001). Cette modification technique n'est donc pas strictement corrélée aux changements environnementaux, mais doit plutôt être qualifiée d'invention qui va faciliter la chasse en milieu boisée (Rozoy, 1997).

Les faunes découvertes en association avec des pointes à dos courbe n'ont plus de caractères arctiques, et ce avant même le début de l'oscillation d'Alleröd. Dans le nord de la France comme en Aquitaine, la chasse aux grands mammifères concerne le cheval, l'aurochs, le cerf (Fagnart, Coudret, 2000; Bémilli, 2000; Bignon, Bodu, 2006), soit un spectre faunique caractéristique d'un milieu tempéré et de forêt claire. Il en va de même dans la couche $4 \mathrm{du}$ Bois-Ragot (Gouex, Vienne), attribuée à un Azilien ancien, où le cerf, le chevreuil et le sanglier sont chassés (Griggo, 2005). Notons que les suidés sont encore rares dans le nord de la France à cette période (Bridault, 1997). Fait plus rare, le lion est mentionné au Closeau à l'Azilien ancien (Rueil-Malmaison, Hauts-de-Seine; Bodu, Bémilli, 2000). Ce spectre de chasse élargi par rapport au Magdalénien sonne comme le prélude aux économies diversifiées du Mésolithique, dont les exemples les plus flagrants dans l'ouest de la France apparaissent à la fin de la période (Marchand, 2005). 


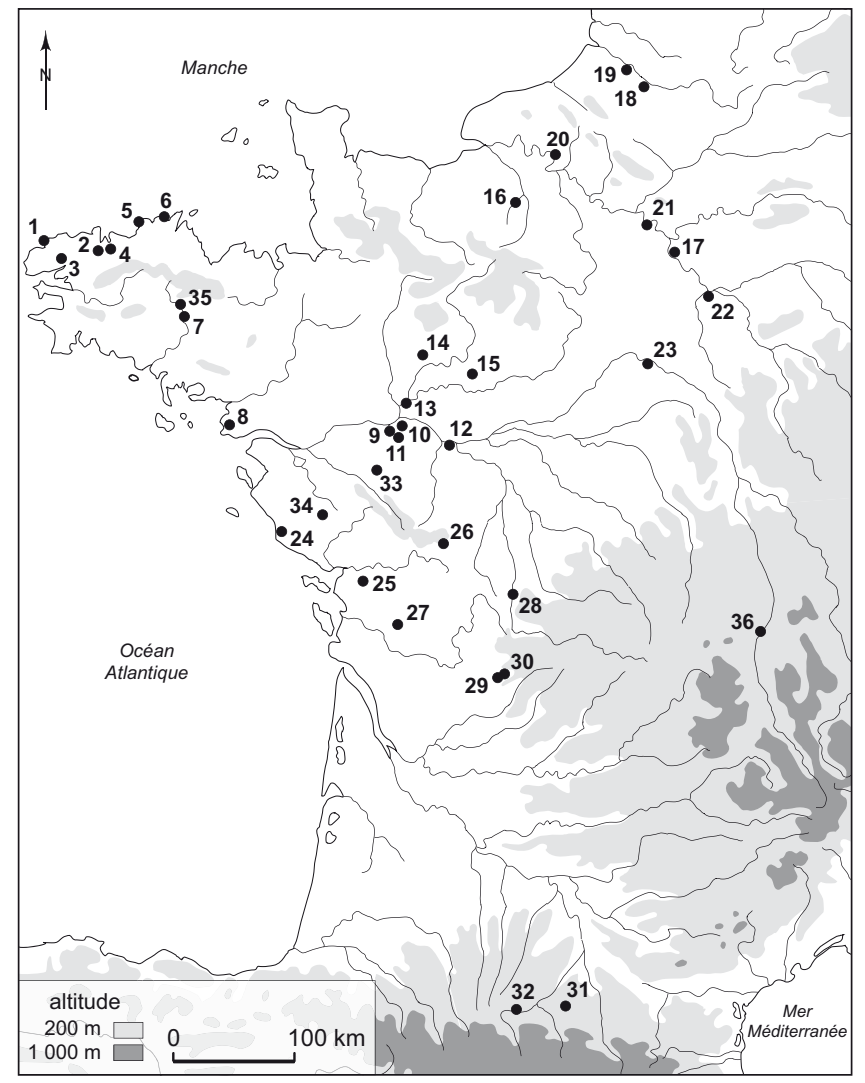

\section{ENQUÊTE EN PAYS DE LA LOIRE}

En Anjou, l'Azilien ne se manifeste qu'à la faveur de la découverte de pointes à dos courbe, immergées parmi des vestiges du Néolithique ou du Mésolithique (fig. 66). Lors de la prospection pédestre réalisée sur le tracé de l'A87, une pointe azilienne a été trouvée au sommet de la rive nord du Layon, au lieu-dit le Moulin-Brûlé (Beaulieu-sur-Layon, Maine-et-Loire). Lobjet isolé gisait en surface sur un léger replat couvert de vignes, en contrebas du sommet. Les sondages mécaniques ultérieurs, réalisés par Y. Viau, n’ont rien livré d'autre et il faut bien se résoudre à l'hypothèse d'une brève halte de chasse à proximité immédiate d'une voie de circulation logique sur ce coteau abrupt. Le plus éclatant de ces sites aziliens angevins - déjà mentionné plus haut - est celui de Chênambault à la Pommeraye (Maine-etLoire), situé au sud de la Loire, à une trentaine de kilomètres à l'ouest des Chaloignes (Mornand, 1982, 1987, 1990 et 1992; Braguier et al., 1988; Bazin et al., 1995). Il a livré huit galets de schistes gravés de fines incisions. Les ramassages de surface ont permis de recueillir plus de 20000 pièces, avec des composantes moustériennes, gravettiennes,
Fig. 66 - Localisations des sites à pointes à dos courbe mentionnés dans le texte. 1, Guennoc (Landéda, Finistère); 2, Roc'h Toul (Guiclan, Finistère); 3, Lann Gazel (Trémaouézan, Finistère); 4, Quillien (Le Cloitre-Saint-Thégonnec, Finistère); 5, Notenno (Trébeurden, Finistère); 6, Roc'h Glas (Penvenan, Finistère); 7, Le Perzo (Neuillac, Morbihan); 8, Le Clis (Guérande, Loire-Atlantique); 9, Chênambault (La Pommeraye, Maine-et-Loire); 10, Les Chaloignes (Mozé-surLouet, Maine-et-Loire); 11, Le Moulin-Brûlé (Beaulieu-sur-Layon, Maine-et-Loire); 12, La Motte (Montsoreau, Maine-et-Loire); 13, La Guichaumerie (Écouflant, Maine-et-Loire); 14, Grotte Rochefort (Thorigné-en-Charnie, Mayenne); 15, Létang du Cruchet (Ecommoy, Sarthe); 16, Ambenay (Eure); 17, Etiolles (Essonne); 18, La Vierge Catherine (Saleux, Somme); 19, Hangest-sur-Somme (Somme); 20: Gouy (Seine-Maritime); 21, Le Closeau (Rueil-Malmaison, Hauts-de-Seine); 22, Pincevent (Seine-et-Marne); 23, Autry-le-Châtel (Montargis, Loiret); 24, Coёx/L'Aiguillon-sur-Vie (Vendée); 25, La Grange (Surgères, Charente-Maritime); 26, La Bourronnière (Ménigoute, Deux-Sèvres); 27, Piphrez (Grandjean, Charente-Maritime); 28, Le Bois-Ragot (Gouëx, Vienne); 29, Rochereil (Grand-Brassac, Dordogne); 30, Le Pont d'Ambon (Bourdeilles, Dordogne); 31, Le Mas d'Azil (Ariège); 32, La Tourasse (Saint-Martory, Haute-Garonne); 33, Létang de Péronne (Chanteloup, Maine-et-Loire); 34, La Girardière (La Ferrière, Vendée); 35, L'Anse de Landroannec (Mûr-de-Bretagne, Morbihan); 36, Champ-Chalatras (Les Martres-d'Artière, Puy-de-Dôme) (dessin: G. Marchand, CNRS UMR 6566).

aziliennes, mésolithiques et néolithique final! L'attribution chronologique au Mésolithique des galets gravés (Bazin et al., 1995) est contestable, mais s'explique sans peine par l'abondance sur ce site de la composante d'une phase moyenne. Il y a pourtant à Chênambault quelques pointes à dos courbes, que l'exemple du locus 11 des Chaloignes nous incite désormais à relier aux galets gravés.

$\mathrm{Au}$ sud du département du Maine-et-Loire, l'Étang de Péronne à Chanteloup-les-Bois (Maine-et-Loire) est un point important pour la compréhension de l'Azilien de l'Ouest. Le site a été découvert par M. Goudé sur la rive d'un étang artificiel, et prospecté durant les années 1990 sur une petite surface (Marchand et al., 2004). Rien ne distingue topographiquement cet endroit, sinon une source pérenne dans un vallon très faiblement imprimé. Parmi les 697 pièces mises au jour, un poignard et quelques armatures microlithiques viennent signaler un mélange de collections, dû au prospecteur, mais bien cerné lors de cette étude. La rareté des pièces brûlées, l'absence de patine et un grand nombre de cassures postérieures au débitage (double patine) sont à mentionner, de même qu'une grande diversité des matériaux. À côté des silex blonds, issus de 
galets roulés qui comptent pour plus de la moitié des pièces, on décompte quatorze types de silex à cortex brut $(25,1 \%)$, un quartzite marron à la texture extrêmement fine (Montbert? 9,9\%) et quelques fragments d'un jaspe orange. Bon nombre de ces matières, comme le silex roulé issu des terrasses de la Loire ou le silex noir issu du niveau Turonien (tuffeau), sont aussi présentes aux Chaloignes, avec ici des distances d'approvisionnement supérieures à $30 \mathrm{~km}$. Il faut noter que les terrasses de la Loire sont plus proches de l'étang de Péronne que les niveaux à silex du Bassin parisien. Les lames aux nervures irrégulières, tirées à la percussion directe dure, sont la production dominante, avec de nets indices d'un débitage bipolaire sur une même table. Le quartzite a porté une production laminaire bien menée avec un percuteur en pierre tendre (grès); l'entretien est plus important que sur les autres matières, avec notamment de nombreuses tablettes de ravivage et des néo-crêtes partielles. Les éclats, qui comptent pour $72 \%$ des enlèvements bruts, sont produits par des méthodes bipolaires ou unipolaires, à la percussion directe dure. Enfin, la percussion bipolaire sur enclume apparaît parfois, d'une part à des fins de production de support, d'autre part dans des procédés de reconversion de certains outils. La structure générale de l'outillage et le style de la production sont comparables à ce que l'on connaît aux Chaloignes. L'outillage aménagé est dominé par les grattoirs sur éclat, grossièrement circulaires et parfois à front surbaissé. Les burins sont à peine moins nombreux, avec une grande diversité morphologique liée aux supports transformés, épais et irréguliers. Les troncatures et les bords abattus sur éclats ou sur lame sont en nombre sensiblement égaux et ils affectent des supports très divers. Parmi les armatures, on remarquera une monopointe azilienne en quartzite, avec une petite troncature basale (pointe de Grundy). Les fragments de pièce à dos appartiennent probablement dans un cas à une pointe azilienne, mais dans les autres cas à des lamelles à dos plus étroites. En conclusion, le site de l'Étang de Péronne fournit les preuves d'une occupation azilienne bien caractérisée, avec une industrie lithique similaire à bien des égards à celle des Chaloignes, tant dans l'outillage que le débitage ou l'économie des matières premières. Cette dernière est placée sous le sceau de la diversité, probablement à cause de la position intermédiaire de cet habitat, entre la Loire et le seuil du Poitou.

La fouille de sauvetage réalisée à l'Étang du Cruchet (Écommoy, Sarthe) vient également apporter des références nouvelles pour les industries à pointes à dos courbe des Pays de la Loire. Ce site a été exploré sur le tracé de l'A28 en octobre 1997, sous la direction de J. Musch (Musch, 1997). Le sédiment sableux n'a pas permis la conservation de l'os. Le faible nombre de pièces mises au jour (12 outils et 233 déchets de débitage) indique une occupation de courte durée, qui se présentait sous la forme d'un locus entièrement compris dans la surface de fouille restreinte $\left(44 \mathrm{~m}^{2}\right)$. L'industrie lithique présente de nombreux points communs avec celle des Chaloignes. Les deux pointes à dos courbe entières et le fragment pourraient être des bipointes. On doit encore noter la présence de deux grandes lames à bord abattu courbe, classées indûment parmi les armatures, de grattoirs courts sur éclats, parfois unguiformes, et de deux burins peu standardisés. Les grandes dimensions de l'industrie sont des caractères marquants, qui diffèrent notablement des industries des Chaloignes; les deux pointes ne trouvent comparaison qu'avec les plus grands exemplaires des Chaloignes. Une origine différente de la matière première est en cause, puisque, de toutes les façons, le recours aux galets de Loire ne permettait pas d'obtenir de tels modules; d'ailleurs, parmi d'autres pièces des Chaloignes, un couteau à dos du locus 11 de grandes dimensions est en silex exogène. La mise en œuvre de crêtes sur le site sarthois répond au besoin d'aménager les volumes à débiter, opération sans intérêt pour les galets roulés des Chaloignes, mais qui constitue un dernier argument en faveur de ce déterminisme. Par ailleurs, la méthode bipolaire de production laminaire n'est apparemment pas présente à l'Étang du Cruchet, ce qui pourrait avoir une forte signification culturelle. Il faut noter que le lien entre l'abondance de la matière première et les dimensions des pièces n'est pas systématique, comme on le voit dans le Mésolithique moyen, et il convient de l'examiner pour chaque système technique. Il semble opérant dans le cas des industries à dos courbes, comme l'a noté J.-P. Fagnart en constatant le «gigantisme» des pièces dans le bassin de la Somme ou en Haute-Normandie (Fagnart, 1997, p. 224).

En Mayenne, l'enquête exemplaire menée par M. Allard parmi toutes les anciennes collections des fouilles des grottes de Thorigné-en-Charnie et de Saint-Pierre-surErve a révélé l'existence de quelques pièces aziliennes, sans contexte stratigraphique (Allard, Gruet, 1976; Allard, 1983). Elles seraient issues de la grotte Rochefort. Il y a quelques pointes aziliennes, des grattoirs courts et un harpon à double rang de barbelures, portant apparemment deux perforations basales sur la base de la hampe. En Vendée, un inventaire récent réalisé par G. Gouraud, J.-M. Jauneau, O. Gandriau et C. Dugast (Gouraud et al., 
1998; Jauneau et al., 2000) a permis de garnir le sud du Massif armoricain de pointes aziliennes, démontrant par là-même le potentiel de ce département. Ces éléments sont issus de tris parmi des collections mélangées. Des parcelles prospectées sur les communes voisines de Coëx et de l'Aiguillon-sur-Vie, près des Sables-d'Olonne, ont livré tout particulièrement des pointes à bord abattu courbe et des pointes de Malaurie. La série la plus homogène est dans l'immédiat celle de la Girardière (La Ferrière, Vendée). En Loire-Atlantique également, sont mentionnées des découvertes sporadiques de pointes à dos courbe près du Lac de Grand-lieu ou en presqu'île de Guérande (Collectif, 1984). Dans cette dernière, on doit relever la présence de galets gravés sur le site du Clis (Gallais, 1984; Bazin et al., 1995). Encore une fois, un tri typologique a été nécessaire parmi des collections de surface très hétérogènes.

Conclure sur ces éléments épars est une gageure. L'occupation des Pays de la Loire pendant la fin du Tardiglaciaire est évidente, mais il est presque impossible de dépasser le niveau d'un inventaire par présence/ absence, alors que la position charnière de cette région appelait de longs commentaires sur les influx culturels et notamment la pseudo opposition Azilien/Ferdermesser. Notons cependant quelques points positifs, comme la présence d'un harpon azilien typique en Mayenne, qui vient confirmer encore un peu plus que l'Azilien des Pays de la Loire est intensément «normal», évalué à l'aune du reste des productions sur le territoire français. Tout reste maintenant à écrire sur les éventuels aspects particuliers, voire les faciès de l'Azilien de l'Ouest.

\section{ENQUÊTE EN POITOU-CHARENTES}

La grotte du Bois-Ragot à Gouex (Vienne) est une des principales références pour observer l'évolution des industries lithiques et osseuses tardiglaciaires dans l'ouest de la France, même si, en un tel contexte archéologique, les déplacements d'objets entre couches doivent être pris en compte. Elle a été fouillée entre 1978 et 1989 par A. Chollet et P. Boutin (Chollet, 1990; Célérier, et al., 1997; Chollet, Dujardin, 2005). Un niveau magdalénien (niveau 5) et deux niveaux aziliens (niveaux 4 et 3) ont été reconnus. Il est aisé de corréler le niveau 3B de Pont d'Ambon (Bourdeilles, Dordogne; Célérier, 1994 et 1998) et le niveau 4 du Bois-Ragot, qui présentent des caractères si proches; ils témoignent d'un Azilien ancien à bipointes. Le débitage laminaire est encore fort régulier; les supports allongés obtenus permettent de réaliser des grattoirs et des burins de belle venue. Le niveau 3 du Bois-Ragot, fort d'environ 3000 éléments lithiques, présente de très grandes similitudes avec les Chaloignes. La récente étude réalisée par B. Valentin montre une production bipolaire avec une alternance assez lente des séquences de débitage à partir de l'un puis l'autre plan de frappe, avec une mise en forme modeste du volume à débiter, une abrasion des corniches peu insistante et un débitage à la pierre tendre (Valentin, Hantaï, 2005). Le facettage des talons de lame existe dans les niveaux aziliens du Bois-Ragot, mais en proportions moindres qu'aux Chaloignes. Parmi les outils communs aménagés, les grattoirs sur éclat sont dominants, devant les burins. Les armatures sont principalement des monopointes à dos courbe; les lamelles à dos nombreuses autrefois signalées (Chollet, 1990) seraient soit des fragments de pointes aziliennes, soit des armatures issues d'une occupation mésolithique résiduelle au-dessus du niveau azilien (Valentin, Hantaï 2005). Il s'agissait du principal obstacle à un fort rapprochement entre le site d'Anjou et celui du Poitou, désormais levé. L'analyse lithologique de P. Fouéré au Bois-Ragot montre que les silex locaux (Bajocien gris) furent principalement débités, à la différence des niveaux antérieurs où l'économie d'acquisition profitait de plus larges espaces (Fouéré, 2005). Cette restriction des territoires d'acquisition des matières premières a été également mentionnée aux Chaloignes lors de notre étude; pour nous, cette information signe l'entrée dans les comportements techniques propres au Mésolithique, en lien avec des systèmes techniques moins exigeants.

Hormis ce site phare, de nombreuses trouvailles attestent que la Charente et le seuil du Poitou ont été le siège de communautés aziliennes (Perpère, 1976; Joussaume, Pautreau, 1990; Foucher, San Juan, 1994). Des fouilles à la grotte du Quéroy à Chazelles (Charente; Tournepiche, 1986) ou sur le site alluvial de La Grange à Surgères (CharenteMaritime; Marchand et al., 2000; Laporte et al., 2000) ont livré à chaque fois des vestiges aziliens, sans que des niveaux bien caractérisés puissent être diagnostiqués. L'abri-sousroche de Piphrez (Grandjean, Charente-maritime) n'est connu que par des ramassages postérieurs à sa destruction lors de travaux autoroutiers, qui ont donné quelques pièces. Plus au nord, le ramassage de surface sur le site de la Bouronnière à Ménigoute (Deux-Sèvres) offre un relais intéressant pour montrer le continuum de l'occupation azilienne des Chaloignes à l'Aquitaine (Joussaume, Pautreau, 1990). 


\section{ENQUÊTE EN BRETAGNE}

À défaut de fouilles récentes, la Bretagne tient une place encore marginale dans cette recherche sur les cultures du Tardiglaciaire du nord de la France. Mais les inventaires récents sont en train de modifier les perspectives sur le Tardiglaciaire dans cette péninsule (Marchand et al., 2004). Deux sites fouillés dans le Finistère témoignent de la présence azilienne: la grotte de Roc'h Toul à Guiclan et Enez Guennoc à Landéda. Le premier a été exploré en 1868 et 1869 par le Docteur Le Hir, puis par C. Bénard dit le Pontois et enfin par E. Morel; le matériel a été étudié par G. Laplace (Laplace-Jauretche, 1957), puis par J.-L. Monnier (Giot et al., 1977; Gouletquer, Monnier, 1976; Monnier, 1980). L'industrie lithique de Enez Guennoc a été découverte lors des fouilles de P. R. Giot, dans deux zones de fouille principales. Elle se plaçait dans un niveau sousjacent à des cairns néolithiques (Monnier, 1980). Il est de tradition, depuis les recherches de J.-L. Monnier, de placer Roc'h Toul avant Enez Guennoc; de fait à Roc'h Toul, les bipointes, le fort taux de burins ou les grattoirs sur lames nous orientent vers une phase assez ancienne de l'Azilien (pour autant qu'une fouille aussi ancienne nous permette ce type de réflexion), tandis que des microlithes nongéométriques d'Enez Guennoc indiqueraient une phase de transition (ou un niveau mélangé). Le taux de denticulés en Bretagne nous éloigne des industries des Chaloignes, mais les éléments communs sont très nombreux, d'un point de vue typologique, et probablement aussi technologique, puisque l'on reconnaît sur les planches de Roc'h Toul des nucléus à lames dont l'exploitation se fait à partir de deux plans de frappe opposés.

Un inventaire collectif récent montre que le nombre de stations est considérablement plus élevé (Marchand et al., 2004). Le résultat des prospections intensives menées en Finistère au cours des années 1990 par P. Gouletquer et son équipe a été notamment réévalué. Une quarantaine de sites est d'ores et déjà connus, dont plusieurs sont des ensembles majeurs:

- Lann Gazel (Trémaouézan, Finistère; Le Goffic, 2001),

- Quillien (Le Cloître Saint-Thégonnec, Finistère; Gouletquer, Léopold, 1991),

- Runigou/Notenno (Trébeurden, Côtes-d'Armor; Chevalier, 1999),

- Roc'h Glas (Penvénan, Côtes-d'Armor; Chevalier, 1999), - Le Perzo (Neuillac, Morbihan; inédit G. Tournay),

- L'anse de Landroannec (Mûr-de-Bretagne, Côtes-d'Armor, inédit $\mathrm{O}$. Kayser et G. Tournay).
Tous sont contemporains de la phase à monopointes, soit un Azilien récent. Les pointes à dos anguleux n'apparaissent que dans le nord du Finistère, sur le site de Lann Gazel (Le Goffic, 2001), en accompagnement de monopointes et bipointes aziliennes. Cette association rappelle les assemblages à "penknife points» (dites aussi pointe de Grundy) présents dans le sud de l'Angleterre. Ils sont postérieurs au Creswellien et contemporains de l'Alleröd (Barton, Roberts, 1997). Ces rapprochements incitent à considérer autrement la géographie humaine autour de l'embouchure du vaste estuaire de la «Manche», avec peut-être un fonctionnement territorial différent du reste de la région. M. Le Goffic a d'ailleurs bien montré que le silex utilisé à Lann Gazel provenait directement d'affleurements datés du Crétacé, rendus aujourd'hui inaccessibles par la transgression flandrienne (Le Goffic, 2001).

\section{LES PLUS PROCHES RÉFÉRENCES FIABLES DES CHALOIGNES}

Les stations de références de bonne qualité sont situées dans le Bassin parisien et dans le Bassin aquitain (fig. 66). L'attribution du site des Chaloignes à l'Azilien impose en premier lieu une référence au site éponyme des Pyrénées, le Mas-d'Azil (Ariège), et à son alter ego, la Tourasse (SainteMartory, Haute-Garonne), pris comme référence par G. Mortillet (Orliac, 1973 et 1975). Mais cet azilien classique est probablement plus récent (Dryas récent?) que dans les régions plus septentrionales (ibid.; Rozoy, 1978; Marchand, 1991). Soulignons seulement la médiocrité du débitage, avec le recours à la percussion bipolaire sur enclume, ainsi que le style des pointes aziliennes typiques, assez symétriques et proches des pointes d'Istres. Cela confère à l'Azilien «classique» des Pyrénées un statut un peu à part, peut-être un faciès à définir plus précisément.

Les abris-sous-roche du Bois-Ragot à Gouex (Vienne) et du Pont d'Ambon à Bourdeilles (Dordogne) constituent les bases de la réflexion sur l'Azilien dans le Poitou et au nord du Bassin aquitain. Les caractères du premier ont été détaillés plus haut. Le second comporte un niveau magdalénien avec déjà quelques pointes à dos (niveau 4), trois niveaux aziliens (niveaux 3B, 3A et 3) et un niveau laborien (niveau 2) (Célérier et al., 1993 et 1997; Célérier, 1998). Pour faire bref, l'Azilien initial du niveau 3B comporte des bipointes à dos, quelques rares lamelles à dos, des grattoirs courts sur lame. Le débitage laminaire est régulier, avec notamment une abrasion soigneuse des corniches. L'azilien dit «récent» des niveaux $3 \mathrm{~A}$ et 3 montre des changements techniques assez 
marqués, avec une domination des pointes aziliennes à base rétrécie, des grattoirs courts réalisés sur éclat et un débitage nettement moins soigné, avec des corniches non-abrasées et l'usage de la percussion directe dure. Le niveau laborien (niveau 2) qui coiffe cette séquence est proche de celui de la Borie-del-Rey (Blanquefort-sur-Briolance, Lot-et-Garonne; Coulonges, 1963; Le Tensorer, 1981); il est frappant d'y constater le retour à la forte importation de silex allochtone pour un débitage laminaire de meilleur niveau technique. À quelques centaines de mètres en aval de la Dronne, la grotte de Rochereil (Grand-Brassac, Dordogne; Jude, 1960) apparaît comme le jumeau maudit de Pont d'Ambon: la couche III, épaisse de 1,80 m, laisse entrevoir également le passage du Magdalénien VI à l'Azilien, mais il faut bien admettre que la piètre qualité des fouilles de P. E. Jude ne permet pas de séparer les probables séquences chronologiques que la présence de bipointes aziliennes et de pointes de Malaurie démontre pourtant.

Dans le centre du Bassin parisien et dans la vallée de la Somme, les travaux récents, tant universitaires (Fagnart, 1997; Valentin, 1995) que liés à l'archéologie préventive (Bodu, 1995; Bodu, Valentin, 1997), ont notablement fait évoluer les problématiques. Les sites de référence les plus proches des Chaloignes sont Gouy (Seine-Maritime) et le Closeau (Rueil-Malmaison, Hauts-de-Seine). Une rupture technique nette est observée entre une phase ancienne et une phase récente des groupes à Federmesser, qui semble être une constante dans les industries à pointes à dos courbe de l'Europe de l'Ouest (Valentin 2000). Dans le niveau inférieur du Closeau, le débitage est régulier, avec des crêtes antérieures voire postérieures; les plans de frappe sont lisses et abrasés pour une percussion à la pierre tendre (Bodu, 2000b). L'outillage comprend des bipointes, des burins et des grattoirs longs. On retrouve ces caractères dans la grotte du Cheval à Gouy et dans le niveau inférieur d'Hangest-sur-Somme III 1 (Somme; Fagnart, 1997). Cette phase est nettement antérieure au réchauffement de l'Alleröd. Une phase moyenne encore conjecturale pourrait être détectée à Ambenay (Eure; Fosse et al., 1997) ou dans le niveau III 2 de Pincevent (Seine-etMarne; Bodu, Valentin, 1997); elle se caractériserait par une simplification du débitage. Dans l'outillage, notons la domination des monopointes à dos courbe et des lamelles à dos. La phase tardive des groupes à Federmesser du Bassin parisien est mieux connue; elle a été distinguée au Closeau (niveau supérieur) et sur le site de la Vierge Catherine à Saleux (Somme; Fagnart, 1997). Au Closeau, la mise en forme des volumes est inexistante, la percussion directe dure est appliquée sans finesse. Les monopointes à dos courbe sont irrégulières, les grattoirs sont courts. À Saleux, le débitage est un peu plus régulier, mais avec une mise en forme toujours inexistante, une abrasion parcimonieuse des corniches et un facettage fréquent des plans de frappe. Les monopointes aziliennes sont très nombreuses à la différence des lamelles à dos. Comme souvent dans la Somme, les burins sur troncature dominent sur les burins dièdres. Pour conclure sur cette aire géographique, une tripartition de l'Azilien est de mise comme dans le sud-ouest, avec une phase ancienne au tout début de l'Alleröd ou un peu avant, une phase récente en nette rupture technologique (Valentin, 1995 et 1999), datée de la seconde partie de cet interstade, et enfin un développement du faciès à pointes de Malaurie (ou Laborien), juste avant le Dryas récent. L'existence d'une phase entre le stade à bipointes et le stade à monopointes, suggérée en particulier par P. Bodu (Bodu, Valentin, 1997; Bodu, 2000a), est encore à confirmer.

Les niveaux aziliens récents de Pont d'Ambon (niveaux 3 et 3A) et du Bois-Ragot (niveau 3) sont donc les références les plus évidentes des Chaloignes. Parmi les caractères particuliers des industries de ce site, on peut retenir surtout des absences, comme les pièces esquillées et surtout les lamelles à dos (les fragments à dos semblent être ici tous rapportables aux pointes dont ils partagent le module et il n'y a pas de production spécifique de supports étroits). Il convient de noter également l'extrême rareté des denticulés. Il n'y a pas aux Chaloignes d'éléments typologiques indiscutablement «hors normes» dans le techno-complexe des industries à dos courbe. La base rétrécie des pointes aziliennes, commune à Pont d'Ambon et aux Chaloignes, a servi à définir la pointe de Grundy. Il s'agit très probablement d'une régularisation de supports assez peu calibrés, liée à l'emmanchement, et il est difficile de bâtir un faciès chrono-culturel sur ce seul point. Les dates par le radiocarbone de cet Azilien récent couvrent un large intervalle, avec de grandes incohérences liées notamment à un effet de plateau dans la courbe de calibration. Dans la Somme, les industries à monopointes se placent à l'Alleröd, tandis qu'au Closeau le niveau supérieur couvre le début du Dryas récent. A Pont d'Ambon, ces datations sont à la charnière avec le Préboréal. Dans la couche 4 du Bois-Ragot (Azilien à bipointe, antérieur au Chaloignes), une date donne l'intervalle 11900-11500 avant notre ère (Chollet, Dujardin, 2005). L'existence d'un gradient évolutif est possible, mais on observe un télescopage avec le Laborien, qui reste à expliquer. On peut, sur les bases définies au Closeau, proposer pour l'Azilien des Chaloignes une plage chrono- 
logique autour de 10800 BP (vers 11200-10600 avant notre ère), soit à la fin de l'Alleröd.

La position des Pays de la Loire entre l'aire azilienne et l'aire Federmesser nous oblige à prendre parti dans l'opposition entre ces deux cultures. Or, à partir des comparaisons effectuées entre l'Étang du Cruchet - qualifié de Federmesser - et les Chaloignes - qualifié d'Azilien -, il n'est pas possible d'établir de différences majeures dans la composition des industries. Les dimensions sont plus importantes dans la Sarthe, mais on a vu l'impact de la matière première sur la dimension des produits. Cette distinction recouvre essentiellement un vocabulaire d'attente; il ne convient pas d'opposer ces deux prétendues cultures qui diffèrent à partir de quelques éléments seulement (Bodu, Valentin, 1997). La grande homogénéité du phénomène d'azilianisation en Europe de l'Ouest doit être réaffirmée à l'issue de cet examen de la bibliographie et le site des Chaloignes vient encore confirmer cette observation.

\section{ORIGINE ET DEVENIR DES INDUSTRIES AZILIENNES SUR LE MASSIF ARMORICAIN}

Les ensembles lithiques attribués antérieurement au Magdalénien dans la région (Allard, Guyot, 1972; Allard, 1982; Gruet, Jaouen, 1957) étaient notamment caractérisés par des pointes à dos rectiligne. La plupart de ces assemblages ont été récemment placés après l'Azilien (Marchand et al., 2004; Marchand, 2008), par comparaison avec les stratigraphies de la Borie-Del-Rey (Blanquefort-sur-Briolance, Lot-et-Garonne; Coulonges, 1963; Le Tensorer, 1981) et dans une moindre mesure avec le niveau A4 de Rochedane (Villars-sous-Dampjoux, Doubs; Thévenin, 1982).

Il reste malgré tout quelques éléments de réflexion concernant les groupes antérieurs à l'Azilien: ainsi, dans la vallée de l'Erve en Mayenne, la grotte de la Bigotte, qui a livré des éléments magdaléniens dans des fouilles anciennes (Allard, Gruet, 1976; Allard, 1983). Il s'agit d'un lot de grattoirs sur grandes lames plates, un burin à bec de perroquet et une sagaie à section carrée et à base en double biseau. Ces données ponctuelles et faiblement exploitées empêchent néanmoins de considérer le degré d'occupation dans l'ouest de la France au début du Tardiglaciaire. La rareté des indices de la présence magdalénienne s'explique au moins partiellement par la rareté du silex, la mauvaise conservation des vestiges dans des contextes à faible puissance sédimentaire et peut-être le mauvais classement d'industries que l'on ne sait pas encore bien caractériser. Mais la multiplication des sites et indices de sites aziliens entraîne immanquablement une impression de forte augmentation de la population au cours de l'Alleröd. Les recherches à venir ne pourront éviter de traiter cette question fondamentale pour l'histoire de l'occupation humaine sur le Massif armoricain.

Que se passe-t-il après l'Azilien? Dans le sud-ouest (Coulonges, 1963; Le Tensorer, 1981; Thévenin, 1997), dans le Bassin parisien (Bodu, 2000a) ou plus loin, en Auvergne (Pasty et al., 2002), le Laborien correspond à la fin du cycle des industries à pointes à dos courbe, avec encore une forte empreinte technique azilienne. Le débitage laminaire gagne en qualité; les pointes de Malaurie tendent à supplanter les monopointes aziliennes. Les dates proposées vont du début du Dryas récent (pour le Closeau, Rueil-Malmaison, Hautsde-Seine) au début du Préboréal (pour Pont-d'Ambon et Champ-Chalatras, les Martres-d'Artière, Puy-de-Dôme). En Pays-de-la-Loire, seul le site de surface de la Guichaumerie (Écouflant, Maine-et-Loire; Gruet, 1938 et 1943; Marchand et al., 2004), étudié récemment par N. Naudinot, pourrait témoigner de cette phase, mais les associations entre les objets sont impossibles dans un assemblage disparate et trié lors de la prospection.

Dans le nord de la France, les faciès à lames mâchurées de type belloisien pourraient succéder à cette dernière phase du techno-complexe azilien. Ils sont identifiés dans le Bassin parisien et en Angleterre, avec des dates contemporaines de la fin de la glaciation ou du début de l'Holocène (Bodu, Valentin, 1997). Selon une hypothèse émise par B. Valentin (Valentin, 1995 et 1999), il pourrait s'agir de faciès techniques accompagnant les industries à pointes à dos rectiligne du type les Blanchères (La Boissière-École, Yvelines; Schmider, 1971; Rozoy, 1978). Dans le sud-ouest de la France, au tout début du Préboréal, le Laborien se transforme en Épilaborien, avec une apparition de lamelles à dos appointées et de micro-pointes aziliennes (Le Tensorer, 1981). Dans l'ouest, le site du Camp d'Auvours (Saint Mars-la-Brière, Sarthe; Allard, Guyot, 1972; Allard, 1982) et de la Vigie Romaine (Le Croisic, Loire-Atlantique; Sicard-Marchand et al., 2004) représenteraient cette phase, avec notamment un débitage laminaire régulier, des pointes à troncature rectiligne et des bitroncatures symétriques.

Pour ambigus qu'ils soient, les éléments découverts sur le Massif armoricain interviennent donc dans ce débat de manière non-marginale, pour peu que l'on se risque à réinterpréter les attributions culturelles originelles.

G. M. 


\section{PERSPECTIVES ÉCONOMIQUES SUR L'OCCUPATION AZILIENNE DES CHALOIGNES}

\section{CLIMAT ET ENVIRONNEMENT: UNE APPROCHE INDIRECTE}

L'occupation de la cuvette des Chaloignes à la fin de l'interstade d'Alleröd est fort probable, les analogies typologiques et technologiques ne laissent guère de doute à ce sujet, si bien sûr on ne pose pas l'hypothèse d'une exception culturelle angevine que la géographie expliquerait mal! Les analyses géomorphologiques et micromorphologiques vont d'ailleurs dans le sens d'une contemporanéité avec une amélioration climatique comprise entre deux périodes plus froides. Les données encore hétéroclites recueillies dans l'ouest ne permettent certainement pas de dresser de tableau cohérent de l'environnement au Tardiglaciaire. Des travaux récents sur trois tourbières du nord de la Mayenne nous laissent cependant esquisser quelques traits de la végétation à cette période (Barbier, 1999; Barbier, Visset, 2000). De manière générale en France, la clémence du climat à l'Alleröd, proche de l'actuel mais avec une plus grande instabilité, permet l'installation d'une forêt dominée par les bouleaux, les pins et les saules en zones humides. En nord-Mayenne, les données diffèrent quelque peu du modèle français avec une domination apparente d'une steppe à Poacées. Les températures semblent fraîches et le fort taux d'humidité entraîne les comparaisons vers les Îles britanniques. Pour mémoire, le refroidissement du Dryas récent est également enregistré, mais il semble modéré et de courte durée. Il serait pourtant responsable aux Chaloignes d'une phase érosive de certains niveaux sus-jacents à l'Azilien.

Dès l'Azilien ancien, la faune tempérée forestière domine sans partage sur le territoire français; les espèces arctiques ont déserté les lieux dès le milieu de l'interstade de Bölling. Si l'on étend au Massif armoricain les observations obtenues dans le Bassin parisien ou dans le Poitou, on peut imaginer un spectre de chasse assez ouvert, qui comprend potentiellement tous les grands mammifères de l'Europe tempérée.

\section{LE CHOIX TOPOGRAPHIQUE COMME PREMIER INDICE DE LA STRUCTURE ÉCONOMIQUE}

L'absence de restes osseux est évidemment un frein à l'analyse économique d'une société de chasse. Pourtant, à moins de renoncer définitivement à comprendre les temps passés sur cette aire géographique, le raisonnement archéologique doit se satisfaire de données indirectes. Dans ce registre, l'implantation topographique de l'habitat est un premier élément déterminé, parmi d'autres facteurs, par des choix économiques, à savoir un ensemble de paramètres jugés favorables par les hommes pour inciter à de fréquents retours au même endroit au cours du cycle de mobilité annuel. Lorsque l'on fait varier les échelles spatiales des hommes de l'Azilien, quelques éléments structurants semblent apparaître.

Dans un rayon de $2 \mathrm{~km}$ autour du site, se trouvent, au nord, la confluence entre la vallée de la Loire et celle de l'Aubance (l'actuelle embouchure se trouve à plusieurs kilomètres en aval, mais l'Aubance en partie terminale emprunte la vallée de la Loire), et à l'est, le ruisseau des Jonchères, dont le cours est parallèle à celui qui draine les Chaloignes (fig. 2). Cette même distance permet d'atteindre au sud le sommet du versant, qui domine depuis ses $92 \mathrm{~m}$ N.G.F. toute cette partie de la vallée de la Loire jusqu'à Angers (fig. 3). Le site des Chaloignes a donc une position intermédiaire entre le fleuve et le sommet du versant exposé au nord du couple Loire/Aubance. Un rayon de $5 \mathrm{~km}$, soit environ une heure de marche en terrain découvert, permet d'intégrer un vaste territoire bordé au nord et à l'ouest par la Loire, et au sud par le ruisseau de la Planche, particulièrement encaissé. Notons que l'embouchure de la Maine est située à $5 \mathrm{~km}$ et demi au nord-ouest du site. L'environnement immédiat des hommes des Chaloignes est fortement marqué par le fleuve, avec $10 \mathrm{~km}$ de rives accessibles rapidement, et par ses nombreux affluents. On sait que ses berges étaient assidûment fréquentées, au moins pour l'approvisionnement en silex. La Loire est donc proche, omniprésente, mais ses rivages ne sont peut-être pas des plus confortables. Le cours du fleuve est certainement beaucoup plus encaissé qu'aujourd'hui; la forme en tresse qui s'étend largement dans la vallée cède le pas à un chenal moins large et plus profond. La mer entame une brusque remontée vers 14000 cal BP, soit un peu avant l'Alleröd. Pendant cet interstade, le niveau marin passe d'environ $85 \mathrm{~m}$ à $65 \mathrm{~m}$ sous le niveau actuel, si l'on en croit les courbes obtenues sur les récifs coralliens du Pacifique (Pirazzoli, 1991; Bard et al., 1996). Les effets de cette transgression sur la Loire et l'Aubance ne se font pas encore sentir. En revanche, il sera peut-être un jour possible de prendre en compte la restriction des territoires de chasse que cette transgression suppose, les déplacements de populations humaines et les changements économiques induits.

La cuvette des Chaloignes associe un réseau hydrographique dense et une protection relative contre les vents. Elle est le siège d'une source aujourd'hui pérenne dans le 
vallon ouest, mais on ne peut que supposer son existence au Tardiglaciaire. Abritée des vents, ses versants fixés par une couverture végétale arborée plus abondante, la discrète cuvette des Chaloignes a pu sembler plus accueillante que le bord du fleuve. Les hommes se sont alors installés sur les versants du vallon occidental, autour de l'actuelle source, et les zones périphériques furent moins densément occupées. Même si le jeu des occupations entre le centre et la périphérie de la cuvette reste difficile à appréhender, on observe globalement des occupations de même type, sans structure massive et avec un large éventail d'outils. Mais cette monotonie apparente se fissure si l'on examine le déroulement des chaînes opératoires de débitage au sein de chaque locus.

\section{LA SEGMENTATION DES CHAÎNES OPÉRATOIRES COMME SECOND INDICE ÉCONOMIQUE}

Si le travail du technologue est la reconstitution du débitage dans son déroulé, les interruptions dans ce processus vont intéresser davantage l'économiste, qui y verra les effets d'un déplacement au sein du site ou hors du site. Sous quelle forme le silex est-il arrivé? Quels outils manquent sur un locus? L'homogénéité générale de l'industrie azilienne aux Chaloignes indique une même phase chronologique, dont on serait bien en peine de chiffrer la durée. Les hommes sont revenus plusieurs fois dans ces vallons, car certaines matières importées ne sont présentes qu'à un endroit et cette absence de contact entre locus plaide pour un fractionnement des occupations dans le temps. D'ailleurs, les remontages entre locus sont presque inexistants. Par ailleurs, la masse de silex impliquée et l'étendue du site nous convainquent d'apports réguliers.

Les contacts ou les déplacements directs mettent les hommes en relation avec des groupes de l'ouest et de l'est, à plusieurs dizaines de kilomètres, au moins dans l'axe de la Loire et évidemment peut-être ailleurs. L'ocre est récupérée par-delà la Loire, à une dizaine de kilomètres au nord. Si les galets de silex sont très souvent travaillés dès le début sur les locus, les autres roches (quartzites de Montbert, silex en plaquette $\mathrm{M}$, etc.) arrivent largement débitées. Pourquoi sont-elles parfois représentées seulement par un ou deux éclats? Le processus de débitage est soit très fractionné sur le territoire de nomadisme (on tire quelques éclats d'un nucleus pour un besoin particulier), soit scindé dans l'habitat lui-même (hypothèse des locus complémentaires ou simplement contemporains). Mais dans ce cas, nous aurions peut-être davantage de remontages entre zones. C'est donc la première hypothèse vers laquelle nous inclinons.
Les activités réalisées sur chaque locus réclament sensiblement toute la panoplie de l'outillage azilien; c'est l'un des principaux enseignements de ce site. Cela ne signifie pas nécessairement que ce sont les mêmes activités qui ont été à chaque fois réalisées. Sur le locus 1 ont été détectées une fabrication d'armatures, une réfection d'arme comprenant à la fois l'armature de pierre mais aussi le raclage possible d'un fût en bois, une fabrication d'outils communs, un travail des peaux dans une phase terminale. Il conviendrait de prolonger cette enquête tracéologique sur d'autres locus. Les concentrations particulières d'outils décelées à certains endroits témoignent peut-être d'une certaine diversité des actions au fil des migrations et des cycles saisonniers. Un locus a pu accueillir des chasseurs au retour d'une expédition, un autre témoignerait d'un traitement des peaux, un autre encore serait le siège de la préparation d'une partie de pêche, un autre...

Les déchets lithiques se sont accumulés sur des surfaces restreintes et indiquent soit des aires d'activités, soit des zones dépotoirs; nous n'avons jamais été capables de trancher entre ces hypothèses. La superposition d'un locus et d'une habitation (le traditionnel «fond de cabane») est à exclure et les abris forcément légers sont à proximité des tas de silex abandonnés. Les foyers ne sont jamais appareillés, alors que les blocs de quartz ou les blocs de grès résiduels existent dans le proche environnement: est-ce l'argument le plus décisif pour supposer des séjours courts? Bâtons d'ocre et galets gravés témoignent de croyances aujourd'hui disparues, seuls indices matériels qui rappellent au Préhistorien qu'il doit freiner sa lecture trop économique ou trop déterministe, pour envisager un espace de vie évidemment chargé de symboles, structuré en définitive par une vision du monde toute particulière, que l'archéologie ne saurait pénétrer.

\section{Remerciements}

La liste des intervenants est tellement longue et la place tellement restreinte qu'il nous faut globaliser nos remerciements, notre gratitude ne s'en trouvant pas diminuée. Nous remercions donc avec la plus grande vigueur les multiples fouilleurs de cette opération, les membres de la coordination AFAN de l'A87, du Service régional de l'Archéologie, de la Société Nantaise de Préhistoire et du département géologie de l'Université d'Angers. Des chercheurs de talent ont bien voulu nous appuyer ou corriger nos erreurs; nous tenons à les remercier, sans pour autant leur faire endosser nos éventuelles divagations: P. Bodu, J.-P. Bouvet, J.-M. Gouédo, N. Le Meur, D. Poncet, A. Tresset et C. Verjux.

G. M. 


\section{BIBLIOGRAPHIE}

\begin{tabular}{ll}
\multicolumn{1}{c}{ ABRÉVIATIONS } \\
AFEAF & Association française pour l'étude de l'âge du Fer. \\
AFEQ & Association française pour l'étude du Quaternaire \\
AMARAI & Association Manche Atlantique pour la recherche archéologique dans les îles. \\
APRAIF & Association pour la recherche archéologique en Île-de-France. \\
BAR & British Archaeological Report. \\
BSPF & Bulletin de la Société préhistorique française. \\
CTHS & Comité des travaux historiques et scientifiques. \\
DAF & Documents d'archéologie française. \\
ERAUL & Études et recherches de l'université de Liège. \\
INA-PG & Institut national agronomique - Paris-Grignon \\
MSH & Maison des sciences de l'homme. \\
RAO & Revue archéologique de l'Ouest. \\
SPF & Société préhistorique française. \\
UISPP & Union internationale des sciences préhistoriques et protohistoriques.
\end{tabular}

\section{ALLARD M.}

1982: «Un habitat de chasseurs au camp d'Auvours", in COLLECTIF (DIR.), Les Habitats du Paléolithique supérieur, Actes du colloque international de Roanne-Villerest, 22-24 juin 1982, Roanne-Villerest, p. $77-79$.

1983: «État de la question sur le Paléolithique supérieur en Mayenne: les grottes de Thorigné-en-Charnie et de Saint-Pierresur-Erve », $B S P F, 80$, p. 10-12.

\section{Allard M., Gruet M.}

1976: «Les civilisations du Paléolithique supérieur dans les Pays de la Loire», in LUMLEY DE H. (DIR.), La Préhistoire française -I-2- Civilisations paléolithiques et mésolithiques, Paris, éd. du CNRS, p. 1305-1310.

\section{ALLARD M., GUYOT R.}

1972: «Étude préliminaire d'une industrie magdalénienne au Camp d'Auvours, commune de Saint-Mars-la-Brière (Sarthe) », BSPF, 69, 1, p. 347-355.

AVERY B. W.

1985: "Argillic Horizons and their Significance in England and Wales ", in BOARDMAN J. (DIR.), Soils and Quaternary Landscape Evolution, Chichester, A Wiley International Publications, p. 69-86.

\section{BARBIER D.}

1999: Histoire de la végétation du Nord-Mayennais de la fin du Weichsélien à l'aube du XXI e siècle: mise en évidence d'un Tardiglaciaire armoricain, Interactions Homme-Milieu, Nantes, Gemina, 2 vol
BARBIER D., VISSET L.

2000: «Les spécificités d'un Tardiglaciaire armoricain : étude pollinique synthétique à partir de trois tourbières du nord-est mayennais (France)", Quaternaire, 11, 2, p. $99-106$.

BARd E., HAMElin B., ARNold M., MONTAGgioni M., CABIOCH G., FAURE G., ROUGERIE F.

1996: «Deglacial Sea-Level Record from Tahiti Coral and the Timing of Global Meltwater Discharge», Nature, 382, 18 juillet 1996, p. 241-244.

\section{BARTON R. N. E., ROBERTS A. J.}

1997: «Systèmes économiques et modalités techniques dans l'ouest de la GrandeBretagne au Tardiglaciaire», in FAgnart J.-P., ThÉVENIN A. (DIR.), Le Tardiglaciaire en Europe du Nord-Ouest, Actes du CXIX $X^{e}$ congrès national des Sociétés historiques et scientifiques, Amiens, 26-30 oct. 1994, Paris, éd. du CTHS, p. 507-516.

Bazin P., Gallais J.-Y., Mornand J., THÉVENIN A.

1995: Schistes gravés sur le cours inférieur de la Loire, Besançon (coll. Annales littéraires de l'université de Besançon, 567), p. 107-117.

\section{BÉMILLI C.}

2000: «Nouvelles données sur les faunes aziliennes du Closeau, Rueil-Malmaison (Hauts-de-Seine) », in PION G. (DIR.), Le Paléolithique supérieur récent: nouvelles données sur le peuplement et l'environnement, Actes de la table ronde de Chambéry,
Paris, éd. SPF (coll. Mémoire de la SPF, XXVIII), p. 29-38.

\section{BIGNON O., BODU P.}

2006: "Stratégie cynégétique et mode de vie à l'Azilien ancien dans le Bassin parisien: les apports de l'exploitation des chevaux du Closeau (niveau inférieur; Rueil-Malmaison, Hauts-de-Seine) », L'Anthropologie, 110, p. 401-417.

\section{BODU P.}

1995: «Un gisement à Ferdermesser sur les bords de la Seine: le "Closeau" à RueilMalmaison (Hauts-de-Seine) », BSPF, 92, p. $451-456$.

2000a: «Les faciès tardiglaciaires à grandes lames rectilignes et les ensembles à pointes de Malaurie dans le sud du Bassin parisien: quelques réflexions à partir de l'exemple du gisement du Closeau (Hauts-de-Seine)», in CROTTI P. (DIR.), Meso'97, Table ronde sur l'Épipaléolithique et le Mésolithique, Lausanne, 21-23 nov. 1997, Lausanne, Cahiers d'archéologie romande (coll. Cahiers d'archéologie romande, 81 ), p. 9-28.

2000b: «Que sont devenus les Magdaléniens du Bassin parisien?: quelques éléments de réponse sur le gisement azilien du Closeau (Rueil-Malmaison, France) ", in VALENTIN B., BODU P., Christensen M. (DIR.), L'Europe centrale et septentrionale au Tardiglaciaire, Actes de la table ronde internationale de Nemours, 14-16 mai 1997, Nemours, éd. APRAIF, p. 315-339. 
BODU P., BÉMILli C.

2000: «Le gisement du Closeau à RueilMalmaison (Hauts-de-Seine): le lion est-il mort ce soir?", in CUPILlaRd C., RICHARD A. (DIR.), Les Derniers chasseurscueilleurs d'Europe occidentale (13000-5500 avant J.-C.), Actes du colloque international de Besançon, 23-25 oct. 1998, Besançon, Presses universitaires franc-comtoises, p. 173-186.

Bodu P., Julien M., Valentin B., DEBOUT G.

2006: «Un dernier hiver à Pincevent: les Magdaléniens du niveau IV 0 (Pincevent, La Grande-Paroisse, Seine-et-Marne) », Gallia Préhistoire, 48, p. 1-180.

\section{Bodu P., VALENTIN B.}

1997: «Groupes à Federmesser ou aziliens dans le sud et l'ouest du Bassin parisien: propositions pour un modèle d'évolution », BSPF, 94, 3, p. 341-347.

BOND G., BROECKER W., JOHNSEN S., MCMANUS J., LABEYRIE L., JOUZEL J., BONANI G.

1993: «Correlations between Climate Records from North Atlantic Sediments and Greenland Ice», Nature, 365, p. 143-147.

Braguier A., Cadot R., Mornand J.

1988: "Nouvelles pierres gravées en Maineet-Loire», Bulletin trimestriel de la Société d'études scientifiques de l'Anjou, 73, p. 26-27.

\section{BRIDAULT A.}

1997: «Chasseurs, ressources animales et milieux dans le nord de la France, de la fin du Paléolithique à la fin du Mésolithique: problématique et état de la recherche", in FAgnart J.-P., ThÉVENIN A. (DIR.), Le Tardiglaciaire en Europe du Nord-Ouest, Actes du CXIX congrès national des Sociétés historiques et scientifiques, Amiens, 26-30 oct. 1994, Paris, éd. du CTHS, p. 165-176.

\section{BULLOCK P.}

1985: "The Role of Micromorphology in the Study of Quaternary Soil Processes ", in BOARDMAN J. (DIR.), Soils and Quaternary Landscape Evolution, Chichester, A Wiley International Publications, p. 45-68.

CASPAR J.-P., BIE M. DE.

1996: «Preparing for the Hunt in the Late Paleolithic Camp at Rekem, Belgium", Journal of Field Archaeology, 23, 4, p. 437-460.

2000: Rekem: a Federmesser Camp on the Meuse River Bank, Leuven, Leuven University
Press; Asse-Zellik, Instituut voor het Archeologisch Patrimonium, 2 vol., 325 p. et 265 p.

GaVet P., ARnaud A., Blaise J., Brossé R., ChaURis L., Gruet M., LARDEUX H.

1970: Orléans, Feuille Angers XV - 22 à 1/50000, Bureau de recherche géologiques et minières.

\section{CÉLÉRIER G.}

1998: «L'abri sous-roche de Pont d'Ambon à Bourdeilles (Dordogne, France) : perspective synthétique", Paléo, 10, p. 233-264.

\section{CÉLÉRIER G. (DIR.)}

1994: «L'abri-sous-roche de Pont-d'Ambon à Bourdeilles (Dordogne)», Gallia Préhistoire, 36, p. 65-144.

\section{Célérier G., Chollet A., Hantaï A}

1997: «Nouvelles observations sur l'évolution de l'Azilien dans les gisements de Bois-Ragot (Vienne) et de Pont-d'Ambon (Dordogne) », BSPF, 94, 3, p. 331-336

\section{CÉLÉRIER G., MOSS E.}

1983: "L'abri sous-roche de Pont d'Ambon à Bourdeilles (Dordogne), un gisement magdalénien-azilien: micro-traces et analyse fonctionnelle de l'industrie lithique», Gallia Préhistoire, 26, 1, p. $81-108$.

Célérier G., Nisole S., Beaune S. DE 1993: «L'abri-sous-roche de Pont-d'Ambon à Bourdeilles (Dordogne) ", Gallia Préhistoire, 35, p. 1-137.

Chappellaz J., Blunier T., Raynaud D., BARNOLA J.-M., SCHWANDER J. STAUFFER B.

1993: «Synchronous Changes in Atmospheric CH4 and Greenland Climate between 40 and 8 Kyr B.P. », Nature, 366, p. 443-445.

\section{CHEVAlieR G.}

1999: «De nouveaux indices du Paléolithique supérieur sur le littoral trégorrois (Côtesd'Armor) ", AMARAI, 12, p. 29-49.

\section{Chollet A.}

1990: «La grotte du Bois-Ragot à Gouex (Vienne)", in JOUSSAUME R., Pautreau J.-P. (DIR.), La Préhistoire du Poitou, Rennes, éd. Ouest-France (coll. Ouest-France Université), $600 \mathrm{p}$.

Chollet A., DUJARdin V. (DIR.)

2005: La Grotte du Bois-Ragot à Gouex (Vienne), Magdalénien et Azilien: essais sur les hommes et leur environnement, Paris, éd. SPF (coll. Mémoire de la SPF, 38), 427 p.

\section{COLLECTIF}

1984: «Les sites à microlithes entre Vilaine et Marais poitevin", Études préhistoriques et protohistoriques des Pays de la Loire, 7, $300 \mathrm{p}$.

COUdRet P., FAGNART J.-P.

1997: «Les industries à Federmesser dans le Bassin de la Somme: chronologie et identité des groupes culturels ", BSPF, 94, 3, p. 349-359.

COUdRet P., LARRIÈre-CABIRAN M. Olive M., Pigeot N., Taborin Y.

1994: «Étiolles», in TABORIN Y. (DIR.) Environnements et habitats magdaléniens dans le centre du Bassin parisien, Paris, éd. de la MSH (coll. DAF, 43), p. 132-146.

\section{COULONGES L.}

1963: «Magdalénien et Périgordien postglaciaire: la grotte de la Borie-del-Rey (Lot-et-Garonne) ", Gallia Préhistoire, VI, p. 1-29.

\section{Couraud C.}

1985: L'Art azilien: origine-survivance, Paris, CNRS Éditions (coll. Suppl. à Gallia Préhistoire, XX), $176 \mathrm{p}$.

\section{CRemaschi M.}

1990: «Depositional and Post-Depositional Processes in Rock Shelters of Northern Italy during the Late Pleistocene: their Paleoclimatic and Paleoenvironnemental significance », Quaternaire, 1, p. 51-64.

\section{CURMi P.}

1987: «Sur la signification des revêtements complexes argileux et limoneux dans les sols lessivés acides", in Micromorphologie des sols/Soil Micromorphology, Plaisir, Association française pour l'étude des sols, p. 251-255.

DAVID F., ORLIAC M.

1994: "Pincevent», in TABORIN Y. (DIR.), Environnements et habitats magdaléniens dans le centre du Bassin parisien, Paris, éd. de la MSH (coll. DAF, 43), p. 154-166.

\section{D'ERRICO F}

1994: L'Art gravé azilien: de la technique à la signification, Paris, CNRS Éditions (coll. Suppl. à Gallia Préhistoire, XXXI), 329 p.

DRUCKER D., GÉLÉRIER G.

2001: "Teneurs en carbone-13 du collagène de grands mammifères du site de Pont 
d'Ambon (Dordogne, France) : implications pour l'environnement et son exploitation au Tardiglaciaire dans le sud-ouest de la France», Paléo, 13, p. 145-158.

FAGNART J.-P.

1997: La Fin des temps glaciaires dans le nord de la France: approche archéologique et environnementale des occupations humaines $d u$ Tardiglaciaire, Paris, éd. SPF (coll. Mémoire de la SPF, XXIV), $270 \mathrm{p}$.

FAGNART J.-P., COUdRET P.

2000: «Données récentes sur le Tardiglaciaire $\mathrm{du}$ bassin de la Somme», in PION G. (DIR.), Le Paléolithique supérieur récent: nouvelles données sur le peuplement et l'environnement, Actes de la table ronde de Chambéry, Paris, éd. SPF (coll. Mémoire de la SPF, XXVIII), p. 113-126.

FEdoroff N., Courty M.-A.

1994 (rééd.): "Organisation du sol aux échelles microscopiques ", in BONNEAU M, SOUChitr B. (DIR.), Pédologie, vol. 2, Paris, Masson.

FEDOROFF N., GOLDberG P.

1982: «Comparative Micromorphology of Two Late Pleistocene Paleosols (in the Paris Bassin)", Catena, 9 (3/4), p. 227-232.

Fosse G., VAlentin B., Billard C.

1997: «Une occupation des groupes à Federmesser en Haute-Normandie: le gisement du Cornet à Ambenay (Eure) », in FAGNART J.-P., ThÉVENIN A. (DIR.), Le Tardiglaciaire en Europe du Nord-Ouest, Actes du CXIX $X^{e}$ congrès national des Sociétés historiques et scientifiques, Amiens, 26-30 oct. 1994, Paris, éd. du CTHS, p. 245-255.

FOUChER P., SAN JUAN C.

1994: «Le Tardiglaciaire sur le littoral charentais : état de la question et nouvelles perspectives ", in GONZALES MORALES G. A., Clark M. (DIR.), Old People and the Sea, International Conference on the Mesolithic of the Atlantic Façade, Santander, 6-9 juill. 1994, Pré-Actes, p. 3.1-3.8.

\section{FOUÉRÉ $\mathbf{P}$.}

2005: "Les matières premières siliceuses du site du Bois-Ragot, Gouex (Vienne)", in Chollet A., Dujardin V. (DIR.), La Grotte du Bois-Ragot à Gouex (Vienne), Magdalénien et Azilien: essais sur les hommes et leur environnement, Paris, éd. SPF (coll. Mémoire de la SPF, XXXVIII), p. 29-42.
Gagnepain J., Bracco J.-P., Bertran P., BEZJ.-F., Bibart P., CaNAls I SAlOMOA., Jorda C., Jorda M., Philibert S., STOUVENOT C., VIGIER S. (DIR.)

1999: «Saint-Antoine à Vitrolles, locus 2 (Hautes-Alpes): premiers résultats des fouilles de sauvetage urgent (1995-1996) d'un gisement épigravettien ", BSPF, 96, 2, p. 191-202.

\section{GALLAIS J.Y.}

1984: «Stations à microlithes de Clis à Guérande (Loire-Atlantique)», Études préhistoriques et protohistoriques des Pays de Loire, 7, p. 31-43.

GARROD D. A. E.

1926: The Upper Paleolithic Age in Britain, Oxford, Clarendon Press.

GÉ T., Courty M.-A., Wendy M., WATTEZ J.

1993: "Sedimentary Formation Processes of Occupation Surfaces", in GOLDBERG P., Petraglia M. (DIR.), Formation Processes in Context, Madison, Wisconsin, Prehistory Press, p. 149-162.

\section{GIOT P.-R., HALlEGOUET B.} MONNIER J.-L.

1977: «Le Paléolithique supérieur du Pays de Léon (Finistère): les gisements de Roc'h Toul, Parc ar Plenen (Guiclan), Enez Guennoc (Landéda) et la ForestLanderneau ", L'Anthropologie, 81, 2, p. 201-234.

\section{GonZalez Urouijo J. E., Ibanez Esteve} $\mathrm{Z} \mathrm{J} \cdot \mathrm{J} \cdot$

1993: "Utilizacion del instrumental litico y functionalidad del asentamiento en el yacimiento de Berniollo (Alava, Espana) », in ANDERSON-GERFAUD P., BEYRIES S., OTTE M., Plisson H. (DIR.), Traces et fonctions: les gestes retrouvés, Actes du colloque international de Liège, 8-10 déc. 1990, Liège (coll. ERAUL, 50), 1, p. 97-104.

GOULETQUER P., KAYSER O., LE GOFFIC M., LEOPOLD P., MARGHAND G., MOULLEC J.-M.

1996: «Où sont passés les Mésolithiques côtiers bretons: bilan 1985-1995 des prospections de surface dans le Finistère", $R A O, 13$, p. 5-30.

\section{GOULETOUER P., LEOPOLD P.}

1991: "Le site mésolithique de Quillien (Le Cloître-Saint-Thégonnec, Finistère) ", $R A O, 8$, p. 61-82.

\section{Gouletouer P., MonNier J.-L.}

1976: «Les civilisations de l'Épipaléolithique et du Mésolithique en Armorique", in Lumley H. DE, GUilaine J. (DIR.), La Préhistoire française, 1, vol. 2.

Gouraud G., JAUNEAU J.-M., GANDRIAU O., DUGAST C.

1998: «Les instruments tardiglaciaires de Coex/L'Aiguillon-sur-Vie (Vendée)», Groupe vendéen d'études préhistoriques, 34, p. $4-15$.

\section{Gouraud G., LE GofFIC M.}

1998: «L'importance du gîte montbertain sur l'approvisionnement et la diffusion de la roche clivable dans la Préhistoire régionale", Société nantaise de Préhistoire, 21, p. 20-27.

\section{GRIGGO C.}

2005: «Les grands mammifères de la grotte du Bois-Ragot», in CHOLLET A., DUJARDIn V. (DIR.), La Grotte du BoisRagot à Gouex (Vienne), Magdalénien et Azilien: essais sur les hommes et leur environnement, Paris, éd. SPF (coll. Mémoire de la SPF, XXXVIII), p. 289-318.

\section{GRUeT M.}

1938: «Prise de date sur la découverte du site d'Écouflant», BSPF, XXXV, 1, p. 86-87.

1943: «Une station mésolithique à Écouflant (Maine-et-Loire) ", BSPF, LXVIII-LXXIII (1938-1943), p. 37-43.

1973: «Notice sur les environs d'Angers", Annales scientifiques de l'université de Besançon, Géologie, $3^{\text {e }}$ série, fasc. 21, p. 63-65.

1990: Roc-en-Pail: 50000 ans de Préhistoire angevine, Catalogue d'exposition, Angers, 1990, $36 \mathrm{p}$.

\section{Gruet M., JaOUen P.}

1957: «Bégrolles et la pénétration magdalénienne en Loire-Inférieure », BSPF, LIV, 7-8, p. 341-452.

\section{HUIJZER A. S.}

1993: Cryogenic Microfabrics and Macrostructures: Interrelations, Processes, andPaleoenvironment Significance, Netherlands Academisch Proefschrift, Vrije Universiteit.

JAUNEAU J.-M., Gouraud G., DUGaST C. 2000: «Premiers éléments tardiglaciaires en Vendée ", $R A O, 17$, p. 25-35.

Joussaume R., Pautreau J.-P.

1990: La Préhistoire du Poitou, Rennes, éd. Ouest-France, $600 \mathrm{p}$. 
JUDE P.-E.

1960: La Grotte de Rochereille, station magdaléenienne et azilienne, Paris, Masson (coll. Archives de l'Institut de paléontologie humaine, mémoire, 30), $75 \mathrm{p}$.

\section{KAYSER O., BERNIER G.}

1988: «Nouveaux objets décorés du Mésolithique armoricain", BSPF, 85, p. 45-47.

\section{LAPLACE-JAURETCHE G.}

1957: «Les industries de Roc'h Toul et Parar-Plenen en Guiclan (Finistère) ", BSPF, LIV, 7-8, p. 422-438.

laporte l., Marchand G., Sellami F., OBERLIN C., BRIDAUlT A.

2000: «Les occupations mésolithiques et du Néolithique ancien sur le site de la Grange à Surgères (Charente-Maritime) ", RAO, 17, p. 101-142.

\section{LE GOFFIC M.}

1993: «Les pierres gravées de Goarem Kerbilaeten Plonéour-Lanvern (Finistère) et leur contexte méso-néolithique ", $R A O$, 10, p. 19-31.

1996: «Sibiril, Le Carpont» in «Notice d'archéologie finistérienne (1995)», Bulletin de la Société archéologique du Finistère, CXXV, p. 73.

2001: «Trémaouézan, Lann-Gazel», in "Notice d'archéologie finistérienne (2001) ", Bulletin de la Société archéologique du Finistère, CXXX, p. 98-100.

\section{LEROI-GOURHAN A., BRÉZILLON M.}

1972: «Fouilles de Pincevent: essai d'analyse ethnographique d'un habitat magdalénien (la section 36)", Paris, CNRS Éditions (coll. Suppl. à Gallia Préhistoire, VII), 2 vol., 327 p. et plans.

\section{LE TENSORER J.-M.}

1981: Le Paléolithique de l'Agenais, Paris, éd. du CNRS (coll. Cahiers du Quaternaire, 3), $526 \mathrm{p}$.

\section{LEVILLAYER A.}

2006: «L'occupation de l'âge du Fer aux Chaloignes (Mozé-sur-Louet, Maine-etLoire) », RAO, 23, p. 117-135.

\section{LOZET J., MATHIEU C.}

1990: Dictionnaire de Science du sol, Paris, Lavoisier (coll. Technique et documentation).

\section{MAGNY M.}

1995a: «Successive Oceanic and Solar Forcing Indicated by Younger Dryas and Early Holocene Climatic Oscillations in the Jura", Quaternary Research, 43, p. $279-285$.

1995b: «Les sédiments des lacs: miroir du climat », La Recherche, 282, p. 60-65.

\section{MARCHAND G.}

1991: Le Mésolithique de la Tourasse (SaintMartory, Haute-Garonne): première approche typologique et technologique des industries lithiques (fouilles Michel Orliac), Mémoire de DEA, Univ. Paris-I.

2005: «Le Mésolithique final en Bretagne : une combinaison des faits archéologiques », in MARChand G., TRESSET A. (DIR.), Unité et diversité des processus de néolithisation sur la façade atlantique de l'Europe (7-4 millénaires avant J-C.), Paris, éd. SPF (coll. Mémoire de la SPF, XXXVI), p. 67-86.

2008: «Dynamique des changements techniques sur les marges du Massif armoricain de l'Azilien au Premier Mésolithique ", in FAGNARTJ.-P., THÉVENINA., DUCROCQT., SOUFFI B, COUDRET P. (DIR.), Le Début du Mésolithique en Europe du Nord-Ouest, Actes de la table ronde d'Amiens, 9-10 oct. 2004, Paris, éd. SPF (coll. Mémoire de la SPF, $\mathrm{XLV})$, p. 52-64.

MARGhand G., ARThUis R., AUXietTe G., FORRÉ P., LETHO DUCLOS Y., Rimbault S., SEllami F., SiCARd S.

2000: Les Chaloignes, Mozé-sur-Louet, Série Fouilles, (coll. Document final de synthèse de sauvetage urgent, A 87), 2 vol., 260 p. et $120 \mathrm{p}$.

MARCHANDG.,BLANCHETS., CHEVAliERG., Gallais J.-Y., LE GOFFIC M., NAUDINOT N., YVEN E.

2004: «La fin du Tardiglaciaire sur le Massif armoricain: territoires et cultures matérielles", Paléo, 16, p. 137-170.

MARCHAND G., LAPORTE L., BRIDAUlt A., Giraud C., Giraud T., Sellami F.

2000: «L'habitat mésolithique et néolithique de la Grange, à Surgères (CharenteMaritime)", in CUPILlARD C., RICHARD A. (DIR.), Les Derniers chasseurscueilleurs d'Europe occidentale (13000-5500 avant J.-C.), Actes du colloque international de Besançon, 23-25 oct. 1998, Besançon, Presses universitaires franc-comtoises, p. $253-264$

MARSHACK A.

1972: Les Racines de la civilisation: les sources cognitives de l'art, du symbole et de la notation chez les premiers hommes, Paris, Plon, $415 \mathrm{p}$.

\section{MAURY J.}

1995: «Une pierre plantée sauveterrienne dans l'abri $\mathrm{n}^{\circ} 2$ des Usclades (Nant, Aveyron)", BSPF, 92, 4, p. 457-458.

\section{MONNIER J.-L.}

1980: Le Paléolithique de la Bretagne dans son cadre géologique, Rennes, Travaux du laboratoire d'anthropologie.

\section{MORNAND J.}

1982: «Les schistes gravés de La Pommeraye (Maine-et-Loire) », Bulletin trimestriel de la Société d'études scientifiques de l'Anjou, N. S., 11, p. 29-45.

1987: «Nouvelles pierres gravées à La Pommeraye (Maine-et-Loire)», Bulletin trimestriel de la Société d'études scientifiques de l'Anjou, 70, p. 19-22.

1990: «Note sur la découverte de deux nouvelles ardoises gravées à La Pommeraye (Maine-et-Loire)», Bulletin trimestriel de la Société d'études scientifiques de l'Anjou, 78, p. 12-18.

1992: «Un site de campement épipaléolithique à La Pommeraye (Maine-et-Loire) », Bulletin de la Société d'études scientifiques de l'Anjou, 14, p. 49-56.

\section{MOUYOUNGOU J.}

1990: Les Silicifications mésozoïques et cénozoïques de la bordure occidentale du bassin de Paris et de la région de Brazzaville au Congo, Thèse de doctorat, Univ. d'Angers, $250 \mathrm{p}$.

\section{MUSCH J.}

1997: L'Étang du Cruchet, commune d'Écommoy (72), Rapport de fouille A28, série fouille, $\mathrm{n}^{\circ} 3$.

\section{ORDAZ CHAPARRO V.}

1995: Impact de la culture intensive des agrumes sur le fonctionnement des sols dans une zone tropicale du Mexique (état de Tabasco), Thèse de doctorat, INA-PG

\section{ORLIAC E., ORLIAC M}

1973: "La succession des industries dans la grotte de la Tourasse (Saint-Martory, Haute-Garonne) », $B S P F, 70$, p. 66-68

ORLIAC M.

1975: «La grotte de la Tourasse», Bulletin de l'AFEQ, 3-4, p. 189-190. 
PASTy J.-F., Alix P., Ballut C., Griggo C., MURAT R.

2002: «Le gisement épipaléolithique à pointes de Malaurie (Les Martres-d'Artière, Puyde-Dôme) ", Paléo, 14, p. 101-176.

Péquart M., PéQuart S.-J., Boule M., VALLOIS H.

1937: Téviec, station nécropole mésolithique du Morbihan, Paris, Masson (coll. Mémoire des archives de l'Institut de paléontologie humaine, 18).

\section{PERPÈRE M.}

1976: «Les civilisations de l'Épipaléolithique et du Mésolithique en Poitou-Charentes ", in Guilaine J. (DIR.), La Préhistoire française, II, Les Civilisations néolithiques et protohistoriques de la France, p. 1436-1440.

\section{PHILIBERT S.}

1991: «Fontfaurès: analyse tracéologique de l'industrie lithique et approche fonctionnelle du site", in BARBAZA M. ET AL. (DIR.), Fontfaurès en Quercy: contribution à l'étude du Sauveterrien, Toulouse, EHESS (coll. Archives d'écologie préhistorique, 11), p. 151-169.

1995: «Les grottes Jean-Pierre 1 et 2 à SaintThibaud-de-Couz (Savoie) : analyse fonctionnelle des outillages de pierre", in BINTZ P. (DIR.), «Les grottes Jean-Pierre 1 et 2 à Saint-Thibaud-de-Couz (Savoie) : paléo-environnement et cultures du Tardiglaciaire à l'Holocène dans les Alpes du Nord», Gallia Préhistoire, 37, p. 287-316.

2002: Les Derniers «Sauvages»: territoires économiques et systèmes techno-fonctionnels mésolithiques, Oxford, Archaeopress (coll. BAR International Series, 1069), p. 193.

\section{Pigeot N.}

1987: Magdaléniens d'Étiolles: économie de débitage et organisation sociale, Paris, CNRS Éditions (coll. Suppl. à Gallia Préhistoire, XXV), $168 \mathrm{p}$.

\section{Pirazzoli P. A.}

1991: World Atlas of Holocene Sea-Level Changes, Amsterdam, Londres, New-York, Elsevier (coll. Elsevier Oceanography Series, 58), $300 \mathrm{p}$.

PLISSON H

1985: Étude fonctionnelle d'outillages lithiques préhistoriques par l'analyse des micro-usures: recherche méthodologique et archéologi$q u e$, Thèse de doctorat, Univ. Paris-IPanthéon-Sorbonne.
Puisségur J.-J., Limondin N., ROUSSEAU D.-D.

1994: «Changements environnementaux et climatiques en Bourgogne à l'Holocène à partir de l'étude des mollusques continentaux», Quaternaire, 5, 3-4, p. 189-190.

RozoY J.-G.

1978: «Les derniers chasseurs, l'Épipaléolithique en France et en Belgique », Bulletin de la Société archéologique champenoise, $\mathrm{n}^{\circ}$ spécial juin 1978, $3 \mathrm{t}$.

1997: «Territoires sociaux et environnements en France du Nord et en Belgique de 14000 à $6000 \mathrm{BP}$ », in FAGNART J.-P., THÉVENIN A. (DIR.), Le Tardiglaciaire en Europe du Nord-Ouest, Actes du CXIX ${ }^{e}$ congrès national des Sociétés historiques et scientifiques, Amiens, 26-30 oct. 1994, Paris, éd. du CTHS, p. 429-454.

\section{SCHMIDER B.}

1971: Les Industries lithiques du Paléolithique supérieur en Île-de-France, Paris, CNRS Éditions, (coll. Suppl. à Gallia Préhistoire, IV), $218 \mathrm{p}$

\section{SCHWARTZ D.}

1990: «Relations reliefs-variations paléoclimatiques en Afrique centrale", in SCHWARTZ D., LANFRANCHI R. (DIR.), Paysages quaternaires de l'Afrique centrale atlantique, Bondy, éd. de l'ORSTOM, p. 186-192.

\section{SÉARA F.}

1998: "Habitats de plein air», in CUPILlaRd C., RICHARD A. (DIR.), Les Derniers chasseurs-cueilleurs du Massif jurassien et de ses marges (13000-5500 avant J.-C.), Lons-le-Saunier, Centre jurassien du Patrimoine, p. 170-179.

2000: «Approche de l'organisation spatiale de campements de chasseurs-cueilleurs mésolithiques: le cas de Ruffey-sur-Seille dans le Jura (France)", in CROTTI P. (DIR.), Méso'97, Épipaléolithique et Mésolithique, Actes de la table ronde de Lausanne, 21-23 nov. 1997, Lausanne, Cahiers d'archéologie romande (coll. Cahiers d'archéologie romande, 81), p. 139-150.

\section{SÉara F., Rotillon S., Cupillard C.}

2002: Campements mésolithiques en Bresse jurassienne, Choisey et Ruffey-sur-Seille, Paris, éd. de la MSH, (coll. DAF, 92), $338 \mathrm{p}$.

\section{SELLAMI F.}

1999: Reconstitution de la dynamique des sols des terrasses de la Dordogne à Creysse (Bergeracois): impact sur la conservation des sites archéologiques de plein air, Thèse de doctorat, INA-PG, p. 255.

2002: «Dynamique des sols colluviaux et son impact sur les assemblages anthropiques du site de Solutré (Sâone-et-Loire) », in COMBIER J., MONTET-Wight A. (DIR.), Solutré 1968-1998, Paris, éd. SPF (coll. Mémoire de la SPF, XXX), 281 p.

Sellami F., Teyssandier N., TaHa M.

2002: «Dynamique du sol et fossilisation des ensembles archéologiques sur les sites de plein air: données expérimentales sur l'organisation des traits pédo-sédimentaires ", in BOURGUIGNON L., ORTEGA I., FrÈre-SAUTOT M.-C. (DIR.), Préhistoire et approche expérimentale, Montagnac, éd. Monique Mergoil, p. 313-324.

SICARD-MARCHAND S., ROUZEAU M., ROUZEAU M.

2004: "Nouvelle étude technologique du site de la Vigie-Romaine (Le Croisic) », RAO, 21, p. 5-17.

Siraudeau J., Comte F.

1998: «Les travaux et les jours avec le docteur Michel Gruet, archéologue (1912-1998) », Archives d'Anjou, Mélanges d'histoire et d'archéologie angevines, Angers, éd. Association des amis des archives d'Anjou, t. II, p. 5-36.

Smith J. E., Risk M. J., Schwarcz H. P., MC Connaughey T. A.

1997: «Rapid Climate Change in the North Atlantic during the Youger Dryas Recorded by Deep-Sea Corals", Nature, 386, 24, p. 818-820.

\section{THÉVENIN A}

1982: Rochedane, L'Azilien, l'Épipaléolithique de l'Est de la France et les civilisations épipaléolithiques de l'Europe occidentale, Strasbourg, Univ. des Sciences humaines (coll. Mémoire de la Faculté des sciences sociales, Ethnologie, 1).

1983: «Les galets gravés et peints de l'abri de Rochedane (Doubs) et le problème de l'art azilien ", Gallia Préhistoire, 26, 1, p. 139-188.

1997: “L'“Azilien” et les cultures à pointes à dos courbe: esquisses géographique et chronologique », BSPF, 94, 3, p. 393-411.

\section{TOURNEPICHE J.-F.}

1986: «L'occupation épipaléolithique de la grotte du Quéroy, commune de Chazelles (Charente) », in Préhistoire du Poitou-Charentes: problèmes actuels, Actes $d u$ CXI ${ }^{e}$ congrès des Sociétés savantes, Poitiers, 1986, Paris, éd. du CTHS, p. 207-221. 


\section{VALENTIN B.}

1995: Les Groupes humains et leurs traditions au Tardiglaciaire dans le Bassin parisien: apports de la technologie lithique comparée, Thèse de doctorat, Univ. Paris-I, 3 vol.

1999: "Techniques et cultures: les chasseurs-cueilleurs du Tardiglaciaire dans le sud et l'ouest du Bassin parisien ", in THÉVENIN A., BINTZ P. (DIR.), L'Europe des derniers chasseurs, $V^{e}$ colloque international de l'UISPP, sept. 1995, p. 201-212.

2000: «L'usage des percuteurs en pierre tendre pour le débitage des lames: circonstances de quelques innovations au cours du Tardiglaciaire dans le Bassin parisien", in PION G. (DIR.), Le Paléolithique supérieur récent: nouvelles données sur le peuplement et l'environnement, Actes de la table ronde de Chambéry,
Paris, éd. SPF (coll. Mémoire de la SPF, XXVIII), p. 253-260.

\section{VALENTin B., HANTAï A.}

2005: "Transformations de l'industrie lithique pendant l'Azilien: étude des niveaux 3 et 4 du Bois-Ragot», in Chollet A., Dujardin V. (DIR.), La Grotte du Bois-Ragot à Gouex (Vienne), Magdalénien et Azilien: essais sur les hommes et leur environnement, Paris, éd. SPF (coll. Mémoire de la SPF, XXXVIII), p. 89-182.

\section{VAN VLIET-LANOË B.}

1987: Le Rôle de la glace de ségrégation dans les formations superficielles de l'Europe de l'Ouest: processus et héritages, Thèse d'État, Univ. Paris-I, Caen, EDITEC.
1992: Le Niveau à langues de Kesselt, horizon repère de la stratigraphie du Weischsélien supérieur européen: signification paléoenvironnementale et paléoclimatique, Paris, Société géologique de France (coll. Mémoire de la Société géologique de France, 160), p. 35-44.

\section{WAINWRIGHT J.}

1994: «Erosion of Archaeological Sites: Results and Implications of a Site Simulation Model», Geoarchaeology, 9, 3, p. 173-201.

WOILlaRD G. M., MOOK W. G.

1982: «Carbon-14 Dates at Grande Pile: Correlation of Land and Sea Chronologies ", Science, 215, p. 159-161. 